\title{
Nonlinear Optical Effects in Pure and N-Doped Semiconductors
}

\author{
Dissertation \\ zur Erlangung des Doktorgrades \\ der Mathematisch-Naturwissenschaftlichen Fakultäten \\ der Georg-August-Universität zu Göttingen
}

vorgelegt von

Nias Sven Đonlagić

aus Lübeck

Göttingen 2000 
D7

Referent: Prof. Dr. Kurt Schönhammer Korreferent: Prof. Dr. Reiner Kree

Tag der mündlichen Prüfung: 2.11.2000 


\section{Contents}

1 Introduction $\quad 2$

2 Phonon-Induced Relaxation

in Optically Excited Semiconductors 6

2.1 The Hamiltonian . . . . . . . . . . . . . . . . . . . 7

2.2 Kinetic Equations . . . . . . . . . . . . . . . . . . . . . 9

2.3 Higher Order Corrections . . . . . . . . . . . . . . . . . . . . . . . . . . . . . 13

2.4 Green's Function Approach . . . . . . . . . . . . . . . . . . 18

2.5 Linear Response . . . . . . . . . . . . . . . . . . . . . . 25

2.6 Nonlinear Response . . . . . . . . . . . . . . . . 36

3 Nonlinear Optical Dynamics

of Systems with a Fermi-Edge Singularity 45

3.1 The Hamiltonian . . . . . . . . . . . . . . . . . . . . . . 46

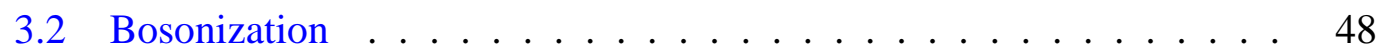

3.3 Time-Dependent Perturbation Theory . . . . . . . . . . . . 52

3.4 Linear Response . . . . . . . . . . . . . . . . . . . . . . . . 54

3.5 Nonlinear Response . . . . . . . . . . . . . . . . . . 61

4 Summary and Outlook $\quad 71$

A Kinetic Equations of Higher Order $\quad 74$

B The Exciton Problem for a Separable Interaction 78

C The Four-Point Response Functions $\quad 81$

C.1 Exact Expressions . . . . . . . . . . . . . . . . . 81

C.2 Approximations ....................... 91

$\begin{array}{ll}\text { D Operator Relations } & 102\end{array}$

$\begin{array}{lll}\text { E Important Functions } & 108\end{array}$ 


\section{Chapter 1}

\section{Introduction}

Over the last decades, the nonlinear optical properties of condensed matter systems have been an attractive and fruitful field of research. While the linear response functions of solids provide information about the elementary excitations of the systems such as excitons and plasmons, nonlinear optical experiments give insight into the dynamics of the fundamental many-body processes which are initiated by the external excitations. Stimulated by the experimental results, new theoretical concepts and methods have been developed in order to relate the observed phenomena to the microscopic properties of the investigated materials. The present work deals with the study of the nonlinear dynamics of pure semiconductors and n-doped semiconductors which are excited by laser pulses whose central frequencies are tuned to the energies of the electronic transitions between the highest valence bands and the lowest conduction bands.

In Chapter 2, the relaxation behavior of optically excited semiconductors under the influence of the interaction between the electrons and longitudinal optical phonons is studied. These studies are mainly motivated by measurements of the differential transmission spectrum in pump-probe experiments [47] and the time-integrated four-wavemixing signals $[39,53]$ in Gallium-Arsenide (GaAs). The pump-probe spectroscopy has especially initiated an intensive theoretical discussion, since it allows the direct observation of the dynamics of the spatial and spectral distribution of the excited carriers. In a typical pump-probe experiment, a sample is excited by two pulses, a strong pump pulse and a weak probe pulse, which propagate in different directions. The interplay between these pulses is then studied by measuring an optical signal which is related to the probe pulses (for example the reflection signal or the transmission signal) as a function of the time delay between both pulses. Depending on the density distributions of the excited conduction electrons and valence holes, the intensity of these signals is either reduced or increased in relation to the signals which are measured in the absence of the pump pulse. In recent years, the development of ultrafast lasers pulses with durations as short as 10 femtoseconds has made it possible to observe the nonlinear dynamics of photoexcited semiconductors in the coherent regime with the help of pump-probe experiments [57]. 
The time behavior of semiconductors which are excited by external electro-magnetic fields is usually described within the framework of the semiclassical Boltzmann equations. The different interaction processes between the electrons and phonons are then described by means of collision terms where the energy is conserved in each collision process. This description, however, can only be valid as long as the dynamics is studied on a time scale which is larger than the characteristic collision times ( 100 - 200 fs) [57]. Consequently, it is insufficient to describe the behavior of the semiconductor with the help of the traditional Boltzmann equations if the dynamics is studied on the femtosecond scale. A better description of the short-time behavior can be achieved by using nonlinear quantum kinetic equations which take quantum phenomena, as for example the energy-time uncertainty, into account. For their derivation, two different theoretical approaches have been employed.

In the first approach, non-equilibrium two-time Green's functions are used as a starting point for the numerical and analytical studies [25, 26, 34, 43]. These functions can be calculated with the help of approximation methods which are similar to the approximation methods for equilibrium Green's functions. The time dependence of the non-equilibrium Green's functions is then determined by the corresponding Dyson equations, which are also denoted as Kadanoff-Baym equations if they are written in a differential form. Since the Green's functions depend on two time variables, it is necessary to introduce an additional approximation method, the so-called Generalized Kadanoff-Baym Ansatz [18], in order to transform the Kadanoff-Baym equations into a system of differential equations for one-time functions. The second approach is based on the Heisenberg equations-of-motion for products of field operators [21, 30, 36, 42, $41,52,56,58]$. As these equations are arranged in an infinite hierarchy, a truncation scheme has to be applied in order to obtain a finite system of differential equations. The truncation is achieved by neglecting correlation functions of higher order. The whole procedure can be viewed as a partial resummation of Feynman diagrams within the framework of the non-equilibrium Green's function approach [37].

The use of the Green's function method has the advantage that it is relatively easy to include certain collective effects such as the screening of the interaction functions or the renormalization of the one-particle propagators. On the other hand, the equationsof-motion method makes it possible to obtain a numerically treatable system of differential equations directly without introducing a complex formalism.

When the quantum kinetic equations for electrons and phonons in a semiconductor are derived with the help of one of the two approaches described above, the electronelectron interaction, which is responsible for the formation of excitons, is often treated in the time-dependent Hartree-Fock approximation, which leads to a renormalization of single particle energies and Rabi frequencies [34, 43, 36]. If, however, the factorization method is applied consequently within the framework of the equations-of-motion method, as it has been done in References [21, 41, 52, 56], additional Coulomb terms appear in the kinetic equations which can be interpreted as vertex corrections to the electron-phonon interaction. Since the effect of these terms on the time behavior of the optically excited semiconductor has not yet been studied in detail, the investiga- 
tions in Chapter 2 will concentrate on the differences between the kinetic equations with and without vertex corrections. For this purpose, the dynamics of the relevant physical quantities, such as the interband polarization or the electron density, will be calculated by using both versions of the kinetic equations.

In Chapter 3, the attention is shifted to the nonlinear behavior of n-doped semiconductors where a non-vanishing density of negative carriers in the conduction band already exists at zero temperature. If the doping is low, it is still possible to observe bound systems of valence holes and conduction electrons in the linear and nonlinear spectrum. In the high-doping regime, however, the picture changes qualitatively. While the bound states vanish because of the presence of the Fermi sea in the conduction band, it is possible to detect an asymmetric algebraic singularity at the Fermi-edge in the absorption spectrum.

The same phenomenon can also be observed in the absorption spectrum of metals and has initiated a considerable theoretical discussion about the different factors which influence the behavior of the absorption spectrum near the threshold. Mahan showed that the occurrence of this singularity can be attributed to the attractive interaction between the conduction electrons and the valence holes which are created by the external light pulses [10]. Detailed analytical calculations by Nozières and his coworkers $[13,14,12,16]$ led to the realization that the power-law divergence at the Fermi-edge can be reduced, or even suppressed, if the reaction of the entire Fermi sea on the appearance of the valence holes is taken into account. It has been shown that the algebraic singularity can be described by means of an exponent $\alpha$ whose value is determined by the scattering phase shift for the conduction electrons in the presence of the attractive potential of the valence holes. A comprehensive presentation of the results can be found in Mahan's textbook on many-body physics [19].

The different physical effects which determine the form of the Fermi-edge singularity can already be understood within the framework of an exactly solvable, onedimensional model where the electrons in the conduction band are described with the help of the Tomonaga-Luttinger Hamiltonian [1, 6]. The elementary excitations of the Fermi sea can then be described in terms of density waves with a linear dispersion. When this auxiliary model was introduced for the first time [15] the restriction to one dimension was justified by the fact that the original three-dimensional problem can be interpreted as an effective one-dimensional problem because the interaction between the valence holes and the conduction electrons is dominated by s-wave scattering processes, if the interaction potential is short-ranged. The investigation of semiconductor quantum wire structures, which show pronounced Fermi-edge singularities in the linear optical absorption spectrum [22], has increased the interest in this model during recent years $[27,29,45,46]$, since it can now be used for the description of real onedimensional systems.

Concerning the theoretical description of the valence band, it has usually been assumed that the valence holes have an infinite mass [13, 14, 12, 16, 15, 19]. Then the positions of the optically created holes remain fixed and the electrons only feel the influence of a time-independent potential. In order to take the effect of a finite hole 
mass on the spectrum into account, a number of approximation methods have been developed since the end of the 1960s [11, 20, 28, 33]. The results indicate that the recoil of the valence holes can lead to a broadening, or even to a disappearance, of the Fermi-edge singularity [11].

During the last decade, the investigation of modulation doped quantum wells using pump-probe spectroscopy [44] has raised interest in the properties of the nonlinear optical response of systems involving a Fermi sea of conduction electrons whose linear absorption spectrum exhibits a threshold singularity. The first theoretical descriptions of these experiments $[35,44,59]$ were based on an approximation technique which was already used successfully for the calculation of the linear response functions [23, 24, 33]. Within the framework of this approximation, the excitations of the Fermi sea in the presence of the valence holes are described with the help of a noncanonical transformation.

Motivated by these developments, the Tomonaga-Luttinger electron model is employed in Chapter 3 in order to describe the general principles of the nonlinear optical response of systems with a Fermi-edge singularity in the linear absorption spectrum using a four-wave-mixing experiment as an example. Within the framework of a standard four-wave-mixing experiment, the sample is excited by three or two pulses with different propagation directions. The interaction between these excitation pulses in the sample leads to the generation of diffracted signals whose intensity can be measured as a function of the time delay between the ingoing pulses. As long as the delay times do not exceed the life-time of the optical excitations, it is possible to observe the coherent time evolution of the excited many-body states under the influence of the interparticle scattering processes. In order to be able to solve the model exactly, the calculations are based on the assumption that the valence holes have an infinite mass, thereby neglecting the influence of the recoil processes.

Chapter 4, contains a summary of the content of the two preceding chapters. In addition to that, the prospects for possible further theoretical studies are given on the basis of the results of this work.

Finally, two technical details concerning the presentation of the quantitative results should be mentioned. All units are chosen in such a way that Planck's constant is equal to $\hbar=1$. Apart from that, all quantities are expressed with the help of characteristic material constants. 


\section{Chapter 2}

\section{Phonon-Induced Relaxation in Optically Excited Semiconductors}

In this chapter the influence of the interaction between longitudinal optical phonons and electrons on the relaxation of optically excited electron-hole pairs in semiconductors is studied both analytically and numerically with the help of quantum kinetic equations. The investigations are focused on the comparison of the different methods of approximation which are used in order to take the electron-electron interaction into account.

The content of the chapter is organized as follows. Section 2.1 contains the presentation of the different components of the model Hamiltonian describing the semiconductor. In Section 2.2 the quantum kinetic equations for the interacting electronphonon system are formulated on the basis of the Heisenberg equations-of-motion where the interaction between the electrons and phonons is treated within the framework of the second order self-consistent Born approximation. As far as the contributions which are due to the electron-electron interaction are concerned, it is possible to distinguish between the mean field corrections to the one-particle Hamiltonian and the vertex corrections to the electron-phonon interaction which have already been mentioned in Chapter 1. Section 2.3 contains the derivation of correction terms which approximately take the impact of higher order electron-phonon scattering processes into account. In Section 2.4 an alternative system of quantum kinetic equations, which is derived with the help of non-equilibrium Green's functions [43], is presented where the electron-electron interaction is only treated within the framework of the HartreeFock approximation. The following two sections are dedicated to the investigation of the differences between the results which are obtained with the help of the kinetic equations from Section 2.2 and Section 2.4. In Section 2.5 the linear optical response

of the system to an external laser field is studied by calculating the linear susceptibility for excitonic and continuum excitations. In Section 2.6 the dynamics of the electronic and phononic densities is calculated for different detunings of the excitation pulse in the weak nonlinear regime. 


\subsection{The Hamiltonian}

In the following the electrons of the semiconductor are described by a one-dimensional tight-binding model with two bands whose Hamiltonian is given by

$$
H_{e}^{0}=\sum_{\sigma=c, v} \sum_{k} \epsilon_{\sigma ; k} \psi_{\sigma k}^{\dagger} \psi_{\sigma k}
$$

where $\psi_{\sigma k}$ and $\psi_{\sigma k}^{\dagger}$ represent the fermionic destruction and creation operators for the electrons of the valence band $(\sigma=v)$ and the conduction band $(\sigma=c)$. It is assumed that the system consists of $N$ elementary cells whose length is chosen to be equal to unity. If the one-electron eigenfunctions satisfy periodic boundary conditions, the quasi-momenta $k$ are determined by the relation

$$
k=\frac{2 \pi}{N} n \quad \text { with } \quad n \in \mathbb{Z} \quad \text { and } \quad-\frac{N}{2} \leq n<\frac{N}{2} .
$$

If the hopping is restricted to the nearest neighbors, the free electron dispersions for the two bands satisfy the equations

$$
\begin{aligned}
& \epsilon_{v ; k}=2 t_{v}(\cos (k)-1), \\
& \epsilon_{c ; k}=-2 t_{c}(\cos (k)-1)+\Delta
\end{aligned}
$$

where $t_{v}$ and $t_{c}$ are the positive matrix elements for the intersite transitions while $\Delta$ represents the energy gap between the bands.

The interaction of the electron system with the external electrical field is described by the operator

$$
H_{e}^{I}(t)=-E(t) \sum_{\sigma_{1} \sigma_{2}} \sum_{k_{1} k_{2}} d_{k_{1} k_{2}}^{\sigma_{1} \sigma_{2}} \psi_{\sigma_{1} k_{1}}^{\dagger} \psi_{\sigma_{2} k_{2}}
$$

within the framework of the dipole approximation [31]. If all intraband transitions are neglected and if the system is excited homogeneously, the dipole matrix elements satisfy the equations

$$
d_{k_{1} k_{2}}^{c v}=d_{k_{1} k_{2}}^{v c}=d \delta_{k_{1} ; k_{2}} \quad \text { and } \quad d_{k_{1} k_{2}}^{c c}=d_{k_{1} k_{2}}^{v v}=0 .
$$

During the first picoseconds after an external optical excitation the dynamics of the polarization and the carrier distribution is mainly affected by the scattering of electrons and longitudinal optical phonons whereas the interaction between electrons and acoustical phonons only influences the time behavior of the system during a later stage $(\sim 2-100 \mathrm{ps})$. Since the studies in this chapter are focused on the relaxation of optically excited semiconductors during the non-thermal regime [57], it is therefore justified to neglect the acoustical phonons entirely. The contribution of the free longitudinal optical phonons to the total Hamiltonian is taken into account by the operator

$$
H_{p h}^{0}=\sum_{q} \omega_{L O} b_{q}^{\dagger} b_{q}
$$


where $b_{q}$ and $b_{q}^{\dagger}$ denote the bosonic field operators of the phonon system. The part of the total Hamiltonian which describes the interaction between the electrons and the longitudinal optical phonons is given by

$$
H_{e p h}=\sum_{\sigma} \sum_{k} \sum_{q \neq 0} g_{q}^{\sigma} \psi_{\sigma k+q}^{\dagger} \psi_{\sigma k}\left\{b_{q}+b_{-q}^{\dagger}\right\}
$$

where the scattering processes which lead to electronic interband transitions have been neglected since the energy balance of these processes is highly unfavorable. In the following it is assumed that the electron-phonon interaction is described by the Holstein model $[2,3]$. The matrix elements $g_{q}^{\sigma}$ then satisfy the equation

$$
g_{q}^{\sigma}=\frac{g}{\sqrt{N}}
$$

where $g$ denotes the local interaction strength. The Hamiltonian which represents the electron-electron interaction satisfies the following equation

$$
H_{e e}=\frac{1}{2 !} \sum_{\sigma_{1} \sigma_{2}} \sum_{q} V_{\sigma_{1} \sigma_{2}}(|q|) \sum_{k_{1} k_{2}} \psi_{\sigma_{1} k_{1}+q}^{\dagger} \psi_{\sigma_{2} k_{2}-q}^{\dagger} \psi_{\sigma_{2} k_{2}} \psi_{\sigma_{1} k_{1}}
$$

where the scattering processes which lead to a change of the number of carriers in the different bands have also been neglected for reasons of energy conservation. In this chapter it is assumed that the electron-electron scattering matrix $V_{\sigma_{1} \sigma_{2}}(|q|)$ is given by

$$
V_{\sigma_{1} \sigma_{2}}(|q|)=\frac{U}{N}+2 \pi \frac{\tilde{U}}{N^{2}} \sum_{n=1}^{n<\frac{N}{2}} \frac{\cos (n q)}{\sin \left(\frac{n \pi}{N}\right)} .
$$

The first term is due to the on-site interaction while the second one represents the contribution of the long-range part of the electron-electron interaction. In order to avoid an instability of the semiconductor ground state with respect to charge density waves, the inequality

$$
U>\tilde{U} \frac{\frac{\pi}{N}}{\sin \left(\frac{\pi}{N}\right)} 2 \ln 2
$$

has to be satisfied [32]. The total Hamiltonian of the semiconductor can now be written as follows

$$
H(t)=H_{e}(t)+H_{p h}^{0}+H_{e p h}+H_{e e}
$$

where the different parts of the electronic Hamiltonian have been combined:

$$
H_{e}(t)=H_{e}^{0}+H_{e}^{I}(t)=\sum_{\sigma_{1} \sigma_{2}} \sum_{k} \varepsilon_{\sigma_{1} \sigma_{2} ; k}(t) \psi_{\sigma_{1} k}^{\dagger} \psi_{\sigma_{2} k}
$$


Although the movement of the electrons is restricted to one dimension, the excitation dynamics of this model should exhibit the same characteristic features as two- or three-dimensional semiconductor models. This has been shown explicitly for the relaxation dynamics of hot electrons where the time behavior of a simplified one-dimensional model $[40,48]$ is in good qualitative agreement with the dynamics of three-dimensional semiconductor models $[25,26,36]$. The restriction to a onedimensional model, however, is only justified as long as the attention is not focused on the quantitative aspects of the dynamics. In this context it should be mentioned that the results for the three-dimensional semiconductor were calculated neglecting the angular dependence of all quantities since it would be impossible to treat the problem numerically otherwise. Thus the original system was described by an effective onedimensional model. As a consequence thereof a number of effects (for example the influence of the non-equilibrium dynamics of the phonons) was not taken into account [36].

In the initial state, long before the system is excited by the external field, the electrons completely fill the valence band while the phonons are described by a thermal distribution with a lattice temperature $T$. The state of the system is then determined by the statistical operator

$$
\rho=|\Phi\rangle\langle\Phi| \otimes \frac{1}{Z^{p h}} e^{-\frac{1}{k_{B} T} H_{p h}^{0}} \quad \text { with } \quad|\Phi\rangle:=\prod_{k} \psi_{v k}^{\dagger}\left|0_{e}\right\rangle
$$

where $\left|0_{e}\right\rangle$ denotes the electronic vacuum while $Z^{p h}$ is the canonical partition function for the phonon system.

Unless stated otherwise the electron-hole mass ratio $\kappa=\frac{t_{v}}{t_{c}}$ is chosen to be equal to $\kappa=0.15$ in the numerical calculations, this ratio is close to the ratio in GaAs. The total width of both bands is always equal to $4\left(t_{c}+t_{v}\right)=5 \omega_{L O}$ allowing the observation of real phonon emission and absorption processes. Concerning the electronelectron interaction it is generally assumed that it only acts on-site where the local interaction strength is always given by $U=1.5 \omega_{L O}$. The restriction to the on-site interaction makes it possible to derive exact analytical expressions for the eigenstates and eigenvectors of the subspace with one conduction electron and one valence hole (see Appendix B). The numerical calculations in Section 2.6 will show that an additional long-range Coulomb interaction does not lead to a qualitative change of the dynamics. The local interaction strength of the electron-phonon interaction always satisfies the equation $g=0.25 \omega_{L O}$. The crystal temperature is chosen from the interval $0 \leq k_{B} T \leq 1.5 \omega_{L O}$, the corresponding Bose factors are then located within the range $0 \leq n_{B}\left(\omega_{L O}\right) \leq 1$ with $n_{B}(\omega)=\left(\exp \left(\frac{\omega}{k_{B} T}\right)-1\right)^{-1}$.

\subsection{Kinetic Equations}

In this section the dynamics of the semiconductor is described with the help of the Heisenberg equations-of-motions for the different sorts of density functions using an 
approximation scheme which was introduced by Fricke [42]. In a first step the density functions are recursively expressed in terms of correlation functions starting with the one-particle expectation values. The infinite hierarchy of equations-of-motions which is formed by these new functions is then truncated by retaining only the correlation functions up to a certain order. The remaining differential equations can either be solved numerically or studied analytically in order to determine the time behavior of the system after an optical excitation.

In the following the interest is focused on the dynamics of the interband polarization $\left\langle\psi_{v l}^{\dagger} \psi_{c l}\right\rangle_{t}$ and the distribution functions of the valence electrons and the conduction electrons $\left\langle\psi_{v l}^{\dagger} \psi_{v l}\right\rangle_{t}$ and $\left\langle\psi_{c l}^{\dagger} \psi_{c l}\right\rangle_{t}$ whose time behavior is reflected in the linear and the nonlinear optical properties of the system. Since the Hamiltonian $H(t)$ conserves the total number of electrons these one-particle densities coincide with their correlated parts. Their dynamics is determined by the equation

$$
\begin{aligned}
i \frac{d}{d t}\left\langle\psi_{\lambda_{1} l}^{\dagger} \psi_{\lambda_{2} l}\right\rangle_{t} & =\sum_{\sigma}\left\{\tilde{\varepsilon}_{\lambda_{2} \sigma ; l}(t)\left\langle\psi_{\lambda_{1} l}^{\dagger} \psi_{\sigma l}\right\rangle_{t}-\tilde{\varepsilon}_{\sigma \lambda_{1} ; l}(t)\left\langle\psi_{\sigma l}^{\dagger} \psi_{\lambda_{2} l}\right\rangle_{t}\right\} \\
& +\delta\left\langle\psi_{\lambda_{1} l}^{\dagger} \psi_{\lambda_{2} l}\right\rangle_{e p}+\delta\left\langle\psi_{\lambda_{1} l}^{\dagger} \psi_{\lambda_{2} l}\right\rangle_{e e}
\end{aligned}
$$

The coefficients of the one-particle energy matrix $\tilde{\varepsilon}_{\lambda_{1} \lambda_{2} ; l}(t)$ which appear in Equation 2.16 are composed of the corresponding coefficients of the bare energy matrix $\varepsilon_{\lambda_{1} \lambda_{2} ; l}(t)$ and the dynamical Hartree-Fock contributions of the electron-electron interaction:

$$
\begin{aligned}
\tilde{\varepsilon}_{\lambda_{1} \lambda_{2} ; l}(t) & =\varepsilon_{\lambda_{1} \lambda_{2} ; l}(t)+\delta_{\lambda_{1} ; \lambda_{2}} \sum_{\sigma} V_{\lambda_{1} \sigma}(0) \sum_{k}\left\langle\psi_{\sigma k}^{\dagger} \psi_{\sigma k}\right\rangle_{t} \\
& -\sum_{k} V_{\lambda_{1} \lambda_{2}}(|k-l|)\left\langle\psi_{\lambda_{2} k}^{\dagger} \psi_{\lambda_{1} k}\right\rangle_{t} .
\end{aligned}
$$

The two additional contributions on the right-hand side of Equation 2.16 represent corrections to the Hartree-Fock approximation. The first contribution describes the influence of the electron-phonon interaction on the dynamics of the function $\left\langle\psi_{\lambda_{1} l}^{\dagger} \psi_{\lambda_{2} l}\right\rangle_{t}$. It reads

$$
\begin{aligned}
\delta\left\langle\psi_{\lambda_{1} l}^{\dagger} \psi_{\lambda_{2} l}\right\rangle_{e p} & =\sum_{q \neq 0} g_{q}^{\lambda_{2}}\left\{\left\langle\psi_{\lambda_{1} l}^{\dagger} \psi_{\lambda_{2} l-q} b_{q}\right\rangle_{t}+\left\langle\psi_{\lambda_{1} l}^{\dagger} \psi_{\lambda_{2} l+q} b_{q}^{\dagger}\right\rangle_{t}\right\} \\
& -\sum_{q \neq 0} g_{q}^{\lambda_{1}}\left\{\left\langle\psi_{\lambda_{1} l-q}^{\dagger} \psi_{\lambda_{2} l} b_{q}^{\dagger}\right\rangle_{t}+\left\langle\psi_{\lambda_{1} l+q}^{\dagger} \psi_{\lambda_{2} l} b_{q}\right\rangle_{t}\right\}
\end{aligned}
$$

where the new functions which appear on the right-hand side of Equation 2.18 are referred to as first order phonon-assisted densities. Strictly speaking, these phononassisted densities should be expressed with the help of the corresponding correlation functions, for example

$$
\left\langle\psi_{\lambda_{1} l+p}^{\dagger} \psi_{\lambda_{2} l} b_{p}\right\rangle_{t}=\left\langle\psi_{\lambda_{1} l+p}^{\dagger} \psi_{\lambda_{2} l} b_{p}\right\rangle_{t}^{c}+\left\langle\psi_{\lambda_{1} l+p}^{\dagger} \psi_{\lambda_{2} l}\right\rangle_{t}\left\langle b_{p}\right\rangle_{t}
$$


if the kinetic equations are formulated within the framework of the formalism presented in Reference [42]. However, the expectation values $\left\langle b_{p}\right\rangle_{t}$ and $\left\langle b_{p}^{\dagger}\right\rangle_{t}$ vanish for $p \neq 0$ since the Hamiltonian $H(t)$ guarantees the conservation of the total quasi momentum modulo a reciprocal lattice vector. For this reason the complete first order phonon-assisted densities are identical with their correlated parts. The second contribution in Equation 2.16 is the collision term for the electron-electron scattering processes. It satisfies the equation

$$
\begin{aligned}
\delta\left\langle\psi_{\lambda_{1} l}^{\dagger} \psi_{\lambda_{2} l}\right\rangle_{e e} & =\sum_{\sigma} \sum_{k} \sum_{q} V_{\lambda_{2} \sigma}(|q|)\left\langle\psi_{\lambda_{1} l}^{\dagger} \psi_{\sigma k}^{\dagger} \psi_{\sigma k+q} \psi_{\lambda_{2} l-q}\right\rangle_{t}^{c} \\
& -\sum_{\sigma} \sum_{k} \sum_{q} V_{\sigma \lambda_{1}}(|q|)\left\langle\psi_{\lambda_{1} l+q}^{\dagger} \psi_{\sigma k-q}^{\dagger} \psi_{\sigma k} \psi_{\lambda_{2} l}\right\rangle_{t}^{c} .
\end{aligned}
$$

The correlation functions with four electronic field operators which appear on the righthand side of Equation 2.20 are defined by the following relation

$$
\begin{aligned}
& \left\langle\psi_{\lambda_{1} l_{1}+p}^{\dagger} \psi_{\lambda_{2} l_{2}-p}^{\dagger} \psi_{\lambda_{3} l_{2}} \psi_{\lambda_{4} l_{1}}\right\rangle_{t}^{c}=\left\langle\psi_{\lambda_{1} l_{1}+p}^{\dagger} \psi_{\lambda_{2} l_{2}-p}^{\dagger} \psi_{\lambda_{3} l_{2}} \psi_{\lambda_{4} l_{1}}\right\rangle_{t} \\
& -\delta_{p ; 0}\left\langle\psi_{\lambda_{1} l_{1}}^{\dagger} \psi_{\lambda_{4} l_{1}}\right\rangle_{t}\left\langle\psi_{\lambda_{2} l_{2}}^{\dagger} \psi_{\lambda_{3} l_{2}}\right\rangle_{t}+\delta_{p ; l_{2}-l_{1}}\left\langle\psi_{\lambda_{1} l_{2}}^{\dagger} \psi_{\lambda_{3} l_{2}}^{\dagger}\right\rangle_{t}\left\langle\psi_{\lambda_{2} l_{1}} \psi_{\lambda_{4} l_{1}}\right\rangle_{t}
\end{aligned}
$$

where the conservation of the total quasi momentum has already been taken into account. If both correction terms are neglected, the dynamics for one-particle densities with different quasi momenta is decoupled and the time behavior is described by the well-known semiconductor Bloch equations. If the electron-electron and the electronphonon collision terms are taken into account, it is possible to describe the dephasing and relaxation processes which cause the decay of the initially excited state of the system. While the formation of bound pairs of valence holes and conduction electrons (excitons) in the low density limit can already be described within the framework of the semiconductor Bloch equations the complete description of bound molecule-like complexes of two valence holes and two conduction electrons (biexcitons) requires the consideration of the higher order correlation functions from $\delta\left\langle\psi_{\lambda_{1} l}^{\dagger} \psi_{\lambda_{2} l}\right\rangle_{e e}$. These functions are also necessary for the description of the screening of the electron-electron interaction in the high density limit.

In the following the influence of the electron-phonon scattering processes on the relaxation of the excited semiconductor is placed at the center of interest. For this reason the electronic collision term in Equation 2.16 is neglected and the attention is now focused on the time behavior of the first order phonon-assisted densities, which is determined by the equation

$$
\begin{aligned}
i \frac{d}{d t}\left\langle\psi_{\lambda_{1} l+p}^{\dagger} \psi_{\lambda_{2} l} b_{p}\right\rangle_{t} & =\omega_{p}\left\langle\psi_{\lambda_{1} l+p}^{\dagger} \psi_{\lambda_{2} l} b_{p}\right\rangle_{t} \\
& +\sum_{\sigma}\left\{\tilde{\varepsilon}_{\lambda_{2} \sigma ; l}(t)\left\langle\psi_{\lambda_{1} l+p}^{\dagger} \psi_{\sigma l} b_{p}\right\rangle_{t}-\tilde{\varepsilon}_{\sigma \lambda_{1} ; l+p}(t)\left\langle\psi_{\sigma l+p}^{\dagger} \psi_{\lambda_{2} l} b_{p}\right\rangle_{t}\right\} \\
& +\delta\left\langle\psi_{\lambda_{1} l+p}^{\dagger} \psi_{\lambda_{2} l} b_{p}\right\rangle_{e p}+\delta\left\langle\psi_{\lambda_{1} l+p}^{\dagger} \psi_{\lambda_{2} l} b_{p}\right\rangle_{e e} .
\end{aligned}
$$


It is obtained with the help of the same factorization scheme which has already been applied above. As in Equation 2.16 the different contributions on the right-hand side of Equation 2.22 can be assigned to two different groups. The terms in the first two lines describe the renormalized one-particle dynamics within the framework of the HartreeFock approximation while the two expressions in the last line contain the higher order correction terms. The form of the correction term which is related to the electronphonon scattering processes is determined by the equation

$$
\begin{aligned}
\delta\left\langle\psi_{\lambda_{1} l+p}^{\dagger} \psi_{\lambda_{2} l} b_{p}\right\rangle_{e p} & =g_{p}^{\lambda_{2}}\left\langle\psi_{\lambda_{1} l+p}^{\dagger} \psi_{\lambda_{2} l+p}\right\rangle_{t}-\sum_{\sigma} g_{p}^{\sigma}\left\langle\psi_{\lambda_{1} l+p}^{\dagger} \psi_{\sigma l+p}\right\rangle_{t}\left\langle\psi_{\sigma l}^{\dagger} \psi_{\lambda_{2} l}\right\rangle_{t} \\
& +\left\{g_{p}^{\lambda_{2}}\left\langle\psi_{\lambda_{1} l+p}^{\dagger} \psi_{\lambda_{2} l+p}\right\rangle_{t}-g_{p}^{\lambda_{1}}\left\langle\psi_{\lambda_{1} l}^{\dagger} \psi_{\lambda_{2} l}\right\rangle_{t}\right\}\left\{\left\langle b_{p}^{\dagger} b_{p}\right\rangle_{t}+\left\langle b_{-p} b_{p}\right\rangle_{t}\right\} \\
& +\sum_{q \neq 0} g_{q}^{\lambda_{2}}\left\{\left\langle\psi_{\lambda_{1} l+p}^{\dagger} \psi_{\lambda_{2} l-q} b_{q} b_{p}\right\rangle_{t}^{c}+\left\langle\psi_{\lambda_{1} l+p}^{\dagger} \psi_{\lambda_{2} l+q} b_{q}^{\dagger} b_{p}\right\rangle_{t}^{c}\right\} \\
& -\sum_{q \neq 0} g_{q}^{\lambda_{1}}\left\{\left\langle\psi_{\lambda_{1} l+p+q}^{\dagger} \psi_{\lambda_{2} l} b_{q} b_{p}\right\rangle_{t}^{c}+\left\langle\psi_{\lambda_{1} l+p-q}^{\dagger} \psi_{\lambda_{2} l} b_{q}^{\dagger} b_{p}\right\rangle_{t}^{c}\right\} \\
& +\sum_{\sigma} \sum_{k} g_{p}^{\sigma}\left\langle\psi_{\lambda_{1} l+p}^{\dagger} \psi_{\sigma k}^{\dagger} \psi_{\sigma k+p} \psi_{\lambda_{2} l}\right\rangle_{t}^{c}
\end{aligned}
$$

while the contribution which is due to the electron-electron interaction satisfies the relation

$$
\begin{aligned}
\delta\left\langle\psi_{\lambda_{1} l+p}^{\dagger} \psi_{\lambda_{2} l} b_{p}\right\rangle_{e e} & =-\sum_{\sigma}\left\langle\psi_{\lambda_{1} l+p}^{\dagger} \psi_{\sigma l+p}\right\rangle_{t} \sum_{k} V_{\lambda_{2} \sigma}(|k-l|)\left\langle\psi_{\sigma k+p}^{\dagger} \psi_{\lambda_{2} k} b_{p}\right\rangle_{t} \\
& +\sum_{\sigma}\left\langle\psi_{\sigma l}^{\dagger} \psi_{\lambda_{2} l}\right\rangle_{t} \sum_{k} V_{\sigma \lambda_{1}}(|k-l|)\left\langle\psi_{\lambda_{1} k+p}^{\dagger} \psi_{\sigma k} b_{p}\right\rangle_{t} \\
& +\left\langle\psi_{\lambda_{1} l+p}^{\dagger} \psi_{\lambda_{2} l+p}\right\rangle_{t} \sum_{\sigma} \sum_{k} V_{\lambda_{2} \sigma}(|p|)\left\langle\psi_{\sigma k+p}^{\dagger} \psi_{\sigma k} b_{p}\right\rangle_{t} \\
& -\left\langle\psi_{\lambda_{1} l}^{\dagger} \psi_{\lambda_{2} l}\right\rangle_{t} \sum_{\sigma} \sum_{k} V_{\sigma \lambda_{1}}(|p|)\left\langle\psi_{\sigma k+p}^{\dagger} \psi_{\sigma k} b_{p}\right\rangle_{t} \\
& +\sum_{\sigma} \sum_{k} \sum_{q} V_{\lambda_{2} \sigma}(|q|)\left\langle\psi_{\lambda_{1} l+p}^{\dagger} \psi_{\sigma k}^{\dagger} \psi_{\sigma k+q} \psi_{\lambda_{2} l-q} b_{p}\right\rangle_{t}^{c} \\
& -\sum_{\sigma} \sum_{k} \sum_{q} V_{\sigma \lambda_{1}}(|q|)\left\langle\psi_{\lambda_{1} l+p+q}^{\dagger} \psi_{\sigma k-q}^{\dagger} \psi_{\sigma k} \psi_{\lambda_{2} l} b_{p}\right\rangle_{t}^{c} .
\end{aligned}
$$

The new functions which appear on the right-hand side of Equation 2.23 are the correlated parts of the so-called second order phonon-assisted densities. They are defined by the relations

$$
\left\langle\psi_{\lambda_{1} l-p_{1}+p_{2}}^{\dagger} \psi_{\lambda_{2} l} b_{p_{1}}^{\dagger} b_{p_{2}}\right\rangle_{t}^{c}=\left\langle\psi_{\lambda_{1} l-p_{1}+p_{2}}^{\dagger} \psi_{\lambda_{2} l} b_{p_{1}}^{\dagger} b_{p_{2}}\right\rangle_{t}-\delta_{p_{1} ; p_{2}}\left\langle\psi_{\lambda_{1} l}^{\dagger} \psi_{\lambda_{2} l}\right\rangle_{t}\left\langle b_{p_{1}}^{\dagger} b_{p_{1}}\right\rangle_{t}
$$

and

$$
\left\langle\psi_{\lambda_{1} l+p_{1}+p_{2}}^{\dagger} \psi_{\lambda_{2} l} b_{p_{1}} b_{p_{2}}\right\rangle_{t}^{c}=\left\langle\psi_{\lambda_{1} l+p_{1}+p_{2}}^{\dagger} \psi_{\lambda_{2} l} b_{p_{1}} b_{p_{2}}\right\rangle_{t}-\delta_{p_{1} ;-p_{2}}\left\langle\psi_{\lambda_{1} l}^{\dagger} \psi_{\lambda_{2} l}\right\rangle_{t}\left\langle b_{-p_{1}} b_{p_{1}}\right\rangle_{t} .
$$


The new sort of correlation function which has been introduced in Equation 2.24 is defined by the identity

$$
\begin{aligned}
& \left\langle\psi_{\lambda_{1} l_{1}+p_{1}+p_{2}}^{\dagger} \psi_{\lambda_{2} l_{2}-p_{2}}^{\dagger} \psi_{\lambda_{3} l_{2}} \psi_{\lambda_{4} l_{1}} b_{p_{1}}\right\rangle_{t}^{c}=\left\langle\psi_{\lambda_{1} l_{1}+p_{1}+p_{2}}^{\dagger} \psi_{\lambda_{2} l_{2}-p_{2}}^{\dagger} \psi_{\lambda_{3} l_{2}} \psi_{\lambda_{4} l_{1}} b_{p_{1}}\right\rangle_{t} \\
& -\delta_{p_{2} ; 0}\left\langle\psi_{\lambda_{2} l_{2}}^{\dagger} \psi_{\left.\lambda_{3} l_{2}\right\rangle_{t}}\left\langle\psi_{\lambda_{1} l_{1}+p_{1}}^{\dagger} \psi_{\lambda_{4} l_{1}} b_{p_{1}}\right\rangle_{t}+\delta_{p_{2} ; l_{2}-l_{1}-p_{1}}\left\langle\psi_{\lambda_{1} l_{2}}^{\dagger} \psi_{\lambda_{3} l_{2}}\right\rangle_{t}\left\langle\psi_{\lambda_{2} l_{1}+p_{1}}^{\dagger} \psi_{\lambda_{4} l_{1}} b_{p_{1}}\right\rangle_{t}\right. \\
& -\delta_{p_{2} ;-p_{1}}\left\langle\psi_{\lambda_{1} l_{1}}^{\dagger} \psi_{\lambda_{4} l_{1}}\right\rangle_{t}\left\langle\psi_{\lambda_{2} l_{2}+p_{1}}^{\dagger} \psi_{\lambda_{3} l_{2}} b_{p_{1}}\right\rangle_{t}+\delta_{p_{2} ; l_{2}-l_{1}}\left\langle\psi_{\lambda_{2} l_{1}}^{\dagger} \psi_{\lambda_{4} l_{1}}\right\rangle_{t}\left\langle\psi_{\lambda_{1} l_{2}+p_{1}}^{\dagger} \psi_{\lambda_{3} l_{2}} b_{p_{1}}\right\rangle_{t} .
\end{aligned}
$$

Apart from the electronic correlation functions the kinetic equations for the first order phonon-assisted density functions contain purely phononic densities, namely the phonon distribution function $\left\langle b_{p}^{\dagger} b_{p}\right\rangle_{t}$ and the two phonon coherence $\left\langle b_{-p} b_{p}\right\rangle_{t}$, which is also called phonon distortion [55]. The dynamics of these densities is determined by the two kinetic equations

$$
i \frac{d}{d t}\left\langle b_{p}^{\dagger} b_{p}\right\rangle_{t}=\sum_{\sigma} \sum_{k} g_{p}^{\sigma}\left\{\left\langle\psi_{\sigma k}^{\dagger} \psi_{\sigma k+p} b_{p}^{\dagger}\right\rangle_{t}-\left\langle\psi_{\sigma k+p}^{\dagger} \psi_{\sigma k} b_{p}\right\rangle_{t}\right\}
$$

and

$$
\begin{aligned}
i \frac{d}{d t}\left\langle b_{-p} b_{p}\right\rangle_{t} & =\left\{\omega_{p}+\omega_{-p}\right\}\left\langle b_{-p} b_{p}\right\rangle_{t} \\
& +\sum_{\sigma} \sum_{k} g_{p}^{\sigma}\left\{\left\langle\psi_{\sigma k-p}^{\dagger} \psi_{\sigma k} b_{-p}\right\rangle_{t}+\left\langle\psi_{\sigma k+p}^{\dagger} \psi_{\sigma k} b_{p}\right\rangle_{t}\right\} .
\end{aligned}
$$

The number of one-particle density functions $\left\langle\psi_{\lambda_{1} l}^{\dagger} \psi_{\lambda_{2} l}\right\rangle_{t},\left\langle b_{p}^{\dagger} b_{p}\right\rangle_{t}$ and $\left\langle b_{-p} b_{p}\right\rangle_{t}$ which have to be taken into account when solving the kinetic equations increases linearly with the system size $N$. On the other hand, the number of first order phononassisted density functions increases with $N^{2}$ while the number of the other electronic density functions which appear in Equations 2.16 to 2.29 increases with an exponent which is even larger. In order to keep the influence of the finite-size effects small, it is therefore necessary to truncate the system of kinetic equations at the present level. Consequently, the correlation functions in the last three lines of Equation 2.23 and the last two lines of Equation 2.24 are not taken into account explicitly in the following calculations. However, the influence of some of theses functions can be considered approximately in the form of correction terms. This will be explained in Section 2.3 in detail.

\subsection{Higher Order Corrections}

Several studies have shown that the one-particle densities can exhibit an unphysical time behavior, if the approximated quantum kinetic equations which have been derived in Section 2.2 are used for the description of the dynamics [36, 38]. For example, the electron distribution functions can become negative or larger than unity. In order to avoid this, it is necessary to consider correlation functions of higher order. As 
mentioned above, however, the system of differential equations would become too large for numerical calculations, if they were taken into account explicitly. For this reason they are only included implicitly in Equation 2.22 with the help of correction terms

$$
i \frac{d}{d t}\left\langle\psi_{\lambda_{1} l+p}^{\dagger} \psi_{\lambda_{2} l} b_{p}\right\rangle_{t}=\cdots+\left\{\delta \epsilon_{\lambda_{2} ; l}-\delta \epsilon_{\lambda_{1} ; l+p}^{*}\right\}\left\langle\psi_{\lambda_{1} l+p}^{\dagger} \psi_{\lambda_{2} l} b_{p}\right\rangle_{t}
$$

where the functions $\delta \epsilon_{c ; l}$ and $\delta \epsilon_{v ; l}$ will be defined below.

These correction terms are derived from the correlated parts of the second order phonon-assisted density functions which appear in the complete kinetic equation for the first order phonon-assisted densities:

$$
\begin{aligned}
i \frac{d}{d t}\left\langle\psi_{\lambda_{1} l+p}^{\dagger} \psi_{\lambda_{2} l} b_{p}\right\rangle_{t}=\cdots & +\sum_{q \neq 0} g_{q}^{\lambda_{2}}\left\{\left\langle\psi_{\lambda_{1} l+p}^{\dagger} \psi_{\lambda_{2} l-q} b_{q} b_{p}\right\rangle_{t}^{c}+\left\langle\psi_{\lambda_{1} l+p}^{\dagger} \psi_{\lambda_{2} l+q} b_{q}^{\dagger} b_{p}\right\rangle_{t}^{c}\right\} \\
& -\sum_{q \neq 0} g_{q}^{\lambda_{1}}\left\{\left\langle\psi_{\lambda_{1} l+p+q}^{\dagger} \psi_{\lambda_{2} l} b_{q} b_{p}\right\rangle_{t}^{c}+\left\langle\psi_{\lambda_{1} l+p-q}^{\dagger} \psi_{\lambda_{2} l} b_{q}^{\dagger} b_{p}\right\rangle_{t}^{c}\right\} .
\end{aligned}
$$

The kinetic equations for the functions which appear on the right-hand side of Equation 2.31 are listed in Appendix A. If they were considered explicitly in the numerical calculations, the computing time would increase with the third power of the size of the system and it would become difficult to compute the dynamics of realistic models. However, the qualitative influence of the correlated parts of the second order phononassisted densities can be estimated, if their time behavior is calculated with the help of simplified versions of the corresponding kinetic equations. In order to obtain these simplified equations, the exact kinetic equations are approximated in three steps. First the contributions which are due to the electron-electron interaction and the interaction between the electrons and the external field are neglected entirely. In a second step, all incoherent contributions are omitted. That means that only those terms which are proportional to $\left\langle\psi_{\lambda_{1} l+p}^{\dagger} \psi_{\lambda_{2} l} b_{p}\right\rangle_{t}$ are taken into account. The approximated kinetic equations for the functions which appear in the first line of Equation 2.31 then read

$$
\begin{gathered}
i \frac{d}{d t}\left\langle\psi_{\lambda_{1} l+p}^{\dagger} \psi_{\lambda_{2} l-q} b_{q} b_{p}\right\rangle_{t} \approx\left\{\omega_{p}+\omega_{q}+\epsilon_{\lambda_{2} ; l-q}-\epsilon_{\lambda_{1} ; l+p}\right\}\left\langle\psi_{\lambda_{1} l+p}^{\dagger} \psi_{\lambda_{2} l-q} b_{q} b_{p}\right\rangle_{t} \\
\quad+g_{q}^{\lambda_{2}}\left\{\left\langle b_{q}^{\dagger} b_{q}\right\rangle_{t}+\left\langle b_{-q} b_{q}\right\rangle_{t}+1-\left\langle\psi_{\lambda_{2} l-q}^{\dagger} \psi_{\lambda_{2} l-q}\right\rangle_{t}\right\}\left\langle\psi_{\lambda_{1} l+p}^{\dagger} \psi_{\lambda_{2} l} b_{p}\right\rangle_{t}
\end{gathered}
$$

and

$$
\begin{gathered}
i \frac{d}{d t}\left\langle\psi_{\lambda_{1} l+p}^{\dagger} \psi_{\lambda_{2} l+q} b_{q}^{\dagger} b_{p}\right\rangle_{t} \approx\left\{\omega_{p}-\omega_{q}+\epsilon_{\lambda_{2} ; l+q}-\epsilon_{\lambda_{1} ; l+p}\right\}\left\langle\psi_{\lambda_{1} l+p}^{\dagger} \psi_{\lambda_{2} l+q} b_{q}^{\dagger} b_{p}\right\rangle_{t} \\
+g_{q}^{\lambda_{2}}\left\{\left\langle b_{q}^{\dagger} b_{q}\right\rangle_{t}+\left\langle b_{q}^{\dagger} b_{-q}^{\dagger}\right\rangle_{t}+\left\langle\psi_{\lambda_{2} l+q}^{\dagger} \psi_{\lambda_{2} l+q}\right\rangle_{t}\right\}\left\langle\psi_{\lambda_{1} l+p}^{\dagger} \psi_{\lambda_{2} l} b_{p}\right\rangle_{t}
\end{gathered}
$$


whereas the correlation functions in the second line of Equation 2.31 satisfy the equations

$$
\begin{gathered}
i \frac{d}{d t}\left\langle\psi_{\lambda_{1} l+p+q}^{\dagger} \psi_{\lambda_{2} l} b_{q} b_{p}\right\rangle_{t} \approx\left\{\omega_{p}+\omega_{q}+\epsilon_{\lambda_{2} ; l}-\epsilon_{\lambda_{1} ; l+p+q}\right\}\left\langle\psi_{\lambda_{1} l+p+q}^{\dagger} \psi_{\lambda_{2} l} b_{q} b_{p}\right\rangle_{t} \\
-g_{q}^{\lambda_{1}}\left\{\left\langle b_{q}^{\dagger} b_{q}\right\rangle_{t}+\left\langle b_{-q} b_{q}\right\rangle_{t}+\left\langle\psi_{\lambda_{1} l+p+q}^{\dagger} \psi_{\lambda_{1} l+p+q}\right\rangle_{t}\right\}\left\langle\psi_{\lambda_{1} l+p}^{\dagger} \psi_{\lambda_{2} l} b_{p}\right\rangle_{t}
\end{gathered}
$$

and

$$
\begin{aligned}
i \frac{d}{d t}\left\langle\psi_{\lambda_{1} l+p-q}^{\dagger} \psi_{\lambda_{2} l} b_{q}^{\dagger} b_{p}\right\rangle_{t} \approx\left\{\omega_{p}-\omega_{q}+\epsilon_{\lambda_{2} ; l}-\epsilon_{\lambda_{1} ; l+p-q}\right\}\left\langle\psi_{\lambda_{1} l+p-q}^{\dagger} \psi_{\lambda_{2} l} b_{q}^{\dagger} b_{p}\right\rangle_{t} \\
-g_{q}^{\lambda_{1}}\left\{\left\langle b_{q}^{\dagger} b_{q}\right\rangle_{t}+\left\langle b_{q}^{\dagger} b_{-q}^{\dagger}\right\rangle_{t}+1-\left\langle\psi_{\lambda_{1} l+p-q}^{\dagger} \psi_{\lambda_{1} l+p-q}\right\rangle_{t}\right\}\left\langle\psi_{\lambda_{1} l+p}^{\dagger} \psi_{\lambda_{2} l} b_{p}\right\rangle_{t} .
\end{aligned}
$$

In the third step, the two-operator expectation values in Equations 2.32 to 2.35 are replaced by their initial values $\left\langle\psi_{\lambda l}^{\dagger} \psi_{\lambda l}\right\rangle_{t_{0}}=\delta_{\lambda ; v},\left\langle b_{p}^{\dagger} b_{p}\right\rangle_{t_{0}}=n_{B}\left(\omega_{L O}\right)$ and $\left\langle b_{-p} b_{p}\right\rangle_{t_{0}}=$ 0 . This approximation is justified, if the excitation of the semiconductor by the external field does not lead to considerable changes of the electron and phonon distributions. After the resulting differential equations have been integrated, the two expressions which appear on the right-hand side of Equation 2.31 satisfy the following relations

$$
\begin{aligned}
& \sum_{q \neq 0} g_{q}^{\lambda_{2}}\left\{\left\langle\psi_{\lambda_{1} l+p}^{\dagger} \psi_{\lambda_{2} l-q} b_{q} b_{p}\right\rangle_{t}^{c}+\left\langle\psi_{\lambda_{1} l+p}^{\dagger} \psi_{\lambda_{2} l+q} b_{q}^{\dagger} b_{p}\right\rangle_{t}^{c}\right\} \\
& =\int_{t_{0}}^{t} d \tau X_{\lambda_{2} ; l}(t-\tau) e^{-i\left\{\omega_{p}+\epsilon_{\lambda_{2} ; l}-\epsilon_{\lambda_{1} ; l+p}\right\}(t-\tau)}\left\langle\psi_{\lambda_{1} l+p}^{\dagger} \psi_{\lambda_{2} l} b_{p}\right\rangle_{\tau}
\end{aligned}
$$

and

$$
\begin{aligned}
& -\sum_{q \neq 0} g_{q}^{\lambda_{1}}\left\{\left\langle\psi_{\lambda_{1} l+p+q}^{\dagger} \psi_{\lambda_{2} l} b_{q} b_{p}\right\rangle_{t}^{c}+\left\langle\psi_{\lambda_{1} l+p-q}^{\dagger} \psi_{\lambda_{2} l} b_{q}^{\dagger} b_{p}\right\rangle_{t}^{c}\right\} \\
& =-\int_{t_{0}}^{t} d \tau X_{\lambda_{1} ; l+p}^{*}(t-\tau) e^{-i\left\{\omega_{p}+\epsilon_{\lambda_{2} ; l}-\epsilon_{\lambda_{1} ; l+p}\right\}(t-\tau)}\left\langle\psi_{\lambda_{1} l+p}^{\dagger} \psi_{\lambda_{2} l} b_{p}\right\rangle_{\tau}
\end{aligned}
$$

where the form of the memory functions is determined by the equation

$$
\begin{aligned}
X_{\lambda ; l}(t) & =(-i) \sum_{q \neq 0} g_{q}^{\lambda} g_{q}^{\lambda}\left\{n_{B}\left(\omega_{L O}\right)+\delta_{\lambda ; c}\right\} e^{-i\left\{\epsilon_{\lambda ; l+q}-\epsilon_{\lambda ; i}+\omega_{q}\right\} t} \\
& +(-i) \sum_{q \neq 0} g_{q}^{\lambda} g_{q}^{\lambda}\left\{n_{B}\left(\omega_{L O}\right)+\delta_{\lambda ; v}\right\} e^{-i\left\{\epsilon_{\lambda ; l+q}-\epsilon_{\lambda ; l}-\omega_{q}\right\} t} .
\end{aligned}
$$

If the thermodynamic limit is performed $(N \rightarrow \infty)$, the function $X_{\lambda ; l}$ reads

$$
\begin{aligned}
X_{\lambda ; l}(t) & =(-i)\left\{n_{B}\left(\omega_{L O}\right)+\delta_{c ; \lambda}\right\} \frac{g^{2}}{2 \pi} \int_{-\pi}^{\pi} d q e^{-i\left\{\epsilon_{\lambda ; q}-\epsilon_{\lambda ; l} l+\omega_{L O}\right\} t} \\
& +(-i)\left\{n_{B}\left(\omega_{L O}\right)+\delta_{v ; \lambda}\right\} \frac{g^{2}}{2 \pi} \int_{-\pi}^{\pi} d q e^{-i\left\{\epsilon_{\lambda ; q}-\epsilon_{\lambda ;} l-\omega_{L O}\right\} t} .
\end{aligned}
$$


The integrals can be calculated explicitly and one obtains the following compact expressions

$$
X_{c ; l}(t)=(-i) g^{2} e^{-i 2 t_{c} \cos (l) t} J_{0}\left(2 t_{c} t\right)\left\{\left\{n_{B}\left(\omega_{L O}\right)+1\right\} e^{-i \omega_{L O} t}+n_{B}\left(\omega_{L O}\right) e^{+i \omega_{L O} t}\right\}
$$

and

$$
X_{v ; l}(t)=(-i) g^{2} e^{+i 2 t_{v} \cos (l) t} J_{0}\left(2 t_{v} t\right)\left\{n_{B}\left(\omega_{L O}\right) e^{-i \omega_{L O} t}+\left\{n_{B}\left(\omega_{L O}\right)+1\right\} e^{+i \omega_{L O} t}\right\}
$$

where $J_{0}$ denotes the Bessel function of the first kind of order 0 . For large values of $t$ the memory function $X_{\lambda ; l}(t)$ oscillates harmonicly with an amplitude which decreases like the inverse square of $t$.

Each of the integrals in Equations 2.36 and 2.37 contains a fast oscillating exponential factor which compensates the free oscillations of the first order phonon-assisted densities. Consequently, the product of both terms is generally slowly variable in comparison with the function $X_{\lambda ; l}(t)$. Motivated by this fact, the Markov approximation is applied and the slowly varying parts of the integrals are evaluated at $\tau=t$. The resulting expressions can be simplified by replacing the time-dependent integrals with the time-independent constants

$$
\delta \epsilon_{\lambda ; l}=\lim _{t_{0} \rightarrow-\infty} \int_{t_{0}}^{t} d \tau X_{\lambda ; l}(t-\tau)
$$

since the first order phonon-assisted density functions only differ from zero for $t \gtrsim 0$ whereas $t_{0} \ll 0$. For $\lambda=c$ the time-independent correction term for the one-particle energies satisfies the equation

$$
\begin{aligned}
\delta \epsilon_{c ; l} & =\theta\left(\epsilon_{c ; l}+\omega_{L O}-\Delta-4 t_{c}\right) \frac{g^{2} n_{B}\left(\omega_{L O}\right)}{\sqrt{\left(\omega_{L O}-2 t_{c} \cos (l)\right)^{2}-4 t_{c}^{2}}} \\
& -\theta\left(\omega_{L O}+\Delta-\epsilon_{c ; l}\right) \frac{g^{2}\left\{1+n_{B}\left(\omega_{L O}\right)\right\}}{\sqrt{\left(\omega_{L O}+2 t_{c} \cos (l)\right)^{2}-4 t_{c}^{2}}} \\
& -i \theta\left(4 t_{c}+\Delta-\omega_{L O}-\epsilon_{c ; l}\right) \frac{g^{2} n_{B}\left(\omega_{L O}\right)}{\sqrt{4 t_{c}^{2}-\left(\omega_{L O}-2 t_{c} \cos (l)\right)^{2}}} \\
& -i \theta\left(\epsilon_{c ; l}-\omega_{L O}-\Delta\right) \frac{g^{2}\left\{1+n_{B}\left(\omega_{L O}\right)\right\}}{\sqrt{4 t_{c}^{2}-\left(\omega_{L O}+2 t_{c} \cos (l)\right)^{2}}} .
\end{aligned}
$$

The corresponding expression for $\delta \epsilon_{v ; l}$ can be calculated easily since $X_{v ; l}(t)$ has the same structure as $-X_{c ; l}^{*}(t)$.

In Figure 2.1 the function $\delta \epsilon_{c ; k}$ has been plotted for the temperatures $k_{B} T=0$ and $k_{B} T=0.4 \omega_{L O}$ and different quasi momenta $k$ in order to obtain an impression of its form. Since the only scattering processes at zero temperature are phonon emission 
processes the imaginary part of $\delta \epsilon_{c ; k}$, which is responsible for the damping of the dynamics of the first order phonon-assisted densities, vanishes, if the one-particle energy $\epsilon_{c ; k}$ is smaller than the threshold energy $\Delta+\omega_{L O}$ where the absolute value of the function $\delta \epsilon_{c ; k}$ diverges. If the temperature is nonzero, it is possible to observe an additional

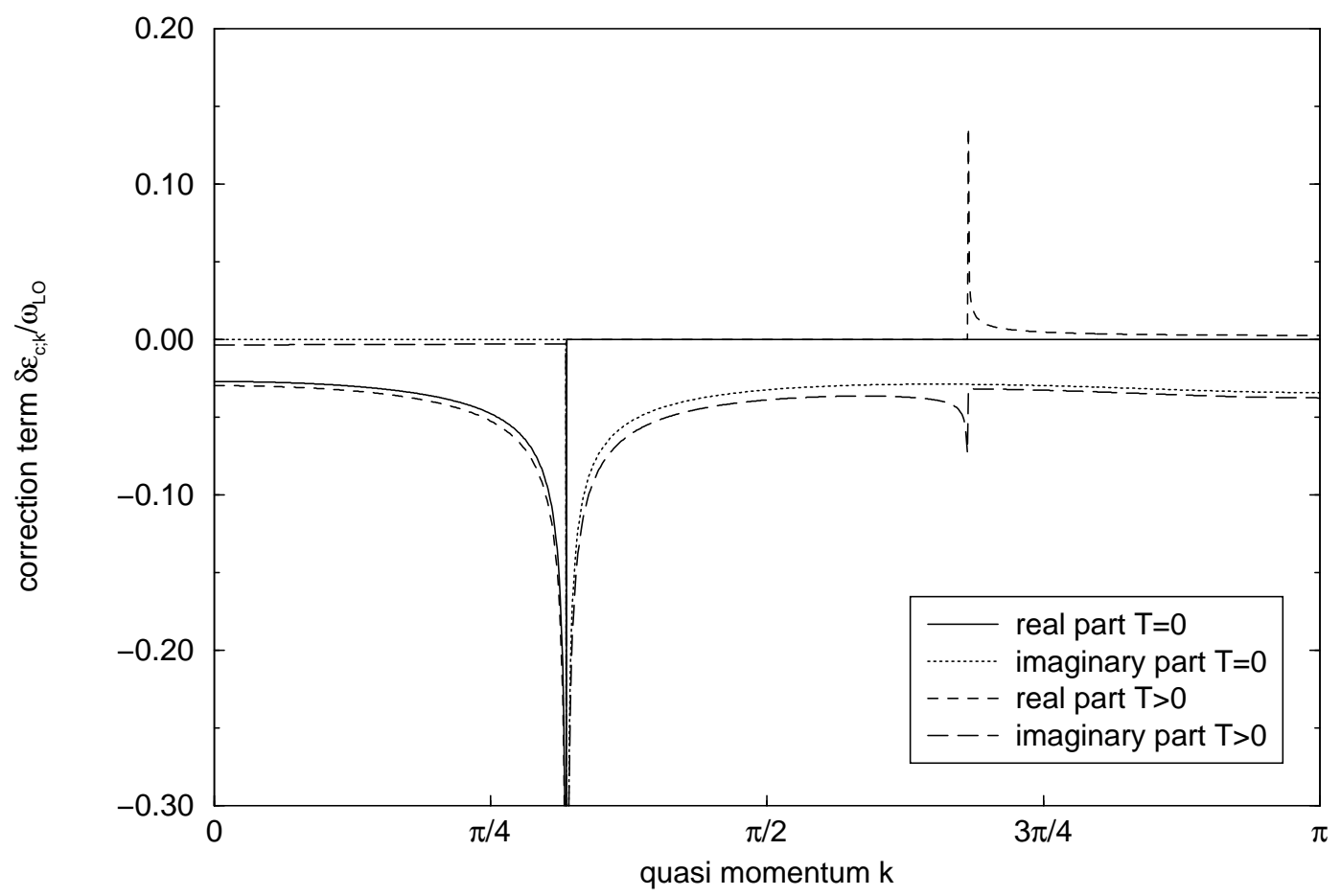

Figure 2.1: The correction term $\delta \epsilon_{c ; k}$ for $k_{B} T=0$ and $k_{B} T=0.4 \omega_{L O}$

threshold singularity at $4 t_{c}+\Delta-\omega_{L O}$ which is due to the phonon absorption processes. These processes are also responsible for the appearance of the small negative imaginary part below the phonon emission threshold. Apart from these features the form of $\delta \epsilon_{c ; k}$ is still similar to the corresponding function for $k_{B} T=0$ since the Bose factor $n_{B}\left(\omega_{L O}\right)$ for $k_{B} T=0.4 \omega_{L O}$ is considerably smaller than unity.

The divergence of the function $\delta \epsilon_{c ; k}$ at the threshold energies for the emission and absorption processes can be explained by the fact that the corresponding contributions to $X_{c ; k}(t)$ contain no oscillating factor which could guarantee the convergence of the integral in Equation 2.42. If the one-particle energy $\epsilon_{c ; k}$ is located in the vicinity of one of the threshold singularities, the weight of the memory function $X_{c ; k}(t)$ is shifted towards $t=-\infty$ and the usage of the Markov approximation poses a fundamental problem since the slowly varying factors which appear in the integrands in Equations 2.36 and 2.37 cannot simply be evaluated at $t=\tau$ anymore.

Numerical simulations, however, have shown that the behavior of the correction terms near the thresholds has only a minor impact on the dynamics of the system, as long as the real parts of $\delta \epsilon_{c ; k}$ and $\delta \epsilon_{v ; k}$ are neglected. For this reason, only the imaginary parts of the correction terms will be used in the following calculations in 
accordance with the usual procedure in the literature $[36,43,58]$. It will turn out that they suffice to avoid the unphysical results mentioned at the beginning of this section. A more consistent solution of the whole problem might be obtained, if the complete Non-Markovian expressions in Equations 2.36 and 2.37 were used.

\subsection{Green's Function Approach}

Non-equilibrium properties of many-body systems have been described with the help of real-time Green's function techniques since the beginning of the 1960s when Martin and Schwinger [4], Keldysh [8] and Kadanoff and Baym [5] developed the underlying formalism. During the last decade these techniques were employed extensively in order to study the dynamics of the interband polarization and the carrier distribution in semiconductors which are excited by strong ultrafast laser pulses [43]. In this section the description of the ultrafast time behavior of semiconductors on the basis of the non-equilibrium Green's function theory is outlined. The kinetic equations which are derived within the framework of this theory can then be compared with the approximated equations-of-motion from Section 2.2.

The unitary time-evolution operator, which determines the dynamics of the system, satisfies the differential equation

$$
i \frac{d}{d t} U\left(t, t_{0}\right)=H(t) U\left(t, t_{0}\right)
$$

with the initial condition

$$
U\left(t_{0}, t_{0}\right)=\mathbf{1}
$$

where the Hamiltonian

$$
H(t)=\tilde{H}(t)+V
$$

has already been presented in Section 2.1. The first operator on the right-hand side of Equation 2.46

$$
\tilde{H}(t)=H_{e}(t)+H_{p h}^{0}
$$

contains all one-particle contributions while the second operator

$$
V=H_{e p h}+H_{e e}
$$

describes the interaction between electrons and phonons. Within the framework of the time-dependent perturbation theory the time-evolution operator can be written as follows

$$
U\left(t, t_{0}\right)=\tilde{U}\left(t, t_{0}\right) T\left[e^{-i \int_{t_{0}}^{t} d \tau \tilde{V}(\tau)}\right]
$$


where $\tilde{U}\left(t, t_{0}\right)$ is the time-evolution operator for the non-interacting particles while $\tilde{V}$ denotes the operator $V$ in the interaction picture with respect to $\tilde{H}(t)$. The expression $T[\ldots]$ represents the time-ordering operator which arranges the operators so that the operator with the latest time stands farthest to the left. If $O$ is an arbitrary operator of the many-body-system, the following equation

$$
O(t)=U^{\dagger}\left(t, t_{0}\right) O U\left(t, t_{0}\right)=T_{\mathcal{C}}\left[\tilde{O}(t) e^{-i \int_{\mathcal{C}} d \tau \tilde{V}(\tau)}\right]
$$

holds. The integration is now performed along the Keldysh contour $\mathcal{C}$ which runs parallel to the real time axis from $t_{0}$ to $t$ and back (see Figure 2.2). The contourordering-operator $T_{\mathcal{C}}$ acts like $T$, but here the order is based on the position of the time variables on the Keldysh contour.

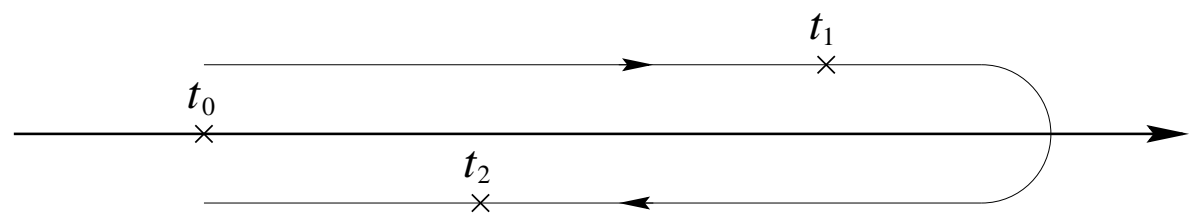

Figure 2.2: The Keldysh contour $\mathcal{C}$

The distribution functions for the electrons in the two bands and the inter-band polarization can be calculated with the help of the electron Green's function

$$
G_{\lambda_{1} \lambda_{2} ; l}\left(t_{1}, t_{2}\right)=(-i)\left\langle T_{\mathcal{C}}\left[\psi_{\lambda_{1} l}\left(t_{1}\right) \psi_{\lambda_{2} l}^{\dagger}\left(t_{2}\right)\right]\right\rangle
$$

while the phonon distribution function is related to the Green's function

$$
D_{p}\left(t_{1}, t_{2}\right)=(-i)\left\langle T_{\mathcal{C}}\left[b_{p}\left(t_{1}\right) b_{p}^{\dagger}\left(t_{2}\right)\right]\right\rangle .
$$

If the field operators which appear in Equations 2.51 and 2.52 are expressed with the help of the perturbative expansion from Equation 2.50, the following two relations

$$
G_{\lambda_{1} \lambda_{2} ; l}\left(t_{1}, t_{2}\right)=(-i)\left\langle T_{\mathcal{C}}\left[\tilde{\psi}_{\lambda_{1} l}\left(t_{1}\right) \tilde{\psi}_{\lambda_{2} l}^{\dagger}\left(t_{2}\right) e^{-i \int_{\mathcal{C}} d \tau \tilde{V}(\tau)}\right]\right\rangle
$$

and

$$
D_{p}\left(t_{1}, t_{2}\right)=(-i)\left\langle T_{\mathcal{C}}\left[\tilde{b}_{p}\left(t_{1}\right) \tilde{b}_{p}^{\dagger}\left(t_{2}\right) e^{-i \int_{\mathcal{C}} d \tau \tilde{V}(\tau)}\right]\right\rangle
$$

are obtained. In general, the expectation values of the products of field operators which appear on the right-hand sides of Equations 2.53 and 2.54 cannot be decomposed into products of the two-time Green's functions from Equations 2.51 and 2.52 because the statistical operator which describes the state of the many-body system at the initial time $t_{0}$ does not allow the application of Wick's theorem. In that case the initial values of the higher order correlation functions have to be taken into account when calculating the 
different terms of the perturbation series [37]. Fortunately, a Wick decomposition of all expectation values is possible within the framework of the model which is discussed here. This is due to the fact that the statistical operator for the phonon subsystem from Equation 2.15 is quadratic with respect to the operators $b_{p}$ and $b_{p}^{\dagger}$ while the initial state of the electron system is the free many-body ground state. Consequently, all techniques which have been developed for the calculation of equilibrium Green's functions can also be applied here.

The Green's function for the electrons then satisfies the following Dyson equations

$$
\begin{aligned}
G_{\lambda_{1} \lambda_{2} ; l}\left(t_{1}, t_{2}\right) & =\tilde{G}_{\lambda_{1} \lambda_{2} ; l}\left(t_{1}, t_{2}\right)+\sum_{\sigma_{1} \sigma_{2}} \int_{\mathcal{C}} d \tau \tilde{G}_{\lambda_{1} \sigma_{1} ; l}\left(t_{1}, \tau\right) \Sigma_{\sigma_{1} \sigma_{2} ; l}^{\delta}(\tau) G_{\sigma_{2} \lambda_{2} ; l}\left(\tau, t_{2}\right) \\
& +\sum_{\sigma_{1} \sigma_{2}} \int_{\mathcal{C}} d \tau_{1} \int_{\mathcal{C}} d \tau_{2} \tilde{G}_{\lambda_{1} \sigma_{1} ; l}\left(t_{1}, \tau_{1}\right) \Sigma_{\sigma_{1} \sigma_{2} ; l}\left(\tau_{1}, \tau_{2}\right) G_{\sigma_{2} \lambda_{2} ; l}\left(\tau_{2}, t_{2}\right)
\end{aligned}
$$

and

$$
\begin{aligned}
G_{\lambda_{1} \lambda_{2} ; l}\left(t_{1}, t_{2}\right) & =\tilde{G}_{\lambda_{1} \lambda_{2} ; l}\left(t_{1}, t_{2}\right)+\sum_{\sigma_{1} \sigma_{2}} \int_{\mathcal{C}} d \tau G_{\lambda_{1} \sigma_{1} ; l}\left(t_{1}, \tau\right) \Sigma_{\sigma_{1} \sigma_{2} ; l}^{\delta}(\tau) \tilde{G}_{\sigma_{2} \lambda_{2} ; l}\left(\tau, t_{2}\right) \\
& +\sum_{\sigma_{1} \sigma_{2}} \int_{\mathcal{C}} d \tau_{1} \int_{\mathcal{C}} d \tau_{2} G_{\lambda_{1} \sigma_{1} ; l}\left(t_{1}, \tau_{1}\right) \Sigma_{\sigma_{1} \sigma_{2} ; l}\left(\tau_{1}, \tau_{2}\right) \tilde{G}_{\sigma_{2} \lambda_{2} ; l}\left(\tau_{2}, t_{2}\right)
\end{aligned}
$$

where $\tilde{G}_{\lambda_{1} \lambda_{2} ; l}\left(t_{1}, t_{2}\right)$ denotes the free Green's function for non-interacting electrons. If the electron-hole interaction is taken into account within the framework of the HartreeFock approximation, the singular part of the self-energy satisfies the equation

$$
\sum_{\lambda_{1} \lambda_{2} ; l}^{\delta}(t)=\delta_{\lambda_{1} ; \lambda_{2}} \sum_{\sigma} V_{\lambda_{1} \sigma}(0) \sum_{k}\left\langle\psi_{\sigma k}^{\dagger} \psi_{\sigma k}\right\rangle_{t}-\sum_{k} V_{\lambda_{1} \lambda_{2}}(|l-k|)\left\langle\psi_{\lambda_{2} k}^{\dagger} \psi_{\lambda_{1} k}\right\rangle_{t} .
$$

The two terms on the right-hand side of Equation 2.57 are already known from Section 2.2 where they were introduced as Coulomb corrections to the one-particle energy matrix in Equation 2.17. If the electron-phonon interaction is treated in the self-consistent second order Born approximation, the non-singular part of the self-energy reads

$$
\Sigma_{\lambda_{1} \lambda_{2} ; l}\left(t_{1}, t_{2}\right)=\sum_{q \neq 0} g_{q}^{\lambda_{1}} g_{q}^{\lambda_{2}} i G_{\lambda_{1} \lambda_{2} ; l-q}\left(t_{1}, t_{2}\right)\left\{D_{q}\left(t_{1}, t_{2}\right)+D_{-q}\left(t_{2}, t_{1}\right)\right\} .
$$

In Figure 2.3 the diagrammatic representation of Equation 2.55 is depicted where the thick solid lines and the thick dashed lines denote the complete Green's functions for the electrons and phonons respectively whereas the thin lines denote the corresponding free Green's functions. The second and the third diagram on the right-hand side of the equation are the Hartree-Fock contributions of the electron-electron interaction, which is represented by the dotted lines. The fourth and the fifth diagram are 


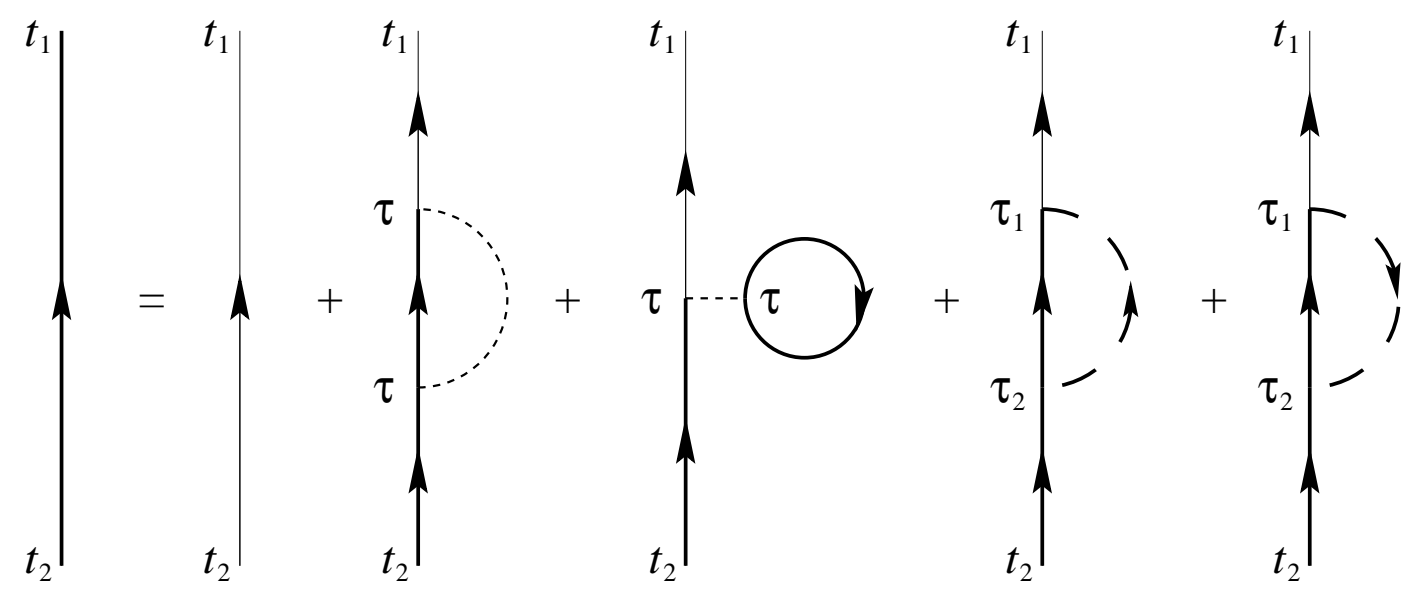

Figure 2.3: The Dyson equation for the Green's function of the electrons

due to the self-energy function from Equation 2.58. The corresponding diagrammatic representation for Equation 2.56 is obtained by replacing the external free Green's functions in the interaction diagrams with complete ones and vice versa.

The dynamics of the Green's function for the phonons can be determined by means of the following Dyson equations

$$
D_{p}\left(t_{1}, t_{2}\right)=\tilde{D}_{p}\left(t_{1}, t_{2}\right)+\int_{\mathcal{C}} d \tau_{1} \int_{\mathcal{C}} d \tau_{2} \tilde{D}_{p}\left(t_{1}, \tau_{1}\right) \Pi_{p}\left(\tau_{1}, \tau_{2}\right) D_{p}\left(\tau_{2}, t_{2}\right)
$$

and

$$
D_{p}\left(t_{1}, t_{2}\right)=\tilde{D}_{p}\left(t_{1}, t_{2}\right)+\int_{\mathcal{C}} d \tau_{1} \int_{\mathcal{C}} d \tau_{2} D_{p}\left(t_{1}, \tau_{1}\right) \Pi_{p}\left(\tau_{1}, \tau_{2}\right) \tilde{D}_{p}\left(\tau_{2}, t_{2}\right)
$$

where the polarization function $\Pi_{p}$ satisfies the relation

$$
\Pi_{p}\left(t_{1}, t_{2}\right)=-\sum_{\sigma_{1} \sigma_{2}} g_{p}^{\sigma_{1}} g_{p}^{\sigma_{2}} \sum_{k} i G_{\sigma_{1} \sigma_{2} ; k}\left(\tau_{1}, \tau_{2}\right) G_{\sigma_{2} \sigma_{1} ; k-q}\left(\tau_{2}, \tau_{1}\right)
$$

within the framework of the self-consistent Born approximation.The diagrams which correspond to the two terms in Equation 2.59 are depicted in Figure 2.4.

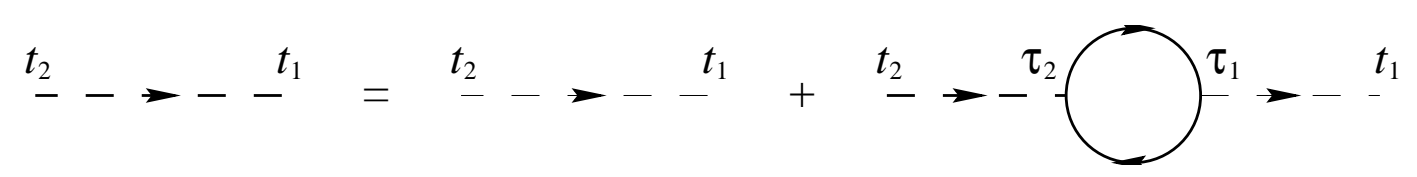

Figure 2.4: The Dyson equation for the Green's function of the phonons

The differential form of the Dyson equations can now be used for the derivation of a set of equations-of-motions for the density functions which is similar to the one 
which has been presented in Section 2.2. In the case of the Green's function of the electrons the differential equations read

$$
\begin{aligned}
i \frac{d}{d t_{1}} G_{\lambda_{1} \lambda_{2} ; l}\left(t_{1}, t_{2}\right) & =\delta_{\lambda_{1} ; \lambda_{2}} \delta_{\mathcal{C}}\left(t_{1}, t_{2}\right)+\sum_{\sigma} \tilde{\varepsilon}_{\lambda_{1} \sigma ; l}\left(t_{1}\right) G_{\sigma \lambda_{2} ; l}\left(t_{1}, t_{2}\right) \\
& +\sum_{\sigma} \int_{\mathcal{C}} d \tau \Sigma_{\lambda_{1} \sigma ; l}\left(t_{1}, \tau\right) G_{\sigma \lambda_{2} ; l}\left(\tau, t_{2}\right)
\end{aligned}
$$

and

$$
\begin{aligned}
i \frac{d}{d t_{2}} G_{\lambda_{1} \lambda_{2} ; l}\left(t_{1}, t_{2}\right) & =-\delta_{\lambda_{1} ; \lambda_{2}} \delta_{\mathcal{C}}\left(t_{1}, t_{2}\right)-\sum_{\sigma} \tilde{\varepsilon}_{\sigma \lambda_{2} ; l}\left(t_{2}\right) G_{\lambda_{1} \sigma ; l}\left(t_{1}, t_{2}\right) \\
& -\sum_{\sigma} \int_{\mathcal{C}} d \tau G_{\lambda_{1} \sigma ; l}\left(t_{1}, \tau\right) \Sigma_{\sigma \lambda_{2} ; l}\left(\tau, t_{2}\right) .
\end{aligned}
$$

If Equations 2.62 and 2.63 are combined, the following equation for the electronic densities

$$
\begin{aligned}
i \frac{d}{d t}\left\langle\psi_{\lambda_{2} l}^{\dagger} \psi_{\lambda_{1} l}\right\rangle_{t} & =\sum_{\sigma}\left\{\tilde{\varepsilon}_{\lambda_{1} \sigma ; l}(t)\left\langle\psi_{\lambda_{2} l}^{\dagger} \psi_{\sigma l}\right\rangle_{t}-\tilde{\varepsilon}_{\sigma \lambda_{2} ; l}(t)\left\langle\psi_{\sigma l}^{\dagger} \psi_{\lambda_{1} l}\right\rangle_{t}\right\} \\
& +(-i) \sum_{\sigma} \int_{t_{0}}^{t} d \tau\left\{\Sigma_{\lambda_{1} \sigma ; l}^{>}(t, \tau) G_{\sigma \lambda_{2} ; l}^{<}(\tau, t)-\Sigma_{\lambda_{1} \sigma ; l}^{<}(t, \tau) G_{\sigma \lambda_{2} ; l}^{>}(\tau, t)\right\} \\
& -(-i) \sum_{\sigma} \int_{t_{0}}^{t} d \tau\left\{G_{\lambda_{1} \sigma ; l}^{>}(t, \tau) \Sigma_{\sigma \lambda_{2} ; l}^{<}(\tau, t)-G_{\lambda_{1} \sigma ; l}^{<}(t, \tau) \Sigma_{\sigma \lambda_{2} ; l}^{>}(\tau, t)\right\}
\end{aligned}
$$

can be derived [43]. The corresponding equation for the density functions of the phonons is given by

$$
\begin{aligned}
i \frac{d}{d t}\left\langle b_{p}^{\dagger} b_{p}\right\rangle_{t} & =(-i) \int_{t_{0}}^{t} d \tau\left\{D_{p}^{>}(t, \tau) \Pi_{p}^{<}(\tau, t)-D_{p}^{<}(t, \tau) \Pi_{p}^{>}(\tau, t)\right\} \\
& -(-i) \int_{t_{0}}^{t} d \tau\left\{\Pi_{p}^{>}(t, \tau) D_{p}^{<}(\tau, t)-\Pi_{p}^{<}(t, \tau) D_{p}^{>}(\tau, t)\right\} .
\end{aligned}
$$

Unfortunately, this system of differential equations for the one-particle densities is not closed since the integrals on the right-hand sides of Equations 2.64 and 2.65 contain two-time Green's functions. In order to obtain a closed set of equations-of-motion for the density functions, it is necessary to eliminate the two-point Green's functions by means of an approximation method which is usually called Generalized KadanoffBaym Ansatz [18]. If this method is employed, the two-point Green's functions for the electrons are approximated with the help of the two relations

$$
G_{\lambda_{1} \lambda_{2} ; l}^{<}\left(t_{1}, t_{2}\right) \approx \sum_{\sigma}\left\{\left\langle\psi_{\sigma l}^{\dagger} \psi_{\lambda_{1} l}\right\rangle_{t_{1}} G_{\sigma \lambda_{2} ; l}^{a}\left(t_{1}, t_{2}\right)-G_{\lambda_{1} \sigma ; l}^{r}\left(t_{1}, t_{2}\right)\left\langle\psi_{\lambda_{2} l}^{\dagger} \psi_{\sigma l}\right\rangle_{t_{2}}\right\}
$$


and

$$
G_{\lambda_{1} \lambda_{2} ; l}^{>}\left(t_{1}, t_{2}\right) \approx \sum_{\sigma}\left\{G_{\lambda_{1} \sigma ; l}^{r}\left(t_{1}, t_{2}\right)\left\langle\psi_{\sigma l} \psi_{\lambda_{2} l}^{\dagger}\right\rangle_{t_{2}}-\left\langle\psi_{\lambda_{1} l} \psi_{\sigma l}^{\dagger}\right\rangle_{t_{1}} G_{\sigma \lambda_{2} ; l}^{a}\left(t_{1}, t_{2}\right)\right\}
$$

while the corresponding Green's functions for the phonons are replaced by the expressions

$$
D_{p}^{<}\left(t_{1}, t_{2}\right) \approx D_{p}^{r}\left(t_{1}, t_{2}\right)\left\langle b_{p}^{\dagger} b_{p}\right\rangle_{t_{2}}-\left\langle b_{p}^{\dagger} b_{p}\right\rangle_{t_{1}} D_{p}^{a}\left(t_{1}, t_{2}\right)
$$

and

$$
D_{p}^{>}\left(t_{1}, t_{2}\right) \approx D_{p}^{r}\left(t_{1}, t_{2}\right)\left\langle b_{p} b_{p}^{\dagger}\right\rangle_{t_{2}}-\left\langle b_{p} b_{p}^{\dagger}\right\rangle_{t_{1}} D_{p}^{a}\left(t_{1}, t_{2}\right) .
$$

The retarded and advanced Green's functions which appear in Equations 2.66 and 2.67 satisfy the equations

$$
G_{\lambda_{1} \lambda_{2} ; l}^{r}\left(t_{1}, t_{2}\right)=(-i) \theta\left(t_{1}-t_{2}\right)\left\{\left\langle\psi_{\lambda_{1} l}\left(t_{1}\right) \psi_{\lambda_{2} l}^{\dagger}\left(t_{2}\right)\right\rangle+\left\langle\psi_{\lambda_{2} l}^{\dagger}\left(t_{2}\right) \psi_{\lambda_{1} l}\left(t_{1}\right)\right\rangle\right\}
$$

and

$$
G_{\lambda_{1} \lambda_{2} ; l}^{a}\left(t_{1}, t_{2}\right)=(+i) \theta\left(t_{2}-t_{1}\right)\left\{\left\langle\psi_{\lambda_{1} l}\left(t_{1}\right) \psi_{\lambda_{2} l}^{\dagger}\left(t_{2}\right)\right\rangle+\left\langle\psi_{\lambda_{2} l}^{\dagger}\left(t_{2}\right) \psi_{\lambda_{1} l}\left(t_{1}\right)\right\rangle\right\} .
$$

The corresponding definitions for the phonon propagators $D^{r}$ and $D^{a}$ read

$$
D_{p}^{r}\left(t_{1}, t_{2}\right)=(-i) \theta\left(t_{1}-t_{2}\right)\left\{\left\langle b_{p}\left(t_{1}\right) b_{p}^{\dagger}\left(t_{2}\right)\right\rangle-\left\langle b_{p}^{\dagger}\left(t_{2}\right) b_{p}\left(t_{1}\right)\right\rangle\right\}
$$

and

$$
D_{p}^{a}\left(t_{1}, t_{2}\right)=(+i) \theta\left(t_{2}-t_{1}\right)\left\{\left\langle b_{p}\left(t_{1}\right) b_{p}^{\dagger}\left(t_{2}\right)\right\rangle-\left\langle b_{p}^{\dagger}\left(t_{2}\right) b_{p}\left(t_{1}\right)\right\rangle\right\} .
$$

The Generalized Kadanoff-Baym Ansatz gives the exact result, if the particles do not interact with each other. Otherwise the expressions in Equations 2.66 to 2.69 can be interpreted as the first terms in a perturbative expansion of the exact Green's functions where the correction terms take additional memory effects into account [18, 43].

The time behavior of the retarded and the advanced Green's function for the electrons is determined by the following differential equations

$$
\begin{aligned}
i \frac{d}{d t_{1}} G_{\lambda_{1} \lambda_{2} ; l}^{r}\left(t_{1}, t_{2}\right) & =\delta_{\lambda_{1} ; \lambda_{2}} \delta\left(t_{1}-t_{2}\right)+\sum_{\sigma} \tilde{\varepsilon}_{\lambda_{1} \sigma ; l}\left(t_{1}\right) G_{\sigma \lambda_{2} ; l}^{r}\left(t_{1}, t_{2}\right) \\
& +\sum_{\sigma} \int_{t_{2}}^{t_{1}} d \tau \Sigma_{\lambda_{1} \sigma ;}^{r}\left(t_{1}, \tau\right) G_{\sigma \lambda_{2} ; l}^{r}\left(\tau, t_{2}\right)
\end{aligned}
$$

and

$$
\begin{aligned}
i \frac{d}{d t_{2}} G_{\lambda_{1} \lambda_{2} ; l}^{a}\left(t_{1}, t_{2}\right) & =-\delta_{\lambda_{1} ; \lambda_{2}} \delta\left(t_{1}-t_{2}\right)-\sum_{\sigma} \tilde{\varepsilon}_{\sigma \lambda_{2} ; l}\left(t_{2}\right) G_{\lambda_{1} \sigma ; l}^{a}\left(t_{1}, t_{2}\right) \\
& -\sum_{\sigma} \int_{t_{1}}^{t_{2}} d \tau \Sigma_{\sigma \lambda_{2} ; l}^{a}\left(\tau, t_{2}\right) G_{\lambda_{1} \sigma ; l}^{a}\left(t_{1}, \tau\right) .
\end{aligned}
$$


The derivation of these equations proceeds in the same way as the derivation of the corresponding expressions for the complete Green's function in Equations 2.62 and 2.63. Instead of solving the full equations self-consistently, however, the retarded and the advanced self-energy, which appear as memory kernels in the integrals on the right-hand sides of Equations 2.74 and 2.75, are approximated by the corresponding free expressions

$$
\sum_{\lambda_{1} \lambda_{2} ; l}^{r ; 0}\left(t_{1}, t_{2}\right)=\theta\left(t_{1}-t_{2}\right) \delta_{\lambda_{1} ; \lambda_{2}} e^{-i \epsilon_{\lambda_{1} ; l}\left\{t_{1}-t_{2}\right\}} X_{\lambda_{1} ; l}\left(t_{1}-t_{2}\right)
$$

and

$$
\Sigma_{\lambda_{1} \lambda_{2} ; l}^{a ; 0}\left(t_{1}, t_{2}\right)=\theta\left(t_{2}-t_{1}\right) \delta_{\lambda_{1} ; \lambda_{2}} e^{i \epsilon_{\lambda_{1} ; l}\left\{t_{2}-t_{1}\right\}} X_{\lambda_{1} ; l}^{*}\left(t_{2}-t_{1}\right) .
$$

Consequently, the integrals from Equations 2.74 and 2.75, which describe the influence of the phonons on the electron relaxation, satisfy the following equations

$$
\sum_{\sigma} \int_{t_{2}}^{t_{1}} d \tau \sum_{\lambda_{1} \sigma ; l}^{r}\left(t_{1}, \tau\right) G_{\sigma \lambda_{2} ; l}^{r}\left(\tau, t_{2}\right) \approx \int_{t_{2}}^{t_{1}} d \tau X_{\lambda_{1} ; l}\left(t_{1}-\tau\right) e^{-i \epsilon_{\lambda_{1} ; l}\left\{t_{1}-\tau\right\}} G_{\lambda_{1} \lambda_{2} ; l}^{r}\left(\tau, t_{2}\right)
$$

and

$$
\sum_{\sigma} \int_{t_{1}}^{t_{2}} d \tau \Sigma_{\sigma \lambda_{2} ; l}^{a}\left(\tau, t_{2}\right) G_{\lambda_{1} \sigma ;}^{a}\left(t_{1}, \tau\right) \approx \int_{t_{1}}^{t_{2}} d \tau X_{\lambda_{2} ; l}^{*}\left(t_{2}-\tau\right) e^{i \epsilon_{\lambda_{2} ; l}\left\{t_{2}-\tau\right\}} G_{\lambda_{1} \lambda_{2} ; l}^{a}\left(t_{1}, \tau\right) .
$$

They have a similar structure as the integrals in Equations 2.36 and 2.37 where the fast oscillating exponential factors in the integrands compensate the free oscillating parts of the retarded and the advanced Green's function. It is now possible to perform the same approximations as in Section 2.3 which lead to the renormalization of the one-electron eigenenergies and the appearance of damping terms. As far as the retarded and the advanced phonon Green's function are concerned, it is assumed that the influence of the electrons can be neglected entirely and that the full propagators can be replaced by the free ones.

After having performed these approximations the kinetic equations for the electronic density functions have a similar form as the expression in Equation 2.16, if the electron-electron scattering corrections are neglected. However, the first order phononassisted densities are now defined by the relation

$$
\begin{aligned}
\left\langle\psi_{\lambda_{1} l+p}^{\dagger} \psi_{\lambda_{2} l} b_{p}\right\rangle_{t}= & \sum_{\varrho_{1} \varrho_{2}} \int_{t_{0}}^{t} d \tau D_{p}^{r}(t, \tau) G_{\lambda_{2} \varrho_{2} ; l}^{r}(t, \tau) G_{\varrho_{1} \lambda_{1} ; l+p}^{a}(\tau, t) \\
& \times\left\{g_{p}^{\varrho_{2}}\left\langle\psi_{\varrho_{1} l+p}^{\dagger} \psi_{\varrho_{2} l+p}\right\rangle_{\tau}-g_{p}^{\varrho_{1}}\left\langle\psi_{\varrho_{1} l}^{\dagger} \psi_{\varrho_{2} l}\right\rangle_{\tau}\right\}\left\langle b_{p}^{\dagger} b_{p}\right\rangle_{\tau} \\
+ & \sum_{\varrho_{1} \varrho_{2}} \int_{t_{0}}^{t} d \tau D_{p}^{r}(t, \tau) G_{\lambda_{2} \varrho_{2} ; l}^{r}(t, \tau) G_{\varrho_{1} \lambda_{1} ; l+p}^{a}(\tau, t) \\
& \times\left\{g_{p}^{\varrho_{2}}\left\langle\psi_{\varrho_{1} l+p}^{\dagger} \psi_{\varrho_{2} l+p}\right\rangle_{\tau}-\sum_{\sigma} g_{p}^{\sigma}\left\langle\psi_{\varrho_{1} l+p}^{\dagger} \psi_{\sigma l+p}\right\rangle_{\tau}\left\langle\psi_{\sigma l}^{\dagger} \psi_{\varrho_{2} l}\right\rangle_{\tau}\right\}
\end{aligned}
$$


By differentiating both sides with respect to $t$ the following kinetic equation

$$
\begin{aligned}
i \frac{d}{d t}\left\langle\psi_{\lambda_{1} l+p}^{\dagger} \psi_{\lambda_{2} l} b_{p}\right\rangle_{t} & =\omega_{p}\left\langle\psi_{\lambda_{1} l+p}^{\dagger} \psi_{\lambda_{2} l} b_{p}\right\rangle_{t} \\
& +\sum_{\sigma}\left\{\tilde{\varepsilon}_{\lambda_{2} \sigma ; l}(t)\left\langle\psi_{\lambda_{1} l+p}^{\dagger} \psi_{\sigma l} b_{p}\right\rangle_{t}-\tilde{\varepsilon}_{\sigma \lambda_{1} ; l+p}(t)\left\langle\psi_{\sigma l+p}^{\dagger} \psi_{\lambda_{2} l} b_{p}\right\rangle_{t}\right\} \\
& +\left\{g_{p}^{\lambda_{2}}\left\langle\psi_{\lambda_{1} l+p}^{\dagger} \psi_{\lambda_{2} l+p}\right\rangle_{t}-g_{p}^{\lambda_{1}}\left\langle\psi_{\lambda_{1} l}^{\dagger} \psi_{\lambda_{2} l}\right\rangle_{t}\right\}\left\langle b_{p}^{\dagger} b_{p}\right\rangle_{t} \\
& +g_{p}^{\lambda_{2}}\left\langle\psi_{\lambda_{1} l+p}^{\dagger} \psi_{\lambda_{2} l+p}\right\rangle_{t}-\sum_{\sigma} g_{p}^{\sigma}\left\langle\psi_{\lambda_{1} l+p}^{\dagger} \psi_{\sigma l+p}\right\rangle_{t}\left\langle\psi_{\sigma l}^{\dagger} \psi_{\lambda_{2} l}\right\rangle_{t} \\
& +\left\{\delta \epsilon_{\lambda_{2} ; l}-\delta \epsilon_{\lambda_{1} ; l+p}^{*}\right\}\left\langle\psi_{\lambda_{1} l+p}^{\dagger} \psi_{\lambda_{2} l} b_{p}\right\rangle_{t}
\end{aligned}
$$

can be obtained. The time behavior of the phonon distribution function which appears on the right-hand side of Equation 2.81 is determined by the same differential equation as in Section 2.2.

The system of kinetic equations which has been derived in this section corresponds to a system of kinetic equations which has been derived by means of the equationsof-motion method, if the electron-electron interaction is treated within the framework of the Hartree-Fock approximation [36]. In comparison with the results from Section 2.2 two differences can be observed. First, the products of first order phononassisted densities and ordinary electronic densities which appear in the collision term $\delta\left\langle\psi_{\lambda_{1} l+p}^{\dagger} \psi_{\lambda_{2} l} b_{p}\right\rangle_{e e}$ in Equation 2.24 do not appear on the right-hand side of Equation 2.81. In the language of Feynman diagrams these terms can be identified as corrections to the electron-phonon interaction vertex which are due to multiple electron-electron scattering processes [54]. In addition to that the two phonon coherence $\left\langle b_{-p} b_{p}\right\rangle_{t}$ is missing. However, the absolute value of this function is usually small in comparison with the phonon distribution function. Therefore the omission of the two phonon coherence should not alter the dynamics of the system significantly. In this context it should also be mentioned that the time behavior of the phonon functions is often neglected completely by replacing the time dependent functions with their initial values [36, 43] without changing the results of the numerical calculations substantially. The consequences of the neglect of the vertex corrections, however, have not been the object of a closer investigation, yet. In the following, the dynamics of the system will therefore be calculated by using the kinetic equations from Section 2.2 with and without vertex corrections in order to find out if significant differences exist between them.

\subsection{Linear Response}

It turns out to be useful to linearize the kinetic equations from Section 2.2 with respect to $E(t)$ in order to achieve a better understanding of the influence of the vertex corrections. Within this approximation only the off-diagonal elements of the density matrix 
are dynamical quantities. The time behavior of the polarization $\left\langle\psi_{v l}^{\dagger} \psi_{c l}\right\rangle_{t}$ is determined by the relation

$$
\begin{aligned}
i \frac{d}{d t}\left\langle\psi_{v l}^{\dagger} \psi_{c l}\right\rangle_{t} & =\left\{\epsilon_{c ; l}-\epsilon_{v ; l}+\sum_{k}\left\{V_{v v}(|k|)+V_{c v}(0)-V_{v v}(0)\right\}\right\}\left\langle\psi_{v l}^{\dagger} \psi_{c l}\right\rangle_{t} \\
& -\sum_{k} V_{c v}(|l-k|)\left\langle\psi_{v k}^{\dagger} \psi_{c k}\right\rangle_{t}-d E(t) \\
& +\sum_{q \neq 0}\left\{g_{q}^{c}\left\langle\psi_{v l}^{\dagger} \psi_{c l-q} b_{q}\right\rangle_{t}-g_{q}^{v}\left\langle\psi_{v l+q}^{\dagger} \psi_{c l} b_{q}\right\rangle_{t}\right\} \\
& +\sum_{q \neq 0}\left\{g_{q}^{c}\left\langle\psi_{v l}^{\dagger} \psi_{c l+q} b_{q}^{\dagger}\right\rangle_{t}-g_{q}^{v}\left\langle\psi_{v l-q}^{\dagger} \psi_{c l} b_{q}^{\dagger}\right\rangle_{t}\right\} .
\end{aligned}
$$

The first order phonon-assisted densities which appear as source terms on the righthand side of Equation 2.82 satisfy the equations

$$
\begin{aligned}
i \frac{d}{d t}\left\langle\psi_{v l-p}^{\dagger} \psi_{c l} b_{p}^{\dagger}\right\rangle_{t} & =-\omega_{L O}\left\langle\psi_{v l-p}^{\dagger} \psi_{c l} b_{p}^{\dagger}\right\rangle_{t} \\
& +\left\{\epsilon_{c ; l}-\epsilon_{v ; l-p}+\sum_{k}\left\{V_{v v}(|k|)+V_{c v}(0)-V_{v v}(0)\right\}\right\}\left\langle\psi_{v l-p}^{\dagger} \psi_{c l} b_{p}^{\dagger}\right\rangle_{t} \\
& +n_{B}\left(\omega_{L O}\right)\left\{g_{p}^{c}\left\langle\psi_{v l-p}^{\dagger} \psi_{c l-p}\right\rangle_{t}-g_{p}^{v}\left\langle\psi_{v l}^{\dagger} \psi_{c l}\right\rangle_{t}\right\} \\
& -\sum_{k} V_{c v}(|l-k|)\left\langle\psi_{v k-p}^{\dagger} \psi_{c k} b_{p}^{\dagger}\right\rangle_{t}
\end{aligned}
$$

and

$$
\begin{aligned}
i \frac{d}{d t}\left\langle\psi_{v l+p}^{\dagger} \psi_{c l} b_{p}\right\rangle_{t} & =\omega_{L O}\left\langle\psi_{v l+p}^{\dagger} \psi_{c l} b_{p}\right\rangle_{t} \\
& +\left\{\epsilon_{c ; l}-\epsilon_{v ; l+p}+\sum_{k}\left\{V_{v v}(|k|)+V_{c v}(0)-V_{v v}(0)\right\}\right\}\left\langle\psi_{v l+p}^{\dagger} \psi_{c l} b_{p}\right\rangle_{t} \\
& +\left\{1+n_{B}\left(\omega_{L O}\right)\right\}\left\{g_{p}^{c}\left\langle\psi_{v l+p}^{\dagger} \psi_{c l+p}\right\rangle_{t}-g_{p}^{v}\left\langle\psi_{v l}^{\dagger} \psi_{c l}\right\rangle_{t}\right\} \\
& -\sum_{k} V_{c v}(|l-k|)\left\langle\psi_{v k+p}^{\dagger} \psi_{c k} b_{p}\right\rangle_{t}
\end{aligned}
$$

where the correction terms have been omitted since they would not affect the results of the following investigations. The optical states with no center-of-mass momentum $\left\langle\psi_{v l}^{\dagger} \psi_{c l}\right\rangle_{t}$ are coupled to the two subspaces which are formed by the so-called dark states $\left\langle\psi_{v l+p}^{\dagger} \psi_{c l} b_{p}\right\rangle_{t}$ and $\left\langle\psi_{v l-p}^{\dagger} \psi_{c l} b_{p}^{\dagger}\right\rangle_{t}$ by phonon emission and absorption processes respectively. While the Hartree-Fock contributions lead to a constant shift of the free one-particle energies the vertex corrections are responsible for the last terms on the right-hand sides of Equation 2.83 and Equation 2.84 which are necessary for the correct description of the excitonic scattering dynamics in the two dark subspaces. Without them the pairs of conduction electrons and valence holes with a non-vanishing 
center-of-mass momentum would be treated as non-interacting particles unlike the optically excited electron-hole pairs.

The kinetic equations for the first order phonon-assisted densities are equivalent to the following integral equations

$$
\begin{aligned}
\left\langle\psi_{v l+p}^{\dagger} \psi_{c l} b_{p}\right\rangle_{t} & =(-i) \frac{g}{\sqrt{N}}\left\{1+n_{B}\left(\omega_{L O}\right)\right\} \\
& \times \sum_{k} \int_{t_{0}}^{t} d \tau\left\{G_{l ; k-p}^{+}(p ; t-\tau)-G_{l ; k}^{+}(p ; t-\tau)\right\}\left\langle\psi_{v k}^{\dagger} \psi_{c k}\right\rangle_{\tau}
\end{aligned}
$$

and

$$
\begin{aligned}
\left\langle\psi_{v l-p}^{\dagger} \psi_{c l} b_{p}^{\dagger}\right\rangle_{t} & =(-i) \frac{g}{\sqrt{N}} n_{B}\left(\omega_{L O}\right) \\
& \times \sum_{k} \int_{t_{0}}^{t} d \tau\left\{G_{l ; k+p}^{-}(p ; t-\tau)-G_{l ; k}^{-}(p ; t-\tau)\right\}\left\langle\psi_{v k}^{\dagger} \psi_{c k}\right\rangle_{\tau}
\end{aligned}
$$

The Green's functions in Equations 2.85 and 2.86 describe the dynamics of the first order phonon-assisted densities in the absence of the electron-phonon interaction. They obey the following differential equation

$$
i \frac{d}{d t} G_{l_{1} ; l_{2}}^{ \pm}(p ; t)=\sum_{k} \Omega_{l_{1} ; k}^{ \pm}(p) G_{k ; l_{2}}^{ \pm}(p ; t)
$$

with the initial condition

$$
G_{l_{1} ; l_{2}}^{ \pm}(p ; 0)=\delta_{l_{1} ; l_{2}} .
$$

If the long-range part of the electron-electron interaction in Equation 2.11 vanishes, the matrix elements read

$$
\Omega_{l_{1} ; l_{2}}^{ \pm}(p)=\delta_{l_{1} ; l_{2}}\left\{E\left(l_{1}, \pm p\right) \pm \omega_{L O}\right\}-\frac{U}{N}
$$

The function $E(l, p)$ is defined by the relation

$$
\begin{aligned}
E(l, p) & =\epsilon_{c ; l}-\epsilon_{v ; l+p}+U \\
& =E_{G}(U)-T(p) \cos (l+\varphi(p))
\end{aligned}
$$

and describes the energy of an excited electron-hole pair whose total momentum is equal to $-p$ where the band gap is shifted because of the electron-electron interaction. The generalized hopping element $T(p)$ and the phase shift function $\varphi(p)$ satisfy the equations

$$
T(p)=2 \sqrt{t_{v}^{2}+t_{c}^{2}+2 t_{c} t_{v} \cos (p)}
$$


and

$$
\varphi(p)=\arctan \left(\frac{t_{v} \sin (p)}{t_{c}+t_{v} \cos (p)}\right)
$$

while the function $E_{G}(U)$, which determines the position of $E(l, p)$ in the energy spectrum, is given by

$$
E_{G}(U)=2 t_{c}+2 t_{v}+\Delta+U
$$

In order to solve the differential equation for $G_{l_{1} ; l_{2}}^{ \pm}(p ; t)$, it is sufficient to solve the corresponding eigenvalue problem for a conduction electron and a valence hole in the presence of an attractive interaction. If the interaction function has only an on-site component, the eigenenergies and eigenstates can be calculated analytically and the explicit form of the Green's functions is known:

$$
\begin{aligned}
& G_{l_{1} ; l_{2}}^{+}(p ; t)=e^{-i \omega_{L O} t} G_{l_{1} ; l_{2}}(p ; t)=e^{-i \omega_{L O} t}\left\langle l_{1}, p\left|e^{-i t H(p)}\right| l_{2}, p\right\rangle, \\
& G_{l_{1} ; l_{2}}^{-}(p ; t)=e^{+i \omega_{L O} t} G_{l_{1} ; l_{2}}(-p ; t)=e^{+i \omega_{L O} t}\left\langle l_{1},-p\left|e^{-i t H(p)}\right| l_{2},-p\right\rangle .
\end{aligned}
$$

The definitions for the expressions which appear on the right-hand sides of Equations 2.94 and 2.95 can be found in Appendix B together with the complete analytical solution of the eigenvalue problem. If the long-range interaction parameter $\tilde{U}$ did not vanish, the differential equation for the Green's functions could only be solved numerically.

If the first order phonon-assisted densities in the differential equation for the interband polarization are replaced by the expressions which appear on the right-hand sides of Equations 2.85 and 2.86, the following integro-differential equation for the components of the interband polarization

$$
\begin{aligned}
i \frac{d}{d t}\left\langle\psi_{v l}^{\dagger} \psi_{c l}\right\rangle_{t} & =E(l, 0)\left\langle\psi_{v l}^{\dagger} \psi_{c l}\right\rangle_{t}-\frac{U}{N} \sum_{k}\left\langle\psi_{v k}^{\dagger} \psi_{c k}\right\rangle_{t}-d E(t) \\
& +\sum_{k} \int_{-\infty}^{\infty} d \tau \bar{S}_{l ; k}(t-\tau)\left\langle\psi_{v k}^{\dagger} \psi_{c k}\right\rangle_{\tau}
\end{aligned}
$$

is obtained in the limit $t_{0} \rightarrow-\infty$ where the memory function $\bar{S}_{l_{1} ; l_{2}}(t)$ satisfies the relation

$$
\begin{aligned}
\bar{S}_{l_{1} ; l_{2}}(t) & =(-i) \frac{g^{2}}{N}\left\{\left\{1+n_{B}\left(\omega_{L O}\right)\right\} e^{-i \omega_{L O} t}+n_{B}\left(\omega_{L O}\right) e^{i \omega_{L O} t}\right\} \theta(t) \\
& \times \sum_{q \neq 0}\left\{G_{l_{1} ; l_{2}}(q ; t)+G_{l_{1}-q ; l_{2}-q}(q ; t)-G_{l_{1}-q ; l_{2}}(q ; t)-G_{l_{1} ; l_{2}-q}(q ; t)\right\} .
\end{aligned}
$$

The first three terms on the right-hand side of Equation 2.96 describe the undamped dynamics of an optically excited electron-hole pair with a vanishing center-of-mass 
momentum while the last term is responsible for the decay of the excited state due to phonon emission and absorption processes.

The expression in Equation 2.96 could be used as a starting point for a detailed numerical calculation of the linear response of the semiconductor. However, since the interest is mainly focused on the effect of the vertex corrections, the integro-differential equation for the interband polarization is now solved analytically by neglecting all contributions to the polarization which come from the continuum states. Then the dynamics of the components of the interband polarization only depends on the time behavior of the excitonic state

$$
\left\langle\psi_{v l}^{\dagger} \psi_{c l}\right\rangle_{t}=\Phi_{l}^{e x} P_{e x}(t)
$$

where the definition for the vector components of the excitonic eigenstate can be found in Appendix B. By means of Equation 2.96 the following identity for the excitonic polarization

$$
P_{e x}(t)=d \sum_{k} \Phi_{k}^{e x} \int_{-\infty}^{t} d \tau \bar{\chi}_{e x}(t-\tau) E(\tau)
$$

can be derived. The excitonic susceptibility $\bar{\chi}_{e x}(t)$ satisfies the differential equation

$$
i \frac{d}{d t} \bar{\chi}_{e x}(t)=\omega_{e x}(0) \bar{\chi}_{e x}(t)+\int_{0}^{\infty} d \tau \bar{S}_{e x}(t-\tau) \bar{\chi}_{e x}(\tau)
$$

with the initial condition $\bar{\chi}_{e x}(0)=-1$. The explicit expression for the energy of the exciton with no center-of-mass momentum $\omega_{e x}(0)$ can be found in Appendix B. The function $\bar{S}_{e x}(t)$ is the diagonal element of the matrix $\bar{S}_{l_{1} ; l_{2}}(t)$ with respect to the excitonic state. It is given by

$$
\begin{aligned}
\bar{S}_{e x}(t) & =(-i) \frac{g^{2}}{N}\left\{\left\{1+n_{B}\left(\omega_{L O}\right)\right\} e^{-i \omega_{L O} t}+n_{B}\left(\omega_{L O}\right) e^{i \omega_{L O} t}\right\} \theta(t) \\
& \times \sum_{q \neq 0} \sum_{k_{1} k_{2}}\left\{\Phi_{k_{1}+q}^{e x}-\Phi_{k_{1}}^{e x}\right\}\left\{\Phi_{k_{2}+q}^{e x}-\Phi_{k_{2}}^{e x}\right\} G_{k_{1} ; k_{2}}(q ; t) .
\end{aligned}
$$

The integro-differential equation for the susceptibility $\bar{\chi}_{e x}(t)$ can be solved easily with the help of a Fourier transformation:

$$
\chi_{e x}(\omega)=\int_{0}^{\infty} d \tau e^{i \omega t} \bar{\chi}_{e x}(t)=-\frac{1}{\omega+i 0-\omega_{e x}(0)-S_{e x}(\omega+i 0)} .
$$

If the electrons and phonons did not interact with each other, the denominator in Equation 2.102 would simply describe a resonance at the exciton energy $\omega_{e x}(0)$. The influence of the electron-phonon scattering processes is taken into account by the self- 
energy function $S_{e x}(\omega+i 0)$ which is defined by the relation

$$
\begin{aligned}
S_{e x}(\omega+i 0)= & \frac{g^{2}}{N} \sum_{q \neq 0} \sum_{k_{1} k_{2}}\left\{\Phi_{k_{1}+q}^{e x}-\Phi_{k_{1}}^{e x}\right\}\left\{\Phi_{k_{2}+q}^{e x}-\Phi_{k_{2}}^{e x}\right\} \\
& \times\left\{1+n_{B}\left(\omega_{L O}\right)\right\}\left\langle k_{1}, q\left|\hat{G}\left(q ; \omega-\omega_{L O}+i 0\right)\right| k_{2}, q\right\rangle \\
+ & \frac{g^{2}}{N} \sum_{q \neq 0} \sum_{k_{1} k_{2}}\left\{\Phi_{k_{1}+q}^{e x}-\Phi_{k_{1}}^{e x}\right\}\left\{\Phi_{k_{2}+q}^{e x}-\Phi_{k_{2}}^{e x}\right\} \\
& \times n_{B}\left(\omega_{L O}\right)\left\langle k_{1}, q\left|\hat{G}\left(q ; \omega+\omega_{L O}+i 0\right)\right| k_{2}, q\right\rangle .
\end{aligned}
$$

This self-energy function can be split up into the contributions of the phonon emission and the phonon absorption processes. Both contributions have the same form, only their prefactors and their positions in the spectrum with respect to $\omega_{e x}(0)$ are different. As the spectrum of the dark states $\left\langle\psi_{v l+p}^{\dagger} \psi_{c l} b_{p}\right\rangle_{t}$ is located above the exciton energy their influence on the form of $\chi_{e x}(\omega)$ is negligible in comparison with the influence of the dark states $\left\langle\psi_{v l-p}^{\dagger} \psi_{c l} b_{p}^{\dagger}\right\rangle_{t}$.

In Figures 2.5 and 2.6 the function $S_{e x}(\omega)$ has been plotted using the kinetic equations with and without vertex corrections for a system with $N=2000$ elementary cells. The model parameters are those which have already been listed in Section 2.1 and the energy $\omega$ is measured with respect to the renormalized band gap $\tilde{\Delta}=\Delta+U$. The self-energy is only depicted in the vicinity of the energy $\omega_{e x}(0) \approx-0.4 \omega_{L O}$ since the form of $\chi_{e x}(\omega)$ is mainly determined by the behavior of the denominator from Equation 2.102 near the exciton energy. If the self-energy is calculated with the vertex corrections, its imaginary part exhibits a sharp peak at the energy $\omega \approx-0.9 \omega_{L O}$. This peak denotes the position of the upper edge of the excitonic band for the dark states which are coupled to the optically excited exciton by phonon absorption processes. For energies which are smaller than the energy of the lower edge of this excitonic band $\left(\omega \approx-1.4 \omega_{L O}\right)$ the imaginary part vanishes because there exist no dark states with a smaller energy. If the vertex corrections are not taken into account, this lower threshold for the imaginary part is shifted to $\omega=-\omega_{L O}$ since the spectrum of the dark states $\left\langle\psi_{v l-p}^{\dagger} \psi_{c l} b_{p}^{\dagger}\right\rangle_{t}$ is now similar to the spectrum of a free electron-hole pair with no excitonic resonances. The peak at $\omega \approx-0.4 \omega_{L O}$ is due to the upper band edge of the valence band. It is conspicuous that the singularity at the lower edge of the excitonic band is not visible in Figure 2.5. This is due to the fact that the difference $\Phi_{k+q}^{e x}-\Phi_{k}^{e x}$ which appears as a factor in the two sums of Equation 2.103 vanishes continuously in the limit $q \rightarrow 0$. Consequently, the contributions of the excitonic states with eigenenergies $\omega_{x}(q)$ near the lower band edge $(q=0)$ are suppressed. A similar explanation can be used in order to understand the missing singularity at $\omega=-\omega_{L O}$ in Figure 2.6 where the lower band edge of the free spectrum of the dark states $\left\langle\psi_{v l-p}^{\dagger} \psi_{c l} b_{p}^{\dagger}\right\rangle_{t}$ is located.

The effect of the self-energy corrections on the form of the susceptibility function $\chi_{e x}(\omega)$ can be studied in Figure 2.7 where its imaginary part is plotted with and without vertex corrections for two different temperatures. For comparison, the imaginary 


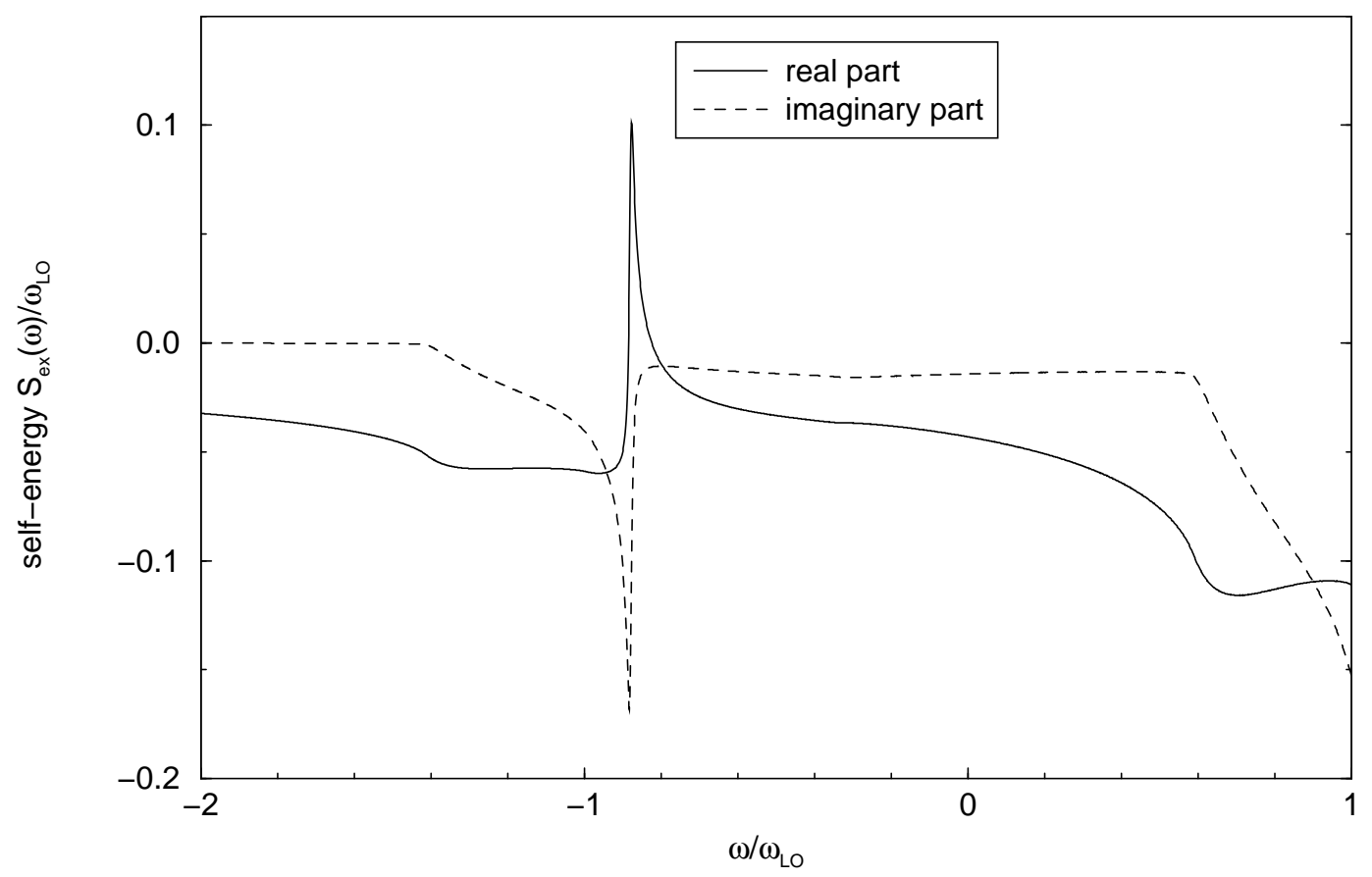

Figure 2.5: The excitonic self-energy $S_{e x}(\omega)$ in the vicinity of $\omega_{e x}(0)$ at the temperature $k_{B} T=0.8 \omega_{L O}$, calculated with vertex corrections

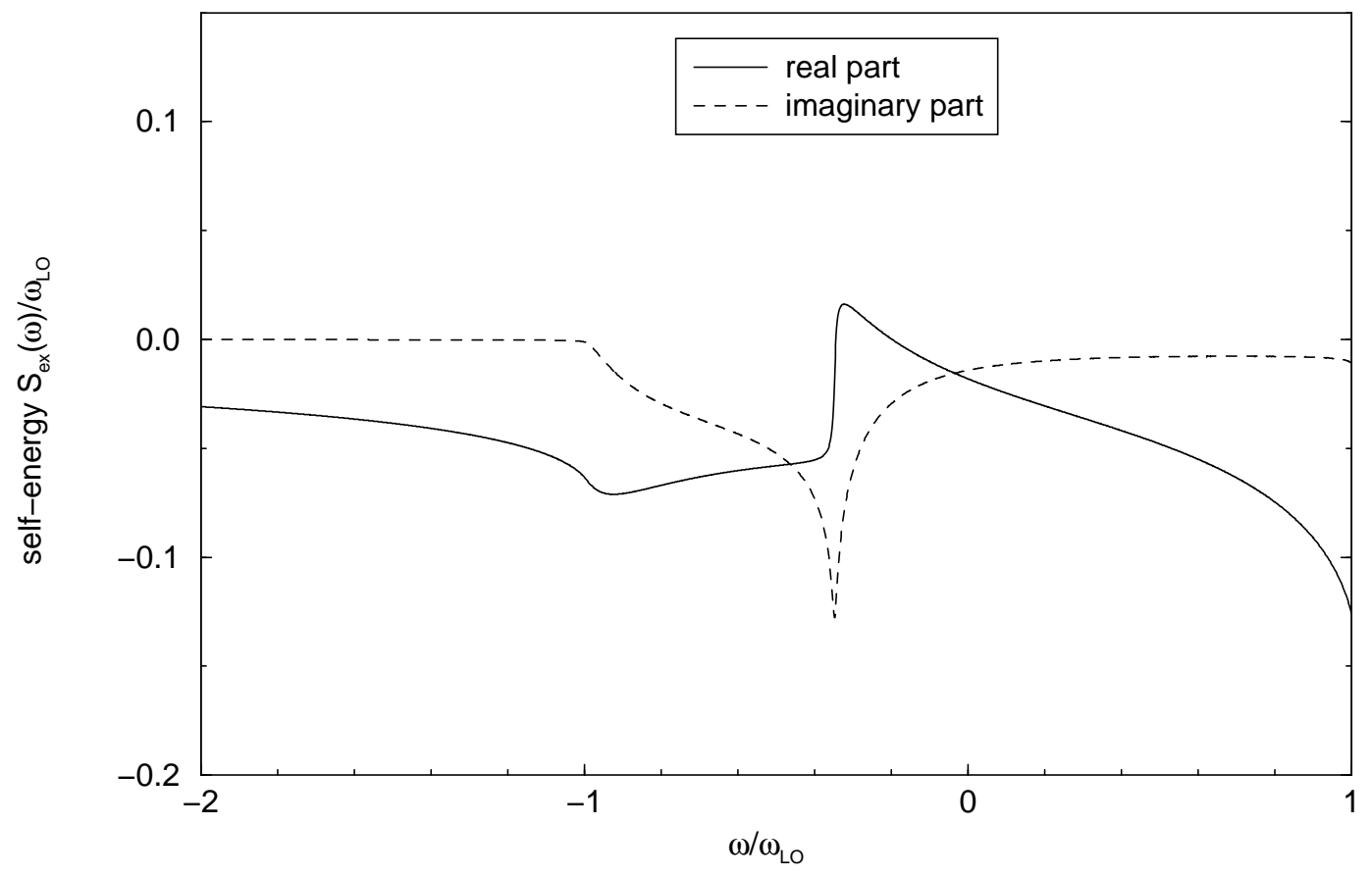

Figure 2.6: The excitonic self-energy $S_{e x}(\omega)$ in the vicinity of $\omega_{e x}(0)$ at the temperature $k_{B} T=0.8 \omega_{L O}$, calculated without vertex corrections 


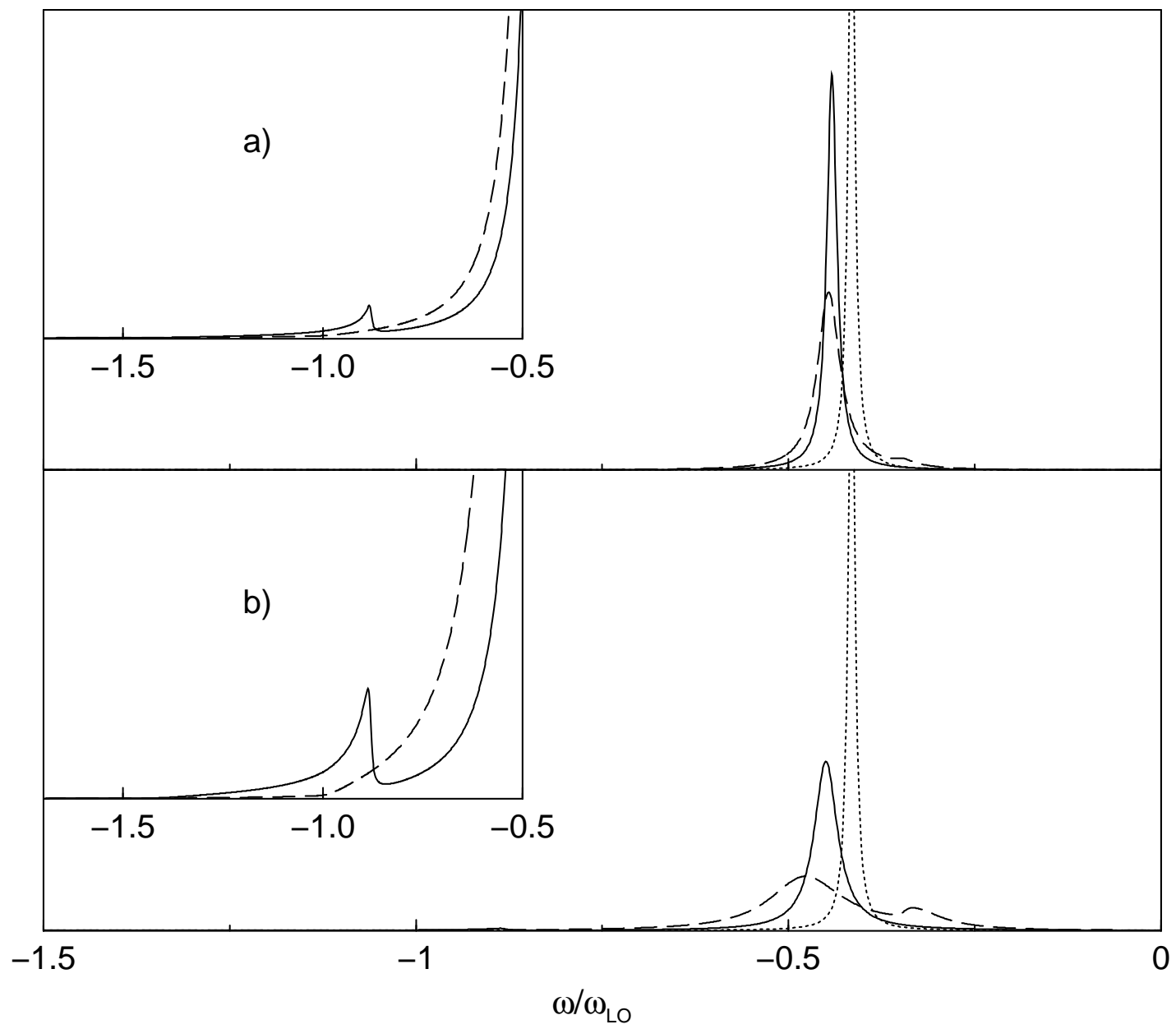

Figure 2.7: The imaginary part of the excitonic susceptibility $\chi_{e x}(\omega)$ at the temperatures a) $k_{B} T=0.4 \omega_{L O}$ and b) $k_{B} T=0.8 \omega_{L O}$, calculated with (solid line) and without (dashed line) vertex corrections, the dotted line denotes the imaginary part of $\chi_{e x}(\omega)$ in the absence of the electron-phonon interaction

part of the susceptibility for a free exciton is also plotted where a small broadening has been added. If no vertex corrections are taken into account, the formation of a double peak structure at the shifted excitonic resonance can be observed with increasing temperature. This is due to the fact that the energy for the exciton with no center-of-mass momentum nearly coincides with the energy of the upper band edge of the valence band in the spectrum for the dark states $\left\langle\psi_{v l-p}^{\dagger} \psi_{c l} b_{p}^{\dagger}\right\rangle_{t}$. If the vertex corrections are included, the exciton peak broadens with increasing temperature, but no splitting can be observed since the real and the imaginary part of the self-energy are only slowly variable in the vicinity of $\omega_{e x}(0)$. The resonance structure which appears below the exciton peak is due to the singularity of the self-energy at the upper edge of the excitonic band of the dark states (confer Figure 2.5).

The explanation for the resonance structure below the excitonic energy is corrob- 
orated, if the imaginary parts of the excitonic susceptibility functions for different electron-hole mass ratios $\kappa$ are compared (Figure 2.8). In agreement with analytical results the upper band edge is shifted towards $\omega=-\omega_{L O}+\omega_{e x}(0)$ for decreasing $\kappa$. If the hole mass is infinite, the excitonic band has no dispersion and only a sharp resonance at $\omega=-\omega_{L O}+\omega_{e x}(0)$ occurs.

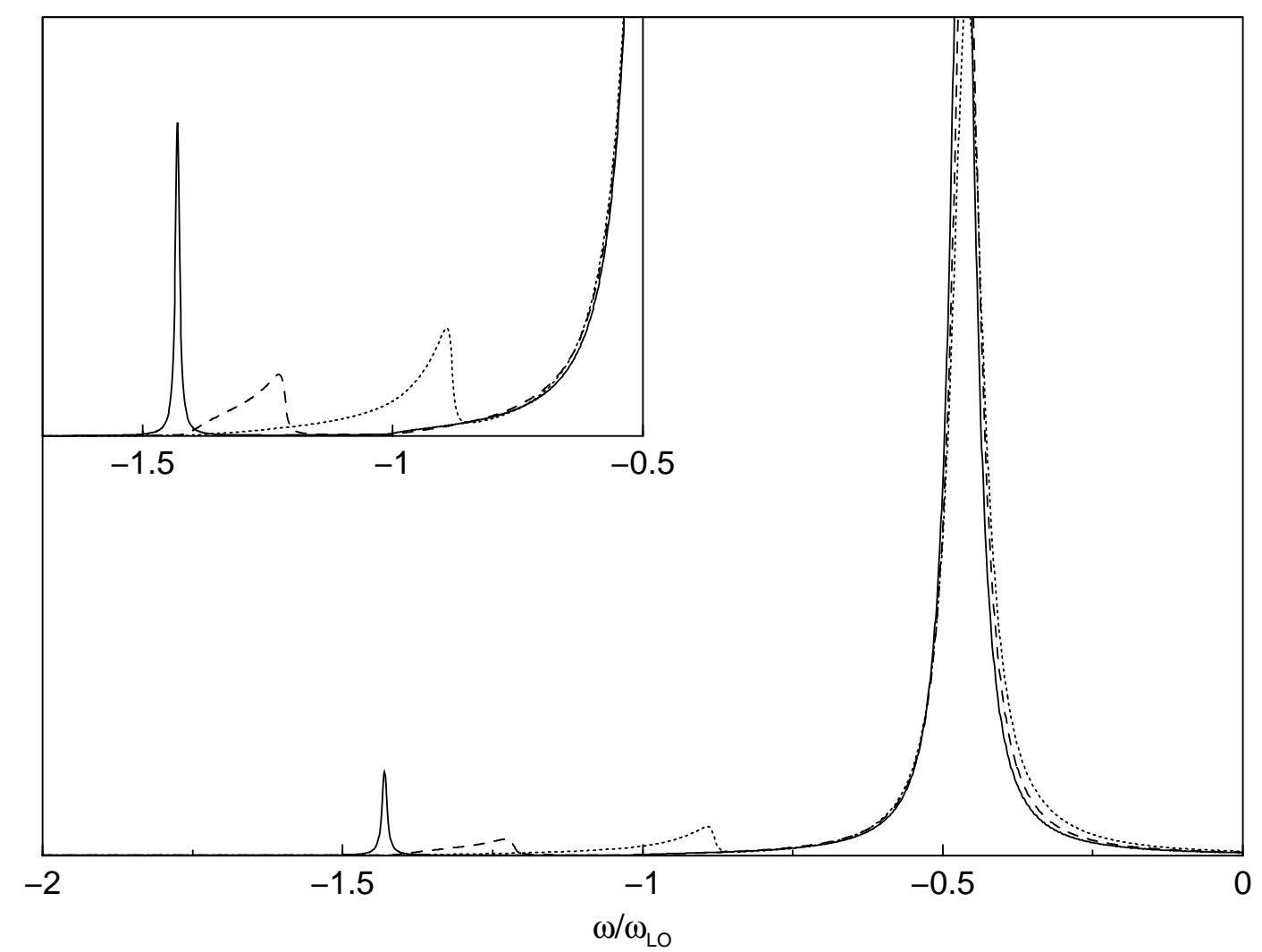

Figure 2.8: The imaginary part of the excitonic susceptibility $\chi_{e x}(\omega)$ at the temperature $k_{B} T=1.2 \omega_{L O}$, calculated with vertex corrections for $\kappa=0.15$ (dotted line), $\kappa=0.05$ (dashed line) and $\kappa=0$ (solid line)

In Reference [56] the imaginary part of the excitonic susceptibility function has been calculated for the same model and the same parameters which have been used here by solving the complete system of linear differential equations numerically with the help of the Lanczos algorithm. The resulting curves are in good agreement with the approximated ones from Figures 2.7 and 2.8 apart from small additional resonance structures below the upper edge of the excitonic band for the dark states $\left\langle\psi_{v l-p}^{\dagger} \psi_{c l} b_{p}^{\dagger}\right\rangle_{t}$. The comparison shows that the qualitative features of the linear excitonic susceptibility are reproduced well within the framework of the diagonal approximation. In general, this is not true [21].

The phonon emission process which is responsible for the relaxation of highly 


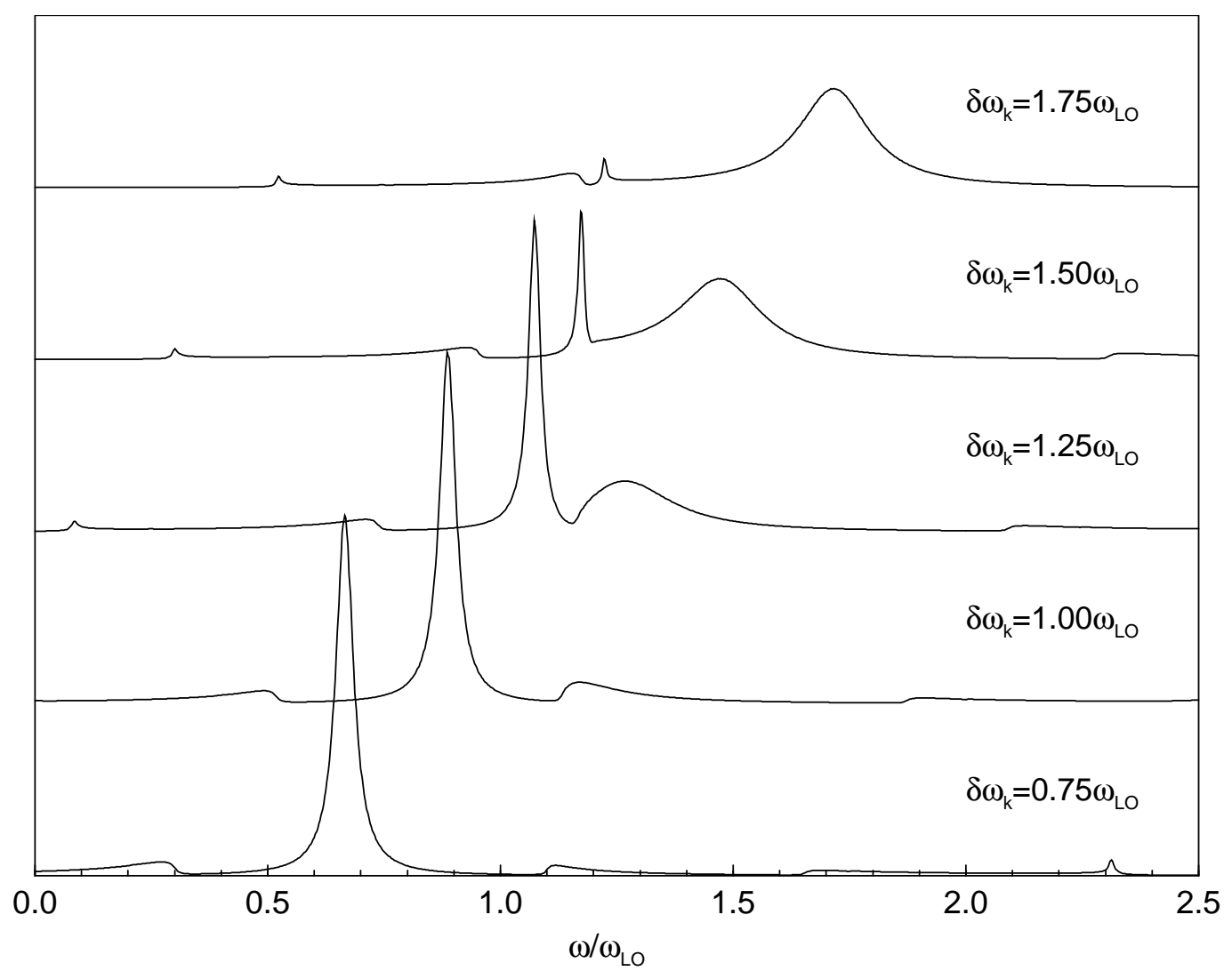

Figure 2.9: The imaginary part of the susceptibility $\chi_{k}(\omega)$ at the temperature $k_{B} T=$ $0.4 \omega_{L O}$ for different continuum energies $\delta \omega_{k}=\omega_{k}-\tilde{\Delta}$

excited electrons can be studied more closely by calculating the susceptibility

$$
\chi_{k}(\omega)=-\frac{1}{\omega+i 0-\omega_{k}-S_{k}(\omega+i 0)}
$$

for a continuum state with energy $\omega_{k}$ (confer Appendix B). The self-energy function $S_{k}$ is defined in the same way as the self-energy for the exciton, only the coefficients for the excitonic eigenvector, $\Phi_{l}^{x}$, are replaced by the corresponding coefficients for the continuum state with energy $\omega_{k}, \Phi_{l}^{k}$. In Figure 2.9 the imaginary part of $\chi_{k}(\omega)$ has been plotted for different energies $\omega_{k}$. If $\omega_{k}$ is smaller than a characteristic threshold energy $\omega_{t}$, the spectral function is peaked sharply at $\omega_{k}$. This corresponds to a slow decay of the initial state. For energies $\omega_{k}$ which are in the vicinity of $\omega_{t}$ one observes a double peak structure which is due to the strong coupling between electron and phonon modes. For $\omega_{k} \gg \omega_{t}$ the spectrum exhibits a broad Lorentzian peak at $\omega_{k}$ indicating a fast decay of the initial state. The existence of this threshold energy shows that the life-time of the continuum states is mainly determined by the influence of the phonon emission processes. A fast decay of excited continuum states can only be observed, if a 
scattering into continuum states with a lower energy by phonon emission is consistent with the classical energy conservation. For this reason, one would expect $\omega_{t}$ to be equal to $\omega=\omega_{L O}+\Delta+U$. The observed threshold energy, however, is located above this energy value. This discrepancy can be explained by the fact that $\chi_{k}(\omega)$ is the spectral function for the electron-hole pairs. Consequently, the frequency $\omega$ refers to the pair energy and not to the energy of the conduction electrons alone.

The phenomenon of the shifted threshold energy can be explained analytically by calculating the susceptibility $\chi_{k}(\omega)$ for non-interacting electrons $(U=0$ and $\tilde{U}=0)$ at zero temperature. Since the external field only excites electron-hole pairs with even parity the components of the eigenvector which describes the optically excited state with energy $\omega_{k}=E(k, 0)$ are defined by

$$
\Phi_{l}^{k}=\frac{1}{\sqrt{2}}\left\{\delta_{l ; k}+\delta_{l ;-k}\right\} .
$$

If the self-energy function is evaluated at the resonance $\omega=\omega_{k}$, the susceptibility is approximately given by

$$
\chi_{k}(\omega) \approx-\frac{1}{\omega+i 0-\omega_{k}-S_{k}\left(\omega_{k}+i 0\right)} .
$$

In the thermodynamic limit $S_{k}\left(\omega_{k}+i 0\right)$ satisfies the equation

$$
\begin{aligned}
S_{k}\left(\omega_{k}+i 0\right) & =\frac{g^{2}}{2 \pi} \int_{-\pi}^{\pi} d q \frac{1}{\omega_{k}+i 0-\omega_{L O}-\epsilon_{c ; k+q}+\epsilon_{v ; k}} \\
& +\frac{g^{2}}{2 \pi} \int_{-\pi}^{\pi} d q \frac{1}{\omega_{k}+i 0-\omega_{L O}-\epsilon_{c ; k}+\epsilon_{v ; k+q}}
\end{aligned}
$$

and the imaginary part of the self-energy is only different from zero, if one of the two following conditions

$$
\begin{aligned}
4 t_{c}+\Delta+\omega_{L O} & \geq \epsilon_{c ; k} \geq \Delta+\omega_{L O} \\
\Leftrightarrow 4\left\{t_{c}+t_{v}\right\}+\Delta+\omega_{L O}\{1+\kappa\} & \geq \omega_{k} \geq \Delta+\omega_{L O}\{1+\kappa\}
\end{aligned}
$$

and

$$
\begin{aligned}
4 t_{v}+\omega_{L O} & \geq-\epsilon_{v ; k} \geq \omega_{L O} \\
\Leftrightarrow 4\left\{t_{c}+t_{v}\right\}+\Delta+\omega_{L O}\left\{1+\kappa^{-1}\right\} & \geq \omega_{k} \geq \Delta+\omega_{L O}\left\{1+\kappa^{-1}\right\}
\end{aligned}
$$

is met. If the parameters from Section 2.1 are used, only the first relation can be satisfied and the threshold energy is equal to $\omega_{t}=\omega_{L O}\{1+\kappa\}$ in agreement with the experimental results of Reference [39]. 


\subsection{Nonlinear Response}

If the full nonlinear quantum kinetic equations are used to describe the response of the system to an optical pulse, all electron and phonon densities have to be calculated explicitly in contrast to the treatment within the framework of the linear response theory where only the off-diagonal elements of the density matrix show a nontrivial time behavior. After electron-hole pairs with zero center-of-mass momentum have been created by the laser pulse the interaction of the electron-hole pairs with the lattice leads to the creation and absorption of phonons with energy $\omega_{L O}$ and nonzero center-of-mass momentum electron-hole pairs. These processes are reflected in the time behavior of the distribution functions for the electrons and the phonons.

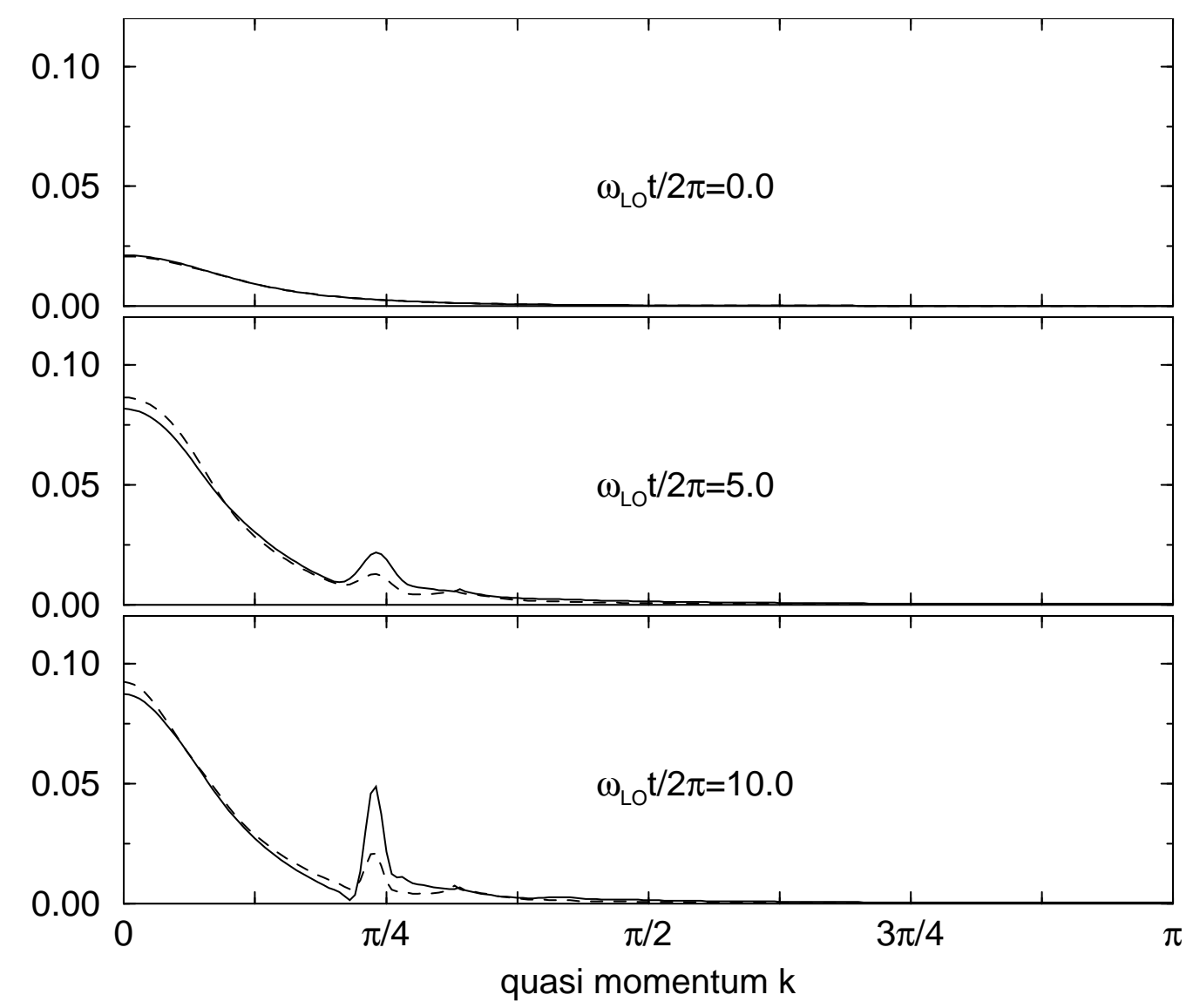

Figure 2.10: The electron distribution $\left\langle\psi_{c k}^{\dagger} \psi_{c k}\right\rangle_{t}$ for $\delta \omega_{P}=-0.4 \omega_{L O}$, calculated with (solid line) and without (dashed line) vertex corrections

When calculating the dynamics of the semiconductor it is assumed that the electric field $E(t)$ is given by a pulse with a Gaussian profile centered at $t=0$ :

$$
E(t)=\mathcal{E}(t) \cos \left(\omega_{P} t\right)=\frac{2 \sqrt{\pi}}{\sigma} \tilde{A} e^{-\frac{t^{2}}{\sigma^{2}}} \cos \left(\omega_{P} t\right) .
$$




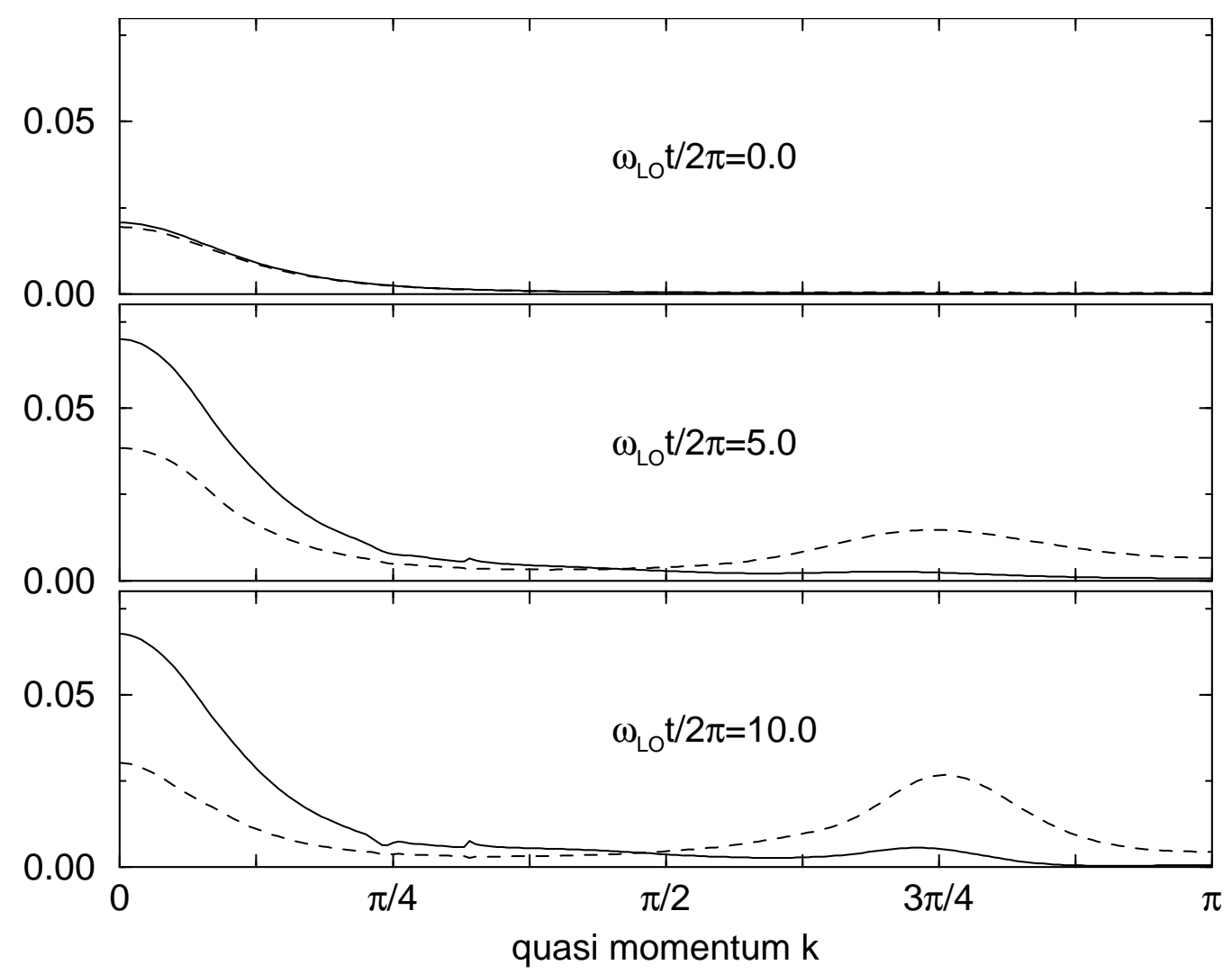

Figure 2.11: The hole distribution $\left\langle\psi_{v k} \psi_{v k}^{\dagger}\right\rangle_{t}$ for $\delta \omega_{P}=-0.4 \omega_{L O}$, calculated with (solid line) and without (dashed line) vertex corrections

As far as the interaction of the external field with the semiconductor is concerned, only the resonant terms of $E(t)$ are taken into account (rotating wave approximation). The Gaussian pulse $\mathcal{E}(t)$ has a full width at half maximum of $\Delta_{\omega}=0.3 \omega_{L O}$ in the frequency representation which corresponds to a full width at half maximum of $\Delta_{t}=$ $5.3 \pi \omega_{L O}^{-1}=2 \sqrt{\ln 2} \sigma$ in the time representation. The strength of the pulse is chosen such that the time integral for $d \mathcal{E}(t)$ gives $5 \%$ of a $2 \pi$ pulse which means that the dynamics is studied in the weak nonlinear excitation regime. In order to study the effect of the vertex corrections, the nonlinear dynamics of the system is investigated for different detunings $\delta \omega_{P}=\omega_{P}-\tilde{\Delta}$ with respect to the renormalized band gap $\tilde{\Delta}=\Delta+U$ whose value is only affected by the on-site component of the electronelectron interaction while a possible long-range part has no impact of the shift of the band gap. The quantum kinetic equations are solved using an explicit Runge-Kutta method of order four. The number of sites is set equal to $N=450$. Concerning the lattice temperature it is assumed that $k_{B} T=0.4 \omega_{L O}$.

If the excitation is tuned to the excitonic resonance $\left(\delta \omega_{P}=-0.4 \omega_{L O} \approx \omega_{e x}(0)-\right.$ $\tilde{\Delta})$, the formation of the exciton can be recognized in the distribution functions for the conduction electrons $\left\langle\psi_{c k}^{\dagger} \psi_{c k}\right\rangle_{t}$ in Figure 2.10 and for the valence holes $\left\langle\psi_{v k} \psi_{v k}^{\dagger}\right\rangle_{t}$ 


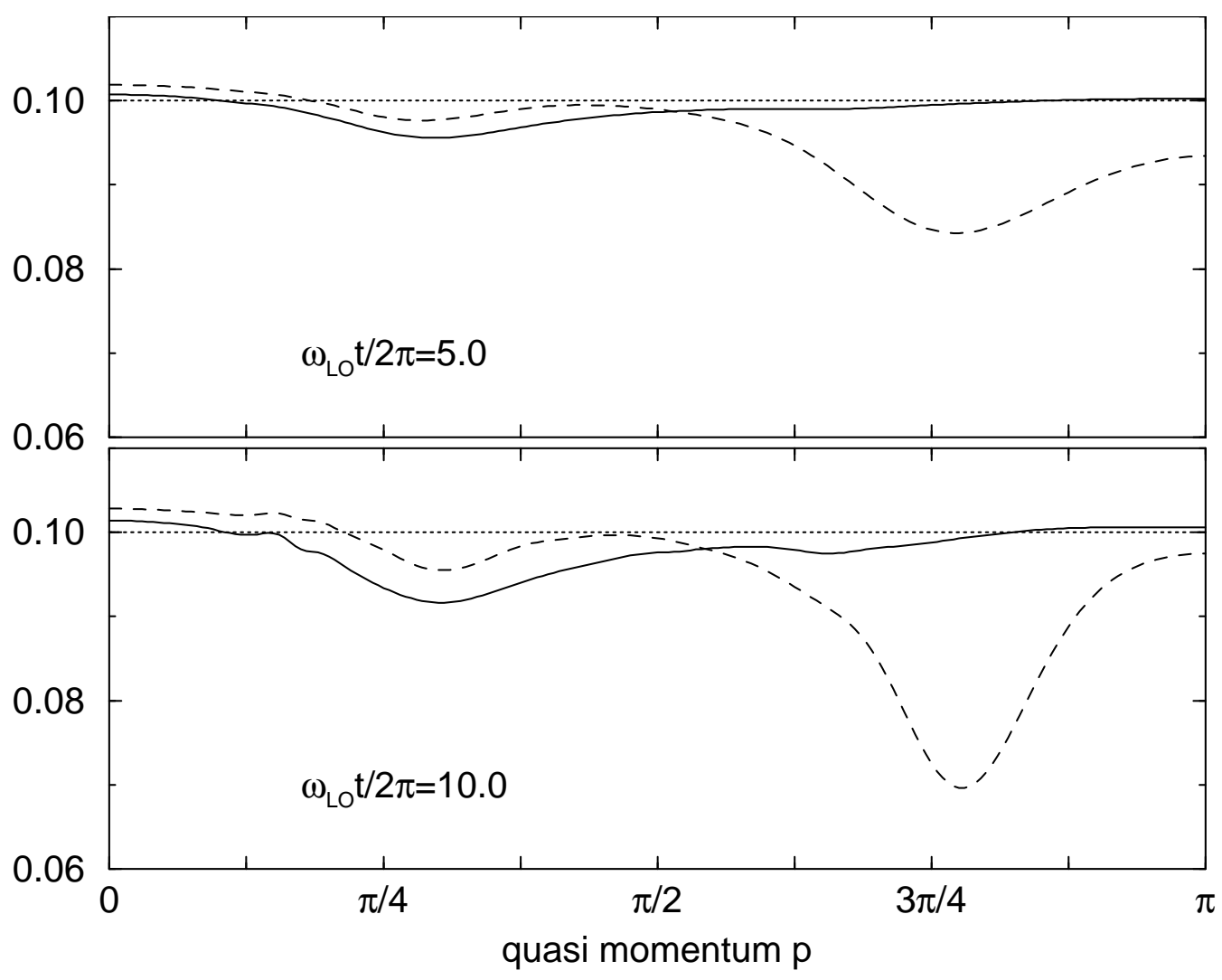

Figure 2.12: The phonon distribution $\left\langle b_{p}^{\dagger} b_{p}\right\rangle_{t}$ for $\delta \omega_{P}=-0.4 \omega_{L O}$, calculated with (solid line) and without (dashed line) vertex corrections, the dotted line represents the equilibrium distribution before the excitation

in Figure 2.11. Due to the presence of phonons in the initial state the occurrence of an absorption peak near $k=\frac{1}{4} \pi$ for $\left\langle\psi_{c k}^{\dagger} \psi_{c k}\right\rangle_{t}$ and near $k=\frac{3}{4} \pi$ for $\left\langle\psi_{v k} \psi_{v k}^{\dagger}\right\rangle_{t}$ can be noticed. They result from the transition of optically excited bound electronhole pairs with the energy $\omega_{i}=\omega_{e x}(0)$ to scattering states with the energy $\omega_{f}=$ $\omega_{L O}+\omega_{e x}(0)$ which corresponds approximately to the free energy of an electron (hole) with a quasi momentum of $k=\frac{1}{4} \pi\left(k=\frac{3}{4} \pi\right)$. This strongly suggests that the dynamics is dominated by phonon absorption processes in which electrons and holes with a quasi momentum near zero are involved. For larger times it is possible to observe the appearance of additional peaks in the distribution functions for the conduction electrons. They are located above $k=\frac{1}{4} \pi$ and can be interpreted as indicators for multi-phonon absorption processes. All absorption processes are also reflected in the phonon distribution function $\left\langle b_{p}^{\dagger} b_{p}\right\rangle_{t}$. As it can be seen in Figure 2.12, the number of phonons decreases in the vicinity of $k=\frac{1}{4} \pi$ and $k=\frac{3}{4} \pi$ in time. If the vertex corrections are taken into account, the absorption peak of the exciton in the electron distribution is more pronounced while the corresponding peak in the hole distribution is almost entirely suppressed. These observations indicate the strong influence of the 
vertex corrections on the time behavior of bound electron-hole pairs in accordance with the results for the linear response in Section 2.5

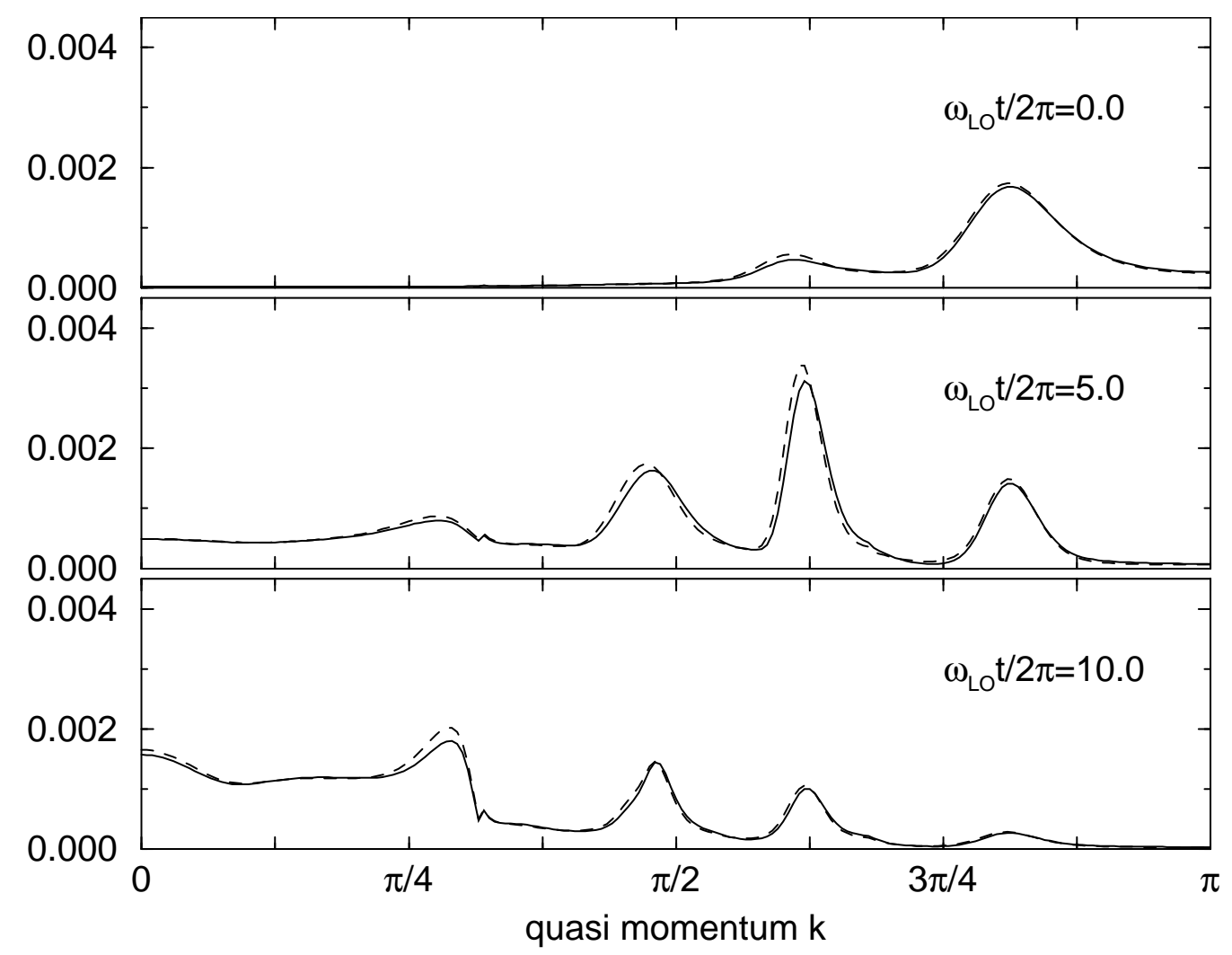

Figure 2.13: The electron distribution $\left\langle\psi_{c k}^{\dagger} \psi_{c k}\right\rangle_{t}$ for $\delta \omega_{P}=4.5 \omega_{L O}$, calculated with (solid line) and without (dashed line) vertex corrections

If continuum states are excited $\left(\delta \omega_{P}>0\right)$, two different scenarios can be observed. In Figure 2.13 the conduction electron distribution is plotted for an excitation far above the band gap $\left(\delta \omega_{P}=4.5 \omega_{L O}\right)$. It is possible to recognize the successive formation of three phonon replicas below the excitation peak which is located above $k \cong \frac{3}{4} \pi$. The use of the quantum kinetic equations makes it possible to describe the development of these replicas whose width decreases with increasing time. If the Boltzmann equations were employed for the description of the dynamics, the width of the replicas would be identical with the width of the original excitation peak [57]. In contrast to the electron distribution, the hole distribution (not plotted here) shows no replica structures. This indicates that only the scattering of phonons and conduction electrons is responsible for the decay of the excited state. The relaxation of holes by phonon emission processes is suppressed since the width of the valence band $4 t_{v}=\kappa 4 t_{c} \approx 0.7 \omega_{L O}$ is smaller than the phonon energy. Therefore the relaxation process for excitations far above the band gap can be described well by using a one-band model $[40,48]$.

If $\delta \omega_{P}$ is chosen to be smaller than $\omega_{L O}$, the excited electron-hole pairs cannot scat- 


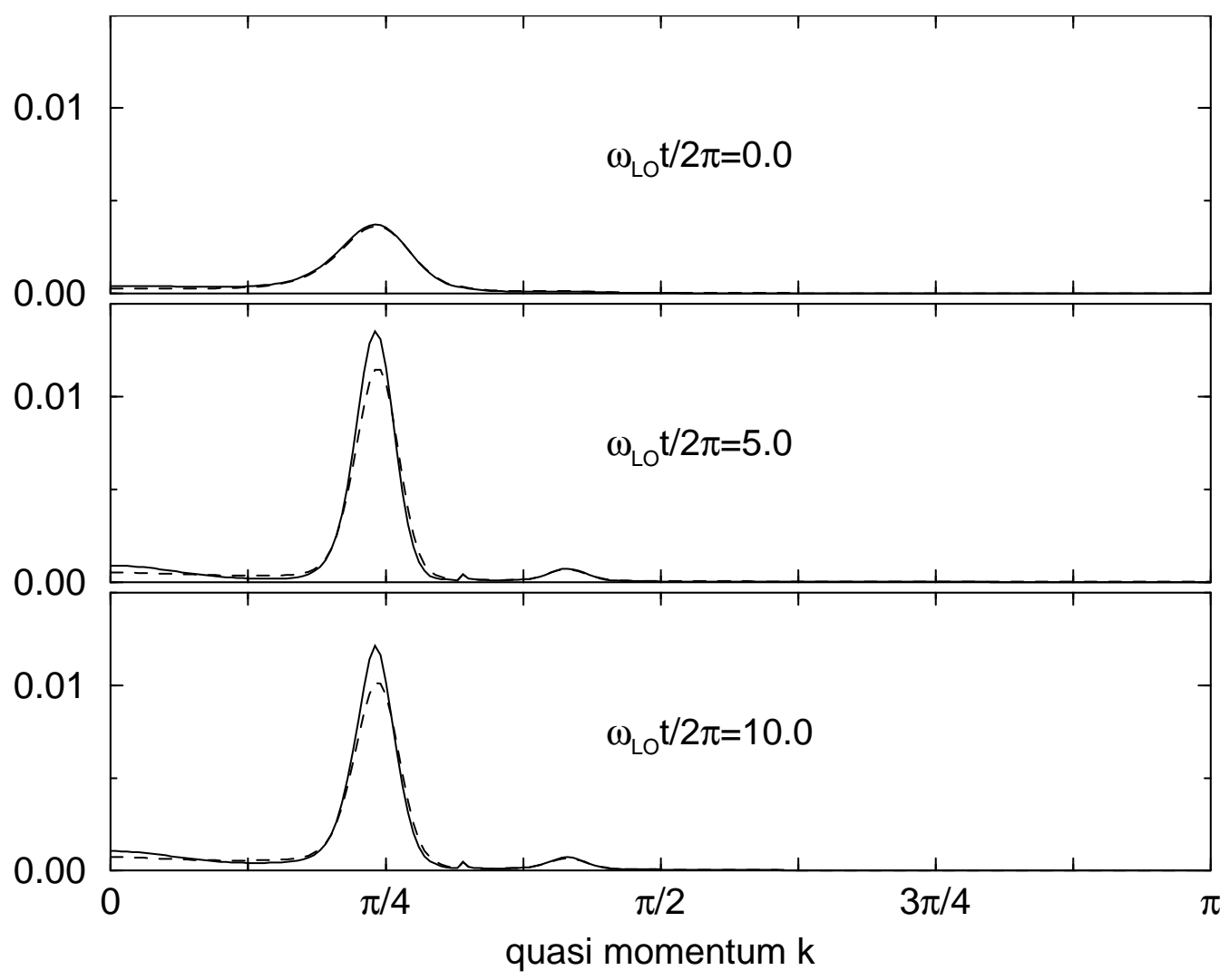

Figure 2.14: The electron distribution $\left\langle\psi_{c k}^{\dagger} \psi_{c k}\right\rangle_{t}$ for $\delta \omega_{P}=0.6 \omega_{L O}$, calculated with (solid line) and without (dashed line) vertex corrections

ter into continuum states with lower energy by phonon emission and the dynamics of the system changes significantly. As an example, the conduction electron distribution for the detuning $\delta \omega_{P}=0.6 \omega_{L O} \approx \omega_{L O}+\omega_{e x}(0)-\tilde{\Delta}$ is plotted in Figure 2.14. After the pulse has been turned off the electron distribution $\left\langle\psi_{c k}^{\dagger} \psi_{c k}\right\rangle_{t}$ changes only slowly. Although a transition of the unbound electron-hole pairs to excitonic states with a finite center-of-mass momentum is allowed according to the rules of classical energy conservation, the formation of an exciton distribution cannot be observed. The only feature which indicates the presence of phonons is the small gain peak above $k \cong \frac{1}{4} \pi$.

The vertex corrections have no significant influence on the dynamics for continuum excitation. This can be explained by the fact that the continuum states for interacting electron-hole pairs differ only slightly from free electron-hole states. Therefore the vertex corrections do not lead to qualitative changes of the time behavior and can be neglected when calculating the optical response in the weak nonlinear regime. In this context it should be mentioned that even the Hartree-Fock corrections are often neglected entirely when describing the relaxation of highly excited electron-hole pairs since the qualitative features can already be understood well within the framework of the free-electron picture [43]. 


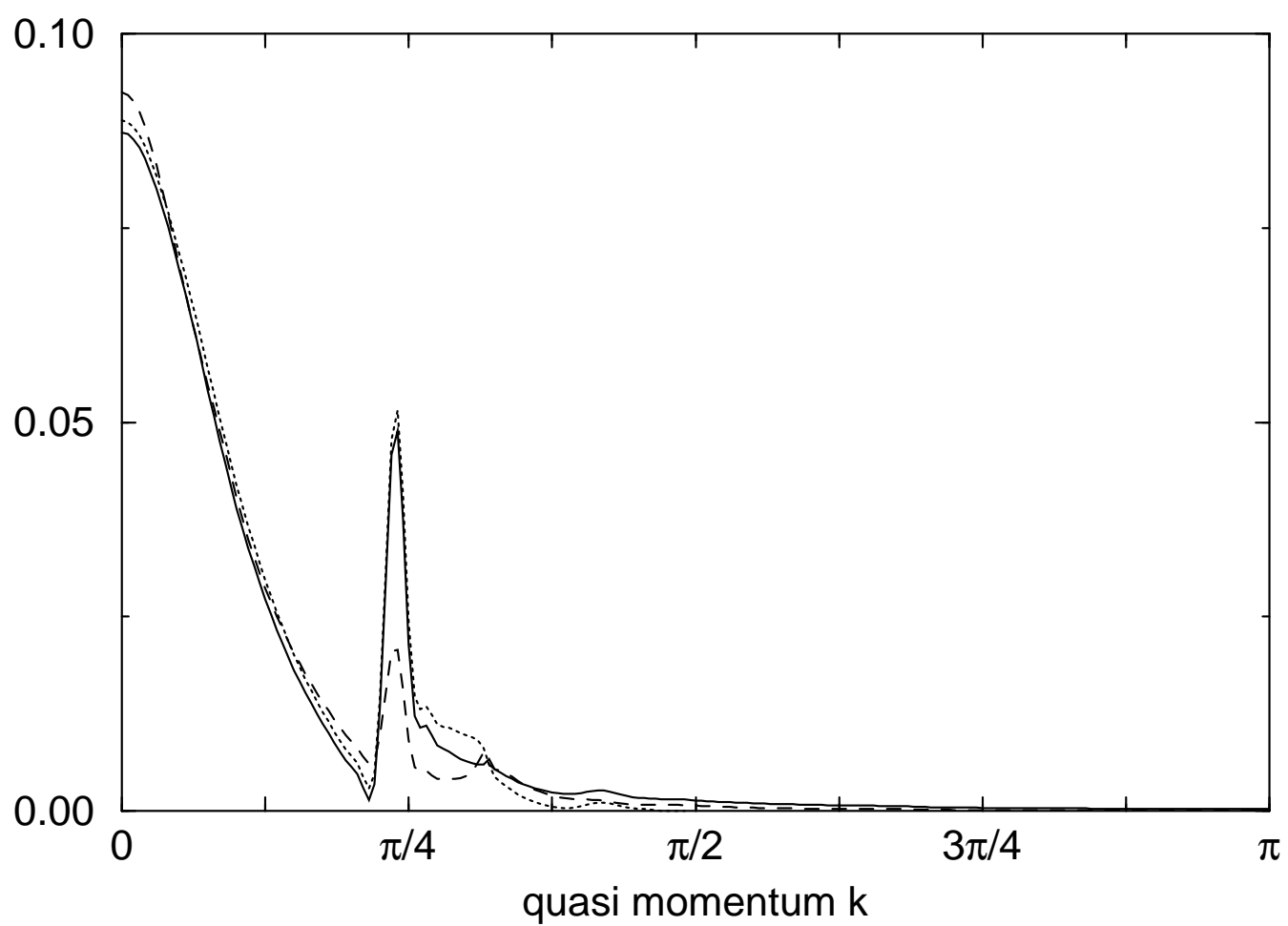

Figure 2.15: The electron distribution $\left\langle\psi_{c k}^{\dagger} \psi_{c k}\right\rangle_{t}$ for $\delta \omega_{P}=-0.4 \omega_{L O}$ and $\omega_{L O} t=$ $20 \pi$, calculated with the complete vertex corrections (solid line), the approximated vertex corrections (dotted line) and without vertex corrections (dashed line)

The correction terms in the first four lines of Equation 2.24 contain sums over the quasi momenta in the first Brillouin zone which have to be performed separately for each phonon-assisted density $\left\langle\psi_{\lambda_{1} l+p}^{\dagger} \psi_{\lambda_{2} l} b_{p}\right\rangle_{t}$. The Hartree-Fock contributions, however, are only calculated once for each time $t$. Consequently, if the nonlinear kinetic equations with the vertex corrections are used for the description of the dynamics, the required computation time is considerably larger than the computation time which is needed, if the vertex corrections are neglected. Since the numerical calculations in this section are restricted to the weak nonlinear regime it seems suggestive to use a simplified version of the vertex corrections instead of the complete ones in order to reduce the computation time. In the following, the vertex corrections are therefore approximated by the expression

$$
\delta\left\langle\psi_{\lambda_{1} l+p}^{\dagger} \psi_{\lambda_{2} l} b_{p}\right\rangle_{e e}=\sum_{k}\left\{\delta_{\lambda_{2} ; v}-\delta_{\lambda_{1} ; v}\right\} V_{\lambda_{1} \lambda_{2}}(|k-l|)\left\langle\psi_{\lambda_{1} k+p}^{\dagger} \psi_{\lambda_{2} k} b_{p}\right\rangle_{t}
$$

where the electronic densities which appear as factors in the vertex corrections have been replaced by their initial values. If the new simplified kinetic equations were linearized with respect to the external field $E(t)$, the equations-of-motion for the interband polarization and the off-diagonal elements of the phonon-assisted density matri- 


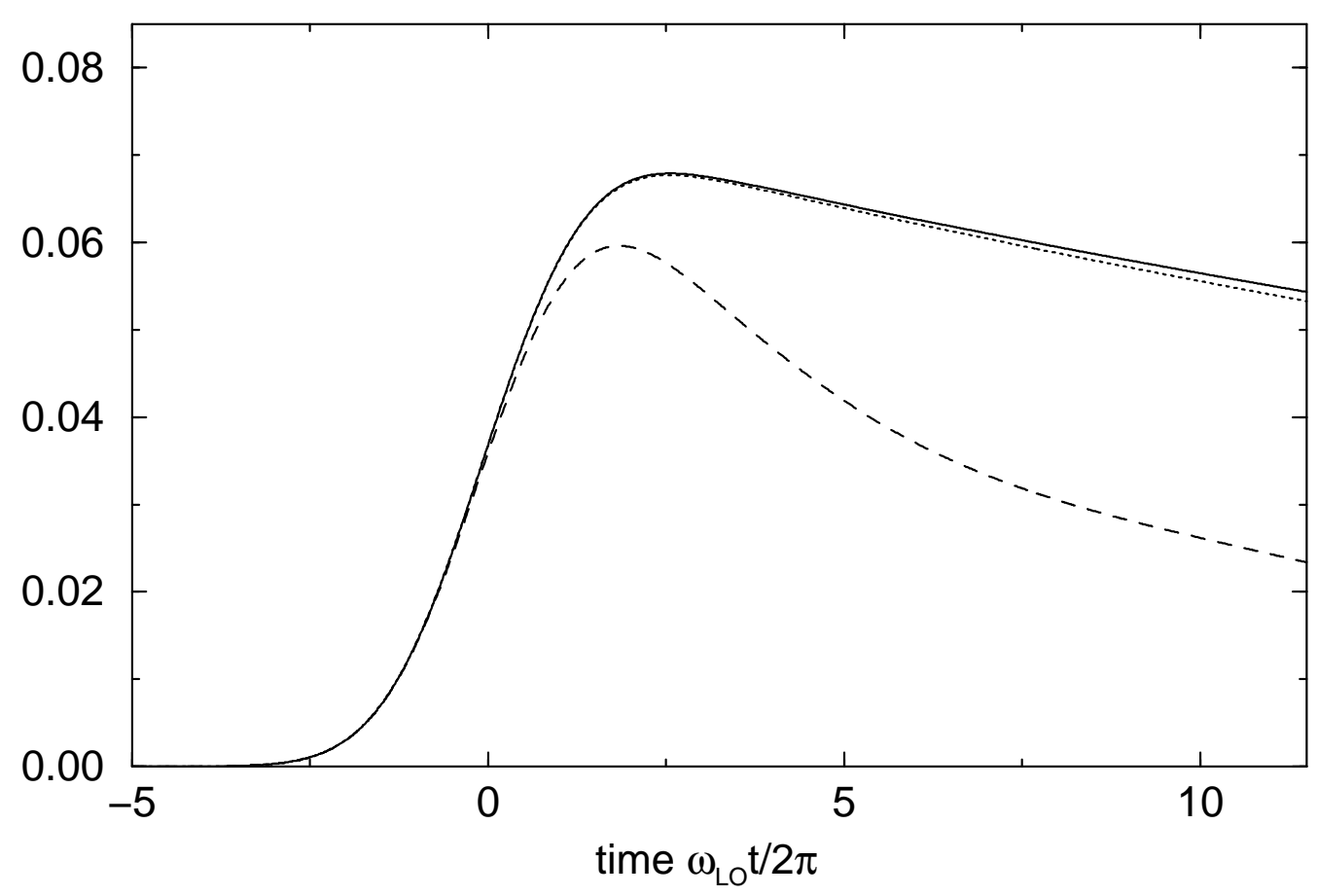

Figure 2.16: The absolute value of the interband polarization $N^{-1} \sum_{k}\left\langle\psi_{v k}^{\dagger} \psi_{c k}\right\rangle_{t}$ for $\delta \omega_{P}=-0.4 \omega_{L O}$, calculated with the complete vertex corrections (solid line), the approximated vertex corrections (dotted line) and without vertex corrections (dashed line)

ces would be equal to the corresponding differential equations from Section 2.5.

Figure 2.15 shows an enlarged version of the lowest graphic from Figure 2.10 where the distribution function which is calculated with the approximated vertex corrections from Equation 2.111 has been added in order to find out if the discrepancies between the two different systems of kinetic equations which have been used for the calculation of the curves in Figures 2.10 to 2.14 are mainly due to the different description of the dynamics of electron-hole pairs with a non-vanishing center-of-mass momentum as in Section 2.5. A comparison of the three curves in Figure 2.15 shows that the absorption peak at $k=\frac{1}{4} \pi$ which belongs to the electron distribution which is calculated with the approximated vertex corrections is in good agreement with the corresponding absorption peak of the electron distribution which is calculated with the help of the complete vertex corrections. However, differences between the two distributions can be observed in the region above this absorption peak. If the approximated vertex corrections are used in the numerical calculations, the occupation numbers for electrons whose kinetic energy is larger than the energy of the conduction electron with the quasi momentum $k=\frac{1}{4} \pi$ and smaller than the threshold energy for absorption pro- 


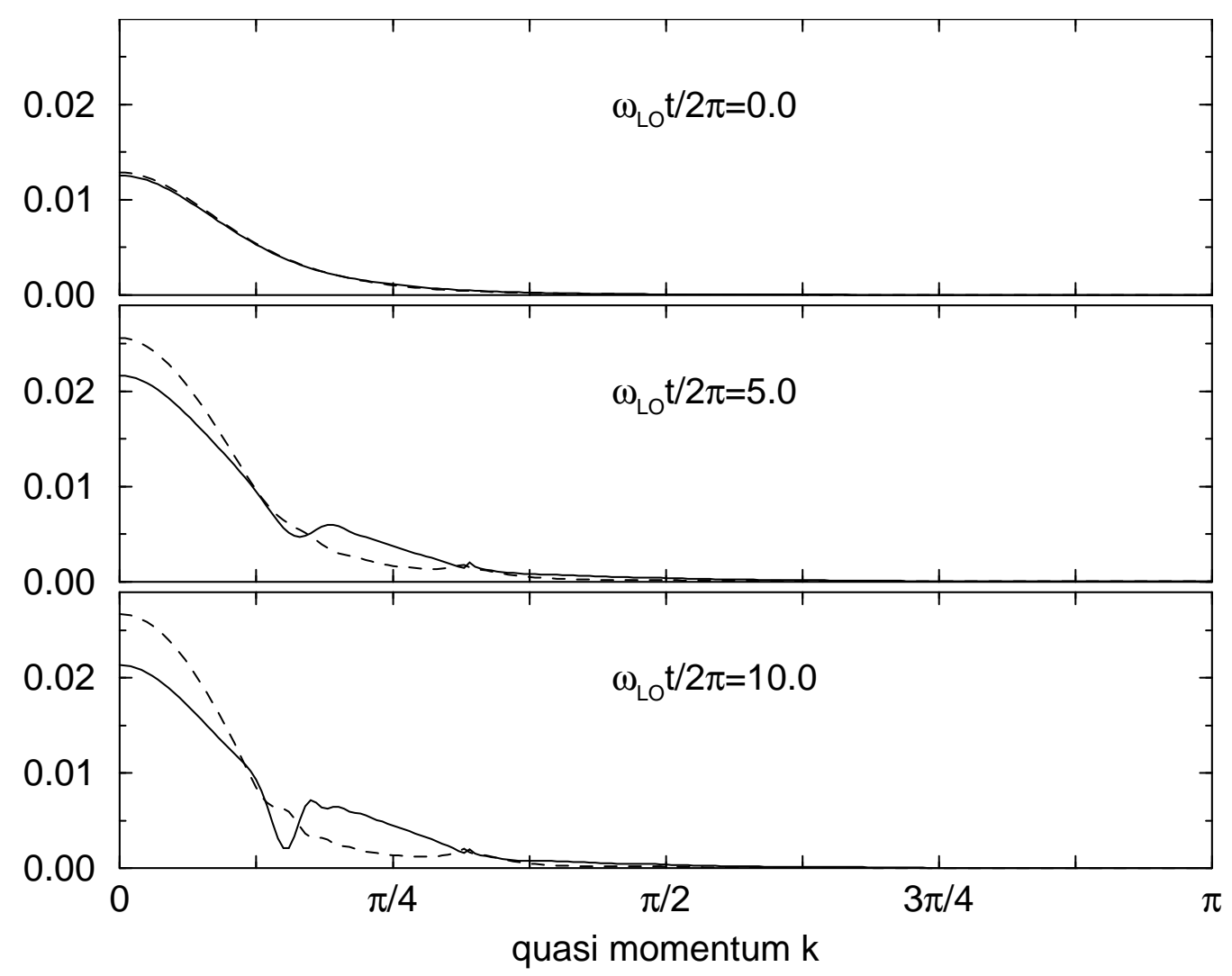

Figure 2.17: The electron distribution $\left\langle\psi_{c k}^{\dagger} \psi_{c k}\right\rangle_{t}$ for $\delta \omega_{P}=-0.6 \omega_{L O}$ in the presence of a long-range Coulomb interaction $2 \tilde{U}=U$, calculated with (solid line) and without (dashed line) vertex corrections

cesses in the continuum band $\left(\omega \approx \omega_{L O}\right)$ are larger than the corresponding occupation numbers of the electron distribution which has been calculated by using the complete vertex corrections. For conduction electrons whose kinetic energy is larger than the above-mentioned threshold energy this relation is reversed. The different behavior of the two curves clearly shows the influence of the nonlinear terms in the complete kinetic equations. A convincing explanation for the differences is still lacking and would certainly require a closer analysis of the specific impact of each individual term of the vertex corrections.

The differences between the three systems of kinetic equations are also reflected in the dynamics of extensive quantities such as the interband polarization of the probe. The curves in Figure 2.16 depict the absolute value of the polarization which has been calculated with the complete and the approximated vertex corrections and without vertex corrections. It turns out that the polarization signal which has been calculated without considering the vertex corrections decays much faster than the other polarization signals. This observation is in accordance with the results of the calculation of the imaginary part of the excitonic susceptibility from Figure 2.7 where the curves which 
are calculated without considering the vertex corrections are considerably wider than the curves which are calculated using the vertex corrections. If the curves in Figure 2.16 are subjected to a closer examination, it emerges that the absolute value of the polarization which has been calculated using the quantum kinetic equations without vertex corrections oscillates weakly with a frequency which is considerably smaller than the phonon frequency $\omega_{L O}$. This behavior can be related to the small side peak in the curve in Figure 2.7 which depicts the imaginary part of the corresponding excitonic susceptibility. The polarization signals which are calculated using the complete or the approximated vertex corrections show an almost identical time behavior. This indicates that the dynamics of the two polarization signals is dominated by the linear contributions which coincide exactly.

If the electron-electron interaction contains a long-range part, the principal differences between the descriptions with and without vertex corrections remain the same although the dynamics of a system with a long-range interaction differs considerably from the dynamics of the system which has been studied until now. In Figure 2.17 the distribution of the conduction electrons has been plotted where the parameter of the long-range part of the electron-electron interaction $\tilde{U}$ is half as large as the parameter for the on-site interaction $U$ while the other model parameters are the same as before. The detuning with respect to the band edge $\tilde{\Delta}$ is chosen such that the density of the excited carriers assumes its maximal value $\left(\delta \omega_{P}=-0.6 \omega_{L O}\right)$. It turns out that the differences between the distribution curves are even more pronounced than in Figure 2.10 . 


\section{Chapter 3}

\section{Nonlinear Optical Dynamics of Systems with a Fermi-Edge Singularity}

In this chapter, the properties of the nonlinear optical response of n-doped semiconductors are at the center of interest. The underlying principles are discussed using a one-dimensional two-band model with linear band dispersions. Within the framework of this model the response functions can be calculated exactly, even if the particles interact with each other.

The structure of the chapter will be outlined now. In Section 3.1 the different parts of the Hamiltonian which describes the two-band model are presented and discussed. In Section 3.2 it is shown how the Hamiltonian can be diagonalized in the presence of the electron-hole interaction by introducing a new representation for the elementary excitations of the many-body system with the help of boson operators. All operators which are crucial for the description of the system are then expressed within the framework of the new bosonic representation. In Section 3.3 the optical response of the many-body system to the excitation by an external electromagnetic field is studied within the framework of the time-dependent perturbation theory. The first and third order response functions are presented in their most general form. Section 3.4 contains a detailed investigation of the properties of the linear absorption spectrum. Particular emphasis is put on studying how the interaction processes influence the exponent of the algebraic singularity at the Fermi-edge in the absorption spectrum. In Section 3.5 the signals of a four-wave-mixing experiment with two excitation pulses are calculated for varying time delays between the pulses. It is studied whether the algebraic behavior of the linear absorption spectrum is reflected in the nonlinear signals. In addition to that, the influence of the finite life-time of the excitations on both the time-resolved and the time-integrated four-wave-mixing signals is investigated. In this context, it should be mentioned that the details of the calculation of the nonlinear response functions will be presented separately in Appendix $\mathrm{C}$ because of their complexity. 


\subsection{The Hamiltonian}

The physical system whose optical properties will be studied in this chapter is described by a one-dimensional two-band model with a completely filled valence band and a conduction band whose states are occupied up to a Fermi energy $E_{f}$. This system is excited by one or several light pulses creating pairs of valence holes and conduction electrons.

If the energy of the additionally created conduction electrons is close to $E_{f}$ and if the interaction between the particles does not lead to the transition of conduction electrons into states whose energy is much larger or smaller than $E_{f}$, only the lowenergy excitations of the Fermi sea in the conduction band have a significant influence on the physical properties of the system.

In this case, it is justified to employ the Tomonaga-Luttinger model [1, 6] for the description of the electrons in the conduction band. Within the framework of this model it is assumed that the electron system consists of two sorts of electrons whose energy dispersions are obtained from the energy dispersion of the conduction band electrons by linearizing it with respect to the two Fermi points at $k=-k_{f}$ and $k=k_{f}$. Following Luttinger's approach in his article from 1963 [6], both sorts of electrons are assumed to have an energy spectrum which is not bounded from below. The ground state of the system is then characterized by two infinite Fermi seas (see Figure 3.1) and the free Hamiltonian for the electrons is given by

$$
\begin{aligned}
H_{0}^{e} & =v_{f} \int_{-\frac{L}{2}}^{\frac{L}{2}} d x: \psi_{e r}^{\dagger}(x)\left\{\frac{1}{i} \frac{d}{d x}-k_{f}\right\} \psi_{e r}(x): \\
& +v_{f} \int_{-\frac{L}{2}}^{\frac{L}{2}} d x: \psi_{e l}^{\dagger}(x)\left\{-\frac{1}{i} \frac{d}{d x}-k_{f}\right\} \psi_{e l}(x):
\end{aligned}
$$

where the field operators for the two sorts of electrons are defined by the two equations

$$
\psi_{e r}(x)=\lim _{\alpha \searrow 0} \frac{1}{\sqrt{L}} \sum_{k} e^{-\alpha|k|} e^{i k x} c_{k r}
$$

and

$$
\psi_{e l}(x)=\lim _{\alpha \searrow 0} \frac{1}{\sqrt{L}} \sum_{k} e^{-\alpha|k|} e^{i k x} c_{k l}
$$

The colons in Equation 3.1 denote that the field operators are ordered normally with respect to the ground state. It should be pointed out that the energy of the electrons is measured with respect to the Fermi energy $E_{f}=v_{f} k_{f}$. In the following the electrons whose energy dispersion has a positive gradient are referred to as right movers or right moving electrons while the electrons of the other sort are called left movers or left moving electrons. 


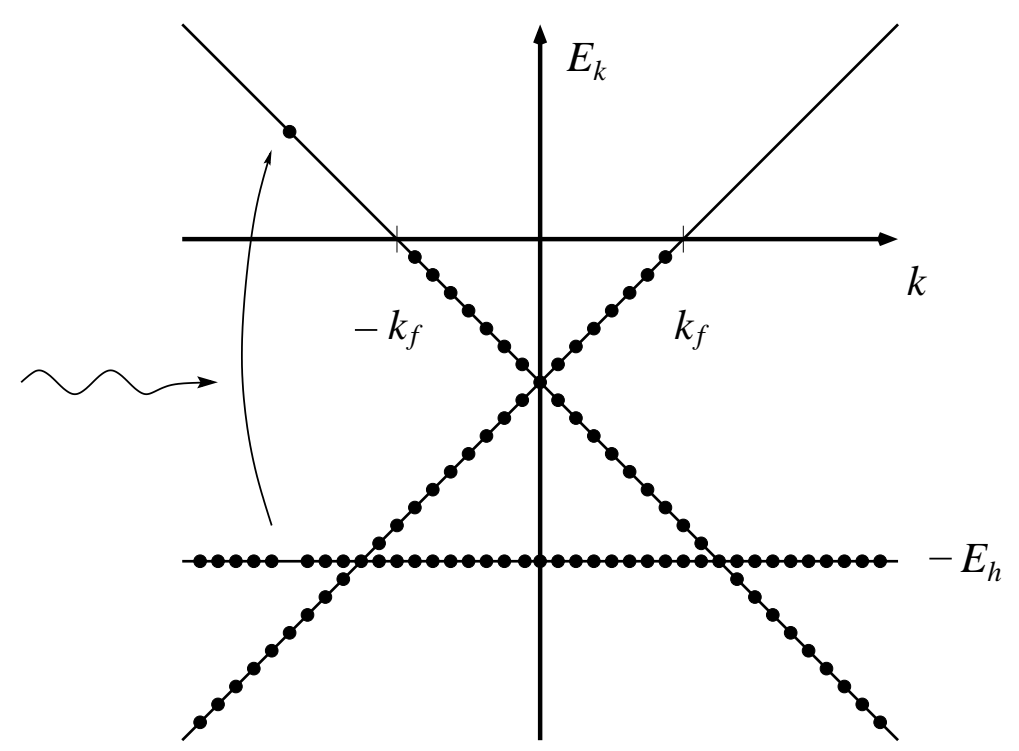

Figure 3.1: Graphical representation of the two-band model

In order to keep the model as simple as possible it is assumed that the valence holes have an infinite mass. Then their free Hamiltonian has the following form

$$
H_{0}^{h}=E_{h} \int_{-\frac{L}{2}}^{\frac{L}{2}} d y \psi_{h}^{\dagger}(y) \psi_{h}(y)=E_{h} \sum_{k} d_{k}^{\dagger} d_{k} .
$$

The description of the many particle system becomes both more complicated and more interesting, if the repulsive interaction between the conduction electrons and the attractive interaction between the conduction electrons and the valence holes is taken into account. Since only the latter is responsible for the appearance of the Fermi-edge singularity, the intraband interaction will be neglected in the following calculations. The Hamiltonian for the electron-hole interaction satisfies the equation

$$
\begin{aligned}
V & =\int_{-\frac{L}{2}}^{\frac{L}{2}} d x \int_{-\frac{L}{2}}^{\frac{L}{2}} d y v(|x-y|): \psi_{e r}^{\dagger}(x) \psi_{e r}(x): \psi_{h}^{\dagger}(y) \psi_{h}(y) \\
& +\int_{-\frac{L}{2}}^{\frac{L}{2}} d x \int_{-\frac{L}{2}}^{\frac{L}{2}} d y v(|x-y|): \psi_{e l}^{\dagger}(x) \psi_{e l}(x): \psi_{h}^{\dagger}(y) \psi_{h}(y)
\end{aligned}
$$

where backward scattering processes, which would lead to transitions of conduction electrons from the left branch to the right branch and vice versa, have been omitted. This approximation can be made plausible by the argument that these processes are accompanied by large momentum transfers of the order of $2 k_{f}$ or $-2 k_{f}$ whereas the linearization of the electronic energy dispersion was based on the assumption that electron-hole scattering processes with a much smaller momentum transfer are dominant. As the holes do not change their position because of their infinite mass, the 
operator $V$ can be interpreted as a one-particle potential for the conduction electrons whose form is determined by the hole density.

The interaction between the external electromagnetic field and the many-body system is treated within the framework of the dipole approximation using the Hamiltonian

$$
H_{F}(t)=E(t)\left\{P^{\dagger}+P\right\}=E(t)\left\{P_{r}^{\dagger}+P_{r}\right\}+E(t)\left\{P_{l}^{\dagger}+P_{l}\right\} .
$$

where the polarization operators are defined by the relation

$$
P_{\sigma}=\int_{-\frac{L}{2}}^{\frac{L}{2}} d x \int_{-\frac{L}{2}}^{\frac{L}{2}} d y \mu_{\sigma}(x-y) \psi_{e \sigma}(x) \psi_{h}(y)
$$

with $\sigma=r, l$. The coupling functions $\mu_{\sigma}$ are directly related to the dipole matrix elements for the interband transitions. These coupling functions only depend on on the relative distance between the electron position $x$ and the hole position $y$ since it is assumed that the system is excited homogeneously. Consequently, the electron-hole pairs which are created or annihilated have a vanishing center-of-mass momentum and the polarization operators are diagonal in the $k$-representation:

$$
P_{\sigma}=\sum_{k} \int_{-\frac{L}{2}}^{\frac{L}{2}} d x \mu_{\sigma}(x) e^{i k x} c_{k \sigma} d_{-k}=\sum_{k} \tilde{\mu}_{\sigma}(k) c_{k \sigma} d_{-k}
$$

In the literature, the $k$-dependence of the dipole matrix elements is often neglected as the excitation is usually restricted to a small region of the spectrum. This approximation, however, leads to the divergence of the total polarization signal. In order to avoid this, the coupling functions are assumed to decrease exponentially for large positive and negative values of $k$ :

$$
\tilde{\mu}_{r}(k)=e^{-\Lambda\left|k-k_{f}\right|}=\tilde{\mu}_{l}(-k) .
$$

If the thermodynamic limit is performed, the functions $\mu_{r}$ and $\mu_{l}$ assume the shape of Lorentzian curves whose width is given by $\Lambda$ :

$$
\mu_{r}(x)=e^{-i k_{f} x} \frac{1}{\pi} \frac{\Lambda}{x^{2}+\Lambda^{2}}=e^{-i k_{f} x} d(x)=\mu_{l}^{*}(x) .
$$

\subsection{Bosonization}

Due to the linear energy dispersion of the conduction electrons it is possible to calculate the exact eigenstates of the many-body system in the presence of the electron-hole interaction. To this end, the free electronic Hamiltonian

$$
H_{0}^{e}=\sum_{k} v_{f}\left(k-k_{f}\right)\left\{c_{k r}^{\dagger} c_{k r}-\left\langle n_{k r}^{0}\right\rangle\right\}-\sum_{k} v_{f}\left(k+k_{f}\right)\left\{c_{k l}^{\dagger} c_{k l}-\left\langle n_{k l}^{0}\right\rangle\right\}
$$


is written with the help of the operators $(q>0)$

$$
b_{q r}=\sqrt{\frac{2 \pi}{L q}} \sum_{k} c_{k-q r}^{\dagger} c_{k r} \quad \text { and } \quad b_{q l}=\sqrt{\frac{2 \pi}{L q}} \sum_{k} c_{k+q l}^{\dagger} c_{k l},
$$

which obey bosonic commutation rules, and the number operators for the right movers and the left movers

$$
N_{r}=\sum_{k}\left\{c_{k r}^{\dagger} c_{k r}-\left\langle n_{k r}^{0}\right\rangle\right\} \quad \text { and } \quad N_{l}=\sum_{k}\left\{c_{k l}^{\dagger} c_{k l}-\left\langle n_{k l}^{0}\right\rangle\right\} .
$$

In the new representation the operator $H_{0}^{e}$ is given by the relation

$$
H_{0}^{e}=\sum_{q>0} v_{f} q\left\{b_{q r}^{\dagger} b_{q r}+b_{q l}^{\dagger} b_{q l}\right\}+v_{f} \frac{\pi}{L}\left\{N_{r}^{2}+N_{l}^{2}\right\} .
$$

As the bosonic operators commute with the number operators the two different terms of which the free electronic Hamiltonian is composed can be discussed separately. The first term can be interpreted as an infinite sum of harmonic oscillators and describes the excitation spectrum for a system with a fixed number of particles. The second term describes the change of the energy of the ground state, if particles are removed or added. The advantage of the introduction of the bosonic operators becomes clear, if the interaction Hamiltonian is written as follows

$$
\begin{aligned}
V & =\sum_{q \neq 0} \frac{\tilde{v}(q)}{L} \sum_{k} c_{k+q r}^{\dagger} c_{k r} \int_{-\frac{L}{2}}^{\frac{L}{2}} d y e^{-i q y} \psi_{h}^{\dagger}(y) \psi_{h}(y) \\
& +\sum_{q \neq 0} \frac{\tilde{v}(q)}{L} \sum_{k} c_{k+q l}^{\dagger} c_{k l} \int_{-\frac{L}{2}}^{\frac{L}{2}} d y e^{-i q y} \psi_{h}^{\dagger}(y) \psi_{h}(y)
\end{aligned}
$$

where the Fourier coefficients of the potential are defined by the equation

$$
\tilde{v}(q)=\int_{-\frac{L}{2}}^{\frac{L}{2}} d x v(|x|) e^{-i q x}
$$

and it is assumed that the homogeneous part $\tilde{v}(0)$ is compensated by the charge background. Obviously, the expression on the right-hand side of Equation 3.15 can be simplified considerably, if the electronic densities are written with the help of the bosonic operators. The new representation of the operator $V$ then reads

$$
\begin{aligned}
V & =\int_{-\frac{L}{2}}^{\frac{L}{2}} d y \sum_{q>0} v_{f} q\left\{\Phi_{q}^{*}(y) b_{q r}^{\dagger}+\Phi_{q}(y) b_{q r}\right\} \psi_{h}^{\dagger}(y) \psi_{h}(y) \\
& +\int_{-\frac{L}{2}}^{\frac{L}{2}} d y \sum_{q>0} v_{f} q\left\{\Phi_{q}(y) b_{q l}^{\dagger}+\Phi_{q}^{*}(y) b_{q l}\right\} \psi_{h}^{\dagger}(y) \psi_{h}(y) \\
& =\int_{-\frac{L}{2}}^{\frac{L}{2}} d y V_{e}(y) \psi_{h}^{\dagger}(y) \psi_{h}(y)
\end{aligned}
$$


where the function $\Phi_{q}(y)$ is defined by

$$
\Phi_{q}(y)=\frac{\tilde{v}(q)}{2 \pi v_{f}} \sqrt{\frac{2 \pi}{L q}} e^{i q y} .
$$

It has already been mentioned that the dynamics of the valence holes is not influenced by the conduction electrons because of the infinite mass of the former. Therefore it is justified to treat the position $y$ of a valence hole as a constant parameter, which only determines the center of the potential $v(|x-y|)$ which is created by this hole. The Hamiltonian for the conduction electrons then includes the potential operator $V_{e}(y)$ which consists of linear combinations of the bosonic operators $b_{q \sigma}$ and $b_{q \sigma}^{\dagger}$.

In order to diagonalize the total electron Hamiltonian in the presence of an external potential, it is necessary to introduce the following canonical transformation

$$
\mathcal{S}(y)=e^{i\left\{\chi_{l}^{\dagger}(y)+\chi_{l}(y)\right\}} e^{i\left\{\chi_{r}^{\dagger}(y)+\chi_{r}(y)\right\}}
$$

with the auxiliary operators

$$
\chi_{r}(y)=-i \sum_{q>0} \frac{\tilde{v}(q)}{2 \pi v_{f}} e^{i q y} \sqrt{\frac{2 \pi}{L q}} b_{q r}=-i \sum_{q>0} \Phi_{q}(y) b_{q r}
$$

and

$$
\chi_{l}(y)=-i \sum_{q>0} \frac{\tilde{v}(q)}{2 \pi v_{f}} e^{-i q y} \sqrt{\frac{2 \pi}{L q}} b_{q l}=-i \sum_{q>0} \Phi_{q}^{*}(y) b_{q l} .
$$

The transformation behavior of the bosonic annihilation operators is given by

$$
\begin{aligned}
& \mathcal{S}^{\dagger}(y) b_{q r} \mathcal{S}(y)=b_{q r}-\Phi_{q}^{*}(y), \\
& \mathcal{S}^{\dagger}(y) b_{q l} \mathcal{S}(y)=b_{q l}-\Phi_{q}(y)
\end{aligned}
$$

If the conduction electrons are exposed to the potential of a hole at the position $y$, the electronic Hamiltonian $H_{0}^{e}+V_{e}(y)$ can be reduced to the free electron Hamiltonian $H_{0}^{e}$ by applying the canonical transformation from Equation 3.19:

$$
\mathcal{S}^{\dagger}(y)\left\{H_{0}^{e}+V_{e}(y)\right\} \mathcal{S}(y)=H_{0}^{e}-\varepsilon(0)
$$

The energy spectrum of $H_{0}^{e}+V_{e}(y)$ has the same structure as the free spectrum. Only the energy of the ground state is shifted by the value $-\varepsilon(0)$ where the energy function $\varepsilon(y)$ is determined by the equation

$$
\varepsilon(y)=2 v_{f} \sum_{q>0} \frac{2 \pi}{L} \frac{\tilde{v}^{2}(q)}{\left(2 \pi v_{f}\right)^{2}} \cos (q y) .
$$


In the presence of two holes at the positions $y$ and $y^{\prime}$, two canonical transformations are necessary to diagonalize the total electronic Hamiltonian. The result is then given by

$$
\mathcal{S}^{\dagger}\left(y^{\prime}\right) \mathcal{S}^{\dagger}(y)\left\{H_{0}^{e}+V_{e}(y)+V_{e}\left(y^{\prime}\right)\right\} \mathcal{S}(y) \mathcal{S}\left(y^{\prime}\right)=H_{0}^{e}-2 \varepsilon(0)-2 \varepsilon\left(y^{\prime}-y\right)
$$

where the energy shift of the ground state depends on the relative distance between the two holes. The result can easily be generalized to the case of three or more holes. For the following calculations, however, only the relations from Equations 3.24 and 3.26 are needed.

If the optical response of the system to an external laser field is calculated, it is necessary to evaluate spatial correlation functions containing products of two and four electronic field operators. To this end, these field operators are expressed with the help of the bosonic operators as follows

$$
\begin{aligned}
& \psi_{e r}(x)=\frac{1}{\sqrt{L}} e^{i x k_{f}} e^{i \frac{\pi}{L} x N_{r}} U_{r} e^{i \frac{\pi}{L} x N_{r}} e^{i \varphi_{r}^{\dagger}(x)} e^{i \varphi_{r}(x)}, \\
& \psi_{e l}(x)=\frac{1}{\sqrt{L}} e^{-i x k_{f}} e^{-i \frac{\pi}{L} x N_{l}} U_{l} e^{-i \frac{\pi}{L} x N_{l}} e^{i \varphi_{l}^{\dagger}(x)} e^{i \varphi_{l}(x)}
\end{aligned}
$$

where the auxiliary fields $\varphi_{r}(x)$ and $\varphi_{l}(x)$ are defined by the equations

$$
\varphi_{r}(x)=\lim _{\alpha \searrow 0}-i \sum_{q>0} e^{-\alpha q} e^{i q x} \sqrt{\frac{2 \pi}{L q}} b_{q r}
$$

and

$$
\varphi_{l}(x)=\lim _{\alpha \searrow 0}-i \sum_{q>0} e^{-\alpha q} e^{-i q x} \sqrt{\frac{2 \pi}{L q}} b_{q l} .
$$

The unitary operators $U_{r}$ and $U_{l}$ which appear on the right-hand sides of Equations 3.27 and 3.28 obey the following commutator rules

$$
\begin{aligned}
& {\left[U_{\sigma}, N_{\sigma^{\prime}}\right]=\delta_{\sigma ; \sigma^{\prime}} U_{\sigma},} \\
& {\left[U_{\sigma}, b_{q \sigma^{\prime}}\right]=\left[U_{\sigma}, b_{q \sigma^{\prime}}^{\dagger}\right]=0}
\end{aligned}
$$

with $\sigma, \sigma^{\prime} \in\{r, l\}$. In addition to that they satisfy the anti-commutator relations

$$
\left\{U_{r}, U_{l}\right\}=0 \quad \text { and } \quad\left\{U_{r}, U_{l}^{\dagger}\right\}=0 .
$$

Their introduction completes the operator algebra of the bosonic representation. A more detailed discussion of the different aspects of the bosonization can be found in F. D. Haldane's comprehensive article [17]. 


\subsection{Time-Dependent Perturbation Theory}

In the following the excitation of the many-body system by an external field will be described within the framework of the time-dependent perturbation theory. It is assumed that the system is in the ground state $\left|\Omega_{0}\right\rangle$ of the Hamiltonian $\tilde{H}=H_{0}^{e}+H_{0}^{h}+V$ at a given initial time $t_{0}$. That means that the valence band is completely filled while the electrons in the conduction band occupy all energy eigenstates up to the Fermi energy $E_{f}$. The dynamics of the system for $t \geq t_{0}$ is described by the Hamiltonian $H(t)=\tilde{H}+H_{F}(t)$. The corresponding time-development operator $U\left(t, t_{0}\right)$ satisfies the equations

$$
\begin{aligned}
i \frac{d}{d t} U\left(t, t_{0}\right) & =\left\{\tilde{H}+H_{F}(t)\right\} U\left(t, t_{0}\right), \\
U\left(t_{0}, t_{0}\right) & =\mathbf{1} .
\end{aligned}
$$

If the operator $U$ is expanded in a Dyson series with respect to the operator $H_{F}(t)$, the following equation

$$
U\left(t, t_{0}\right)=e^{-i\left(t-t_{0}\right) \tilde{H}} T\left[e^{-i \int_{t_{0}}^{t} d \tau \tilde{H}_{F}(\tau)}\right]
$$

can be derived (confer Equation 2.49). The operator $\tilde{H}_{F}(t)$ represents $H_{F}(t)$ in the interaction picture with respect to the Hamiltonian $\tilde{H}$. Equation 3.36 can be used as a starting point for the calculation of the system's linear and nonlinear response to the excitation by the external field. If $O$ is an arbitrary operator of the many-body-system, the following equation

$$
\begin{aligned}
\left\langle\Omega_{0}|O(t)| \Omega_{0}\right\rangle & =\left\langle\Omega_{0}\left|U^{\dagger}\left(t, t_{0}\right) O U\left(t, t_{0}\right)\right| \Omega_{0}\right\rangle \\
& =\left\langle\Omega_{0}\left|T_{\mathcal{C}}\left[\tilde{O}(t) e^{-i \int_{\mathcal{C}} d \tau \tilde{H}_{F}(\tau)}\right]\right| \Omega_{0}\right\rangle
\end{aligned}
$$

holds. The definitions for the contour-ordering-operator $T_{\mathcal{C}}$ and the Keldysh contour $\mathcal{C}$ have already been given in Section 2.4 .

The optical response of the material is determined by the dynamics of the polarization operator $P$ which can be calculated with the help of Equation 3.37. Since the number operators for valence holes and conduction electrons commute with the Hamiltonian $\tilde{H}$ and since there are no holes in the ground state $\left|\Omega_{0}\right\rangle$ the perturbation series for $P$ contains only terms which are proportional to odd powers of the external field $E$. The linear part of the polarization is given by

$$
P^{(1)}(t)=(-i) \int_{t_{0}}^{t} d \tau E(\tau)\left\langle\Omega_{0}\left|\tilde{P}(t) \tilde{P}^{\dagger}(\tau)\right| \Omega_{0}\right\rangle
$$

while the part of the polarization which is proportional to the cube of the external field 
satisfies the following equation

$$
\begin{aligned}
& P^{(3)}(t) \\
& =(-i)^{3} \int_{t_{0}}^{t} d \tau_{1} E\left(\tau_{1}\right) \int_{t_{0}}^{\tau_{1}} d \tau_{2} E\left(\tau_{2}\right) \int_{t_{0}}^{\tau_{2}} d \tau_{3} E\left(\tau_{3}\right)\left\langle\Omega_{0}\left|\tilde{P}(t) \tilde{P}^{\dagger}\left(\tau_{1}\right) \tilde{P}\left(\tau_{2}\right) \tilde{P}^{\dagger}\left(\tau_{3}\right)\right| \Omega_{0}\right\rangle \\
& +(-i)^{3} \int_{t_{0}}^{t} d \tau_{1} E\left(\tau_{1}\right) \int_{t_{0}}^{\tau_{1}} d \tau_{2} E\left(\tau_{2}\right) \int_{t_{0}}^{t} d \tau_{3} E\left(\tau_{3}\right)\left\langle\Omega_{0}\left|\tilde{P}\left(\tau_{2}\right) \tilde{P}^{\dagger}\left(\tau_{1}\right) \tilde{P}(t) \tilde{P}^{\dagger}\left(\tau_{3}\right)\right| \Omega_{0}\right\rangle \\
& +(-i)^{3} \int_{t_{0}}^{t} d \tau_{1} E\left(\tau_{1}\right) \int_{t_{0}}^{\tau_{1}} d \tau_{2} E\left(\tau_{2}\right) \int_{t_{0}}^{\tau_{2}} d \tau_{3} E\left(\tau_{3}\right)\left\langle\Omega_{0}\left|\tilde{P}(t) \tilde{P}\left(\tau_{1}\right) \tilde{P}^{\dagger}\left(\tau_{2}\right) \tilde{P}^{\dagger}\left(\tau_{3}\right)\right| \Omega_{0}\right\rangle \\
& -(-i)^{3} \int_{t_{0}}^{t} d \tau_{1} E\left(\tau_{1}\right) \int_{t_{0}}^{t} d \tau_{2} E\left(\tau_{2}\right) \int_{t_{0}}^{\tau_{2}} d \tau_{3} E\left(\tau_{3}\right)\left\langle\Omega_{0}\left|\tilde{P}\left(\tau_{1}\right) \tilde{P}(t) \tilde{P}^{\dagger}\left(\tau_{2}\right) \tilde{P}^{\dagger}\left(\tau_{3}\right)\right| \Omega_{0}\right\rangle .
\end{aligned}
$$

The two kinds of four-point response function which appear on the right-hand side of Equation 3.39 can be split up into connected and disconnected parts where the connected parts are defined by the equations

$$
\begin{aligned}
\left\langle\Omega_{0}\left|\tilde{P}\left(t_{1}\right) \tilde{P}^{\dagger}\left(t_{2}\right) \tilde{P}\left(t_{3}\right) \tilde{P}^{\dagger}\left(t_{4}\right)\right| \Omega_{0}\right\rangle_{\mathrm{c}} & =\left\langle\Omega_{0}\left|\tilde{P}\left(t_{1}\right) \tilde{P}^{\dagger}\left(t_{2}\right) \tilde{P}\left(t_{3}\right) \tilde{P}^{\dagger}\left(t_{4}\right)\right| \Omega_{0}\right\rangle \\
& -\left\langle\Omega_{0}\left|\tilde{P}\left(t_{1}\right) \tilde{P}^{\dagger}\left(t_{2}\right)\right| \Omega_{0}\right\rangle\left\langle\Omega_{0}\left|\tilde{P}\left(t_{3}\right) \tilde{P}^{\dagger}\left(t_{4}\right)\right| \Omega_{0}\right\rangle
\end{aligned}
$$

and

$$
\begin{aligned}
\left\langle\Omega_{0}\left|\tilde{P}\left(t_{1}\right) \tilde{P}\left(t_{2}\right) \tilde{P}^{\dagger}\left(t_{3}\right) \tilde{P}^{\dagger}\left(t_{4}\right)\right| \Omega_{0}\right\rangle_{\mathrm{c}} & =\left\langle\Omega_{0}\left|\tilde{P}\left(t_{1}\right) \tilde{P}\left(t_{2}\right) \tilde{P}^{\dagger}\left(t_{3}\right) \tilde{P}^{\dagger}\left(t_{4}\right)\right| \Omega_{0}\right\rangle \\
& -\left\langle\Omega_{0}\left|\tilde{P}\left(t_{1}\right) \tilde{P}^{\dagger}\left(t_{4}\right)\right| \Omega_{0}\right\rangle\left\langle\Omega_{0}\left|\tilde{P}\left(t_{2}\right) \tilde{P}^{\dagger}\left(t_{3}\right)\right| \Omega_{0}\right\rangle \\
& -\left\langle\Omega_{0}\left|\tilde{P}\left(t_{1}\right) \tilde{P}^{\dagger}\left(t_{3}\right)\right| \Omega_{0}\right\rangle\left\langle\Omega_{0}\left|\tilde{P}\left(t_{2}\right) \tilde{P}^{\dagger}\left(t_{4}\right)\right| \Omega_{0}\right\rangle .
\end{aligned}
$$

By inserting Equations 3.40 and 3.41 in Equation 3.39 it can be shown that only the connected parts of the four-point response functions contribute to the signal. In analogy to the two-point response functions they diverge with the length of the system $L$ in the thermodynamic limit. As a consequence thereof the polarization density $p^{(3)}(t)=L^{-1} P^{(3)}$ gives a finite value for $L \rightarrow \infty$.

The theoretical model for the solid which has been introduced is incomplete since a number of interaction processes, which are responsible for the finite lifetime of the electronic excitations, have been neglected (spontaneous photon emission, electronphonon-scattering, ...). Their influence is taken into account by inserting phenomenological decay terms for the one-hole excitations $\left(\Gamma_{x}\right)$, the two-hole excitations $\left(\Gamma_{x x}\right)$ and for the pure electronic excitations $\left(\Gamma_{0}\right)$ in Equations 3.38 and 3.39. The resulting new equations for $P^{(1)}$ and $P^{(3)}$ are

$$
P^{(1)}(t)=(-i) \int_{t_{0}}^{t} d \tau e^{-\Gamma_{x}(t-\tau)} E(\tau)\left\langle\Omega_{0}\left|\tilde{P}(t) \tilde{P}^{\dagger}(\tau)\right| \Omega_{0}\right\rangle
$$


and

$$
\begin{aligned}
& P^{(3)}(t) \\
& =(-i)^{3} \int_{t_{0}}^{t} d \tau_{1} e^{-\Gamma_{x}\left(t-\tau_{1}\right)} E\left(\tau_{1}\right) \int_{t_{0}}^{\tau_{1}} d \tau_{2} e^{-\Gamma_{0}\left(\tau_{1}-\tau_{2}\right)} E\left(\tau_{2}\right) \int_{t_{0}}^{\tau_{2}} d \tau_{3} e^{-\Gamma_{x}\left(\tau_{2}-\tau_{3}\right)} E\left(\tau_{3}\right) \\
& \quad \times\left\langle\Omega_{0}\left|\tilde{P}(t) \tilde{P}^{\dagger}\left(\tau_{1}\right) \tilde{P}\left(\tau_{2}\right) \tilde{P}^{\dagger}\left(\tau_{3}\right)\right| \Omega_{0}\right\rangle_{\mathrm{c}} \\
& +(-i)^{3} \int_{t_{0}}^{t} d \tau_{1} e^{-\Gamma_{0}\left(t-\tau_{1}\right)} E\left(\tau_{1}\right) \int_{t_{0}}^{\tau_{1}} d \tau_{2} e^{-\Gamma_{x}\left(\tau_{1}-\tau_{2}\right)} E\left(\tau_{2}\right) \int_{t_{0}}^{t} d \tau_{3} e^{-\Gamma_{x}\left(t-\tau_{3}\right)} E\left(\tau_{3}\right) \\
& \quad \times\left\langle\Omega_{0}\left|\tilde{P}\left(\tau_{2}\right) \tilde{P}^{\dagger}\left(\tau_{1}\right) \tilde{P}(t) \tilde{P}^{\dagger}\left(\tau_{3}\right)\right| \Omega_{0}\right\rangle_{\mathrm{c}} \\
& +(-i)^{3} \int_{t_{0}}^{t} d \tau_{1} e^{-\Gamma_{x}\left(t-\tau_{1}\right)} E\left(\tau_{1}\right) \int_{t_{0}}^{\tau_{1}} d \tau_{2} e^{-\Gamma_{x x}\left(\tau_{1}-\tau_{2}\right)} E\left(\tau_{2}\right) \int_{t_{0}}^{\tau_{2}} d \tau_{3} e^{-\Gamma_{x}\left(\tau_{2}-\tau_{3}\right)} E\left(\tau_{3}\right) \\
& \quad \times\left\langle\Omega_{0}\left|\tilde{P}(t) \tilde{P}\left(\tau_{1}\right) \tilde{P}^{\dagger}\left(\tau_{2}\right) \tilde{P}^{\dagger}\left(\tau_{3}\right)\right| \Omega_{0}\right\rangle_{\mathrm{c}} \\
& -(-i)^{3} \int_{t_{0}}^{t} d \tau_{1} e^{-\Gamma_{x}\left(t-\tau_{1}\right)} E\left(\tau_{1}\right) \int_{t_{0}}^{t} d \tau_{2} e^{-\Gamma_{x x}\left(t-\tau_{2}\right)} E\left(\tau_{2}\right) \int_{t_{0}}^{\tau_{2}} d \tau_{3} e^{-\Gamma_{x}\left(\tau_{2}-\tau_{3}\right)} E\left(\tau_{3}\right) \\
& \quad \times\left\langle\Omega_{0}\left|\tilde{P}\left(\tau_{1}\right) \tilde{P}(t) \tilde{P}^{\dagger}\left(\tau_{2}\right) \tilde{P}^{\dagger}\left(\tau_{3}\right)\right| \Omega_{0}\right\rangle_{\mathrm{c} .}
\end{aligned}
$$

\subsection{Linear Response}

The characteristic properties of the linear optical absorption spectrum of metals and $\mathrm{n}$-doped semiconductors have been the principal reason for the interest in the model which has been presented in Section 3.1. Within the framework of this model the absorption spectrum $A(\omega)$ is related to the two-point response function through the relation

$$
A(\omega)=\lim _{L \rightarrow \infty} \operatorname{Im}\left\{\frac{i}{L} \int_{0}^{\infty} d t e^{i \omega t} e^{-\Gamma_{x} t}\left\langle\Omega_{0}\left|\tilde{P}(t) \tilde{P}^{\dagger}(0)\right| \Omega_{0}\right\rangle\right\} .
$$

The total two-point response function is given by the sum of the corresponding response functions for the left and right moving electrons. Due to the inversion symmetry of the model these functions satisfy the equation

$$
\left\langle\Omega_{0}\left|\tilde{P}_{r}\left(t_{1}\right) \tilde{P}_{r}^{\dagger}\left(t_{2}\right)\right| \Omega_{0}\right\rangle=\left\langle\Omega_{0}\left|\tilde{P}_{l}\left(t_{1}\right) \tilde{P}_{l}^{\dagger}\left(t_{2}\right)\right| \Omega_{0}\right\rangle .
$$

Therefore it is sufficient to calculate the two-point response function for the right moving electrons. It is related to the spatial correlation function of the corresponding field operators by the following identity

$$
\begin{aligned}
\left\langle\Omega_{0}\left|\tilde{P}_{r}\left(t_{1}\right) \tilde{P}_{r}^{\dagger}\left(t_{2}\right)\right| \Omega_{0}\right\rangle= & \int_{-\frac{L}{2}}^{\frac{L}{2}} d x_{1} \int_{-\frac{L}{2}}^{\frac{L}{2}} d y_{1} \int_{-\frac{L}{2}}^{\frac{L}{2}} d x_{2} \int_{-\frac{L}{2}}^{\frac{L}{2}} d y_{2} \mu_{r}\left(x_{1}-y_{1}\right) \mu_{r}^{*}\left(x_{2}-y_{2}\right) \\
& \times\left\langle\tilde{\psi}_{e r}\left(x_{1}, t_{1}\right) \tilde{\psi}_{h}\left(y_{1}, t_{1}\right) \tilde{\psi}_{h}^{\dagger}\left(y_{2}, t_{2}\right) \tilde{\psi}_{e r}^{\dagger}\left(x_{2}, t_{2}\right)\right\rangle_{0}
\end{aligned}
$$


where the brackets $\langle\cdots\rangle_{0}$ denote the expectation value with respect to the non-interacting ground state $\left|\Omega_{0}\right\rangle$. As the Hamiltonian $\tilde{H}$ is diagonal with respect to the hole states in the position representation the expectation value for the field operators of the holes can be split off and the following equation

$$
\begin{gathered}
\left\langle\tilde{\psi}_{e r}\left(x_{1}, t_{1}\right) \tilde{\psi}_{h}\left(y_{1}, t_{1}\right) \tilde{\psi}_{h}^{\dagger}\left(y_{2}, t_{2}\right) \tilde{\psi}_{e r}^{\dagger}\left(x_{2}, t_{2}\right)\right\rangle_{0}=\left\langle\psi_{h ; 0}\left(y_{1}, t_{1}\right) \psi_{h ; 0}^{\dagger}\left(y_{2}, t_{2}\right)\right\rangle_{0} \\
\times\left\langle e^{i t_{1} H_{0}^{e}} \psi_{e r}\left(x_{1}\right) e^{-i\left(t_{1}-t_{2}\right)\left\{H_{0}^{e}+V_{e}\left(y_{1}\right)\right\}} \psi_{e r}^{\dagger}\left(x_{2}\right) e^{-i t_{2} H_{0}^{e}}\right\rangle_{0}
\end{gathered}
$$

holds. With the help of the unitary operator which has been introduced in Section 3.2 the above expression can be transformed in such a way that only the free bosonic and fermionic fields are needed to describe the dynamics:

$$
\begin{aligned}
\left\langle\tilde{\psi}_{e r}\left(x_{1}, t_{1}\right) \tilde{\psi}_{h}\left(y_{1}, t_{1}\right) \tilde{\psi}_{h}^{\dagger}\left(y_{2}, t_{2}\right) \tilde{\psi}_{e r}^{\dagger}\left(x_{2}, t_{2}\right)\right\rangle_{0} \\
=e^{i\left(t_{1}-t_{2}\right) \varepsilon(0)}\left\langle\psi_{h ; 0}\left(y_{1}, t_{1}\right) \psi_{h ; 0}^{\dagger}\left(y_{2}, t_{2}\right)\right\rangle_{0} \\
\quad \times\left\langle e^{i t_{1} H_{0}^{e}} \psi_{e r}\left(x_{1}\right) \mathcal{S}\left(y_{1}\right) e^{-i\left(t_{1}-t_{2}\right) H_{0}^{e}} \mathcal{S}^{\dagger}\left(y_{1}\right) \psi_{e r}^{\dagger}\left(x_{2}\right) e^{-i t_{2} H_{0}^{e}}\right\rangle_{0} \\
=e^{i\left(t_{1}-t_{2}\right) \varepsilon(0)}\left\langle\psi_{h ; 0}\left(y_{1}, t_{1}\right) \psi_{h ; 0}^{\dagger}\left(y_{2}, t_{2}\right)\right\rangle_{0} \\
\quad \times\left\langle\psi_{e r ; 0}\left(x_{1}, t_{1}\right) \mathcal{S}_{0}\left(y_{1}, t_{1}\right) \mathcal{S}_{0}^{\dagger}\left(y_{1}, t_{2}\right) \psi_{e r ; 0}^{\dagger}\left(x_{2}, t_{2}\right)\right\rangle_{0} .
\end{aligned}
$$

By means of the Baker-Hausdorff formula and the commutator relations listed in Appendix $\mathrm{D}$ the following identity

$$
\begin{aligned}
& \left\langle\tilde{\psi}_{e r}\left(x_{1}, t_{1}\right) \tilde{\psi}_{h}\left(y_{1}, t_{1}\right) \tilde{\psi}_{h}^{\dagger}\left(y_{2}, t_{2}\right) \tilde{\psi}_{e r}^{\dagger}\left(x_{2}, t_{2}\right)\right\rangle_{0}=e^{2 H^{*}\left(v_{f}\left(t_{1}-t_{2}\right), 0\right)} \\
& \quad \times e^{G^{*}\left(v_{f}\left(t_{1}-t_{2}\right)-\left(x_{1}-y_{1}\right),-\left(x_{1}-y_{1}\right)\right)} e^{G^{*}\left(v_{f}\left(t_{1}-t_{2}\right)+\left(x_{2}-y_{1}\right),\left(x_{2}-y_{1}\right)\right)} \\
& \quad \times e^{i\left(t_{1}-t_{2}\right) \varepsilon(0)}\left\langle\psi_{h ; 0}\left(y_{1}, t_{1}\right) \psi_{h ; 0}^{\dagger}\left(y_{2}, t_{2}\right)\right\rangle_{0}\left\langle\psi_{e r ; 0}\left(x_{1}, t_{1}\right) \psi_{e r ; 0}^{\dagger}\left(x_{2}, t_{2}\right)\right\rangle_{0}
\end{aligned}
$$

is derived. Consequently, the response function can be written as follows

$$
\begin{aligned}
\left\langle\Omega_{0}\left|\tilde{P}_{r}\left(t_{1}\right) \tilde{P}_{r}^{\dagger}\left(t_{2}\right)\right| \Omega_{0}\right\rangle= & e^{-i\left(t_{1}-t_{2}\right)\left(E_{h}-\varepsilon(0)\right)} e^{2 H^{*}\left(v_{f}\left(t_{1}-t_{2}\right), 0\right)} \\
\times & \int_{-\frac{L}{2}}^{\frac{L}{2}} d y \int_{-\frac{L}{2}}^{\frac{L}{2}} d x_{1} \int_{-\frac{L}{2}}^{\frac{L}{2}} d x_{2} \mu_{r}\left(x_{1}-y\right) \mu_{r}^{*}\left(x_{2}-y\right) \\
& \times e^{G^{*}\left(v_{f}\left(t_{1}-t_{2}\right)-\left(x_{1}-y\right),-\left(x_{1}-y\right)\right)} e^{G^{*}\left(v_{f}\left(t_{1}-t_{2}\right)+\left(x_{2}-y\right),\left(x_{2}-y\right)\right)} \\
& \times\left\langle\psi_{e r ; 0}\left(x_{1}, t_{1}\right) \psi_{e r ; 0}^{\dagger}\left(x_{2}, t_{2}\right)\right\rangle_{0} .
\end{aligned}
$$

Since all functions are invariant with respect to the spatial translations $\pm L$ one integration can be carried out by means of a suitable substitution:

$$
\begin{aligned}
\frac{1}{L}\left\langle\Omega_{0}\left|\tilde{P}_{r}\left(t_{1}\right) \tilde{P}_{r}^{\dagger}\left(t_{2}\right)\right| \Omega_{0}\right\rangle= & e^{-i\left(t_{1}-t_{2}\right)\left(E_{h}-\varepsilon(0)\right)} e^{2 H^{*}\left(v_{f}\left(t_{1}-t_{2}\right), 0\right)} \\
\times & \int_{-\frac{L}{2}}^{\frac{L}{2}} d \tilde{x}_{1} \mu_{r}\left(\tilde{x}_{1}\right) \int_{-\frac{L}{2}}^{\frac{L}{2}} d \tilde{x}_{2} \mu_{r}^{*}\left(\tilde{x}_{2}\right)\left\langle\psi_{e r ; 0}\left(\tilde{x}_{1}, t_{1}\right) \psi_{e r ; 0}^{\dagger}\left(\tilde{x}_{2}, t_{2}\right)\right\rangle_{0} \\
& \times e^{G^{*}\left(v_{f}\left(t_{1}-t_{2}\right)-\tilde{x}_{1},-\tilde{x}_{1}\right)} e^{G^{*}\left(v_{f}\left(t_{1}-t_{2}\right)+\tilde{x}_{2}, \tilde{x}_{2}\right)} .
\end{aligned}
$$


As a consequence thereof, the thermodynamic limit can be performed easily and one obtains

$$
\begin{aligned}
& \lim _{L \rightarrow \infty} \frac{1}{L}\left\langle\Omega_{0}\left|\tilde{P}_{r}\left(t_{1}\right) \tilde{P}_{r}^{\dagger}\left(t_{2}\right)\right| \Omega_{0}\right\rangle=e^{-i\left(t_{1}-t_{2}\right)\left(E_{h}-\varepsilon(0)\right)} e^{2 H^{*}\left(v_{f}\left(t_{1}-t_{2}\right), 0\right)} \int_{0}^{\infty} \frac{d k}{2 \pi} e^{-i k v_{f}\left(t_{1}-t_{2}\right)} \\
& \quad \times \int_{-\infty}^{\infty} d \tilde{x}_{1} d\left(\tilde{x}_{1}\right) e^{i k \tilde{x}_{1}} e^{G\left(\tilde{x}_{1}-v_{f}\left(t_{1}-t_{2}\right), \tilde{x}_{1}\right)} \int_{-\infty}^{\infty} d \tilde{x}_{2} d\left(\tilde{x}_{2}\right) e^{-i k \tilde{x}_{2}} e^{G^{*}\left(\tilde{x}_{2}+v_{f}\left(t_{1}-t_{2}\right), \tilde{x}_{2}\right)}
\end{aligned}
$$

By using the auxiliary functions

$$
\begin{aligned}
& s_{X ; r}\left(t_{1}, t_{2}, x\right)=H^{*}\left(v_{f}\left(t_{1}-t_{2}\right), 0\right)+G^{*}\left(v_{f}\left(t_{1}-t_{2}\right)-x,-x\right), \\
& s_{X ; l}\left(t_{1}, t_{2}, x\right)=H^{*}\left(v_{f}\left(t_{1}-t_{2}\right), 0\right)+G^{*}\left(v_{f}\left(t_{1}-t_{2}\right)+x, x\right)
\end{aligned}
$$

Equation 3.52 can be written in a more compact form

$$
\begin{aligned}
& \lim _{L \rightarrow \infty} \frac{1}{L}\left\langle\Omega_{0}\left|\tilde{P}_{r}\left(t_{1}\right) \tilde{P}_{r}^{\dagger}\left(t_{2}\right)\right| \Omega_{0}\right\rangle=\left\langle\left\langle\tilde{P}_{r}\left(t_{1}\right) \tilde{P}_{r}^{\dagger}\left(t_{2}\right)\right\rangle\right\rangle=e^{-i\left(t_{1}-t_{2}\right)\left(E_{h}-\varepsilon(0)\right)} \\
& \times \int_{0}^{\infty} \frac{d k}{2 \pi} e^{i k v_{f}\left(t_{2}-t_{1}\right)} \int_{-\infty}^{\infty} d \tilde{x}_{1} d\left(\tilde{x}_{1}\right) e^{i k \tilde{x}_{1}} e^{s_{X ; r}\left(t_{1}, t_{2}, \tilde{x}_{1}\right)} \int_{-\infty}^{\infty} d \tilde{x}_{2} d\left(\tilde{x}_{2}\right) e^{-i k \tilde{x}_{2}} e^{s_{X ; r}^{*}\left(t_{2}, t_{1}, \tilde{x}_{2}\right)} \\
& =e^{-i\left(t_{1}-t_{2}\right)\left(E_{h}-\varepsilon(0)\right)} \\
& \times \int_{0}^{\infty} \frac{d k}{2 \pi} e^{i k v_{f}\left(t_{2}-t_{1}\right)} \int_{-\infty}^{\infty} d \tilde{x}_{1} d\left(\tilde{x}_{1}\right) e^{-i k \tilde{x}_{1}} e^{s_{X ; l}\left(t_{1}, t_{2}, \tilde{x}_{1}\right)} \int_{-\infty}^{\infty} d \tilde{x}_{2} d\left(\tilde{x}_{2}\right) e^{i k \tilde{x}_{2}} e^{s_{X ; l}^{*}\left(t_{2}, t_{1}, \tilde{x}_{2}\right)} .
\end{aligned}
$$

Generally, the two-point response functions cannot be calculated exactly because it is usually impossible to give the explicit solutions for the multi-dimensional integrals which appear in Equation 3.55. Therefore all exact expressions have to be approximated in order to obtain usable results.

If the auxiliary functions which appear in Equation 3.55 are slowly varying in comparison with the functions $d\left(\tilde{x}_{1}\right)$ and $d\left(\tilde{x}_{2}\right)$, it is justified to evaluate them at the positions $\tilde{x}_{1}=0$ and $\tilde{x}_{2}=0$ respectively. The condition is met, if the interaction potential is almost constant on the length scale which is determined by $\Lambda$. If the Fourier transform of the potential satisfies the equation $\tilde{v}(q)=\tilde{v}_{0} e^{-\lambda|q|}$, this requirement is equivalent to the relation $\lambda \gg \Lambda$. It should be pointed out that the approximated versions of the response functions are still exact, if the interaction potential vanishes.

The approximated two-point response function for the right-moving electrons satisfies the relation

$$
\begin{aligned}
\left\langle\left\langle\tilde{P}_{r}\left(t_{1}\right) \tilde{P}_{r}^{\dagger}\left(t_{2}\right)\right\rangle\right\rangle \approx & e^{-i\left(t_{1}-t_{2}\right)\left(E_{h}-\varepsilon(0)\right)} e^{s_{X ; r}\left(t_{1}, t_{2}, 0\right)} e^{s_{X ; r}^{*}\left(t_{2}, t_{1}, 0\right)} \\
& \times(2 \pi i)^{-1}\left(v_{f}\left(t_{1}-t_{2}\right)-2 \Lambda i\right)^{-1}
\end{aligned}
$$

where the last two factors result from the integration with respect to the wave vector $k$. All terms which are due to the electron-hole interaction can be combined in a factor in 
front of the non-interacting response function. As long as the difference between the two external time variables is large compared with the width of the Lorentzian curve $\left(\left|t_{1}-t_{2}\right| \gg v_{f}^{-1} \Lambda\right)$ the form of the response function is hardly affected by the finite extension of the dipole coupling function.

It has already been mentioned that the linear absorption spectrum $A(\omega)$ is directly related to the two-point response function from Equation 3.56. If the particles do not interact with each other, the absorption function is given by

$$
A(\omega)=\Theta\left(\omega-\omega_{T}\right) \frac{1}{v_{f}} e^{-2 \frac{\Lambda}{v_{f}}\left(\omega-\omega_{T}\right)}
$$

in the limit $\Gamma_{x} \rightarrow 0$. The function exhibits an absorption edge at the threshold energy $\omega_{T}=E_{h}$ and decays exponentially for $\omega>\omega_{T}$. In the presence of the electron-hole interaction the exact time behavior of the two-point response function depends on the actual form of the interaction potential $v(|x|)$. However, it is possible to give a general approximation for the response function with the help of the results from Appendix D, if $t \gg x_{\tilde{v}} v_{f}^{-1}$. The approximated response function then satisfies the relation

$$
\left\langle\left\langle\tilde{P}_{r}(t) \tilde{P}_{r}^{\dagger}(0)\right\rangle\right\rangle \approx(2 \pi i)^{-1} z^{*} e^{-i t\left(E_{h}-\varepsilon(0)\right)}\left(v_{f} t\right)^{-(1+\alpha)} .
$$

For large times the interaction contribution to the response function exhibits a powerlaw behavior with an exponent $\alpha$, which satisfies the equation

$$
\alpha=2 \frac{\tilde{v}(0)}{2 \pi v_{f}}+2 \frac{\tilde{v}^{2}(0)}{\left(2 \pi v_{f}\right)^{2}}=2 V_{0}+2 V_{0}^{2} .
$$

The complex constant is given by $z=e^{2 c_{H}+2 c_{G}} e^{i \frac{\pi}{2} \alpha}$. As it is assumed that the electronhole interaction is attractive, the interaction amplitude $V_{0}=\frac{\tilde{v}(0)}{\left(2 \pi v_{f}\right)}$ is negative. Consequently, the exponent $\alpha$ satisfies the relation $\alpha \geq-0.5$ where the minimal value is assumed, if $V_{0}=-0.5$. If the relations $-1<V_{0}<0$ are valid, the total response function decays more slowly than $t^{-1}$ and the expression for $A(\omega)$ exhibits an asymmetric algebraic singularity at the shifted threshold energy $\omega_{T}=E_{h}-\varepsilon(0)$ in the limit $\Gamma_{x} \rightarrow 0$. If it is assumed that the approximated expression for the two-point response function in Equation 3.58 is exact, the absorption function satisfies the relation

$$
A(\omega)=\Theta\left(\omega-\omega_{T}\right) \frac{1}{v_{f}} \frac{e^{2 c_{H}+2 c_{G}}}{\Gamma(1+\alpha)}\left(\frac{\omega-\omega_{T}}{v_{f}}\right)^{\alpha}
$$

where $\Gamma(\ldots)$ denotes the gamma function [7]. It can be shown that the difference between the exact absorption function and the expression on the right-hand side of Equation 3.60 remains finite in the vicinity of $\omega_{T}$. Therefore the above expression offers a good description of the leading behavior of $A(\omega)$ near the threshold.

If the interaction amplitude $V_{0}$ is smaller than -1 , the integral in Equation 3.44 remains finite for all frequencies $\omega$, if the damping constant $\Gamma_{x}$ is set equal to zero. Then the absorption spectrum near the threshold depends on the dynamics of the two-point 


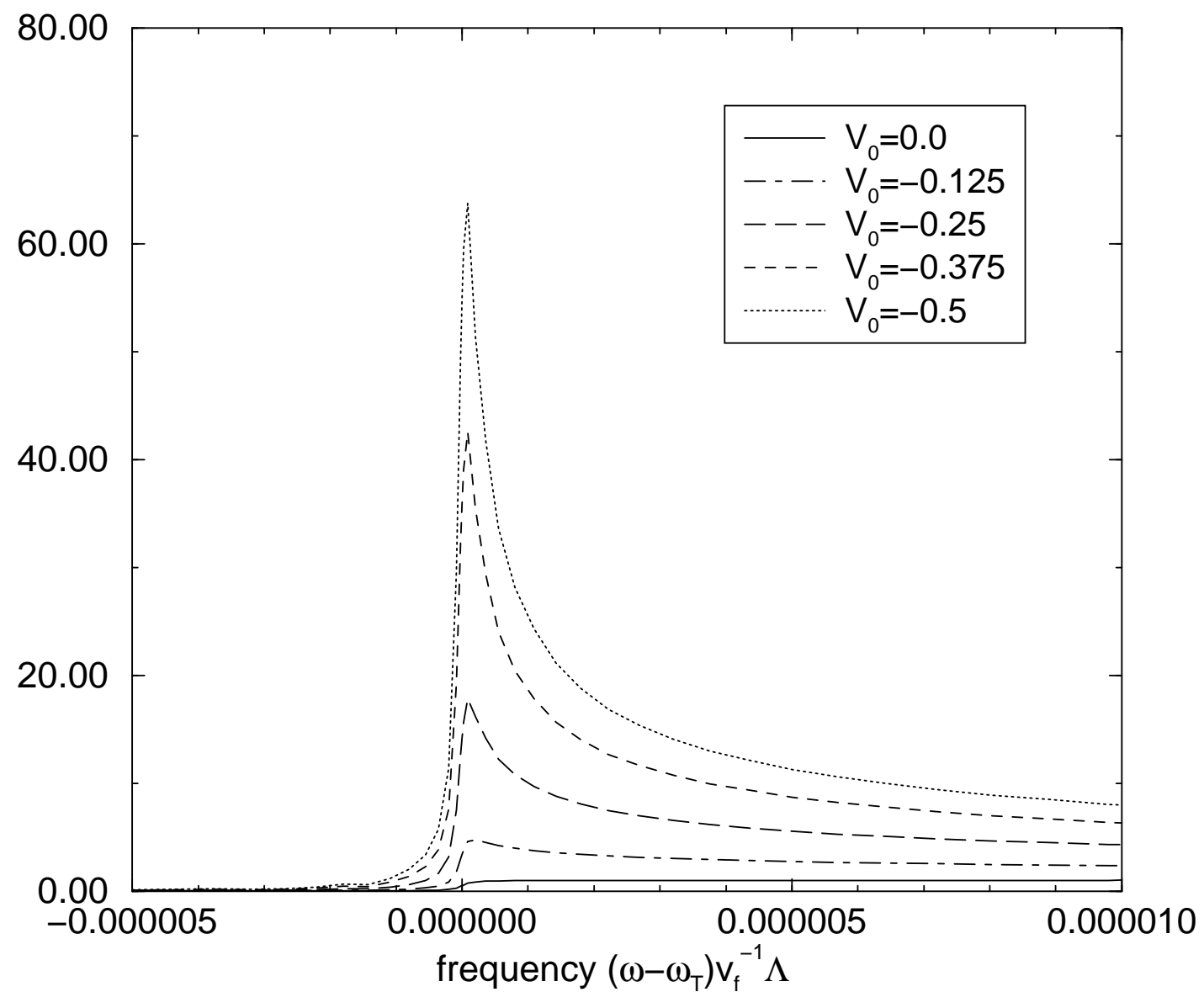

Figure 3.2: The linear absorption spectrum $A(\omega)$ for different interaction amplitudes $V_{0} \geq-0.5$, the excitonic damping constant is given by $\Gamma_{x}=2 \times 10^{-8} v_{f} \Lambda^{-1}$ and the width of the interaction potential is $\lambda=1000 \Lambda$

response function for all values of $t$ and it is impossible to derive a general approximation for the frequency behavior of $A(\omega)$ near the threshold which only depends on the interaction amplitude $V_{0}$.

The exponent $\alpha$ which determines the behavior of the absorption function near the renormalized Fermi-edge at $\omega=\omega_{T}$ is composed of two terms whose influences on the sign of $\alpha$ are diametrically opposed to each other.

The first term, which is proportional to the interaction amplitude $V_{0}$, is responsible for the singular behavior of $A(\omega)$ for small negative values of $V_{0}$. It is due to the interaction of the excited electron, which departs the valence band and becomes a conduction electron, and the hole which appears in the valence band as a consequence of this transition. A closer analysis of the expression for the two-point response function in Equation 3.56 shows that the term stems from the auxiliary function $G$.

The second term, which is related to the auxiliary function $H$, is proportional to the 
square of the interaction amplitude $V_{0}$ and leads to the suppression of the singularity for large negative values of $V_{0}$. It is related to the exponent of the so-called orthogonality catastrophe, the disappearance of the overlap between the free ground state of the many-body system and the ground state in the presence of a hole potential in the thermodynamic limit $[9,50]$. Within the framework of the Tomonaga-Luttinger model the overlap is given by

$$
\left\langle\Omega_{0}\left|\mathcal{S}^{\dagger}(0)\right| \Omega_{0}\right\rangle=e^{-\frac{1}{2}\left[\chi_{l}(0), \chi_{l}^{\dagger}(0)\right]} e^{-\frac{1}{2}\left[\chi_{r}(0), \chi_{r}^{\dagger}(0)\right]}=\exp \left\{-\sum_{q>0} \frac{2 \pi}{L q} \frac{\tilde{v}^{2}(q)}{\left(2 \pi v_{f}\right)^{2}}\right\}
$$

where it is assumed that the valence hole is located at $y=0$. The behavior of the overlap in the thermodynamic limit can be determined, if the expression on the righthand side of Equation 3.61 is written in the following way

$$
\left\langle\Omega_{0}\left|\mathcal{S}^{\dagger}(0)\right| \Omega_{0}\right\rangle=\exp \left\{-\sum_{q>0} \frac{2 \pi}{L q} \frac{\tilde{v}^{2}(q)-\tilde{v}^{2}(0) e^{-q}}{\left(2 \pi v_{f}\right)^{2}}\right\}\left\{1-e^{-\frac{2 \pi}{L}}\right\}^{\frac{\tilde{v}^{2}(0)}{\left(2 \pi v_{f}\right)^{2}}} .
$$

In the thermodynamic limit the value of the overlap satisfies the equation

$$
\left\langle\Omega_{0}\left|\mathcal{S}^{\dagger}(0)\right| \Omega_{0}\right\rangle=\exp \left\{-\int_{0}^{\infty} \frac{d q}{q} \frac{\tilde{v}^{2}(q)-\tilde{v}^{2}(0) e^{-q}}{\left(2 \pi v_{f}\right)^{2}}\right\}\left\{\frac{2 \pi}{L}\right\}^{\frac{\tilde{\tilde{n}}^{2}(0)}{\left(2 \pi v_{f}\right)^{2}}}
$$

and vanishes like $L^{-V_{0}^{2}}$.

In Figures 3.2 and 3.3 the absorption spectrum $A(\omega)$ is plotted for different values of the interaction amplitude and a non-vanishing excitonic damping constant using the expression on the left-hand side of Equation 3.56. When calculating the curves it is assumed that the interaction potential $v(|x|)$ satisfies the equation

$$
v(|x|)=\frac{\tilde{v}_{0}}{\pi} \frac{\lambda}{x^{2}+\lambda^{2}}
$$

in the thermodynamic limit where $\tilde{v}_{0}<0$ and $\lambda>0$. The Fourier transform of the potential is then given by $\tilde{v}(q)=\tilde{v}_{0} e^{-\lambda|q|}$. The special form of the interaction potential makes it possible to give the exact form of the auxiliary functions $G$ and $H$ (see Appendix D for details). The width of the interaction potential is chosen to be much larger than the width of the dipole coupling functions $(\lambda \gg \Lambda)$. This is consistent with the assumption which has been made when approximating the exact two-point response function from Equation 3.55. The values of $V_{0}$ which are used for the calculation of the curves in Figure 3.2 are chosen from the interval $[-0.5,0]$ whereas the functions in Figure 3.3 have been calculated with values of $V_{0}$ which are smaller or equal to -0.5 . If the interaction amplitude approaches $V_{0}=-0.5$ from above, the absorption edge singularity at $\omega=\omega_{T}$ becomes steeper. The trend is reversed for smaller values of $V_{0}$ and the singularity is suppressed entirely, if $V_{0}=-1$. 


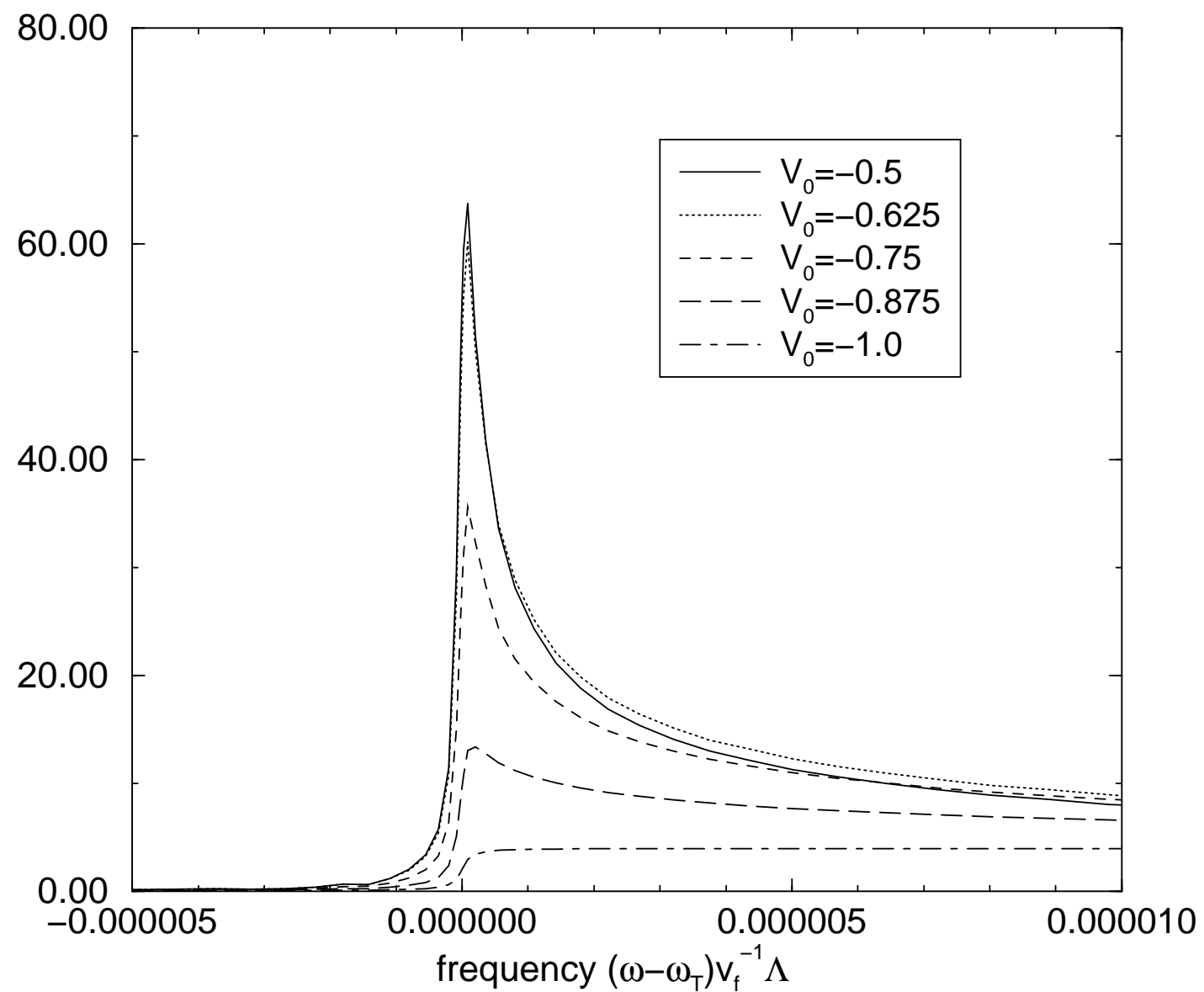

Figure 3.3: The linear absorption spectrum $A(\omega)$ for different interaction amplitudes $V_{0} \leq-0.5$, the excitonic damping constant is given by $\Gamma_{x}=2 \times 10^{-8} v_{f} \Lambda^{-1}$ and the width of the interaction potential is $\lambda=1000 \Lambda$

This behavior can be explained satisfactorily by means of the approximated expression for $A(\omega)$ in Equation 3.60 since the exponent $\alpha$ which determines the grade of the singularity assumes a minimal value at $V_{0}=-0.5$ and is a symmetric function of $V_{0}$ with respect to this point. A comparison of the different absorption functions shows that the curves in Figure 3.2 do not have the same form as their counterparts with the same exponent $\alpha$ in Figure 3.3. This is due to the fact that the function $e^{2 c_{H}+2 c_{G}}$ exhibits no inversion symmetry with respect to $V_{0}=-0.5$.

Finally, it should be pointed out that the general interpretation of the absorption function $A(\omega)$ with the help of Equation 3.60 is only possible, if the excitonic damping is weak enough to allow the observation of the algebraic decay of the two-point response function in Equation 3.44. In order to illustrate this, the function $A(\omega)$ is plotted for different values of $\Gamma_{x}$ in Figure 3.4 where the form of the interaction function is determined by Equation 3.64 and the interaction amplitude is given by $V_{0}=-0.5$. 


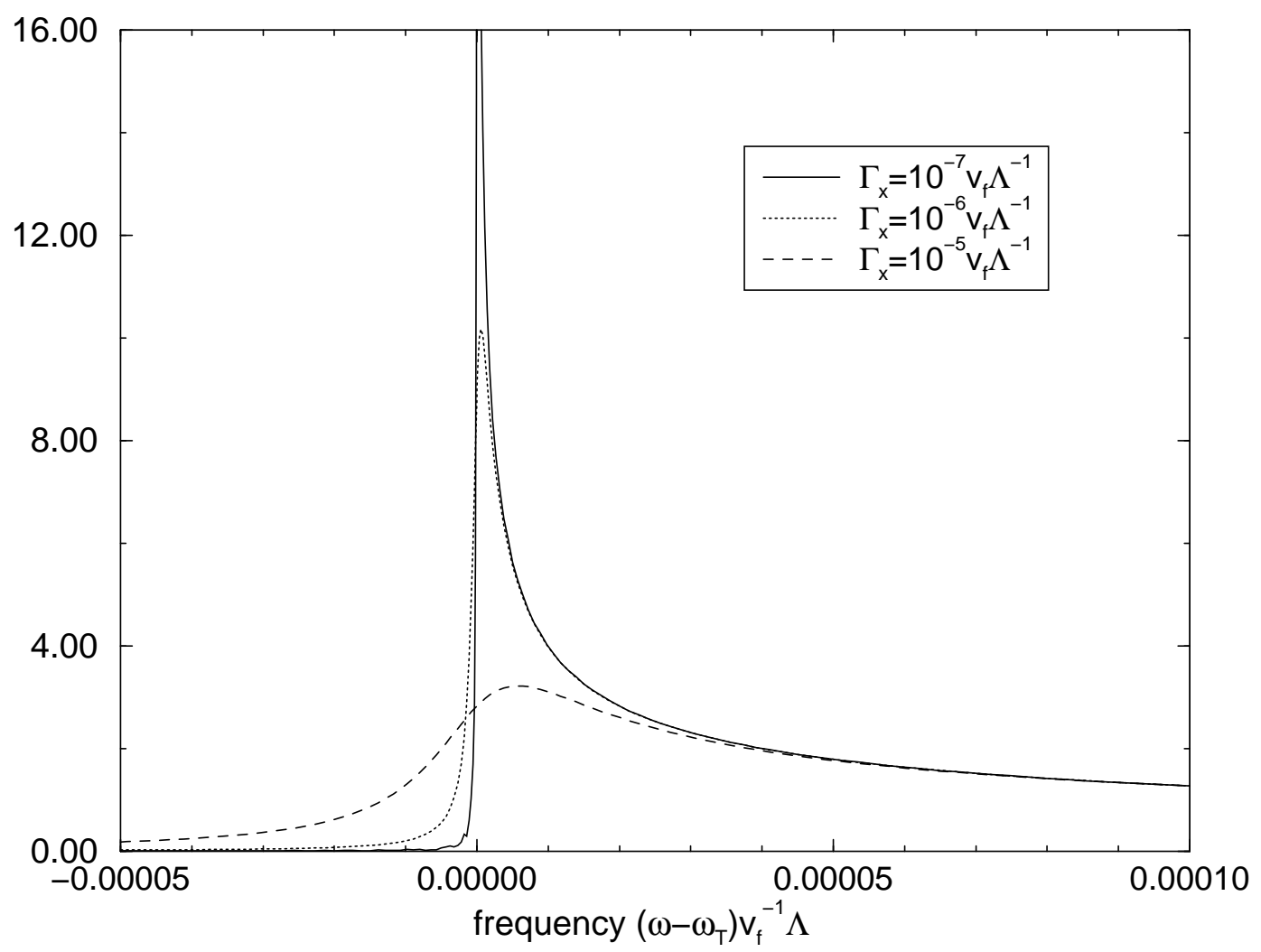

Figure 3.4: The linear absorption spectrum $A(\omega)$ for different excitonic damping constants $\Gamma_{x}$, the interaction amplitude is given by $V_{0}=-0.5$ and the width of the interaction potential is $\lambda=1000 \Lambda$

As expected, the absorption edge is suppressed, if the value of the damping constant is increased. If the model parameters are similar to those which have been used in this section, the Fermi-edge singularity can be observed, provided that the excitonic damping constant satisfies the relation $\Gamma_{x} \leq 10^{-7} v_{f} \Lambda^{-1}$.

\subsection{Nonlinear Response}

The general expression for the part of the polarization which is proportional to the cube of the external field (see Equation 3.43) can be used for the description of different kinds of nonlinear optical experiments. In this work it is employed in order to study coherent transient effects in a four-wave-mixing experiment whose experimental setup is shown in Figure 3.5. It is assumed that the external field $E(t)$ is composed of two pulses $E_{1}(t)$ and $E_{2}(t)$ with

$$
E_{1}(t)=\mathcal{E}(t) \cos \left(\omega t-\vec{k}_{1} \vec{R}\right)
$$


and

$$
E_{2}(t)=\mathcal{E}(t+T) \cos \left(\omega t-\vec{k}_{2} \vec{R}\right)
$$

These pulses propagate into different directions which are determined by the wave vectors $\vec{k}_{1}$ and $\vec{k}_{2}$. The vector $\vec{R}$ denotes the macroscopic position of the electronic system and determines the phase difference between two arbitrary points of the probe. It should not be confused with the microscopic positions of the electrons or holes. The central frequency $\omega$ is chosen to be equal to the threshold energy $\omega_{T}$ while the envelope function $\mathcal{E}(t)$ is assumed to have a sharp peak at $t=0$. If the rotating wave approximation is applied, only the resonant terms in $H_{F}(t)$ are taken into account and the following operator

$$
H_{F}(t) \approx \bar{E}^{*}(t) P^{\dagger}+\bar{E}(t) P
$$

with

$$
\bar{E}(t)=\frac{1}{2} e^{i \omega t}\left\{\mathcal{E}(t) e^{-i \vec{k}_{1} \vec{R}}+\mathcal{E}(t+T) e^{-i \vec{k}_{2} \vec{R}}\right\}
$$

is used for the description of the matter-field interaction.

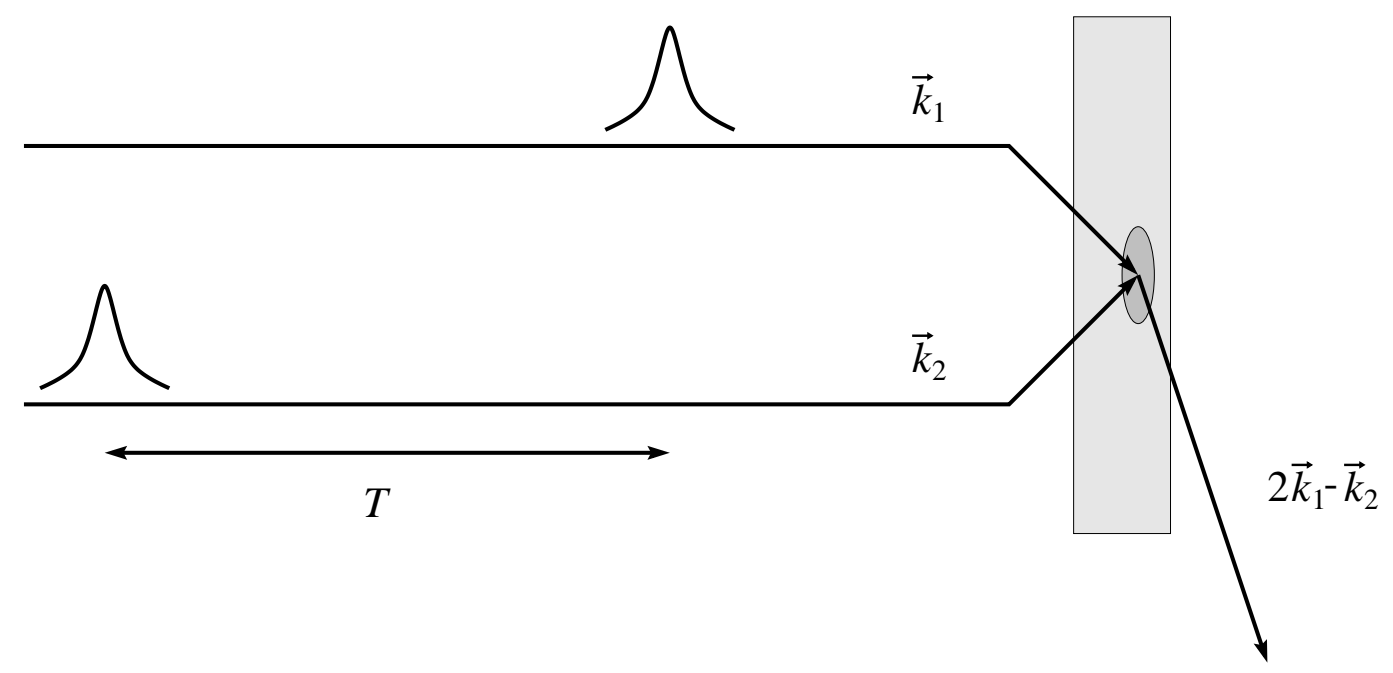

Figure 3.5: The standard setup for a four-wave-mixing experiment with two excitation pulses

As long as the delay time $T$ is of the same order of magnitude as the decay times of the system $\left(\Gamma_{i} T \approx 1\right.$ with $\left.i=0, x, x x\right)$ the coherent superposition of the two pulses in the nonlinear regime leads to the generation of several additional outgoing signals whose wave vectors and frequencies are determined by the corresponding phase matching conditions. In the following the investigation is concentrated on the 
signal which propagates in the $2 \vec{k}_{1}-\vec{k}_{2}$-direction. It is proportional to the following contribution to the nonlinear polarization

$$
\begin{aligned}
& P_{2 \vec{k}_{1}-\vec{k}_{2}}^{(3)}(t)=\frac{(-i)^{3}}{2^{3}} e^{-i\left(\omega t-\left(2 \vec{k}_{1}-\vec{k}_{2}\right) \vec{R}\right)} \\
& \times\left\{\int_{-\infty}^{t} d \tau_{1} e^{-\Gamma_{x}\left(t-\tau_{1}\right)} \mathcal{E}\left(\tau_{1}\right) \int_{-\infty}^{\tau_{1}} d \tau_{2} e^{-\Gamma_{0}\left(\tau_{1}-\tau_{2}\right)} \mathcal{E}\left(\tau_{2}+T\right) \int_{-\infty}^{\tau_{2}} d \tau_{3} e^{-\Gamma_{x}\left(\tau_{2}-\tau_{3}\right)} \mathcal{E}\left(\tau_{3}\right)\right. \\
& \times e^{i \omega\left(t-\tau_{1}+\tau_{2}-\tau_{3}\right)}\left\langle\Omega_{0}\left|\tilde{P}(t) \tilde{P}^{\dagger}\left(\tau_{1}\right) \tilde{P}\left(\tau_{2}\right) \tilde{P}^{\dagger}\left(\tau_{3}\right)\right| \Omega_{0}\right\rangle_{\mathrm{c}} \\
&+\int_{-\infty}^{t} d \tau_{1} e^{-\Gamma_{0}\left(t-\tau_{1}\right)} \mathcal{E}\left(\tau_{1}\right) \int_{-\infty}^{\tau_{1}} d \tau_{2} e^{-\Gamma_{x}\left(\tau_{1}-\tau_{2}\right)} \mathcal{E}\left(\tau_{2}+T\right) \int_{-\infty}^{t} d \tau_{3} e^{-\Gamma_{x}\left(t-\tau_{3}\right)} \mathcal{E}\left(\tau_{3}\right) \\
& \times e^{i \omega\left(t-\tau_{1}+\tau_{2}-\tau_{3}\right)}\left\langle\Omega_{0}\left|\tilde{P}\left(\tau_{2}\right) \tilde{P}^{\dagger}\left(\tau_{1}\right) \tilde{P}(t) \tilde{P}^{\dagger}\left(\tau_{3}\right)\right| \Omega_{0}\right\rangle_{\mathrm{c}} \\
&+ \int_{-\infty}^{t} d \tau_{1} e^{-\Gamma_{x}\left(t-\tau_{1}\right)} \mathcal{E}\left(\tau_{1}+T\right) \int_{-\infty}^{\tau_{1}} d \tau_{2} e^{-\Gamma_{x x}\left(\tau_{1}-\tau_{2}\right)} \mathcal{E}\left(\tau_{2}\right) \int_{-\infty}^{\tau_{2}} d \tau_{3} e^{-\Gamma_{x}\left(\tau_{2}-\tau_{3}\right)} \mathcal{E}\left(\tau_{3}\right) \\
& \times e^{i \omega\left(t+\tau_{1}-\tau_{2}-\tau_{3}\right)}\left\langle\Omega_{0}\left|\tilde{P}(t) \tilde{P}\left(\tau_{1}\right) \tilde{P}^{\dagger}\left(\tau_{2}\right) \tilde{P}^{\dagger}\left(\tau_{3}\right)\right| \Omega_{0}\right\rangle_{\mathrm{c}} \\
&- \int_{-\infty}^{t} d \tau_{1} e^{-\Gamma_{x}\left(t-\tau_{1}\right)} \mathcal{E}\left(\tau_{1}+T\right) \int_{-\infty}^{t} d \tau_{2} e^{-\Gamma_{x x}\left(t-\tau_{2}\right)} \mathcal{E}\left(\tau_{2}\right) \int_{-\infty}^{\tau_{2}} d \tau_{3} e^{-\Gamma_{x}\left(\tau_{2}-\tau_{3}\right)} \mathcal{E}\left(\tau_{3}\right) \\
&\left.\times e^{i \omega\left(t+\tau_{1}-\tau_{2}-\tau_{3}\right)}\left\langle\Omega_{0}\left|\tilde{P}\left(\tau_{1}\right) \tilde{P}(t) \tilde{P}^{\dagger}\left(\tau_{2}\right) \tilde{P}^{\dagger}\left(\tau_{3}\right)\right| \Omega_{0}\right\rangle_{\mathrm{c}}\right\} .
\end{aligned}
$$

In most cases the four-point response functions which appear on the right-hand side of Equation 3.69 are too complicated to allow the exact calculation of the multidimensional integrals. However, if the response functions and the decay terms are slowly variable in comparison with $\mathcal{E}(t)$, the envelope function can be treated as a $\delta$ function and the integrals can be performed exactly. If the delay time $T$ is positive, the four-wave-mixing signal is then determined by the relation

$$
\begin{aligned}
P_{2 \vec{k}_{1}-\vec{k}_{2}}^{(3)}(t)= & \Theta(t) \frac{(-i)^{3}}{2^{3}} e^{-i\left(\omega T-\left(2 \vec{k}_{1}-\vec{k}_{2}\right) \vec{R}\right)} e^{-\Gamma_{x} T} \\
\times & \left\{e^{-\left\{\Gamma_{x}+\Gamma_{0}\right\} t}\left\langle\Omega_{0}\left|\tilde{P}(-T) \tilde{P}^{\dagger}(0) \tilde{P}(t) \tilde{P}^{\dagger}(0)\right| \Omega_{0}\right\rangle_{\mathrm{c}}\right. \\
& \left.-e^{-\left\{\Gamma_{x}+\Gamma_{x x}\right\} t} \frac{1}{2}\left\langle\Omega_{0}\left|\tilde{P}(-T) \tilde{P}(t) \tilde{P}^{\dagger}(0) \tilde{P}^{\dagger}(0)\right| \Omega_{0}\right\rangle_{\mathrm{c}}\right\}
\end{aligned}
$$

whereas the signal for negative delay times is given by

$$
\begin{aligned}
P_{2 \vec{k}_{1}-\vec{k}_{2}}^{(3)}(t)= & \Theta(t+T) \frac{(-i)^{3}}{2^{3}} e^{-i\left(\omega T-\left(2 \vec{k}_{1}-\vec{k}_{2}\right) \vec{R}\right)} e^{\Gamma_{x x} T} \\
\times & \left\{e^{-\Gamma_{x}(t+T)} \frac{1}{2}\left\langle\Omega_{0}\left|\tilde{P}(t) \tilde{P}(-T) \tilde{P}^{\dagger}(0) \tilde{P}^{\dagger}(0)\right| \Omega_{0}\right\rangle_{\mathrm{c}}\right. \\
& \left.-e^{-\left\{\Gamma_{x}+\Gamma_{x x}\right\}(t+T)} \frac{1}{2}\left\langle\Omega_{0}\left|\tilde{P}(-T) \tilde{P}(t) \tilde{P}^{\dagger}(0) \tilde{P}^{\dagger}(0)\right| \Omega_{0}\right\rangle_{\mathrm{c}}\right\} .
\end{aligned}
$$

Generally, the detector which registers the four-wave-mixing signal only measures the 
time-integrated intensity, which is proportional to

$$
W(T)=\lim _{L \rightarrow \infty} \frac{1}{L^{2}} \int_{-\infty}^{\infty} d t\left|P_{2 \vec{k}_{1}-\vec{k}_{2}}^{(3)}(t)\right|^{2} .
$$

The only parameter of the experiment which will be varied in the following is the delay time between the two pulses.

If the particles do not interact with each other, the exact four-point response functions can be calculated easily since a system of free conduction electrons and valence holes is only a collection of independent two-level systems with different transition energies. In the thermodynamic limit the intensity of the polarization is given by

$$
\lim _{L \rightarrow \infty} \frac{1}{L^{2}}\left|P_{2 \vec{k}_{1}-\vec{k}_{2}}^{(3)}(t)\right|^{2}=\Theta(T) \Theta(t) \frac{1}{(2 \pi)^{4}} \frac{1}{16} \frac{1}{\left(v_{f} t-v_{f} T\right)^{2}+(4 \Lambda)^{2}}
$$

and the time-integrated four-wave-mixing signal is proportional to

$$
W(T)=\Theta(T) \frac{1}{(2 \pi)^{4}} \frac{\pi}{64} \frac{1}{v_{f} \Lambda}\left\{\frac{1}{2}+\frac{1}{\pi} \arctan \left(\frac{v_{f} T}{4 \Lambda}\right)\right\},
$$

if all decay terms are neglected. It is well known that the time-integrated signal for free two-level systems vanishes completely, if the delay time is negative.

In the presence of an attractive electron-hole interaction the four-point response functions which appear in Equations 3.70 and 3.71 can be calculated in a similar way as the two-point response functions in Section 3.4. It emerges that the most important new feature which has to be taken into account is the influence of the relative distance between the two valence holes which are created by the external pulses. In contrast to that, the position of the valence hole which appears as a parameter in the expression for the two-point response function in Equation 3.50 has no impact on the properties of this response function due to the homogeneity of the external excitation. This fact can justify the description of the valence hole as an object without a structure when calculating the linear response of the system $[13,14,12,15,16]$. However, it should be kept in mind that the nonlinear response functions for such systems [60] differ essentially from the nonlinear response functions which are presented here.

Since the calculation of the four-point response functions is much more extensive than the calculation of the two-point response functions the details of this calculation are presented in Appendix C. The intensity of the four-wave-mixing signal is approximately described by the relation

$$
\begin{aligned}
\lim _{L \rightarrow \infty} \frac{1}{L^{2}}\left|P_{2 \vec{k}_{1}-\vec{k}_{2}}^{(3)}(t)\right|^{2} & \approx \frac{1}{(2 \pi)^{4}}|z|^{4}\left\{\sin ^{4}\left(\pi V_{0}\right)+4 \sin ^{2}\left(\pi\left(V_{0}+V_{0}^{2}\right)\right) \cos ^{2}\left(\pi V_{0}\right)\right\} \\
& \times\left|v_{f} T\right|^{-2(1+\alpha)}\left|v_{f} t\right|^{-2 \alpha} e^{-2 \Gamma_{x}(t+T)} e^{-2 \Gamma_{x x} t}
\end{aligned}
$$

in the thermodynamic limit, if $t \gg|T|$ and $|T| \gg x_{\tilde{v}} v_{f}^{-1}$. If $\alpha$ is negative, the time behavior of the signal intensity is determined by the diametrically opposed influences 
of the factor $t^{-2 \alpha}$, which increases algebraically, and the factor $e^{-\left\{2 \Gamma_{x}+2 \Gamma_{x x}\right\} t}$, which decreases exponentially. The latter ensures the convergence of the time integrated signal.

If it is assumed that the intensity of the four-wave-mixing signal is equal to the expression on the right-hand side of Equation 3.75, the time-integrated signal satisfies the equation

$$
\begin{aligned}
W(T) & =\frac{1}{(2 \pi)^{4}}|z|^{4}\left\{\sin ^{4}\left(\pi V_{0}\right)+4 \sin ^{2}\left(\pi\left(V_{0}+V_{0}^{2}\right)\right) \cos ^{2}\left(\pi V_{0}\right)\right\} \\
& \times\left|v_{f} T\right|^{-2(1+\alpha)}\left(2 \Gamma_{x}+2 \Gamma_{x x}\right)^{2 \alpha-1} v_{f}^{-2 \alpha} \\
& \times\left\{\Theta(T) e^{-2 \Gamma_{x} T} \Gamma(1-2 \alpha)+\Theta(-T) e^{2 \Gamma_{x x} T} \Gamma\left(1-2 \alpha,-\left(2 \Gamma_{x}+2 \Gamma_{x x}\right) T\right)\right\}
\end{aligned}
$$

where $\Gamma(\ldots, \ldots)$ denotes the incomplete gamma function [7]. Although the approximated intensity of the signal from Equation 3.75 only depends on the absolute value of the delay time $T$, the function $W(T)$ is not symmetric with respect to $T=0$. This is due to the fact that the lower boundary for the time integration in Equation 3.72 depends on the delay time $T$, if $\mathrm{T}$ is negative, while it is independent of $T$ for positive delay times. As long as $\Gamma_{x}^{-1} \gg|T|$ and $\Gamma_{x x}^{-1} \gg|T|$ the behavior of $W(T)$ is dominated by the algebraic decrease with the exponent $-2(1+\alpha)$ and the differences between positive and negative delay times can be neglected. For larger delay times, however, the influence of the exponential factors $e^{-2 \Gamma_{x} T}$ and $e^{2 \Gamma_{x x} T}$ becomes dominant.

Since the approximated expression for the time-resolved four-wave-mixing signal from Equation 3.75 should only be used, if $t$ is much larger than the absolute value of $T$, the function on the right-hand side of Equation 3.76 can only be accepted as a good approximation for the exact time-integrated signal, if the delay time is much smaller than the excitonic and biexcitonic relaxation times. In order to illustrate this statement, the complete time-resolved four-wave-mixing signal, which has been calculated with the help of Equations C.38 to C.43, is plotted for different damping constants and fixed positive and negative delay times $T$ in Figures 3.6 and 3.7. The form of the interaction potential is the same as in Section 3.4. Concerning the decay terms it is assumed that the biexcitonic damping constant $\Gamma_{x x}$ is twice as large as the excitonic damping constant $\Gamma_{x}$ [51] while the electronic damping constant $\Gamma_{0}$ is set equal to zero. Both assumptions could be modified without changing the results qualitatively. If the inequality $\Gamma_{x} T \ll 1$ holds, the time area where the signal shows a power-law increase gives the dominant contribution to the time-integrated four-wave mixing signal for the positive and the negative delay time. If the damping is stronger, the weight of the signal for the positive delay time in Figure 3.6 is shifted towards the strong resonance peak at $t=|T|$, which is already present in the case of non-interacting particles (see Equation 3.73). The weight of the signal for the negative delay time in Figure 3.7 is also shifted towards $t=|T|$, but the shift is less pronounced due to the absence of a resonance peak. In both cases the description of the time-integrated four-wave-mixing signal with the help of the approximated expression from Equation 3.76 becomes questionable 


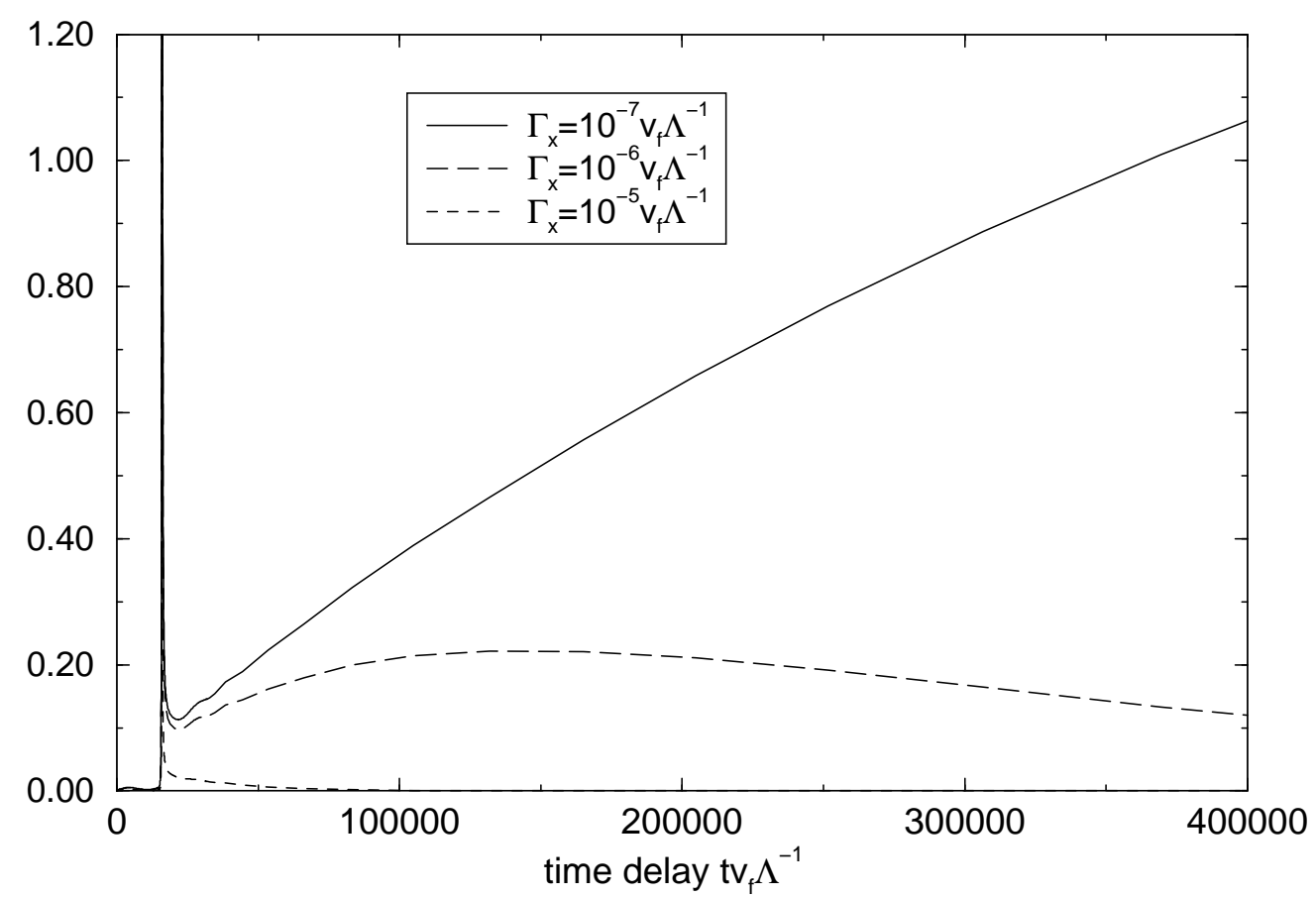

Figure 3.6: The intensity of the time-resolved four-wave-mixing signal for the delay time $T=16000 v_{f}^{-1} \Lambda$ and different excitonic damping constants $\Gamma_{x}$, the parameters for the interaction potential are $V_{0}=-0.375$ and $\lambda=1000 \Lambda$

since the signal is suppressed for $t>|T|$.

In Figure 3.8 the complete time-integrated four-wave-mixing signals are plotted for the interaction amplitude $V_{0}=-0.375$ and different damping constants. In the case of a weak damping the function is symmetric with respect to $T=0$ and its decrease for large positive and negative values is described by a power law. If the damping is stronger, the time-integrated four-wave-mixing signal for positive delay times decreases more slowly than the time-integrated signal for negative delay times since the dominant contribution to $W(T)$ for $T>0$ comes from the resonance peak mentioned above whose amplitude does not depend on the delay time $T$ in the same way as the total signal for large times $t$. If the delay time is negative, the most important contribution to the time-integrated signal still comes from the part of the signal which can be described by the approximated expression from Equation 3.75 because of the absence of a resonance peak. Consequently, the function $W(T)$ still shows a power-law decay. The total intensity, however, is reduced because of the stronger damping. If the exponential decay has suppressed the algebraic decay entirely, the typical form of the time-integrated four-wave-mixing signal is similar to the dotted curve in Figure 3.8. For negative delay times the logarithm of $W(T)$ decreases linearly with the prefactor $2 \Gamma_{x x}$. This behavior can be related to the fact that the corresponding polarization signal in Equation 3.71 contains a prefactor $e^{\Gamma_{x x} T}$. As the four-wave-mixing signal for posi- 


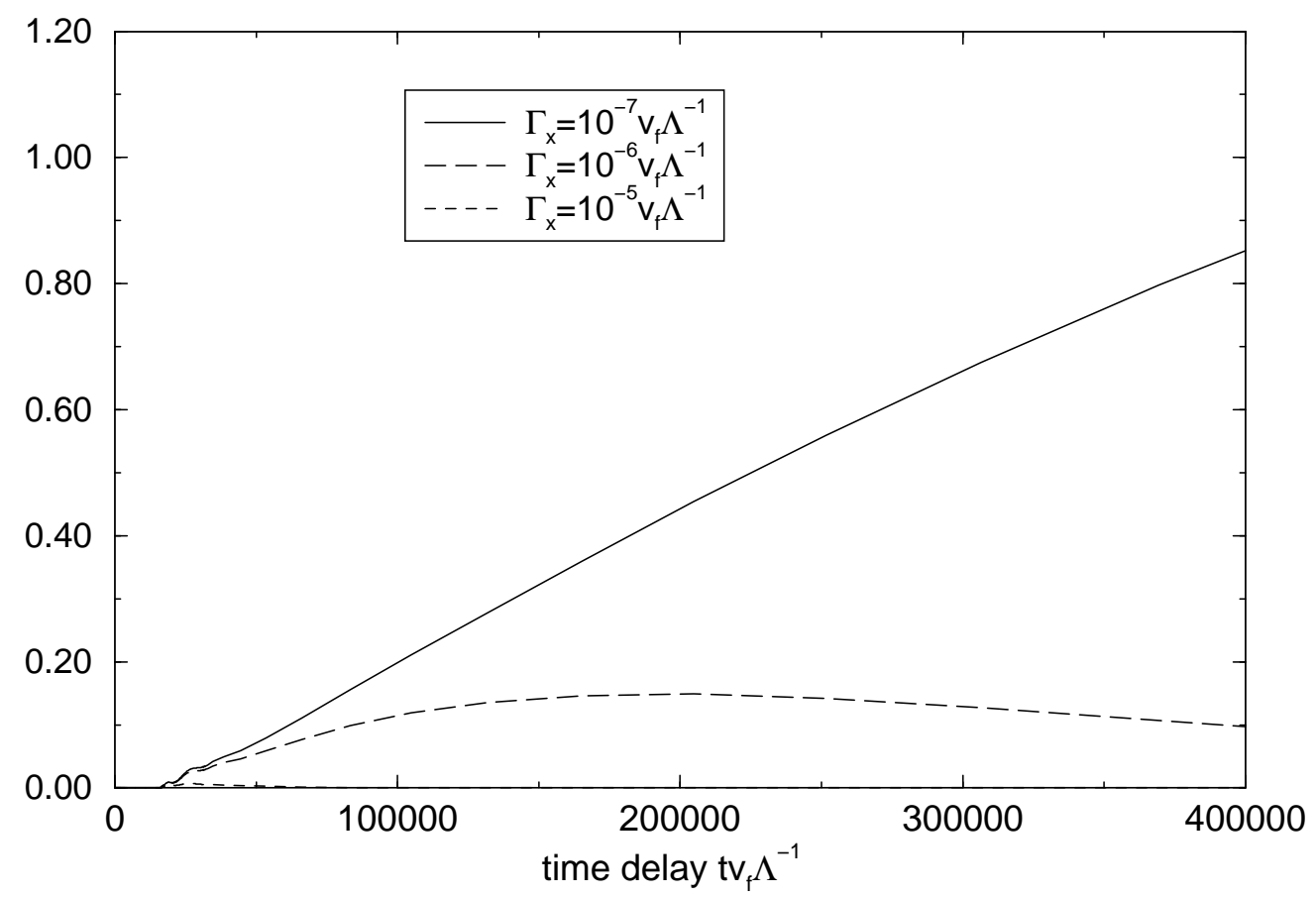

Figure 3.7: The intensity of the time-resolved four-wave-mixing signal for the delay time $T=-16000 v_{f}^{-1} \Lambda$ and different excitonic damping constants $\Gamma_{x}$, the parameters for the interaction potential are $V_{0}=-0.375$ and $\lambda=1000 \Lambda$

tive delay times in Equation 3.70 has an exponential prefactor $e^{-\Gamma_{x} T}$, the logarithm of the time integrated signal should decrease linearly with the prefactor $-2 \Gamma_{x}$. However, this behavior can only be observed for large positive delay times. For smaller positive values of $T$ the function $W(T)$ decreases like $e^{-2\left\{\Gamma_{x x}+2 \Gamma_{x}\right\} T}$. This can be put down to the fact that the dominant contribution to the time-integrated four-wave-mixing signal for small positive delay times comes from the signal in the vicinity of the resonance peak at $t=T$ whose amplitude decreases like $e^{-\left\{2 \Gamma_{x}+\Gamma_{x x}\right\} T}$.

In Figures 3.9 to 3.12 the complete time-integrated four-wave-mixing signals for different interaction amplitudes and different delay times are plotted together with the corresponding approximated curves in order to illustrate the limits of validity of the approximated expression from Equation 3.76 in the case of weak damping. Since the decay of the time-integrated signals for large positive and negative delay times can be described by a power-law, double logarithmic plots are used for the graphical representation of the data.

If the delay times are negative, the exact values for the time-integrated signals agree very well with the approximated ones (see Figures 3.9 and 3.10) although minor differences can be observed for small negative delay times. However, these deviations can be explained by the fact that $T$ was assumed to be considerably larger or smaller than zero when deriving the approximated expression for the four-wave-mixing sig- 


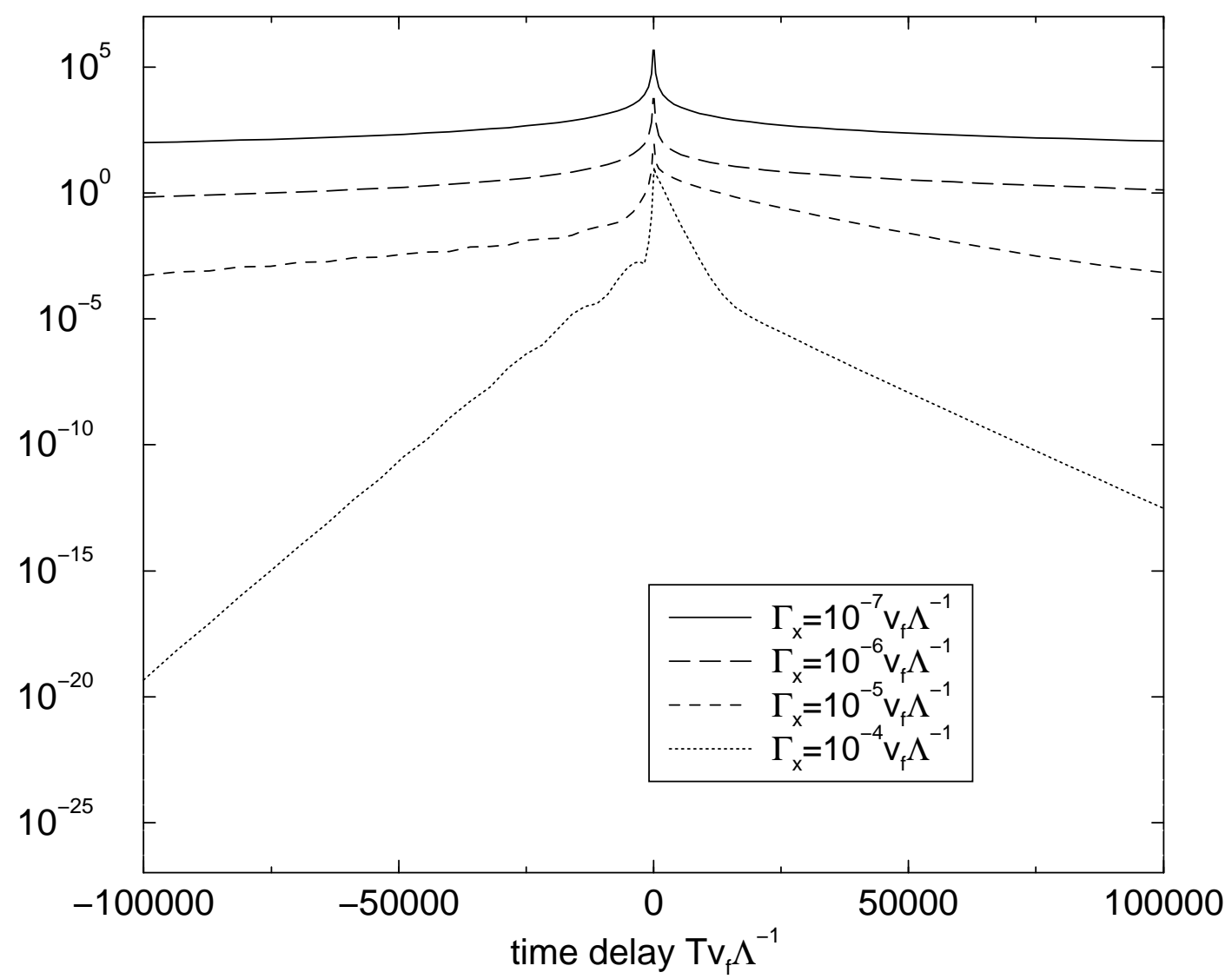

Figure 3.8: The time-integrated four-wave-mixing signals for different delay times $T$ and different excitonic damping constants $\Gamma_{x}$, the parameters for the interaction potential are $V_{0}=-0.375$ and $\lambda=1000 \Lambda$

nal. The corresponding plots for positive delay times which are depicted in Figures 3.11 and 3.12 also show a good agreement between the complete time-integrated signals and the approximated ones apart from the curves for the interaction amplitudes $V_{0}=-0.125$ and $V_{0}=-0.875$. In both cases the damping is still too strong so that the contributions to the time-integrated signals which are related to the short time dynamics of the time-resolved four-wave-mixing signals cannot be neglected. 


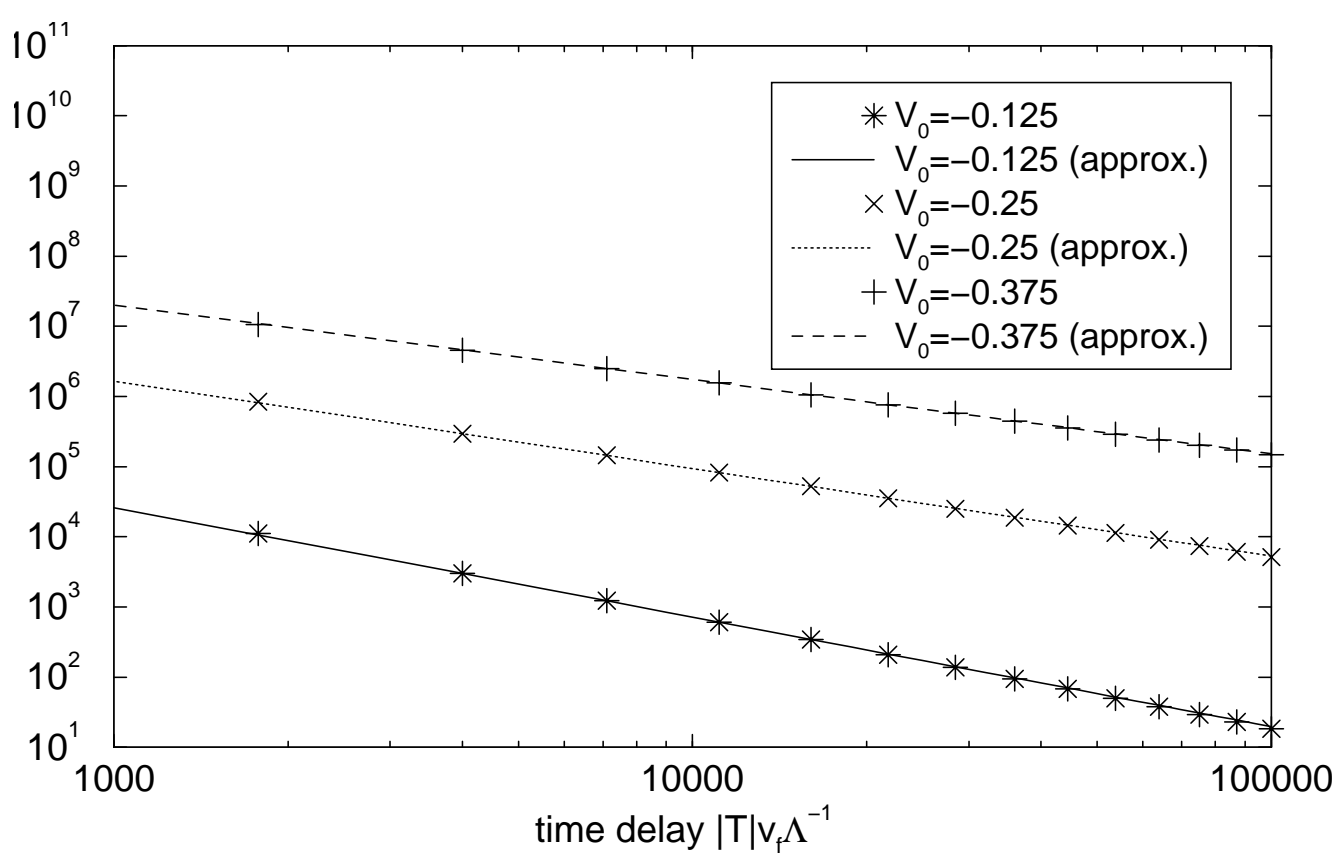

Figure 3.9: The time-integrated four-wave-mixing signals for different negative delay times $T$ and different interaction amplitudes $V_{0}$ together with the corresponding approximated curves, $\Gamma_{x}=8 \times 10^{-9} v_{f} \Lambda^{-1}, \lambda=1000 \Lambda$

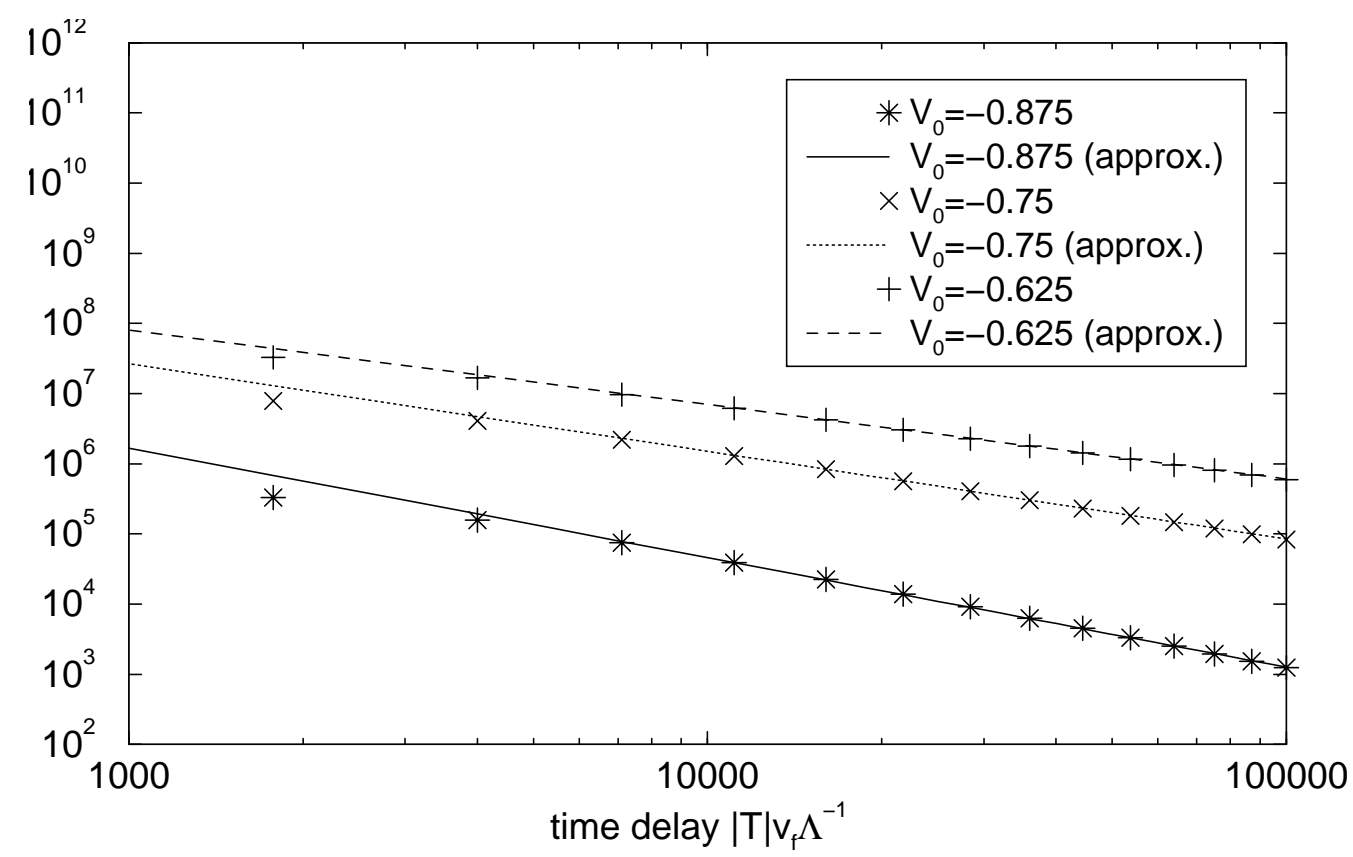

Figure 3.10: The time-integrated four-wave-mixing signals for different negative delay times $T$ and different interaction amplitudes $V_{0}$ together with the corresponding approximated curves, $\Gamma_{x}=8 \times 10^{-9} v_{f} \Lambda^{-1}, \lambda=1000 \Lambda$ 


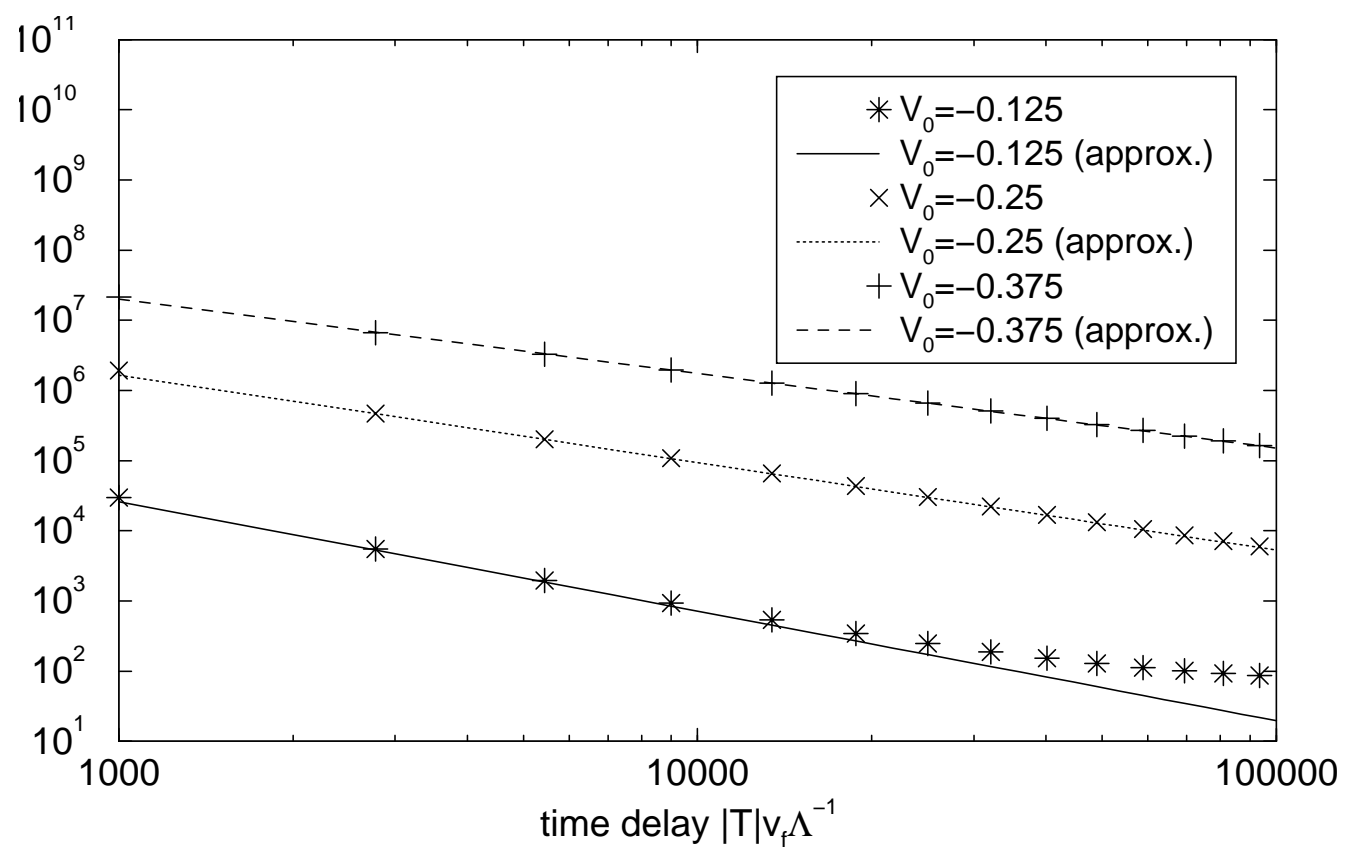

Figure 3.11: The time-integrated four-wave-mixing signals for different positive delay times $T$ and different interaction amplitudes $V_{0}$ together with the corresponding approximated curves, $\Gamma_{x}=8 \times 10^{-9} v_{f} \Lambda^{-1}, \lambda=1000 \Lambda$

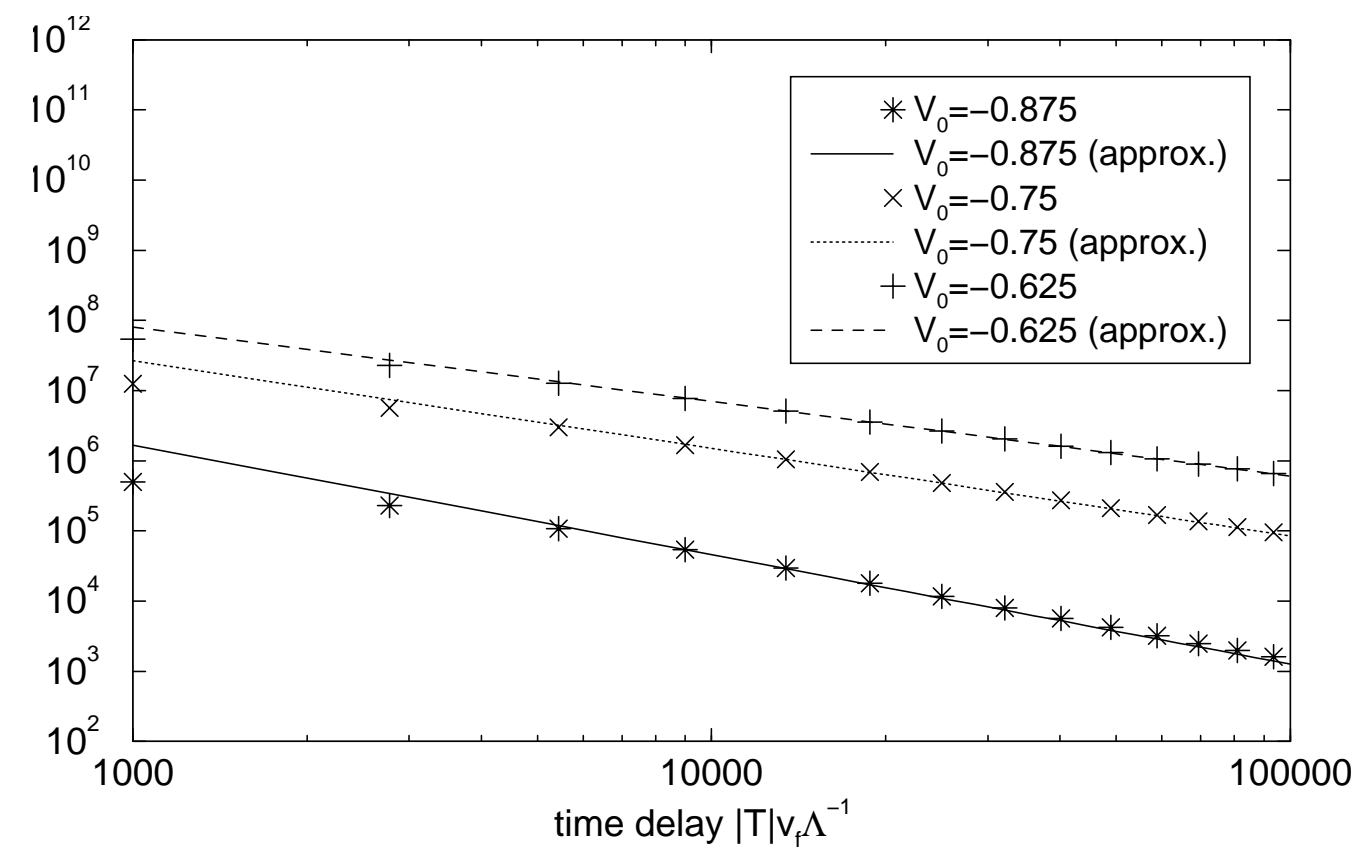

Figure 3.12: The time-integrated four-wave-mixing signals for different positive delay times $T$ and different interaction amplitudes $V_{0}$ together with the corresponding approximated curves, $\Gamma_{x}=8 \times 10^{-9} v_{f} \Lambda^{-1}, \lambda=1000 \Lambda$ 


\section{Chapter 4}

\section{Summary and Outlook}

In Chapter 2, the relaxation behavior of optically excited electron-hole pairs in a onedimensional semiconductor, which are coupled to longitudinal optical phonons with an initial lattice temperature $T \geq 0$, was studied with the help of quantum kinetic equations. They were derived within the framework of the density matrix formalism in the self-consistent Born approximation of second order (with respect to the electron-phonon interaction). Apart from Hartree-Fock-like Coulomb contributions, these equations contain additional Coulomb terms, the so-called vertex corrections, by which the influence of the electron-electron interaction on the electron-phonon scattering processes is taken into account. An alternative system of kinetic equations was derived with the help of non-equilibrium Green's functions following the presentations which are found in the literature [43]. A comparison showed that the kinetic equations which are formulated within the framework of the non-equilibrium Green's function formalism do not include the vertex corrections mentioned above.

The additional Coulomb terms are essential for a correct description of excitons with finite center-of-mass momenta. This became clear when the imaginary part of the linear susceptibility for excitonic excitations was calculated neglecting the influence of the optically excited continuum states. When the vertex corrections were taken into account, the imaginary part of the susceptibility was dominated by one resonance peak whose width increased with temperature. When the vertex corrections were neglected the width of this resonance peak became considerably broader, and it was possible to observe the formation of a double peak structure with increasing temperature.

The differences between the results can be traced to the fact that the form of the susceptibility function in the vicinity of the excitonic resonance is predominantly influenced by phonon absorption processes. The two systems of kinetic equations provide different descriptions of the energy spectra of electron-hole pairs after these absorption processes in which the pairs, whose initial center-of-mass momenta after the optical excitation are always zero, assume finite center-of-mass momenta. If the linear dynamics is described with the help of the kinetic equations without vertex corrections, these electron-hole pairs are treated as free particles. In contrast to that, the kinetic

equations, which include the vertex corrections, allow the complete description of the 
interacting electron-hole pairs after the phonon absorption.

For comparison, the linear susceptibility functions for continuum excitations were calculated using the quantum kinetic equations with vertex corrections. They exhibited no significant differences from the susceptibility functions for non-interacting electron-hole pairs. This indicates that all Coulomb terms have only a minor impact on the dynamics of unbound electron-hole pairs in the model considered here.

The results of the investigation of the linear susceptibility were confirmed by calculating the time behavior of electron and phonon densities for different detunings in the weak nonlinear regime. When the excitation was tuned to the exciton resonance, the additional Coulomb terms led to qualitative changes of the time behavior in comparison with the results which were obtained within the framework of the ordinary Hartree-Fock approximation. For continuum excitations, however, the additional terms had no significant influence on the time behavior in accordance with the corresponding results for the linear response. The nonlinear dynamics for excitonic excitations was also calculated using a simplified version of the kinetic equations with vertex corrections which had been adapted to the situation in the weak nonlinear regime. As expected, these equations gave similar results as the kinetic equations with the complete vertex corrections.

The numerical studies of the linear and nonlinear response indicate that the vertex corrections have to be included in the kinetic equations if the time behavior of the system is dominated by the excitonic excitations. On the other hand, it seems justified to neglect their influence when describing continuum excitations, at least in the weak nonlinear regime. Independent of that kind of optical excitation, however, it is always necessary to take the vertex corrections into account if one intends to describe the excitonic effects in a consistent way. It seems justified to assume that the results for the one-dimensional semiconductor form a good basis for further studies, which could, for example, deal with the role of the vertex corrections in the quantum kinetic equations for higher dimensional systems.

The linear and nonlinear optical response of a one-dimensional n-doped semiconductor with two bands was studied in Chapter 3. While the energy dispersion of the valence band was chosen to be constant, the dispersion of the conduction band was linearized with respect to the two Fermi points. In the calculations, only the attractive interaction between the conduction electrons and the valence holes was taken into account, whereas the repulsive interaction between the conduction electrons was neglected in accordance with the approaches found in the literature $[15,19]$. The introduction of the linearized dispersion for the conduction electrons made it possible to describe the eigenstates and eigenenergies of the free conduction electrons within the framework of a bosonic representation. Within this bosonic representation, it was also possible to describe the conduction electrons in the presence of the attractive potential of one or several valence holes by mapping the Hamiltonian of the interacting system on the free Hamiltonian with the help of a unitary transformation.

The optical response of the many-body system to the influence of an external electromagnetic field was then calculated by expanding the total polarization in a power 
series with respect to the intensity of the external field. The expressions for the linear contribution and the first nonlinear contribution, which is proportional to the third power of the external field, were given explicitly. The influence of dissipative processes was taken into account by introducing phenomenological damping constants which ensured the convergence of the signals in the large-time limit.

When studying the linear response, attention was focused on the characteristics of the absorption spectrum, which could be calculated exactly within the framework of the bosonic representation. The calculations showed that the absorption spectrum in the vicinity of the Fermi-edge is dominated by an asymmetric algebraic singularity providing that the strength of the electron-hole interaction does not exceed a critical value. These results are in good agreement with the results presented in the literature [15].

The exact third-order response functions were calculated in a similar way as the linear response functions. They were used for the description of a degenerate four-wavemixing experiment with two excitation pulses. When calculating the time-integrated four-wave-mixing signal for a specific propagation direction as a function of the delay time between the pulses, $T$, it emerged that the decay of the time-integrated signal is symmetric with respect to $T=0$ and can approximately be described by a power law providing that the delay times are small in comparison with the decay times. As it turned out, the exponent which describes the algebraic decay of the time-integrated signal is functionally dependent on the exponent of the algebraic singularity in the linear absorption spectrum reflecting the common origin of the different phenomena. If the delay times are of the same order of magnitude as the life-times of the optical excitations, the picture changes considerably and the decay of the time-integrated signal is dominated by an exponential damping.

The investigation of the optical response of the exactly solvable one-dimensional model showed that the well-known characteristic features of the linear response functions can directly be related to features of the nonlinear response functions. Based on the results of Chapter 3, further studies can now deal with various subjects. For example, one could calculate the response functions with the help of approximation methods [35, 44, 59] and compare them with the corresponding exact expressions in order to test the quality of the approximations. One could also study if the nonlinear time behavior of higher dimensional models shows similar features as the dynamics of the one-dimensional model presented here. Finally, the dissipative processes could be incorporated in a way which goes beyond the phenomenological description which was used in this work. 


\section{Appendix A}

\section{Kinetic Equations of Higher Order}

The calculation of the correction terms in Section 2.3 requires the knowledge of the kinetic equations for the correlated parts of the second order phonon-assisted densities from Equations 2.25 and 2.26. They are given by

$$
\begin{aligned}
& i \frac{d}{d t}\left\langle\psi_{\lambda_{1} l+p+p^{\prime}}^{\dagger} \psi_{\lambda_{2} l} b_{p} b_{p^{\prime}}\right\rangle_{t}^{c}=\left\{\omega_{p^{\prime}}+\omega_{p}\right\}\left\langle\psi_{\lambda_{1} l+p+p^{\prime}}^{\dagger} \psi_{\lambda_{2} l} b_{p} b_{p^{\prime}}\right\rangle_{t}^{c} \\
& +\sum_{\sigma}\left\{\tilde{\varepsilon}_{\lambda_{2} \sigma ; l}(t)\left\langle\psi_{\lambda_{1} l+p+p^{\prime}}^{\dagger} \psi_{\sigma l} b_{p} b_{p^{\prime}}\right\rangle_{t}^{c}-\tilde{\varepsilon}_{\sigma \lambda_{1} ; l+p+p^{\prime}}(t)\left\langle\psi_{\sigma l+p+p^{\prime}}^{\dagger} \psi_{\lambda_{2} l} b_{p} b_{p^{\prime}}\right\rangle_{t}^{c}\right\} \\
& +\delta\left\langle\psi_{\lambda_{1} l+p+p^{\prime}}^{\dagger} \psi_{\lambda_{2} l} b_{p} b_{p^{\prime}}\right\rangle_{e p}^{c}+\delta\left\langle\psi_{\lambda_{1} l+p+p^{\prime}}^{\dagger} \psi_{\lambda_{2} l} b_{p} b_{p^{\prime}}\right\rangle_{e e}^{c}
\end{aligned}
$$

and

$$
\begin{aligned}
& i \frac{d}{d t}\left\langle\psi_{\lambda_{1} l+p^{\prime}-p}^{\dagger} \psi_{\lambda_{2} l} b_{p}^{\dagger} b_{p^{\prime}}\right\rangle_{t}^{c}=\left\{\omega_{p^{\prime}}-\omega_{p}\right\}\left\langle\psi_{\lambda_{1} l+p^{\prime}-p}^{\dagger} \psi_{\lambda_{2} l} b_{p}^{\dagger} b_{p^{\prime}}\right\rangle_{t}^{c} \\
& +\sum_{\sigma}\left\{\tilde{\varepsilon}_{\lambda_{2} \sigma ; l}(t)\left\langle\psi_{\lambda_{1} l+p^{\prime}-p}^{\dagger} \psi_{\sigma l} b_{p}^{\dagger} b_{p^{\prime}}\right\rangle_{t}^{c}-\tilde{\varepsilon}_{\sigma \lambda_{1} ; l+p^{\prime}-p}(t)\left\langle\psi_{\sigma l+p^{\prime}-p}^{\dagger} \psi_{\lambda_{2} l} b_{p}^{\dagger} b_{p^{\prime}}\right\rangle_{t}^{c}\right\} \\
& +\delta\left\langle\psi_{\lambda_{1} l+p^{\prime}-p}^{\dagger} \psi_{\lambda_{2} l} b_{p}^{\dagger} b_{p^{\prime}}\right\rangle_{e p}^{c}+\delta\left\langle\psi_{\lambda_{1} l+p^{\prime}-p}^{\dagger} \psi_{\lambda_{2} l} b_{p}^{\dagger} b_{p^{\prime}}\right\rangle_{e e}^{c}
\end{aligned}
$$

if the different contributions are arranged in the same way as in the case of the kinetic equations for first order phonon-assisted densities. The collision terms are defined in Equations A.3 to A.6 (see below).

When deriving these equations it has to be taken into account that the expressions on the right-hand sides of Equations A.1 and A.2 do not contain all terms which appear in the corresponding expressions for the complete second order phonon-assisted densities after a factorization of the density functions. This is due to the fact that the latter also contain terms which coincide with terms which appear in the kinetic equations for the products of electron and phonon densities on the right-hand sides of Equations 2.25 and 2.26 .

This phenomenon is characteristic for the kinetic equations of all correlation functions which are not equal to their counterparts among the complete density functions. 
In Reference [42] it was interpreted as a consequence of the so-called linked-cluster theorem.

The explicit form of the two collision terms which describe the influence of the electron-phonon scattering processes is determined by the equations

$$
\begin{aligned}
& \left.\delta \psi_{\lambda_{1} l+p+p^{\prime}}^{\dagger} \psi_{\lambda_{2} l} b_{p} b_{p^{\prime}}\right\rangle_{e p}^{c}=g_{p^{\prime}}^{\lambda_{2}}\left\langle\psi_{\lambda_{1} l+p+p^{\prime}}^{\dagger} \psi_{\lambda_{2} l+p^{\prime}} b_{p}\right\rangle_{t}+g_{p}^{\lambda_{2}}\left\langle\psi_{\lambda_{1} l+p+p^{\prime}}^{\dagger} \psi_{\lambda_{2} l+p} b_{p^{\prime}}\right\rangle_{t} \\
& +\left\{g_{p^{\prime}}^{\lambda_{2}}\left\langle\psi_{\lambda_{1} l+p+p^{\prime}}^{\dagger} \psi_{\lambda_{2} l+p^{\prime}} b_{p}\right\rangle_{t}-g_{p^{\prime}}^{\lambda_{1}}\left\langle\psi_{\lambda_{1} l+p}^{\dagger} \psi_{\lambda_{2} l} b_{p}\right\rangle_{t}\right\}\left\{\left\langle b_{-p^{\prime}} b_{p^{\prime}}\right\rangle_{t}+\left\langle b_{p^{\prime}}^{\dagger} b_{p^{\prime}}\right\rangle_{t}\right\} \\
& +\left\{g_{p}^{\lambda_{2}}\left\langle\psi_{\lambda_{1} l+p+p^{\prime}}^{\dagger} \psi_{\lambda_{2} l+p} b_{p^{\prime}}\right\rangle_{t}-g_{p}^{\lambda_{1}}\left\langle\psi_{\lambda_{1} l+p^{\prime}}^{\dagger} \psi_{\lambda_{2} l} b_{p^{\prime}}\right\rangle_{t}\right\}\left\{\left\langle b_{-p} b_{p}\right\rangle_{t}+\left\langle b_{p}^{\dagger} b_{p}\right\rangle_{t}\right\} \\
& -\sum_{\sigma} g_{p^{\prime}}^{\sigma}\left\{\left\langle\psi_{\lambda_{1} l+p+p^{\prime}}^{\dagger} \psi_{\sigma l+p+p^{\prime}}\right\rangle_{t}\left\langle\psi_{\sigma l+p}^{\dagger} \psi_{\lambda_{2} l} b_{p}\right\rangle_{t}+\left\langle\psi_{\lambda_{1} l+p+p^{\prime}}^{\dagger} \psi_{\sigma l+p^{\prime}} b_{p}\right\rangle_{t}\left\langle\psi_{\sigma l}^{\dagger} \psi_{\lambda_{2} l}\right\rangle_{t}\right\} \\
& -\sum_{\sigma} g_{p}^{\sigma}\left\{\left\langle\psi_{\lambda_{1} l+p+p^{\prime}}^{\dagger} \psi_{\sigma l+p+p^{\prime}}\right\rangle_{t}\left\langle\psi_{\sigma l+p^{\prime}}^{\dagger} \psi_{\lambda_{2} l} b_{p^{\prime}}\right\rangle_{t}+\left\langle\psi_{\lambda_{1} l+p+p^{\prime}}^{\dagger} \psi_{\sigma l+p} b_{p^{\prime}}\right\rangle_{t}\left\langle\psi_{\sigma l}^{\dagger} \psi_{\lambda_{2} l}\right\rangle_{t}\right\} \\
& +\left\{g_{p+p^{\prime}}^{\lambda_{2}}\left\langle\psi_{\lambda_{1} l+p+p^{\prime}}^{\dagger} \psi_{\lambda_{2} l+p+p^{\prime}}\right\rangle_{t}-g_{p+p^{\prime}}^{\lambda_{1}}\left\langle\psi_{\lambda_{1} l}^{\dagger} \psi_{\lambda_{2} l}\right\rangle_{t}\right\}\left\{\left\langle b_{-p-p^{\prime}} b_{p} b_{p^{\prime}}\right\rangle_{t}+\left\langle b_{p+p^{\prime}}^{\dagger} b_{p} b_{p^{\prime}}\right\rangle_{t}\right\} \\
& +\sum_{q \neq 0} g_{q}^{\lambda_{2}}\left\{\left\langle\psi_{\lambda_{1} l+p+p^{\prime}}^{\dagger} \psi_{\lambda_{2} l-q} b_{q} b_{p} b_{p^{\prime}}\right\rangle_{t}^{c}+\left\langle\psi_{\lambda_{1} l+p+p^{\prime}}^{\dagger} \psi_{\lambda_{2} l+q} b_{q}^{\dagger} b_{p} b_{p^{\prime}}\right\rangle_{t}^{c}\right\} \\
& -\sum_{q \neq 0} g_{q}^{\lambda_{1}}\left\{\left\langle\psi_{\lambda_{1} l+p+p^{\prime}+q}^{\dagger} \psi_{\lambda_{2} l} b_{q} b_{p} b_{p^{\prime}}\right\rangle_{t}^{c}+\left\langle\psi_{\lambda_{1} l+p+p^{\prime}-q}^{\dagger} \psi_{\lambda_{2} l} b_{q}^{\dagger} b_{p} b_{p^{\prime}}\right\rangle_{t}^{c}\right\} \\
& +\sum_{\sigma k} g_{p^{\prime}}^{\sigma}\left\langle\psi_{\lambda_{1} l+p+p^{\prime}}^{\dagger} \psi_{\sigma k}^{\dagger} \psi_{\sigma k+p^{\prime}} \psi_{\lambda_{2} l} b_{p}\right\rangle_{t}^{c}+\sum_{\sigma k} g_{p}^{\sigma}\left\langle\psi_{\lambda_{1} l+p+p^{\prime}}^{\dagger} \psi_{\sigma k}^{\dagger} \psi_{\sigma k+p} \psi_{\lambda_{2} l} b_{p^{\prime}}\right\rangle_{t}^{c} \quad \text { (A.3) }
\end{aligned}
$$

and

$$
\begin{aligned}
& \delta\left\langle\psi_{\lambda_{1} l+p^{\prime}-p}^{\dagger} \psi_{\lambda_{2} l} b_{p}^{\dagger} b_{p^{\prime}}\right\rangle_{e p}^{c}=g_{p^{\prime}}^{\lambda_{2}}\left\langle\psi_{\lambda_{1} l+p^{\prime}-p}^{\dagger} \psi_{\lambda_{2} l+p^{\prime}} b_{p}^{\dagger}\right\rangle_{t}-g_{p}^{\lambda_{1}}\left\langle\psi_{\lambda_{1} l+p^{\prime}}^{\dagger} \psi_{\lambda_{2} l} b_{p^{\prime}}\right\rangle_{t} \\
& +\left\{g_{p^{\prime}}^{\lambda_{2}}\left\langle\psi_{\lambda_{1} l+p^{\prime}-p}^{\dagger} \psi_{\lambda_{2} l+p^{\prime}} b_{p}^{\dagger}\right\rangle_{t}-g_{p^{\prime}}^{\lambda_{1}}\left\langle\psi_{\lambda_{1} l-p}^{\dagger} \psi_{\lambda_{2} l} b_{p}^{\dagger}\right\rangle_{t}\right\}\left\{\left\langle b_{-p^{\prime}} b_{p^{\prime}}\right\rangle_{t}+\left\langle b_{p^{\prime}}^{\dagger} b_{p^{\prime}}\right\rangle_{t}\right\} \\
& +\left\{g_{p}^{\lambda_{2}}\left\langle\psi_{\lambda_{1} l+p^{\prime}-p}^{\dagger} \psi_{\lambda_{2} l-p} b_{p^{\prime}}\right\rangle_{t}-g_{p}^{\lambda_{1}}\left\langle\psi_{\lambda_{1} l+p^{\prime}}^{\dagger} \psi_{\lambda_{2} l} b_{p^{\prime}}\right\rangle_{t}\right\}\left\{\left\langle b_{p}^{\dagger} b_{-p}^{\dagger}\right\rangle_{t}+\left\langle b_{p}^{\dagger} b_{p}\right\rangle_{t}\right\} \\
& -\sum_{\sigma} g_{p^{\prime}}^{\sigma}\left\{\left\langle\psi_{\lambda_{1} l+p^{\prime}-p}^{\dagger} \psi_{\sigma l+p^{\prime}-p}\right\rangle_{t}\left\langle\psi_{\sigma l-p}^{\dagger} \psi_{\lambda_{2} l} b_{p}^{\dagger}\right\rangle_{t}+\left\langle\psi_{\lambda_{1} l+p^{\prime}-p}^{\dagger} \psi_{\sigma l+p^{\prime}} b_{p}^{\dagger}\right\rangle_{t}\left\langle\psi_{\sigma l}^{\dagger} \psi_{\lambda_{2} l}\right\rangle_{t}\right\} \\
& +\sum_{\sigma} g_{p}^{\sigma}\left\{\left\langle\psi_{\lambda_{1} l+p^{\prime}-p}^{\dagger} \psi_{\sigma l+p^{\prime}-p}\right\rangle_{t}\left\langle\psi_{\sigma l+p^{\prime}}^{\dagger} \psi_{\lambda_{2} l} b_{p^{\prime}}\right\rangle_{t}+\left\langle\psi_{\lambda_{1} l+p^{\prime}-p}^{\dagger} \psi_{\sigma l-p} b_{p^{\prime}}\right\rangle_{t}\left\langle\psi_{\sigma l}^{\dagger} \psi_{\lambda_{2} l}\right\rangle_{t}\right\} \\
& +\left\{g_{p-p^{\prime}}^{\lambda_{2}}\left\langle\psi_{\lambda_{1} l+p^{\prime}-p}^{\dagger} \psi_{\lambda_{2} l+p^{\prime}-p}\right\rangle_{t}-g_{p-p^{\prime}}^{\lambda_{1}}\left\langle\psi_{\lambda_{1} l}^{\dagger} \psi_{\lambda_{2} l}\right\rangle_{t}\right\}\left\{\left\langle b_{p}^{\dagger} b_{p^{\prime}} b_{p-p^{\prime}}\right\rangle_{t}+\left\langle b_{p^{\prime}-p}^{\dagger} b_{p}^{\dagger} b_{p^{\prime}}\right\rangle_{t}\right\} \\
& +\sum_{q \neq 0} g_{q}^{\lambda_{2}}\left\{\left\langle\psi_{\lambda_{1} l+p^{\prime}-p}^{\dagger} \psi_{\lambda_{2} l-q} b_{p}^{\dagger} b_{p^{\prime}} b_{q}\right\rangle_{t}^{c}+\left\langle\psi_{\lambda_{1} l+p^{\prime}-p}^{\dagger} \psi_{\lambda_{2} l+q} b_{q}^{\dagger} b_{p}^{\dagger} b_{p^{\prime}}\right\rangle_{t}^{c}\right\} \\
& -\sum_{q \neq 0} g_{q}^{\lambda_{1}}\left\{\left\langle\psi_{\lambda_{1} l+p^{\prime}-p+q}^{\dagger} \psi_{\lambda_{2} l} b_{p}^{\dagger} b_{p^{\prime}} b_{q}\right\rangle_{t}^{c}+\left\langle\psi_{\lambda_{1} l+p^{\prime}-p-q}^{\dagger} \psi_{\lambda_{2} l} b_{q}^{\dagger} b_{p}^{\dagger} b_{p^{\prime}}\right\rangle_{t}^{c}\right\} \\
& +\sum_{\sigma k} g_{p^{\prime}}^{\sigma}\left\langle\psi_{\lambda_{1} l+p^{\prime}-p}^{\dagger} \psi_{\sigma k}^{\dagger} \psi_{\sigma k+p^{\prime}} \psi_{\lambda_{2} l} b_{p}^{\dagger}\right\rangle_{t}^{c}-\sum_{\sigma k} g_{p}^{\sigma}\left\langle\psi_{\lambda_{1} l+p^{\prime}-p}^{\dagger} \psi_{\sigma k+p}^{\dagger} \psi_{\sigma k} \psi_{\lambda_{2} l} b_{p^{\prime}}\right\rangle_{t}^{c} .
\end{aligned}
$$


The sole contributions which are necessary for the calculation of the correction terms $\delta \epsilon_{c ; l}$ and $\delta \epsilon_{v ; l}$ are those which are proportional to the first order phonon-assisted densities. Therefore only the functions in the first five line of Equations A.3 and A.4 have to be considered. The remaining terms would become important, if the influence of higher order corrections with respect to the phonon-coupling constant $g$ was taken into account.

The second collision term from Equation A.1, which satisfies the relation

$$
\begin{aligned}
& \delta\left\langle\psi_{\lambda_{1} l+p+p^{\prime}}^{\dagger} \psi_{\lambda_{2} l} b_{p} b_{p^{\prime}}\right\rangle_{e e}^{c}=\sum_{\sigma k} V_{\lambda_{2} \sigma}\left(\left|p+p^{\prime}\right|\right)\left\langle\psi_{\lambda_{1} l+p+p^{\prime}}^{\dagger} \psi_{\lambda_{2} l+p+p^{\prime}}\right\rangle_{t}\left\langle\psi_{\sigma k+p+p^{\prime}}^{\dagger} \psi_{\sigma k} b_{p} b_{p^{\prime}}\right\rangle_{t}^{c} \\
& -\sum_{\sigma k} V_{\sigma \lambda_{1}}\left(\left|p+p^{\prime}\right|\right)\left\langle\psi_{\lambda_{1} l}^{\dagger} \psi_{\lambda_{2} l}\right\rangle_{t}\left\langle\psi_{\sigma k+p+p^{\prime}}^{\dagger} \psi_{\sigma k} b_{p} b_{p^{\prime}}\right\rangle_{t}^{c} \\
& -\sum_{\sigma k} V_{\lambda_{2} \sigma}(|l-k|)\left\langle\psi_{\lambda_{1} l+p+p^{\prime}}^{\dagger} \psi_{\sigma l+p+p^{\prime}}\right\rangle_{t}\left\langle\psi_{\sigma k+p+p^{\prime}}^{\dagger} \psi_{\lambda_{2} k} b_{p} b_{p^{\prime}}\right\rangle_{t}^{c} \\
& +\sum_{\sigma k} V_{\sigma \lambda_{1}}(|l-k|)\left\langle\psi_{\sigma l}^{\dagger} \psi_{\lambda_{2} l}\right\rangle_{t}\left\langle\psi_{\lambda_{1} k+p+p^{\prime}}^{\dagger} \psi_{\sigma k} b_{p} b_{p^{\prime}}\right\rangle_{t}^{c} \\
& +\sum_{\sigma k}\left\{V_{\lambda_{2} \sigma}\left(\left|p^{\prime}\right|\right)\left\langle\psi_{\lambda_{1} l+p+p^{\prime}}^{\dagger} \psi_{\lambda_{2} l+p^{\prime}} b_{p}\right\rangle_{t}-V_{\sigma \lambda_{1}}\left(\left|p^{\prime}\right|\right)\left\langle\psi_{\lambda_{1} l+p}^{\dagger} \psi_{\lambda_{2} l} b_{p}\right\rangle_{t}\right\}\left\langle\psi_{\sigma k+p^{\prime}}^{\dagger} \psi_{\sigma k} b_{p^{\prime}}\right\rangle_{t} \\
& +\sum_{\sigma k}\left\{V_{\lambda_{2} \sigma}(|p|)\left\langle\psi_{\lambda_{1} l+p+p^{\prime}}^{\dagger} \psi_{\lambda_{2} l+p} b_{p^{\prime}}\right\rangle_{t}-V_{\sigma \lambda_{1}}(|p|)\left\langle\psi_{\lambda_{1} l+p^{\prime}}^{\dagger} \psi_{\lambda_{2} l} b_{p^{\prime}}\right\rangle_{t}\right\}\left\langle\psi_{\sigma k+p}^{\dagger} \psi_{\sigma k} b_{p}\right\rangle_{t} \\
& -\sum_{\sigma k} V_{\lambda_{2} \sigma}(|l-k|)\left\langle\psi_{\lambda_{1} l+p+p^{\prime}}^{\dagger} \psi_{\sigma l+p^{\prime}} b_{p}\right\rangle_{t}\left\langle\psi_{\sigma k+p^{\prime}}^{\dagger} \psi_{\lambda_{2} k} b_{p^{\prime}}\right\rangle_{t} \\
& +\sum_{\sigma k} V_{\sigma \lambda_{1}}\left(\left|l+p^{\prime}-k\right|\right)\left\langle\psi_{\lambda_{1} k+p}^{\dagger} \psi_{\sigma k} b_{p}\right\rangle_{t}\left\langle\psi_{\sigma l+p^{\prime}}^{\dagger} \psi_{\lambda_{2} l} b_{p^{\prime}}\right\rangle_{t} \\
& -\sum_{\sigma k} V_{\lambda_{2} \sigma}(|l-k|)\left\langle\psi_{\lambda_{1} l+p+p^{\prime}}^{\dagger} \psi_{\sigma l+p} b_{p^{\prime}}\right\rangle_{t}\left\langle\psi_{\sigma k+p}^{\dagger} \psi_{\lambda_{2} k} b_{p}\right\rangle_{t} \\
& +\sum_{\sigma k} V_{\sigma \lambda_{1}}(|l+p-k|)\left\langle\psi_{\lambda_{1} k+p^{\prime}}^{\dagger} \psi_{\sigma k} b_{p^{\prime}}\right\rangle_{t}\left\langle\psi_{\sigma l+p}^{\dagger} \psi_{\lambda_{2} l} b_{p}\right\rangle_{t} \\
& +\sum_{\sigma k} \sum_{q} V_{\lambda_{2} \sigma}(|q|)\left\langle\psi_{\lambda_{1} l+p+p^{\prime}}^{\dagger} \psi_{\sigma k}^{\dagger} \psi_{\sigma k+q} \psi_{\lambda_{2} l-q} b_{p} b_{p^{\prime}}\right\rangle_{t}^{c} \\
& -\sum_{\sigma k} \sum_{q} V_{\sigma \lambda_{1}}(|q|)\left\langle\psi_{\lambda_{1} l+p+p^{\prime}+q}^{\dagger} \psi_{\sigma k-q}^{\dagger} \psi_{\sigma k} \psi_{\lambda_{2} l} b_{p} b_{p^{\prime}}\right\rangle_{t}^{c},
\end{aligned}
$$

describes those contributions of the electron-electron scattering processes to the dynamics of $\left\langle\psi_{\lambda_{1} l+p+p^{\prime}}^{\dagger} \psi_{\lambda_{2} l} b_{p} b_{p^{\prime}}\right\rangle^{c}$ which cannot be written as corrections to the oneparticle energy matrix. When deriving the correction terms $\delta \epsilon_{\lambda ; l}$ these contributions are neglected completely together with the dynamical Hartree-Fock contributions. In order to describe at least the excitonic effects correctly, it would be necessary to consider the terms in the first four lines which bear resemblance to the terms in the first four lines of Equation 2.24. This observation also applies to the corresponding collision term for the second order phonon-assisted density function $\left\langle\psi_{\lambda_{1} l+p^{\prime}-p}^{\dagger} \psi_{\lambda_{2} l} b_{p}^{\dagger} b_{p^{\prime}}\right\rangle^{c}$, 
which is defined by the equation

$$
\begin{aligned}
& \delta\left\langle\psi_{\lambda_{1} l+p^{\prime}-p}^{\dagger} \psi_{\lambda_{2} l} b_{p}^{\dagger} b_{p^{\prime}}\right\rangle_{e e}^{c}=\sum_{\sigma k} V_{\lambda_{2} \sigma}\left(\left|p^{\prime}-p\right|\right)\left\langle\psi_{\lambda_{1} l+p^{\prime}-p}^{\dagger} \psi_{\lambda_{2} l+p^{\prime}-p}\right\rangle_{t}\left\langle\psi_{\sigma k+p^{\prime}-p}^{\dagger} \psi_{\sigma k} b_{p}^{\dagger} b_{p^{\prime}}\right\rangle_{t}^{c} \\
& -\sum_{\sigma k} V_{\sigma \lambda_{1}}\left(\left|p^{\prime}-p\right|\right)\left\langle\psi_{\lambda_{1} l}^{\dagger} \psi_{\lambda_{2} l}\right\rangle_{t}\left\langle\psi_{\sigma k+p^{\prime}-p}^{\dagger} \psi_{\sigma k} b_{p}^{\dagger} b_{p^{\prime}}\right\rangle_{t}^{c} \\
& -\sum_{\sigma k} V_{\lambda_{2} \sigma}(|l-k|)\left\langle\psi_{\lambda_{1} l+p^{\prime}-p}^{\dagger} \psi_{\sigma l+p^{\prime}-p}\right\rangle_{t}\left\langle\psi_{\sigma k+p^{\prime}-p}^{\dagger} \psi_{\lambda_{2} k} b_{p}^{\dagger} b_{p^{\prime}}\right\rangle_{t}^{c} \\
& +\sum_{\sigma k} V_{\sigma \lambda_{1}}(|l-k|)\left\langle\psi_{\sigma l}^{\dagger} \psi_{\lambda_{2} l}\right\rangle_{t}\left\langle\psi_{\lambda_{1} k+p^{\prime}-p}^{\dagger} \psi_{\sigma k} b_{p}^{\dagger} b_{p^{\prime}}\right\rangle_{t}^{c} \\
& +\sum_{\sigma k}\left\{V_{\lambda_{2} \sigma}\left(\left|p^{\prime}\right|\right)\left\langle\psi_{\lambda_{1} l+p^{\prime}-p}^{\dagger} \psi_{\lambda_{2} l+p^{\prime}} b_{p}^{\dagger}\right\rangle_{t}-V_{\sigma \lambda_{1}}\left(\left|p^{\prime}\right|\right)\left\langle\psi_{\lambda_{1} l-p}^{\dagger} \psi_{\lambda_{2} l} b_{p}^{\dagger}\right\rangle_{t}\right\}\left\langle\psi_{\sigma k+p^{\prime}}^{\dagger} \psi_{\sigma k} b_{p^{\prime}}\right\rangle_{t} \\
& +\sum_{\sigma k}\left\{V_{\lambda_{2} \sigma}(|p|)\left\langle\psi_{\lambda_{1} l+p^{\prime}-p}^{\dagger} \psi_{\lambda_{2} l-p} b_{p^{\prime}}\right\rangle_{t}-V_{\sigma \lambda_{1}}(|p|)\left\langle\psi_{\lambda_{1} l+p^{\prime}}^{\dagger} \psi_{\lambda_{2} l} b_{p^{\prime}}\right\rangle_{t}\right\}\left\langle\psi_{\sigma k-p}^{\dagger} \psi_{\sigma k} b_{p}^{\dagger}\right\rangle_{t} \\
& -\sum_{\sigma k} V_{\lambda_{2} \sigma}(|l-k|)\left\langle\psi_{\lambda_{1} l+p^{\prime}-p}^{\dagger} \psi_{\sigma l+p^{\prime}} b_{p}^{\dagger}\right\rangle_{t}\left\langle\psi_{\sigma k+p^{\prime}}^{\dagger} \psi_{\lambda_{2} k} b_{p^{\prime}}\right\rangle_{t} \\
& +\sum_{\sigma k} V_{\sigma \lambda_{1}}\left(\left|l+p^{\prime}-k\right|\right)\left\langle\psi_{\lambda_{1} k-p}^{\dagger} \psi_{\sigma k} b_{p}^{\dagger}\right\rangle_{t}\left\langle\psi_{\sigma l+p^{\prime}}^{\dagger} \psi_{\lambda_{2} l} b_{p^{\prime}}\right\rangle_{t} \\
& -\sum_{\sigma k} V_{\lambda_{2} \sigma}(|l-k|)\left\langle\psi_{\lambda_{1} l+p^{\prime}-p}^{\dagger} \psi_{\sigma l-p} b_{p^{\prime}}\right\rangle_{t}\left\langle\psi_{\sigma k-p}^{\dagger} \psi_{\lambda_{2} k} b_{p}^{\dagger}\right\rangle_{t} \\
& +\sum_{\sigma k} V_{\sigma \lambda_{1}}(|l-p-k|)\left\langle\psi_{\lambda_{1} k+p^{\prime}}^{\dagger} \psi_{\sigma k} b_{p^{\prime}}\right\rangle_{t}\left\langle\psi_{\sigma l-p}^{\dagger} \psi_{\lambda_{2} l} b_{p}^{\dagger}\right\rangle_{t} \\
& +\sum_{\sigma k} \sum_{q} V_{\lambda_{2} \sigma}(|q|)\left\langle\psi_{\lambda_{1} l+p^{\prime}-p}^{\dagger} \psi_{\sigma k}^{\dagger} \psi_{\sigma k+q} \psi_{\lambda_{2} l-q} b_{p}^{\dagger} b_{p^{\prime}}\right\rangle_{t}^{c} \\
& -\sum_{\sigma k} \sum_{q} V_{\sigma \lambda_{1}}(|q|)\left\langle\psi_{\lambda_{1} l+p^{\prime}-p+q}^{\dagger} \psi_{\sigma k-q}^{\dagger} \psi_{\sigma k} \psi_{\lambda_{2} l} b_{p}^{\dagger} b_{p^{\prime}}\right\rangle_{t}^{c} .
\end{aligned}
$$




\section{Appendix B}

\section{The Exciton Problem for a Separable Interaction}

The eigenvalue problem which has to be solved when describing the linear response of the semiconductor in Chapter 2 is equivalent to the eigenvalue problem of an excited electron-hole pair in the presence of an attractive separable interaction. The corresponding Hamiltonian reads

$$
H=\sum_{p} H(p)=\sum_{p}\left\{H_{0}(p)+V(p)\right\} .
$$

The form of the components of the free Hamiltonian $H_{0}(p)$ is determined by the relation

$$
H_{0}(p)=\sum_{k}|k, p\rangle E(k, p)\langle k, p|
$$

where the vector $|k, p\rangle$ denotes the product state which is formed by a conduction electron with quasi impulse $k$ and a valence hole with quasi impulse $-(k+p)$. The operators which describe the electron-hole interaction are given by

$$
V(p)=-|\Phi(p)\rangle U\langle\Phi(p)|
$$

where the normalized vector which appears on the right-hand side of Equation B.3 satisfies the equation

$$
|\Phi(p)\rangle=\frac{1}{\sqrt{N}} \sum_{k}|k, p\rangle .
$$

Due to the translational symmetry of the problem the subspaces for different centerof-mass momenta decouple and the eigenvalue problem can be treated separately for each subspace. The resolvent equation for the states with a total momentum $-p$ reads

$$
\begin{aligned}
\hat{G}(p ; z) & =\hat{G}_{0}(p ; z)+\hat{G}_{0}(p ; z) V(p) \hat{G}(p ; z) \\
& =\hat{G}_{0}(p ; z)-\hat{G}_{0}(p ; z)|\Phi(p)\rangle U\langle\Phi(p)| \hat{G}(p ; z)
\end{aligned}
$$


where the resolvent operators are defined by the equations

$$
\begin{aligned}
\hat{G}_{0}(p ; z) & =\left\{z-H_{0}(p)\right\}^{-1}, \\
\hat{G}(p ; z) & =\{z-H(p)\}^{-1} .
\end{aligned}
$$

Since the potential is separable in each subspace Equation B.5 can be solved explicitly. The solution satisfies the relation

$$
\hat{G}(p ; z)=\hat{G}_{0}(p ; z)-\hat{G}_{0}(p ; z)|\Phi(p)\rangle \frac{U}{1+U\left\langle\Phi(p)\left|\hat{G}_{0}(p ; z)\right| \Phi(p)\right\rangle}\langle\Phi(p)| \hat{G}_{0}(p ; z)
$$

where the expectation value in the denominator of the second term is given by

$$
\left\langle\Phi(p)\left|\hat{G}_{0}(p ; z)\right| \Phi(p)\right\rangle=\frac{1}{N} \sum_{k} \frac{1}{z-E(k, p)} .
$$

In the thermodynamic limit the sum is replaced by an integral and one obtains

$$
\left\langle\Phi(p)\left|\hat{G}_{0}(p ; z)\right| \Phi(p)\right\rangle=\frac{1}{2 \pi} \int_{-\pi}^{\pi} d k \frac{1}{z-E_{G}(U)+T(p) \cos (k+\varphi(p))} .
$$

In Section 2.5 the resolvent has to be evaluated on the real axis with $z=\omega+i 0$. The integral from Equation B.10 can then be calculated explicitly. Depending on the position of the energy $\omega$ in the spectrum, the value of the integral is either purely real or purely imaginary:

$$
\left\langle\Phi(p)\left|\hat{G}_{0}(p ; \omega+i 0)\right| \Phi(p)\right\rangle= \begin{cases}T(p)<\omega-E_{G}(U) & : \frac{1}{\sqrt{\left(\omega-E_{G}(U)\right)^{2}-T^{2}(p)}} \\ T(p)>\left|\omega-E_{G}(U)\right| & : \frac{-i}{\sqrt{T^{2}(p)-\left(\omega-E_{G}(U)\right)^{2}}} \\ T(p)<E_{G}(U)-\omega & : \frac{-1}{\sqrt{\left(\omega-E_{G}(U)\right)^{2}-T^{2}(p)}}\end{cases}
$$

At a certain point $\omega=\omega_{e x}(p)$ below the lower band edge of the continuum states the resolvent $\hat{G}(p ; z)$ shows a resonance which indicates the existence of a bound electronhole state (exciton). As the position of this resonance is determined by the equation

$$
U\left\langle\Phi(p)\left|\hat{G}_{0}\left(p ; \omega_{e x}(p)+i 0\right)\right| \Phi(p)\right\rangle=-1
$$

the value of $\omega_{e x}(p)$ can be calculated explicitly with the help of Equation B.11 and one obtains the relation (confer Reference [49])

$$
\omega_{e x}(p)=E_{G}(U)-\sqrt{U^{2}+4\left\{t_{c}+t_{v}\right\}^{2}-16 t_{c} t_{v} \sin ^{2}\left(\frac{p}{2}\right)} .
$$


The excitonic energies for different center-of-mass momenta are arranged in a cosine shaped band. If the model parameters from Section 2.1 are used, the distance between the bottom of the exciton band at $\omega=\omega_{e x}(0)$ and the lower boundary of the continuum states at $\omega=\Delta+U$ is $\omega_{e x}(0)-\Delta-U=-0.4 \omega_{L O}$. The total width of the exciton band is then given by $\omega_{e x}(\pi)-\omega_{e x}(0)=0.5 \omega_{L O}$.

In order to calculate the susceptibility functions in Section 2.5 , it is necessary to know the coefficients of the eigenstates of the Hamiltonian $H$ which belong to the subspace of the electron-hole pairs with a vanishing center-of-mass momentum ( $p=$ $0)$. If the eigenenergy $E$ of one of these eigenstates $|\chi\rangle$ is not identical with one of the eigenvalues $E(k, 0)$ of the operator $H_{0}(0)$, the following equations

$$
E|\chi\rangle=H_{0}(0)|\chi\rangle+V(0)|\chi\rangle \quad \Leftrightarrow \quad|\chi\rangle=\hat{G}_{0}(0 ; E) V(0)|\chi\rangle
$$

and

$$
\Phi_{l}^{\chi}=\langle l, 0 \mid \chi\rangle=-\frac{U}{\sqrt{N}}\langle\Phi(0) \mid \chi\rangle \frac{1}{E-E(l, 0)}
$$

hold. The eigenenergies $E$ can then be determined by calculating the scalar product $\langle\Phi(0) \mid \chi\rangle$ with the help of Equation B.15:

$$
\begin{aligned}
\langle\Phi(0) \mid \chi\rangle & =-U \sum_{k} \frac{1}{N} \frac{1}{E-E(k, 0)}\langle\Phi(0) \mid \chi\rangle \quad \Leftrightarrow \\
-\frac{1}{U} & =\sum_{k} \frac{1}{N} \frac{1}{E-E(k, 0)} .
\end{aligned}
$$

The number of solutions $E$ for Equation B.17 is equal to the number of different eigenvalues of the operator $H_{0}(0)$. The smallest solution for the energy $E$ is identical with the energy of the exciton $E=\omega_{\text {ex }}(0)<E(0,0)$ while each of the other solutions

$$
E(k, 0)<E=\omega_{k}<E\left(k+\frac{2 \pi}{N}, 0\right) \quad(k \geq 0)
$$

lies between two neighbouring eigenvalues of $H_{0}(0)$. The coefficients for the eigenvector which describes the excitonic state, $\Phi_{l}^{e x}=\left\langle l, 0 \mid \omega_{e x}(0)\right\rangle$, and the coefficients for the eigenvectors which describe the continuum states with the energies $\omega_{k}, \Phi_{l}^{k}=\left\langle l, 0 \mid \omega_{k}\right\rangle$, are all invariant under an inversion of the system. The other eigenvectors of the operator $H(0)$ have an odd parity, their coefficients satisfy the simple equation

$$
\Phi_{l}^{k, a}=\frac{1}{\sqrt{2}}\left\{\delta_{k ; l}-\delta_{k ;-l}\right\} \quad(k \geq 0) .
$$

These antisymmetric states are not excited by the external pulse and can therefore be neglected. 


\section{Appendix C}

\section{The Four-Point Response Functions}

In this chapter of the appendix the four-point response functions, which determine the form of the four-wave-mixing signal, are calculated. In order to obtain a better overview, this chapter has been divided into two sections. In the first section the exact expressions for the different types of four-point response functions are derived. As the derivation of these functions is similar to the derivation of the two-point response functions in Section 3.4, only the most important interim results are presented here. In the second section it is shown that the exact expressions can be simplified considerably by means of approximations, if the dipole coupling function $d(x)$ and the interaction potential $v(|x|)$ meet certain requirements.

\section{C.1 Exact Expressions}

The four-point response functions which appear in Equation 3.39 can be split off into 32 different terms, if the total polarization operators $\tilde{P}$ and $\tilde{P}^{\dagger}$ are expressed with the help of the corresponding operators for the right and left movers. Fortunately, only twelve of these terms give a non-vanishing contribution since the Hamiltonian $\tilde{H}$ conserves the number of both right and left moving electrons. Because of the inversion symmetry of the system these twelve functions satisfy the following equations

$$
\begin{aligned}
& \left\langle\Omega_{0}\left|\tilde{P}_{r}\left(t_{1}\right) \tilde{P}_{r}^{\dagger}\left(t_{2}\right) \tilde{P}_{r}\left(t_{3}\right) \tilde{P}_{r}^{\dagger}\left(t_{4}\right)\right| \Omega_{0}\right\rangle=\left\langle\Omega_{0}\left|\tilde{P}_{l}\left(t_{1}\right) \tilde{P}_{l}^{\dagger}\left(t_{2}\right) \tilde{P}_{l}\left(t_{3}\right) \tilde{P}_{l}^{\dagger}\left(t_{4}\right)\right| \Omega_{0}\right\rangle, \\
& \left\langle\Omega_{0}\left|\tilde{P}_{r}\left(t_{1}\right) \tilde{P}_{r}^{\dagger}\left(t_{2}\right) \tilde{P}_{l}\left(t_{3}\right) \tilde{P}_{l}^{\dagger}\left(t_{4}\right)\right| \Omega_{0}\right\rangle=\left\langle\Omega_{0}\left|\tilde{P}_{l}\left(t_{1}\right) \tilde{P}_{l}^{\dagger}\left(t_{2}\right) \tilde{P}_{r}\left(t_{3}\right) \tilde{P}_{r}^{\dagger}\left(t_{4}\right)\right| \Omega_{0}\right\rangle, \\
& \left\langle\Omega_{0}\left|\tilde{P}_{r}\left(t_{1}\right) \tilde{P}_{l}^{\dagger}\left(t_{2}\right) \tilde{P}_{l}\left(t_{3}\right) \tilde{P}_{r}^{\dagger}\left(t_{4}\right)\right| \Omega_{0}\right\rangle=\left\langle\Omega_{0}\left|\tilde{P}_{l}\left(t_{1}\right) \tilde{P}_{r}^{\dagger}\left(t_{2}\right) \tilde{P}_{r}\left(t_{3}\right) \tilde{P}_{l}^{\dagger}\left(t_{4}\right)\right| \Omega_{0}\right\rangle
\end{aligned}
$$

and

$$
\begin{aligned}
& \left\langle\Omega_{0}\left|\tilde{P}_{r}\left(t_{1}\right) \tilde{P}_{r}\left(t_{2}\right) \tilde{P}_{r}^{\dagger}\left(t_{3}\right) \tilde{P}_{r}^{\dagger}\left(t_{4}\right)\right| \Omega_{0}\right\rangle=\left\langle\Omega_{0}\left|\tilde{P}_{l}\left(t_{1}\right) \tilde{P}_{l}\left(t_{2}\right) \tilde{P}_{l}^{\dagger}\left(t_{3}\right) \tilde{P}_{l}^{\dagger}\left(t_{4}\right)\right| \Omega_{0}\right\rangle, \\
& \left\langle\Omega_{0}\left|\tilde{P}_{r}\left(t_{1}\right) \tilde{P}_{l}\left(t_{2}\right) \tilde{P}_{r}^{\dagger}\left(t_{3}\right) \tilde{P}_{l}^{\dagger}\left(t_{4}\right)\right| \Omega_{0}\right\rangle=\left\langle\Omega_{0}\left|\tilde{P}_{l}\left(t_{1}\right) \tilde{P}_{r}\left(t_{2}\right) \tilde{P}_{l}^{\dagger}\left(t_{3}\right) \tilde{P}_{r}^{\dagger}\left(t_{4}\right)\right| \Omega_{0}\right\rangle, \\
& \left\langle\Omega_{0}\left|\tilde{P}_{r}\left(t_{1}\right) \tilde{P}_{l}\left(t_{2}\right) \tilde{P}_{l}^{\dagger}\left(t_{3}\right) \tilde{P}_{r}^{\dagger}\left(t_{4}\right)\right| \Omega_{0}\right\rangle=\left\langle\Omega_{0}\left|\tilde{P}_{l}\left(t_{1}\right) \tilde{P}_{r}\left(t_{2}\right) \tilde{P}_{r}^{\dagger}\left(t_{3}\right) \tilde{P}_{l}^{\dagger}\left(t_{4}\right)\right| \Omega_{0}\right\rangle .
\end{aligned}
$$


Therefore, it is sufficient to calculate the response functions on the left-hand sides of Equations C.1 to C.6.

In the following the response functions which appear in Equations C. 1 to C.3 are referred to as excitonic four-point response functions since they describe transitions between the subspaces with no and one valence hole. Correspondingly, the functions in Equations C.4 to C.6 are called biexcitonic four-point response functions because they describe processes in which two electron-hole pairs are created and annihilated. In order to calculate the four-point response functions, it is necessary to know the expectation values of the corresponding products of field operators which can be evaluated exactly with the help of the auxiliary functions introduced in Appendix D.

The excitonic response function on the left-hand side of Equation C.1 describes processes in which only pairs of valence holes and right moving electrons are created and annihilated. The spatial correlation function which is related to this response function satisfies the following equation

$$
\begin{aligned}
& \left\langle\tilde{\psi}_{e r}\left(x_{1}, t_{1}\right) \tilde{\psi}_{h}\left(y_{1}, t_{1}\right) \tilde{\psi}_{h}^{\dagger}\left(y_{2}, t_{2}\right) \tilde{\psi}_{e r}^{\dagger}\left(x_{2}, t_{2}\right) \tilde{\psi}_{e r}\left(x_{3}, t_{3}\right) \tilde{\psi}_{h}\left(y_{3}, t_{3}\right) \tilde{\psi}_{h}^{\dagger}\left(y_{4}, t_{4}\right) \tilde{\psi}_{e r}^{\dagger}\left(x_{4}, t_{4}\right)\right\rangle_{0} \\
& =e^{s_{X ; r}\left(t_{1}, t_{2}, x_{1}-y_{1}\right)} e^{s_{X ; r}^{*}\left(t_{2}, t_{1}, x_{2}-y_{1}\right)} e^{s_{X ; r}\left(t_{3}, t_{4}, x_{3}-y_{4}\right)} e^{s_{X ; r}^{*}\left(t_{4}, t_{3}, x_{4}-y_{4}\right)} \\
& \times e^{S_{X ; r r}\left(t_{1}, t_{3}, t_{2}, t_{4} ; y_{1}-y_{4} ; x_{1}-y_{1}, x_{3}-y_{4}, y_{1}-y_{4}\right)} e^{S_{X ; r r}^{*}\left(t_{4}, t_{2}, t_{3}, t_{1} ; y_{1}-y_{4} ; x_{4}-y_{4}, x_{2}-y_{1},-y_{1}+y_{4}\right)} \\
& \times e^{i\left(t_{1}-t_{2}+t_{3}-t_{4}\right) \varepsilon(0)}\left\langle\psi_{h ; 0}\left(y_{1}, t_{1}\right) \psi_{h ; 0}^{\dagger}\left(y_{2}, t_{2}\right)\right\rangle_{0}\left\langle\psi_{h ; 0}\left(y_{3}, t_{3}\right) \psi_{h ; 0}^{\dagger}\left(y_{4}, t_{4}\right)\right\rangle_{0} \\
& \times\left\{\left\langle\psi_{e r ; 0}\left(x_{1}, t_{1}\right) \psi_{e r ; 0}^{\dagger}\left(x_{4}, t_{4}\right)\right\rangle_{0}\left\langle\psi_{e r ; 0}^{\dagger}\left(x_{2}, t_{2}\right) \psi_{e r ; 0}\left(x_{3}, t_{3}\right)\right\rangle_{0}\right. \\
& \left.\quad+\left\langle\psi_{e r ; 0}\left(x_{1}, t_{1}\right) \psi_{e r ; 0}^{\dagger}\left(x_{2}, t_{2}\right)\right\rangle_{0}\left\langle\psi_{e r ; 0}\left(x_{3}, t_{3}\right) \psi_{e r ; 0}^{\dagger}\left(x_{4}, t_{4}\right)\right\rangle_{0}\right\} .
\end{aligned}
$$

The auxiliary function $S_{X ; r r}$ has been introduced in order to obtain a compact expression. It is defined by the relation

$$
\begin{aligned}
S_{X ; r r}\left(t_{1}, t_{2}, t_{3}, t_{4} ; y^{\prime} ; x_{1}, x_{2}, y\right) & =H^{*}\left(v_{f}\left(t_{1}-t_{4}\right)-y^{\prime}, v_{f}\left(t_{1}-t_{2}\right)-y^{\prime}\right) \\
& +H^{*}\left(v_{f}\left(t_{3}-t_{2}\right)+y^{\prime}, v_{f}\left(t_{1}-t_{2}\right)+y^{\prime}\right) \\
& +G^{*}\left(v_{f}\left(t_{1}-t_{4}\right)-y-x_{1}, v_{f}\left(t_{1}-t_{2}\right)-y-x_{1}\right) \\
& +G^{*}\left(v_{f}\left(t_{3}-t_{2}\right)-y+x_{2}, v_{f}\left(t_{1}-t_{2}\right)-y+x_{2}\right) .
\end{aligned}
$$

The excitonic response functions on the left-hand sides of Equations C.2 and C.3 represent processes which involve the creation and annihilation of both left and right moving electrons. The corresponding spatial correlation functions are given by

$$
\begin{aligned}
& \left\langle\tilde{\psi}_{e r}\left(x_{1}, t_{1}\right) \tilde{\psi}_{h}\left(y_{1}, t_{1}\right) \tilde{\psi}_{h}^{\dagger}\left(y_{2}, t_{2}\right) \tilde{\psi}_{e r}^{\dagger}\left(x_{2}, t_{2}\right) \tilde{\psi}_{e l}\left(x_{3}, t_{3}\right) \tilde{\psi}_{h}\left(y_{3}, t_{3}\right) \tilde{\psi}_{h}^{\dagger}\left(y_{4}, t_{4}\right) \tilde{\psi}_{e l}^{\dagger}\left(x_{4}, t_{4}\right)\right\rangle_{0} \\
& =e^{s_{X ; r}\left(t_{1}, t_{2}, x_{1}-y_{1}\right)} e^{s_{X ; r}^{*}\left(t_{2}, t_{1}, x_{2}-y_{1}\right)} e^{s_{X ; l}\left(t_{3}, t_{4}, x_{3}-y_{4}\right)} e^{s_{X ; l}^{*}\left(t_{4}, t_{3}, x_{4}-y_{4}\right)} \\
& \times e^{S_{X ; r l}\left(t_{1}, t_{3}, t_{2}, t_{4} ; y_{1}-y_{4} ; x_{1}-y_{1}, x_{3}-y_{4}, y_{1}-y_{4}\right)} e^{S_{X ; l r}^{*}\left(t_{4}, t_{2}, t_{3}, t_{1} ; y_{1}-y_{4} ; x_{4}-y_{4}, x_{2}-y_{1},-y_{1}+y_{4}\right)} \\
& \times e^{i\left(t_{1}-t_{2}+t_{3}-t_{4}\right) \varepsilon(0)}\left\langle\psi_{h ; 0}\left(y_{1}, t_{1}\right) \psi_{h ; 0}^{\dagger}\left(y_{2}, t_{2}\right)\right\rangle_{0}\left\langle\psi_{h ; 0}\left(y_{3}, t_{3}\right) \psi_{h ; 0}^{\dagger}\left(y_{4}, t_{4}\right)\right\rangle_{0} \\
& \times\left\langle\psi_{e r ; 0}\left(x_{1}, t_{1}\right) \psi_{e r ; 0}^{\dagger}\left(x_{2}, t_{2}\right)\right\rangle_{0}\left\langle\psi_{e l ; 0}\left(x_{3}, t_{3}\right) \psi_{e l ; 0}^{\dagger}\left(x_{4}, t_{4}\right)\right\rangle_{0}
\end{aligned}
$$


and

$$
\begin{aligned}
& \left\langle\tilde{\psi}_{e r}\left(x_{1}, t_{1}\right) \tilde{\psi}_{h}\left(y_{1}, t_{1}\right) \tilde{\psi}_{h}^{\dagger}\left(y_{2}, t_{2}\right) \tilde{\psi}_{e l}^{\dagger}\left(x_{2}, t_{2}\right) \tilde{\psi}_{e l}\left(x_{3}, t_{3}\right) \tilde{\psi}_{h}\left(y_{3}, t_{3}\right) \tilde{\psi}_{h}^{\dagger}\left(y_{4}, t_{4}\right) \tilde{\psi}_{e r}^{\dagger}\left(x_{4}, t_{4}\right)\right\rangle_{0} \\
& =e^{s_{X ; r}\left(t_{1}, t_{2}, x_{1}-y_{1}\right)} e^{s_{X ; l}^{*}\left(t_{2}, t_{1}, x_{2}-y_{1}\right)} e^{s_{X ; l}\left(t_{3}, t_{4}, x_{3}-y_{4}\right)} e^{s_{X ; r}^{*}\left(t_{4}, t_{3}, x_{4}-y_{4}\right)} \\
& \times e^{S_{X ; r l}\left(t_{1}, t_{3}, t_{2}, t_{4} ; y_{1}-y_{4} ; x_{1}-y_{1}, x_{3}-y_{4}, y_{1}-y_{4}\right)} e^{S_{X ; r l}^{*}\left(t_{4}, t_{2}, t_{3}, t_{1} ; y_{1}-y_{4} ; x_{4}-y_{4}, x_{2}-y_{1},-y_{1}+y_{4}\right)} \\
& \times e^{i\left(t_{1}-t_{2}+t_{3}-t_{4}\right) \varepsilon(0)}\left\langle\psi_{h ; 0}\left(y_{1}, t_{1}\right) \psi_{h ; 0}^{\dagger}\left(y_{2}, t_{2}\right)\right\rangle_{0}\left\langle\psi_{h ; 0}\left(y_{3}, t_{3}\right) \psi_{h ; 0}^{\dagger}\left(y_{4}, t_{4}\right)\right\rangle_{0} \\
& \times\left\langle\psi_{e r ; 0}\left(x_{1}, t_{1}\right) \psi_{e r ; 0}^{\dagger}\left(x_{4}, t_{4}\right)\right\rangle_{0}\left\langle\psi_{e l ; 0}^{\dagger}\left(x_{2}, t_{2}\right) \psi_{e l ; 0}\left(x_{3}, t_{3}\right)\right\rangle_{0} .
\end{aligned}
$$

The new auxiliary functions which have been introduced in Equations C.9 and C.10 are defined by the two relations

$$
\begin{aligned}
S_{X ; r l}\left(t_{1}, t_{2}, t_{3}, t_{4} ; y^{\prime} ; x_{1}, x_{2}, y\right) & =H^{*}\left(v_{f}\left(t_{1}-t_{4}\right)-y^{\prime}, v_{f}\left(t_{1}-t_{2}\right)-y^{\prime}\right) \\
& +H^{*}\left(v_{f}\left(t_{3}-t_{2}\right)+y^{\prime}, v_{f}\left(t_{1}-t_{2}\right)+y^{\prime}\right) \\
& +G^{*}\left(v_{f}\left(t_{1}-t_{4}\right)-y-x_{1}, v_{f}\left(t_{1}-t_{2}\right)-y-x_{1}\right) \\
& +G^{*}\left(v_{f}\left(t_{3}-t_{2}\right)+y-x_{2}, v_{f}\left(t_{1}-t_{2}\right)+y-x_{2}\right)
\end{aligned}
$$

and

$$
\begin{aligned}
S_{X ; l r}\left(t_{1}, t_{2}, t_{3}, t_{4} ; y^{\prime} ; x_{1}, x_{2}, y\right) & =H^{*}\left(v_{f}\left(t_{1}-t_{4}\right)-y^{\prime}, v_{f}\left(t_{1}-t_{2}\right)-y^{\prime}\right) \\
& +H^{*}\left(v_{f}\left(t_{3}-t_{2}\right)+y^{\prime}, v_{f}\left(t_{1}-t_{2}\right)+y^{\prime}\right) \\
& +G^{*}\left(v_{f}\left(t_{1}-t_{4}\right)+y+x_{1}, v_{f}\left(t_{1}-t_{2}\right)+y+x_{1}\right) \\
& +G^{*}\left(v_{f}\left(t_{3}-t_{2}\right)-y+x_{2}, v_{f}\left(t_{1}-t_{2}\right)-y+x_{2}\right) .
\end{aligned}
$$

Due to the structure of the excitonic response functions there exists only one way to combine the hole operators. Therefore all spatial correlation functions share the common factor

$$
\left\langle\psi_{h ; 0}\left(y_{1}, t_{1}\right) \psi_{h ; 0}^{\dagger}\left(y_{2}, t_{2}\right)\right\rangle_{0}\left\langle\psi_{h ; 0}\left(y_{3}, t_{3}\right) \psi_{h ; 0}^{\dagger}\left(y_{4}, t_{4}\right)\right\rangle_{0} .
$$

If all four electronic field operators belong to the same sort of electrons, two different pairings of the operators $\psi_{e \sigma}$ and $\psi_{e \sigma}^{\dagger}$ are possible, as can be seen in Equation C.7. On the other hand, the expressions for the correlation functions in Equations C.9 and C.10 contain only one combination of electronic pair functions since field operators of both sorts of electrons appear in the corresponding operator products. 
The biexcitonic response function on the left-hand side of Equation C. 4 can be calculated with the help of the spatial correlation function

$$
\begin{aligned}
& \left\langle\tilde{\psi}_{e r}\left(x_{1}, t_{1}\right) \tilde{\psi}_{h}\left(y_{1}, t_{1}\right) \tilde{\psi}_{e r}\left(x_{2}, t_{2}\right) \tilde{\psi}_{h}\left(y_{2}, t_{2}\right) \tilde{\psi}_{h}^{\dagger}\left(y_{3}, t_{3}\right) \tilde{\psi}_{e r}^{\dagger}\left(x_{3}, t_{3}\right) \tilde{\psi}_{h}^{\dagger}\left(y_{4}, t_{4}\right) \tilde{\psi}_{e r}^{\dagger}\left(x_{4}, t_{4}\right)\right\rangle_{0} \\
& =e^{i\left(t_{2}-t_{3}\right) 2 \varepsilon\left(y_{1}-y_{2}\right)} e^{s_{X ; r}\left(t_{1}, t_{4}, x_{1}-y_{1}\right)} e^{s_{X ; r}\left(t_{2}, t_{3}, x_{2}-y_{2}\right)} e^{s_{X ; r}^{*}\left(t_{3}, t_{2}, x_{3}-y_{2}\right)} e^{s_{X ; r}^{*}\left(t_{4}, t_{1}, x_{4}-y_{1}\right)} \\
& \times e^{S_{X X ; r r}\left(t_{1}, t_{2}, t_{4}, t_{3} ; y_{1}-y_{2} ; x_{1}-y_{1}, x_{2}-y_{2}, y_{1}-y_{2}\right)} e^{S_{X X ; r r}^{*}\left(t_{4}, t_{3}, t_{1}, t_{2} ; y_{1}-y_{2} ; x_{4}-y_{1}, x_{3}-y_{2}, y_{1}-y_{2}\right)} \\
& \times e^{i\left(t_{1}+t_{2}-t_{3}-t_{4}\right) \varepsilon(0)}\left\langle\psi_{h ; 0}\left(y_{1}, t_{1}\right) \psi_{h ; 0}^{\dagger}\left(y_{4}, t_{4}\right)\right\rangle_{0}\left\langle\psi_{h ; 0}\left(y_{2}, t_{2}\right) \psi_{h ; 0}^{\dagger}\left(y_{3}, t_{3}\right)\right\rangle_{0} \\
& \times\left\{\left\langle\psi_{e r ; 0}\left(x_{1}, t_{1}\right) \psi_{e r ; 0}^{\dagger}\left(x_{4}, t_{4}\right)\right\rangle_{0}\left\langle\psi_{e r ; 0}\left(x_{2}, t_{2}\right) \psi_{e r ; 0}^{\dagger}\left(x_{3}, t_{3}\right)\right\rangle_{0}\right. \\
& \left.-\left\langle\psi_{e r ; 0}\left(x_{1}, t_{1}\right) \psi_{e r ; 0}^{\dagger}\left(x_{3}, t_{3}\right)\right\rangle_{0}\left\langle\psi_{e r ; 0}\left(x_{2}, t_{2}\right) \psi_{e r ; 0}^{\dagger}\left(x_{4}, t_{4}\right)\right\rangle_{0}\right\} \\
& +e^{i\left(t_{2}-t_{3}\right) 2 \varepsilon\left(y_{1}-y_{2}\right)} e^{s_{X ; r}\left(t_{1}, t_{3}, x_{1}-y_{1}\right)} e^{s_{X ; r}\left(t_{2}, t_{4}, x_{2}-y_{2}\right)} e^{s_{X ; r}^{*}\left(t_{3}, t_{1}, x_{3}-y_{1}\right)} e^{s_{X ; r}^{*}\left(t_{4}, t_{2}, x_{4}-y_{2}\right)} \\
& \times e^{S_{X X ; r r}\left(t_{1}, t_{2}, t_{3}, t_{4} ; y_{1}-y_{2} ; x_{1}-y_{1}, x_{2}-y_{2}, y_{1}-y_{2}\right)} e^{S_{X X ; r r}^{*}\left(t_{4}, t_{3}, t_{2}, t_{1} ; y_{1}-y_{2} ; x_{4}-y_{2}, x_{3}-y_{1},-y_{1}+y_{2}\right)} \\
& \times e^{i\left(t_{1}+t_{2}-t_{3}-t_{4}\right) \varepsilon(0)}\left\langle\psi_{h ; 0}\left(y_{1}, t_{1}\right) \psi_{h ; 0}^{\dagger}\left(y_{3}, t_{3}\right)\right\rangle_{0}\left\langle\psi_{h ; 0}\left(y_{2}, t_{2}\right) \psi_{h ; 0}^{\dagger}\left(y_{4}, t_{4}\right)\right\rangle_{0} \\
& \times\left\{\left\langle\psi_{e r ; 0}\left(x_{1}, t_{1}\right) \psi_{e r ; 0}^{\dagger}\left(x_{3}, t_{3}\right)\right\rangle_{0}\left\langle\psi_{e r ; 0}\left(x_{2}, t_{2}\right) \psi_{e r ; 0}^{\dagger}\left(x_{4}, t_{4}\right)\right\rangle_{0}\right. \\
& \left.-\left\langle\psi_{e r ; 0}\left(x_{1}, t_{1}\right) \psi_{e r ; 0}^{\dagger}\left(x_{4}, t_{4}\right)\right\rangle_{0}\left\langle\psi_{e r ; 0}\left(x_{2}, t_{2}\right) \psi_{e r ; 0}^{\dagger}\left(x_{3}, t_{3}\right)\right\rangle_{0}\right\}
\end{aligned}
$$

where the auxiliary function $S_{X X ; r r}$ is given by

$$
\begin{aligned}
S_{X X ; r r}\left(t_{1}, t_{2}, t_{3}, t_{4} ; y^{\prime} ; x_{1}, x_{2}, y\right) & =H^{*}\left(v_{f}\left(t_{1}-t_{4}\right)-y^{\prime}, v_{f}\left(t_{1}-t_{2}\right)-y^{\prime}\right) \\
& +H^{*}\left(v_{f}\left(t_{2}-t_{3}\right)-y^{\prime}, v_{f}\left(t_{1}-t_{2}\right)+y^{\prime}\right) \\
& +G^{*}\left(v_{f}\left(t_{1}-t_{4}\right)-y-x_{1}, v_{f}\left(t_{1}-t_{2}\right)-y-x_{1}\right) \\
& +G^{*}\left(v_{f}\left(t_{2}-t_{3}\right)+y-x_{2}, y-x_{2}\right) \\
& +G^{*}\left(-y+x_{2}, v_{f}\left(t_{1}-t_{2}\right)-y+x_{2}\right) .
\end{aligned}
$$

Unlike the case of the excitonic correlation function in Equation C.7, there are two different ways to contract the four hole operators of the biexcitonic correlation function in Equation C.13. Therefore the different terms on the right-hand side of Equation C.13 contain either the factor

$$
\left\langle\psi_{h ; 0}\left(y_{1}, t_{1}\right) \psi_{h ; 0}^{\dagger}\left(y_{4}, t_{4}\right)\right\rangle_{0}\left\langle\psi_{h ; 0}\left(y_{2}, t_{2}\right) \psi_{h ; 0}^{\dagger}\left(y_{3}, t_{3}\right)\right\rangle_{0}
$$

or the factor

$$
\left\langle\psi_{h ; 0}\left(y_{1}, t_{1}\right) \psi_{h ; 0}^{\dagger}\left(y_{3}, t_{3}\right)\right\rangle_{0}\left\langle\psi_{h ; 0}\left(y_{2}, t_{2}\right) \psi_{h ; 0}^{\dagger}\left(y_{4}, t_{4}\right)\right\rangle_{0}
$$

The same is true for the biexcitonic response functions which describe mixed excitation processes. For example, the spatial correlation functions for the response func- 
tions on the left-hand sides of Equations C.5 and C.6 satisfy the following equations

$$
\begin{aligned}
& \left\langle\tilde{\psi}_{e r}\left(x_{1}, t_{1}\right) \tilde{\psi}_{h}\left(y_{1}, t_{1}\right) \tilde{\psi}_{e l}\left(x_{2}, t_{2}\right) \tilde{\psi}_{h}\left(y_{2}, t_{2}\right) \tilde{\psi}_{h}^{\dagger}\left(y_{3}, t_{3}\right) \tilde{\psi}_{e r}^{\dagger}\left(x_{3}, t_{3}\right) \tilde{\psi}_{h}^{\dagger}\left(y_{4}, t_{4}\right) \tilde{\psi}_{e l}^{\dagger}\left(x_{4}, t_{4}\right)\right\rangle_{0} \\
& =e^{i\left(t_{2}-t_{3}\right) 2 \varepsilon\left(y_{1}-y_{2}\right)} e^{s_{X ; r}\left(t_{1}, t_{3}, x_{1}-y_{1}\right)} e^{s_{X ; l}\left(t_{2}, t_{4}, x_{2}-y_{2}\right)} e^{s_{X ; r}^{*}\left(t_{3}, t_{1}, x_{3}-y_{1}\right)} e^{s_{X ; l}^{*}\left(t_{4}, t_{2}, x_{4}-y_{2}\right)} \\
& \times e^{S_{X X ; r l}\left(t_{1}, t_{2}, t_{3}, t_{4} ; y_{1}-y_{2} ; x_{1}-y_{1}, x_{2}-y_{2}, y_{1}-y_{2}\right)} e^{S_{X X ; l r}^{*}\left(t_{4}, t_{3}, t_{2}, t_{1} ; y_{1}-y_{2} ; x_{4}-y_{2}, x_{3}-y_{1},-y_{1}+y_{2}\right)} \\
& \times e^{i\left(t_{1}+t_{2}-t_{3}-t_{4}\right) \varepsilon(0)}\left\langle\psi_{h ; 0}\left(y_{1}, t_{1}\right) \psi_{h ; 0}^{\dagger}\left(y_{3}, t_{3}\right)\right\rangle_{0}\left\langle\psi_{h ; 0}\left(y_{2}, t_{2}\right) \psi_{h ; 0}^{\dagger}\left(y_{4}, t_{4}\right)\right\rangle_{0} \\
& \times\left\langle\psi_{e r ; 0}\left(x_{1}, t_{1}\right) \psi_{e r ; 0}^{\dagger}\left(x_{3}, t_{3}\right)\right\rangle_{0}\left\langle\psi_{e l ; 0}\left(x_{2}, t_{2}\right) \psi_{e l ; 0}^{\dagger}\left(x_{4}, t_{4}\right)\right\rangle_{0} \\
& -e^{i\left(t_{2}-t_{3}\right) 2 \varepsilon\left(y_{1}-y_{2}\right)} e^{s_{X ; r}\left(t_{1}, t_{4}, x_{1}-y_{1}\right)} e^{s_{X ; l}\left(t_{2}, t_{3}, x_{2}-y_{2}\right)} e^{s_{X ; r}^{*}\left(t_{3}, t_{2}, x_{3}-y_{2}\right)} e^{s_{X ; l}^{*}\left(t_{4}, t_{1}, x_{4}-y_{1}\right)} \\
& \times e^{S_{X X ; r l}\left(t_{1}, t_{2}, t_{4}, t_{3} ; y_{1}-y_{2} ; x_{1}-y_{1}, x_{2}-y_{2}, y_{1}-y_{2}\right)} e^{S_{X X ; l r}^{*}\left(t_{4}, t_{3}, t_{1}, t_{2} ; y_{1}-y_{2} ; x_{4}-y_{1}, x_{3}-y_{2}, y_{1}-y_{2}\right)} \\
& \times e^{i\left(t_{1}+t_{2}-t_{3}-t_{4}\right) \varepsilon(0)}\left\langle\psi_{h ; 0}\left(y_{1}, t_{1}\right) \psi_{h ; 0}^{\dagger}\left(y_{4}, t_{4}\right)\right\rangle_{0}\left\langle\psi_{h ; 0}\left(y_{2}, t_{2}\right) \psi_{h ; 0}^{\dagger}\left(y_{3}, t_{3}\right)\right\rangle_{0} \\
& \times\left\langle\psi_{e r ; 0}\left(x_{1}, t_{1}\right) \psi_{e r ; 0}^{\dagger}\left(x_{3}, t_{3}\right)\right\rangle_{0}\left\langle\psi_{e l ; 0}\left(x_{2}, t_{2}\right) \psi_{e l ; 0}^{\dagger}\left(x_{4}, t_{4}\right)\right\rangle_{0}
\end{aligned}
$$

and

$$
\begin{aligned}
& \left\langle\tilde{\psi}_{e r}\left(x_{1}, t_{1}\right) \tilde{\psi}_{h}\left(y_{1}, t_{1}\right) \tilde{\psi}_{e l}\left(x_{2}, t_{2}\right) \tilde{\psi}_{h}\left(y_{2}, t_{2}\right) \tilde{\psi}_{h}^{\dagger}\left(y_{3}, t_{3}\right) \tilde{\psi}_{e l}^{\dagger}\left(x_{3}, t_{3}\right) \tilde{\psi}_{h}^{\dagger}\left(y_{4}, t_{4}\right) \tilde{\psi}_{e r}^{\dagger}\left(x_{4}, t_{4}\right)\right\rangle_{0} \\
& =e^{i\left(t_{2}-t_{3}\right) 2 \varepsilon\left(y_{1}-y_{2}\right)} e^{s_{X ; r}\left(t_{1}, t_{4}, x_{1}-y_{1}\right)} e^{s_{X ; l}\left(t_{2}, t_{3}, x_{2}-y_{2}\right)} e^{s_{X ; l}^{*}\left(t_{3}, t_{2}, x_{3}-y_{2}\right)} e^{s_{X ; r}^{*}\left(t_{4}, t_{1}, x_{4}-y_{1}\right)} \\
& \times e^{S_{X X ; r l}\left(t_{1}, t_{2}, t_{4}, t_{3} ; y_{1}-y_{2} ; x_{1}-y_{1}, x_{2}-y_{2}, y_{1}-y_{2}\right)} e^{S_{X X ; r l}^{*}\left(t_{4}, t_{3}, t_{1}, t_{2} ; y_{1}-y_{2} ; x_{4}-y_{1}, x_{3}-y_{2}, y_{1}-y_{2}\right)} \\
& \times e^{i\left(t_{1}+t_{2}-t_{3}-t_{4}\right) \varepsilon(0)}\left\langle\psi_{h ; 0}\left(y_{1}, t_{1}\right) \psi_{h ; 0}^{\dagger}\left(y_{4}, t_{4}\right)\right\rangle_{0}\left\langle\psi_{h ; 0}\left(y_{2}, t_{2}\right) \psi_{h ; 0}^{\dagger}\left(y_{3}, t_{3}\right)\right\rangle_{0} \\
& \times\left\langle\psi_{e r ; 0}\left(x_{1}, t_{1}\right) \psi_{e r ; 0}^{\dagger}\left(x_{4}, t_{4}\right)\right\rangle_{0}\left\langle\psi_{e l ; 0}\left(x_{2}, t_{2}\right) \psi_{e l ; 0}^{\dagger}\left(x_{3}, t_{3}\right)\right\rangle_{0} \\
& -e^{i\left(t_{2}-t_{3}\right) 2 \varepsilon\left(y_{1}-y_{2}\right)} e^{s_{X ; r}\left(t_{1}, t_{3}, x_{1}-y_{1}\right)} e^{s_{X ; l}\left(t_{2}, t_{4}, x_{2}-y_{2}\right)} e^{s_{X ; l}^{*}\left(t_{3}, t_{1}, x_{3}-y_{1}\right)} e^{s_{X ; r}^{*}\left(t_{4}, t_{2}, x_{4}-y_{2}\right)} \\
& \times e^{S_{X X ; r l}\left(t_{1}, t_{2}, t_{3}, t_{4} ; y_{1}-y_{2} ; x_{1}-y_{1}, x_{2}-y_{2}, y_{1}-y_{2}\right)} e^{S_{X X ; r l}^{*}\left(t_{4}, t_{3}, t_{2}, t_{1} ; y_{1}-y_{2} ; x_{4}-y_{2}, x_{3}-y_{1},-y_{1}+y_{2}\right)} \\
& \times e^{i\left(t_{1}+t_{2}-t_{3}-t_{4}\right) \varepsilon(0)}\left\langle\psi_{h ; 0}\left(y_{1}, t_{1}\right) \psi_{h ; 0}^{\dagger}\left(y_{3}, t_{3}\right)\right\rangle_{0}\left\langle\psi_{h ; 0}\left(y_{2}, t_{2}\right) \psi_{h ; 0}^{\dagger}\left(y_{4}, t_{4}\right)\right\rangle_{0} \\
& \times\left\langle\psi_{e r ; 0}\left(x_{1}, t_{1}\right) \psi_{e r ; 0}^{\dagger}\left(x_{4}, t_{4}\right)\right\rangle_{0}\left\langle\psi_{e l ; 0}\left(x_{2}, t_{2}\right) \psi_{e l ; 0}^{\dagger}\left(x_{3}, t_{3}\right)\right\rangle_{0} .
\end{aligned}
$$

Here, the auxiliary functions are defined by the two relations

$$
\begin{aligned}
S_{X X ; r l}\left(t_{1}, t_{2}, t_{3}, t_{4} ; y^{\prime} ; x_{1}, x_{2}, y\right) & =H^{*}\left(v_{f}\left(t_{1}-t_{4}\right)-y^{\prime}, v_{f}\left(t_{1}-t_{2}\right)-y^{\prime}\right) \\
& +H^{*}\left(v_{f}\left(t_{2}-t_{3}\right)-y^{\prime}, v_{f}\left(t_{1}-t_{2}\right)+y^{\prime}\right) \\
& +G^{*}\left(v_{f}\left(t_{1}-t_{4}\right)-y-x_{1}, v_{f}\left(t_{1}-t_{2}\right)-y-x_{1}\right) \\
& +G^{*}\left(v_{f}\left(t_{2}-t_{3}\right)-y+x_{2},-y+x_{2}\right) \\
& +G^{*}\left(y-x_{2}, v_{f}\left(t_{1}-t_{2}\right)+y-x_{2}\right)
\end{aligned}
$$

and

$$
\begin{aligned}
S_{X X ; l r}\left(t_{1}, t_{2}, t_{3}, t_{4} ; y^{\prime} ; x_{1}, x_{2}, y\right) & =H^{*}\left(v_{f}\left(t_{1}-t_{4}\right)-y^{\prime}, v_{f}\left(t_{1}-t_{2}\right)-y^{\prime}\right) \\
& +H^{*}\left(v_{f}\left(t_{2}-t_{3}\right)-y^{\prime}, v_{f}\left(t_{1}-t_{2}\right)+y^{\prime}\right) \\
& +G^{*}\left(v_{f}\left(t_{1}-t_{4}\right)+y+x_{1}, v_{f}\left(t_{1}-t_{2}\right)+y+x_{1}\right) \\
& +G^{*}\left(v_{f}\left(t_{2}-t_{3}\right)+y-x_{2}, y-x_{2}\right) \\
& +G^{*}\left(-y+x_{2}, v_{f}\left(t_{1}-t_{2}\right)-y+x_{2}\right) .
\end{aligned}
$$


As the number of the possible contractions of the electronic field operators in biexcitonic correlation functions is equal to the number of the possible contractions in the corresponding excitonic correlation functions the former always consist of twice as many terms as the latter.

The four-point response functions can be calculated by integrating the corresponding spatial correlation functions with respect to all position variables. The connected parts are then obtained by applying the definitions 3.40 and 3.41. In the case of the excitonic response function which is related to the spatial correlation function in Equation C.7 the procedure yields the following formula

$$
\begin{aligned}
& \lim _{L \rightarrow \infty} \frac{1}{L}\left\langle\Omega_{0}\left|\tilde{P}_{r}\left(t_{1}\right) \tilde{P}_{r}^{\dagger}\left(t_{2}\right) \tilde{P}_{r}\left(t_{3}\right) \tilde{P}_{r}^{\dagger}\left(t_{4}\right)\right| \Omega_{0}\right\rangle_{c}=\left\langle\left\langle\tilde{P}_{r}\left(t_{1}\right) \tilde{P}_{r}^{\dagger}\left(t_{2}\right) \tilde{P}_{r}\left(t_{3}\right) \tilde{P}_{r}^{\dagger}\left(t_{4}\right)\right\rangle\right\rangle_{c} \\
& =\left\langle\left\langle\tilde{P}_{r}\left(t_{1}\right) \tilde{P}_{r}^{\dagger}\left(t_{2}\right) \tilde{P}_{r}\left(t_{3}\right) \tilde{P}_{r}^{\dagger}\left(t_{4}\right)\right\rangle\right\rangle_{c}^{I}+\left\langle\left\langle\tilde{P}_{r}\left(t_{1}\right) \tilde{P}_{r}^{\dagger}\left(t_{2}\right) \tilde{P}_{r}\left(t_{3}\right) \tilde{P}_{r}^{\dagger}\left(t_{4}\right)\right\rangle\right\rangle_{c}^{I I}
\end{aligned}
$$

in the thermodynamic limit where the two contributions on the right-hand side of Equation C.19 are defined by the relations

$$
\begin{aligned}
& \left\langle\left\langle\tilde{P}_{r}\left(t_{1}\right) \tilde{P}_{r}^{\dagger}\left(t_{2}\right) \tilde{P}_{r}\left(t_{3}\right) \tilde{P}_{r}^{\dagger}\left(t_{4}\right)\right\rangle\right\rangle_{c}^{I}=e^{-i\left(t_{1}-t_{2}+t_{3}-t_{4}\right)\left(E_{h}-\varepsilon(0)\right)} \\
& \times \int_{0}^{\infty} \frac{d k}{2 \pi} e^{i k v_{f}\left(t_{2}-t_{1}\right)} \int_{-\infty}^{\infty} d \tilde{x}_{1} e^{i k \tilde{x}_{1}} d\left(\tilde{x}_{1}\right) e^{s_{X ; r}\left(t_{1}, t_{2}, \tilde{x}_{1}\right)} \int_{-\infty}^{\infty} d \tilde{x}_{2} e^{-i k \tilde{x}_{2}} d\left(\tilde{x}_{2}\right) e^{s_{X ; r}^{*}\left(t_{2}, t_{1}, \tilde{x}_{2}\right)} \\
& \times \int_{0}^{\infty} \frac{d \bar{k}}{2 \pi} e^{i \bar{k} v_{f}\left(t_{4}-t_{3}\right)} \int_{-\infty}^{\infty} d \tilde{x}_{3} e^{i \bar{k} \tilde{x}_{3}} d\left(\tilde{x}_{3}\right) e^{s_{X ; r}\left(t_{3}, t_{4}, \tilde{x}_{3}\right)} \int_{-\infty}^{\infty} d \tilde{x}_{4} e^{-i \bar{k} \tilde{x}_{4}} d\left(\tilde{x}_{4}\right) e^{s_{X ; r}^{*}\left(t_{4}, t_{3}, \tilde{x}_{4}\right)} \\
& \times \int_{-\infty}^{\infty} d y\left\{e^{S_{X ; r r}\left(t_{1}, t_{3}, t_{2}, t_{4} ; y ; \tilde{x}_{1}, \tilde{x}_{3}, y\right)} e^{S_{X ; r r}^{*}\left(t_{4}, t_{2}, t_{3}, t_{1} ; y ; \tilde{x}_{4}, \tilde{x}_{2},-y\right)}-1\right\}
\end{aligned}
$$

and

$$
\begin{aligned}
& \left\langle\left\langle\tilde{P}_{r}\left(t_{1}\right) \tilde{P}_{r}^{\dagger}\left(t_{2}\right) \tilde{P}_{r}\left(t_{3}\right) \tilde{P}_{r}^{\dagger}\left(t_{4}\right)\right\rangle\right\rangle_{c}^{I I}=e^{-i\left(t_{1}-t_{2}+t_{3}-t_{4}\right)\left(E_{h}-\varepsilon(0)\right)} \\
& \times \int_{0}^{\infty} \frac{d k}{2 \pi} e^{i k v_{f}\left(t_{4}-t_{1}\right)} \int_{-\infty}^{\infty} d \tilde{x}_{1} e^{i k \tilde{x}_{1}} d\left(\tilde{x}_{1}\right) e^{s_{X ; r}\left(t_{1}, t_{2}, \tilde{x}_{1}\right)} \int_{-\infty}^{\infty} d \tilde{x}_{4} e^{-i k \tilde{x}_{4}} d\left(\tilde{x}_{4}\right) e^{s_{X ; r}^{*}\left(t_{4}, t_{3}, \tilde{x}_{4}\right)} \\
& \times \int_{0}^{\infty} \frac{d \bar{k}}{2 \pi} e^{i \bar{k} v_{f}\left(t_{3}-t_{2}\right)} \int_{-\infty}^{\infty} d \tilde{x}_{3} e^{i \bar{k} \tilde{x}_{2}} d\left(\tilde{x}_{3}\right) e^{s_{X ; r}\left(t_{3}, t_{4}, \tilde{x}_{3}\right)} \int_{-\infty}^{\infty} d \tilde{x}_{2} e^{-i \bar{k} \tilde{x}_{3}} d\left(\tilde{x}_{2}\right) e^{s_{X ; r}^{*}\left(t_{2}, t_{1}, \tilde{x}_{2}\right)} \\
& \times \int_{-\infty}^{\infty} d y e^{i(k+\bar{k}) y} e^{S_{X ; r r}\left(t_{1}, t_{3}, t_{2}, t_{4} ; y ; \tilde{x}_{1}, \tilde{x}_{3}, y\right)} e^{S_{X ; r r}^{*}\left(t_{4}, t_{2}, t_{3}, t_{1} ; y ; \tilde{x}_{4}, \tilde{x}_{2},-y\right)} .
\end{aligned}
$$

The main difference between them consists in the form of the integrals with respect to the variable $y$ which can be identified as the relative distance between the two valence holes which are created by the two polarization operators $P_{r}^{\dagger}\left(t_{2}\right)$ and $P_{r}^{\dagger}\left(t_{4}\right)$.

The integral with respect to $y$ in the first contribution is independent of the wave vectors $k$ and $\bar{k}$ which are related to the free electronic two-point functions appearing in Equation C.7. In addition to that, the integrand contains a term -1 which comes from the product of two-point response functions which is subtracted from the total four-point response function when calculating the connected part. This term is crucial 
for the convergence of the integral since the behavior of the first term in the integrand for large positive or negative values of $y$ is described by the relation

$$
\lim _{y \rightarrow \pm \infty} e^{S_{X ; r r}\left(t_{1}, t_{3}, t_{2}, t_{4} ; ; ; \tilde{x}_{1}, \tilde{x}_{3}, y\right)} e^{S_{X ; r r}^{*}\left(t_{4}, t_{2}, t_{3}, t_{1} ; y ; \tilde{x}_{4}, \tilde{x}_{2},-y\right)}=1+\mathcal{O}\left(\frac{1}{y^{2}}\right) .
$$

The integral with respect to $y$ in the second contribution depends on the wave vectors $k$ and $\bar{k}$ because of the factor $e^{i(k+\bar{k}) y}$ which ensures the convergence of the integral. If the particles do not interact with each other, both integrals vanish and the total response function only consists of the disconnected part describing the successive creation and annihilation of two pairs of free right moving electrons and valence holes.

The different character of the two contributions is a consequence of the fact that the field operators of the particles can be paired in two different ways. The first contribution corresponds to the case in which field operators of electrons and holes which have the same time in common are always paired with field operators whose times are also identical. If, for example, $\psi_{h ; 0}\left(y_{1}, t_{1}\right)$ is paired with $\psi_{h ; 0}^{\dagger}\left(y_{2}, t_{2}\right)$, the operator $\psi_{e r ; 0}\left(x_{1}, t_{1}\right)$ has to be paired with $\psi_{e r ; 0}^{\dagger}\left(x_{2}, t_{2}\right)$. The second contribution corresponds to the case in which field operators with the same time are paired with field operators whose times are not identical.

The other connected four-point response functions are composed of contributions which are similar to the two contributions which have already been discussed. In the case of the excitonic response functions which are related to the spatial correlation functions in Equations C.9 and C.10 the following relations

$$
\lim _{L \rightarrow \infty} \frac{1}{L}\left\langle\Omega_{0}\left|\tilde{P}_{r}\left(t_{1}\right) \tilde{P}_{r}^{\dagger}\left(t_{2}\right) \tilde{P}_{l}\left(t_{3}\right) \tilde{P}_{l}^{\dagger}\left(t_{4}\right)\right| \Omega_{0}\right\rangle_{c}=\left\langle\left\langle\tilde{P}_{r}\left(t_{1}\right) \tilde{P}_{r}^{\dagger}\left(t_{2}\right) \tilde{P}_{l}\left(t_{3}\right) \tilde{P}_{l}^{\dagger}\left(t_{4}\right)\right\rangle\right\rangle_{c}
$$

and

$$
\lim _{L \rightarrow \infty} \frac{1}{L}\left\langle\Omega_{0}\left|\tilde{P}_{r}\left(t_{1}\right) \tilde{P}_{l}^{\dagger}\left(t_{2}\right) \tilde{P}_{l}\left(t_{3}\right) \tilde{P}_{r}^{\dagger}\left(t_{4}\right)\right| \Omega_{0}\right\rangle_{c}=\left\langle\left\langle\tilde{P}_{r}\left(t_{1}\right) \tilde{P}_{l}^{\dagger}\left(t_{2}\right) \tilde{P}_{l}\left(t_{3}\right) \tilde{P}_{r}^{\dagger}\left(t_{4}\right)\right\rangle\right\rangle_{c}
$$

with

$$
\begin{aligned}
& \left\langle\left\langle\tilde{P}_{r}\left(t_{1}\right) \tilde{P}_{r}^{\dagger}\left(t_{2}\right) \tilde{P}_{l}\left(t_{3}\right) \tilde{P}_{l}^{\dagger}\left(t_{4}\right)\right\rangle\right\rangle_{c}=e^{-i\left(t_{1}-t_{2}+t_{3}-t_{4}\right)\left(E_{h}-\varepsilon(0)\right)} \\
& \times \int_{0}^{\infty} \frac{d k}{2 \pi} e^{i k v_{f}\left(t_{2}-t_{1}\right)} \int_{-\infty}^{\infty} d \tilde{x}_{1} e^{i k \tilde{x}_{1}} d\left(\tilde{x}_{1}\right) e^{s_{X ; r}\left(t_{1}, t_{2}, \tilde{x}_{1}\right)} \int_{-\infty}^{\infty} d \tilde{x}_{2} e^{-i k \tilde{x}_{2}} d\left(\tilde{x}_{2}\right) e^{s_{X ; r}^{*}\left(t_{2}, t_{1}, \tilde{x}_{2}\right)} \\
& \times \int_{0}^{\infty} \frac{d \bar{k}}{2 \pi} e^{i \bar{k} v_{f}\left(t_{4}-t_{3}\right)} \int_{-\infty}^{\infty} d \tilde{x}_{3} e^{-i \bar{k} \tilde{x}_{3}} d\left(\tilde{x}_{3}\right) e^{s_{X ; l}\left(t_{3}, t_{4}, \tilde{x}_{3}\right)} \int_{-\infty}^{\infty} d \tilde{x}_{4} e^{i \bar{k} \tilde{x}_{4}} d\left(\tilde{x}_{4}\right) e^{s_{X ; l}^{*}\left(t_{4}, t_{3}, \tilde{x}_{4}\right)} \\
& \times \int_{-\infty}^{\infty} d y\left\{e^{S_{X ; r l}\left(t_{1}, t_{3}, t_{2}, t_{4} ; y ; \tilde{x}_{1}, \tilde{x}_{3}, y\right)} e^{S_{X ; l r}^{*}\left(t_{4}, t_{2}, t_{3}, t_{1} ; y ; \tilde{x}_{4}, \tilde{x}_{2},-y\right)}-1\right\}
\end{aligned}
$$


and

$$
\begin{aligned}
& \left\langle\left\langle\tilde{P}_{r}\left(t_{1}\right) \tilde{P}_{l}^{\dagger}\left(t_{2}\right) \tilde{P}_{l}\left(t_{3}\right) \tilde{P}_{r}^{\dagger}\left(t_{4}\right)\right\rangle\right\rangle_{c}=e^{-i\left(t_{1}-t_{2}+t_{3}-t_{4}\right)\left(E_{h}-\varepsilon(0)\right)} \\
& \times \int_{0}^{\infty} \frac{d k}{2 \pi} e^{i k v_{f}\left(t_{4}-t_{1}\right)} \int_{-\infty}^{\infty} d \tilde{x}_{1} e^{i k \tilde{x}_{1}} d\left(\tilde{x}_{1}\right) e^{s_{X ; r}\left(t_{1}, t_{2}, \tilde{x}_{1}\right)} \int_{-\infty}^{\infty} d \tilde{x}_{4} e^{-i k \tilde{x}_{4}} d\left(\tilde{x}_{4}\right) e^{s_{X ; r}^{*}\left(t_{4}, t_{3}, \tilde{x}_{4}\right)} \\
& \times \int_{0}^{\infty} \frac{d \bar{k}}{2 \pi} e^{i \bar{k} v_{f}\left(t_{3}-t_{2}\right)} \int_{-\infty}^{\infty} d \tilde{x}_{3} e^{i \bar{k} \tilde{x}_{3}} d\left(\tilde{x}_{3}\right) e^{s_{X ; l}\left(t_{3}, t_{4}, \tilde{x}_{3}\right)} \int_{-\infty}^{\infty} d \tilde{x}_{2} e^{-i \bar{k} \tilde{x}_{2}} d\left(\tilde{x}_{2}\right) e^{s_{X ; l}^{*}\left(t_{2}, t_{1}, \tilde{x}_{2}\right)} \\
& \times \int_{-\infty}^{\infty} d y e^{i\left(2 k_{f}+k-\bar{k}\right) y} e^{S_{X ; r l}\left(t_{1}, t_{3}, t_{2}, t_{4} ; y ; \tilde{x}_{1}, \tilde{x}_{3}, y\right)} e^{S_{X ; r l}^{*}\left(t_{4}, t_{2}, t_{3}, t_{1} ; y ; \tilde{x}_{4}, \tilde{x}_{2},-y\right)}
\end{aligned}
$$

are obtained. The term on the right-hand side of Equation C.23 resembles the first contribution in Equation C. 19 while the response function in Equation C.24 is similar to the second contribution of Equation C.19 with the factor $e^{i\left(2 k_{f}+k-\bar{k}\right) y}$ instead of $e^{i(k+\bar{k}) y}$. If the particles are assumed to be free, the connected excitonic response function in Equation C.23 equals zero whereas the response function in Equation C.24 yields a non-vanishing contribution. The appearance of the wave vector $2 k_{f}$ can then be explained within the framework of a simple one-particle picture since the free version of the connected response function in Equation C.24 describes the annihilation and creation of left moving electrons in the Fermi sea whose distance to the Fermi point of the left movers is larger than $2 k_{f}$.

The connected part of the biexcitonic response function on the left-hand side of Equation C.4 is calculated with the help of the spatial correlation function in Equation C.13. The result is given by

$$
\begin{aligned}
& \lim _{L \rightarrow \infty} \frac{1}{L}\left\langle\Omega_{0}\left|\tilde{P}_{r}\left(t_{1}\right) \tilde{P}_{r}\left(t_{2}\right) \tilde{P}_{r}^{\dagger}\left(t_{3}\right) \tilde{P}_{r}^{\dagger}\left(t_{4}\right)\right| \Omega_{0}\right\rangle_{c}=\left\langle\left\langle\tilde{P}_{r}\left(t_{1}\right) \tilde{P}_{r}\left(t_{2}\right) \tilde{P}_{r}^{\dagger}\left(t_{3}\right) \tilde{P}_{r}^{\dagger}\left(t_{4}\right)\right\rangle\right\rangle_{c} \\
& =\left\langle\left\langle\tilde{P}_{r}\left(t_{1}\right) \tilde{P}_{r}\left(t_{2}\right) \tilde{P}_{r}^{\dagger}\left(t_{3}\right) \tilde{P}_{r}^{\dagger}\left(t_{4}\right)\right\rangle\right\rangle_{c}^{I}+\left\langle\left\langle\tilde{P}_{r}\left(t_{1}\right) \tilde{P}_{r}\left(t_{2}\right) \tilde{P}_{r}^{\dagger}\left(t_{3}\right) \tilde{P}_{r}^{\dagger}\left(t_{4}\right)\right\rangle\right\rangle_{c}^{I I} \\
& +\left\langle\left\langle\tilde{P}_{r}\left(t_{1}\right) \tilde{P}_{r}\left(t_{2}\right) \tilde{P}_{r}^{\dagger}\left(t_{3}\right) \tilde{P}_{r}^{\dagger}\left(t_{4}\right)\right\rangle\right\rangle_{c}^{I I I}+\left\langle\left\langle\tilde{P}_{r}\left(t_{1}\right) \tilde{P}_{r}\left(t_{2}\right) \tilde{P}_{r}^{\dagger}\left(t_{3}\right) \tilde{P}_{r}^{\dagger}\left(t_{4}\right)\right\rangle\right\rangle_{c}^{I V} \cdot
\end{aligned}
$$

The first two contributions on the right-hand side of Equation C.27, which are defined by the identities

$$
\begin{aligned}
& \left\langle\left\langle\tilde{P}_{r}\left(t_{1}\right) \tilde{P}_{r}\left(t_{2}\right) \tilde{P}_{r}^{\dagger}\left(t_{3}\right) \tilde{P}_{r}^{\dagger}\left(t_{4}\right)\right\rangle\right\rangle_{c}^{I}=e^{-i\left(t_{1}+t_{2}-t_{3}-t_{4}\right)\left(E_{h}-\varepsilon(0)\right)} \\
& \times \int_{0}^{\infty} \frac{d k}{2 \pi} e^{i k v_{f}\left(t_{4}-t_{1}\right)} \int_{-\infty}^{\infty} d \tilde{x}_{1} e^{i k \tilde{x}_{1}} d\left(\tilde{x}_{1}\right) e^{s_{X ; r}\left(t_{1}, t_{4}, \tilde{x}_{1}\right)} \int_{-\infty}^{\infty} d \tilde{x}_{4} e^{-i k \tilde{x}_{4}} d\left(\tilde{x}_{4}\right) e^{s_{X ; r}^{*}\left(t_{4}, t_{1}, \tilde{x}_{4}\right)} \\
& \times \int_{0}^{\infty} \frac{d \bar{k}}{2 \pi} e^{i \bar{k} v_{f}\left(t_{3}-t_{2}\right)} \int_{-\infty}^{\infty} d \tilde{x}_{2} e^{i \bar{k} \tilde{x}_{2}} d\left(\tilde{x}_{2}\right) e^{s_{X ; r}\left(t_{2}, t_{3}, \tilde{x}_{2}\right)} \int_{-\infty}^{\infty} d \tilde{x}_{3} e^{-i \bar{k} \tilde{x}_{3}} d\left(\tilde{x}_{3}\right) e^{s_{X ; r}^{*}\left(t_{3}, t_{2}, \tilde{x}_{3}\right)} \\
& \times \int_{-\infty}^{\infty} d y\left\{e^{i\left(t_{2}-t_{3}\right) 2 \varepsilon(y)} e^{S_{X X ; r r}\left(t_{1}, t_{2}, t_{4}, t_{3} ; y ; \tilde{x}_{1}, \tilde{x}_{2}, y\right)} e^{S_{X X ; r r}^{*}\left(t_{4}, t_{3}, t_{1}, t_{2} ; y ; \tilde{x}_{4}, \tilde{x}_{3}, y\right)}-1\right\}
\end{aligned}
$$


and

$$
\begin{aligned}
& \left\langle\left\langle\tilde{P}_{r}\left(t_{1}\right) \tilde{P}_{r}\left(t_{2}\right) \tilde{P}_{r}^{\dagger}\left(t_{3}\right) \tilde{P}_{r}^{\dagger}\left(t_{4}\right)\right\rangle\right\rangle_{c}^{I I}=e^{-i\left(t_{1}+t_{2}-t_{3}-t_{4}\right)\left(E_{h}-\varepsilon(0)\right)} \\
& \times \int_{0}^{\infty} \frac{d k}{2 \pi} e^{i k v_{f}\left(t_{3}-t_{1}\right)} \int_{-\infty}^{\infty} d \tilde{x}_{1} e^{i k \tilde{x}_{1}} d\left(\tilde{x}_{1}\right) e^{s_{X ; r}\left(t_{1}, t_{3}, \tilde{x}_{1}\right)} \int_{-\infty}^{\infty} d \tilde{x}_{3} e^{-i k \tilde{x}_{3}} d\left(\tilde{x}_{3}\right) e^{s_{X ; r}^{*}\left(t_{3}, t_{1}, \tilde{x}_{3}\right)} \\
& \times \int_{0}^{\infty} \frac{d \bar{k}}{2 \pi} e^{i \bar{k} v_{f}\left(t_{4}-t_{2}\right)} \int_{-\infty}^{\infty} d \tilde{x}_{2} e^{i \bar{k} \tilde{x}_{2}} d\left(\tilde{x}_{2}\right) e^{s_{X ; r}\left(t_{2}, t_{4}, \tilde{x}_{2}\right)} \int_{-\infty}^{\infty} d \tilde{x}_{4} e^{-i \bar{k} \tilde{x}_{4}} d\left(\tilde{x}_{4}\right) e^{s_{X ; r}^{*}\left(t_{4}, t_{2}, \tilde{x}_{4}\right)} \\
& \times \int_{-\infty}^{\infty} d y\left\{e^{i\left(t_{2}-t_{3}\right) 2 \varepsilon(y)} e^{S_{X X ; r r}\left(t_{1}, t_{2}, t_{3}, t_{4} ; y ; \tilde{x}_{1}, \tilde{x}_{2}, y\right)} e^{S_{X X ; r r}^{*}\left(t_{4}, t_{3}, t_{2}, t_{1} ; y ; \tilde{x}_{4}, \tilde{x}_{3},-y\right)}-1\right\},
\end{aligned}
$$

exhibit the same structure as the first contribution in Equation C.19 whereas the third and the fourth contribution, which satisfy the relations

$$
\begin{aligned}
& \left\langle\left\langle\tilde{P}_{r}\left(t_{1}\right) \tilde{P}_{r}\left(t_{2}\right) \tilde{P}_{r}^{\dagger}\left(t_{3}\right) \tilde{P}_{r}^{\dagger}\left(t_{4}\right)\right\rangle\right\rangle_{c}^{I I I}=-e^{-i\left(t_{1}+t_{2}-t_{3}-t_{4}\right)\left(E_{h}-\varepsilon(0)\right)} \\
& \times \int_{0}^{\infty} \frac{d k}{2 \pi} e^{i k v_{f}\left(t_{3}-t_{1}\right)} \int_{-\infty}^{\infty} d \tilde{x}_{1} e^{i k \tilde{x}_{1}} d\left(\tilde{x}_{1}\right) e^{s_{X ; r}\left(t_{1}, t_{4}, \tilde{x}_{1}\right)} \int_{-\infty}^{\infty} d \tilde{x}_{3} e^{-i k \tilde{x}_{3}} d\left(\tilde{x}_{3}\right) e^{s_{X ; r}^{*}\left(t_{3}, t_{2}, \tilde{x}_{3}\right)} \\
& \times \int_{0}^{\infty} \frac{d \bar{k}}{2 \pi} e^{i \bar{k} v_{f}\left(t_{4}-t_{2}\right)} \int_{-\infty}^{\infty} d \tilde{x}_{2} e^{i \bar{k} \tilde{x}_{2}} d\left(\tilde{x}_{2}\right) e^{s_{X ; r}\left(t_{2}, t_{3}, \tilde{x}_{2}\right)} \int_{-\infty}^{\infty} d \tilde{x}_{4} e^{-i \bar{k} \tilde{x}_{4}} d\left(\tilde{x}_{4}\right) e^{s_{X ; r}^{*}\left(t_{4}, t_{1}, \tilde{x}_{4}\right)} \\
& \times \int_{-\infty}^{\infty} d y e^{i(k-\bar{k}) y} e^{i\left(t_{2}-t_{3}\right) 2 \varepsilon(y)} e^{S_{X X ; r r}\left(t_{1}, t_{2}, t_{4}, t_{3} ; y ; \tilde{x}_{1}, \tilde{x}_{2}, y\right)} e^{S_{X X ; r r}^{*}\left(t_{4}, t_{3}, t_{1}, t_{2} ; y ; \tilde{x}_{4}, \tilde{x}_{3}, y\right)} \quad \text { (C.30) }
\end{aligned}
$$

and

$$
\begin{aligned}
& \left\langle\left\langle\tilde{P}_{r}\left(t_{1}\right) \tilde{P}_{r}\left(t_{2}\right) \tilde{P}_{r}^{\dagger}\left(t_{3}\right) \tilde{P}_{r}^{\dagger}\left(t_{4}\right)\right\rangle\right\rangle_{c}^{I V}=-e^{-i\left(t_{1}+t_{2}-t_{3}-t_{4}\right)\left(E_{h}-\varepsilon(0)\right)} \\
& \times \int_{0}^{\infty} \frac{d k}{2 \pi} e^{i k v_{f}\left(t_{4}-t_{1}\right)} \int_{-\infty}^{\infty} d \tilde{x}_{1} e^{i k \tilde{x}_{1}} d\left(\tilde{x}_{1}\right) e^{s_{X ; r}\left(t_{1}, t_{3}, \tilde{x}_{1}\right)} \int_{-\infty}^{\infty} d \tilde{x}_{4} e^{-i k \tilde{x}_{4}} d\left(\tilde{x}_{4}\right) e^{s_{X ; r}^{*}\left(t_{4}, t_{2}, \tilde{x}_{4}\right)} \\
& \times \int_{0}^{\infty} \frac{d \bar{k}}{2 \pi} e^{i \bar{k} v_{f}\left(t_{3}-t_{2}\right)} \int_{-\infty}^{\infty} d \tilde{x}_{2} e^{i \bar{k} \tilde{x}_{2}} d\left(\tilde{x}_{2}\right) e^{s_{X ; r}\left(t_{2}, t_{4}, \tilde{x}_{2}\right)} \int_{-\infty}^{\infty} d \tilde{x}_{3} e^{-i \bar{k} \tilde{x}_{3}} d\left(\tilde{x}_{3}\right) e^{s_{X ; r}^{*}\left(t_{3}, t_{1}, \tilde{x}_{3}\right)} \\
& \times \int_{-\infty}^{\infty} d y e^{i(k-\bar{k}) y} e^{i\left(t_{2}-t_{3}\right) 2 \varepsilon(y)} e^{S_{X X ; r r}\left(t_{1}, t_{2}, t_{3}, t_{4} ; y ; \tilde{x}_{1}, \tilde{x}_{2}, y\right)} e^{S_{X X ; r r}^{*}\left(t_{4}, t_{3}, t_{2}, t_{1} ; y ; \tilde{x}_{4}, \tilde{x}_{3},-y\right)},
\end{aligned}
$$

are structured in the same way as the second contribution in Equation C.19. If the particles do not interact with each other, the biexcitonic response function does not vanish unlike the corresponding excitonic response function.

Finally, the biexcitonic response functions which are associated with the spatial correlation functions in Equations C.15 and C.16 satisfy the relations

$$
\begin{aligned}
& \lim _{L \rightarrow \infty} \frac{1}{L}\left\langle\Omega_{0}\left|\tilde{P}_{r}\left(t_{1}\right) \tilde{P}_{l}\left(t_{2}\right) \tilde{P}_{r}^{\dagger}\left(t_{3}\right) \tilde{P}_{l}^{\dagger}\left(t_{4}\right)\right| \Omega_{0}\right\rangle_{c}=\left\langle\left\langle\tilde{P}_{r}\left(t_{1}\right) \tilde{P}_{l}\left(t_{2}\right) \tilde{P}_{r}^{\dagger}\left(t_{3}\right) \tilde{P}_{l}^{\dagger}\left(t_{4}\right)\right\rangle\right\rangle_{c} \\
& =\left\langle\left\langle\tilde{P}_{r}\left(t_{1}\right) \tilde{P}_{l}\left(t_{2}\right) \tilde{P}_{r}^{\dagger}\left(t_{3}\right) \tilde{P}_{l}^{\dagger}\left(t_{4}\right)\right\rangle\right\rangle_{c}^{I}+\left\langle\left\langle\tilde{P}_{r}\left(t_{1}\right) \tilde{P}_{l}\left(t_{2}\right) \tilde{P}_{r}^{\dagger}\left(t_{3}\right) \tilde{P}_{l}^{\dagger}\left(t_{4}\right)\right\rangle\right\rangle_{c}^{I I}
\end{aligned}
$$


and

$$
\begin{aligned}
& \lim _{L \rightarrow \infty} \frac{1}{L}\left\langle\Omega_{0}\left|\tilde{P}_{r}\left(t_{1}\right) \tilde{P}_{l}\left(t_{2}\right) \tilde{P}_{l}^{\dagger}\left(t_{3}\right) \tilde{P}_{r}^{\dagger}\left(t_{4}\right)\right| \Omega_{0}\right\rangle_{c}=\left\langle\left\langle\tilde{P}_{r}\left(t_{1}\right) \tilde{P}_{l}\left(t_{2}\right) \tilde{P}_{l}^{\dagger}\left(t_{3}\right) \tilde{P}_{r}^{\dagger}\left(t_{4}\right)\right\rangle\right\rangle_{c} \\
& =\left\langle\left\langle\tilde{P}_{r}\left(t_{1}\right) \tilde{P}_{l}\left(t_{2}\right) \tilde{P}_{l}^{\dagger}\left(t_{3}\right) \tilde{P}_{r}^{\dagger}\left(t_{4}\right)\right\rangle\right\rangle_{c}^{I}+\left\langle\left\langle\tilde{P}_{r}\left(t_{1}\right) \tilde{P}_{l}\left(t_{2}\right) \tilde{P}_{l}^{\dagger}\left(t_{3}\right) \tilde{P}_{r}^{\dagger}\left(t_{4}\right)\right\rangle\right\rangle_{c}^{I I} .
\end{aligned}
$$

The first contributions in both equations, which are defined by the relations

$$
\begin{aligned}
& \left\langle\left\langle\tilde{P}_{r}\left(t_{1}\right) \tilde{P}_{l}\left(t_{2}\right) \tilde{P}_{r}^{\dagger}\left(t_{3}\right) \tilde{P}_{l}^{\dagger}\left(t_{4}\right)\right\rangle\right\rangle_{c}^{I}=e^{-i\left(t_{1}+t_{2}-t_{3}-t_{4}\right)\left(E_{h}-\varepsilon(0)\right)} \\
& \times \int_{0}^{\infty} \frac{d k}{2 \pi} e^{i k v_{f}\left(t_{3}-t_{1}\right)} \int_{-\infty}^{\infty} d \tilde{x}_{1} e^{i k \tilde{x}_{1}} d\left(\tilde{x}_{1}\right) e^{s_{X ; r}\left(t_{1}, t_{3}, \tilde{x}_{1}\right)} \int_{-\infty}^{\infty} d \tilde{x}_{3} e^{-i k \tilde{x}_{3}} d\left(\tilde{x}_{3}\right) e^{s_{X ; r}^{*}\left(t_{3}, t_{1}, \tilde{x}_{3}\right)} \\
& \times \int_{0}^{\infty} \frac{d \bar{k}}{2 \pi} e^{i \bar{k} v_{f}\left(t_{4}-t_{2}\right)} \int_{-\infty}^{\infty} d \tilde{x}_{2} e^{-i \bar{k} \tilde{x}_{2}} d\left(\tilde{x}_{2}\right) e^{s_{X ; l}\left(t_{2}, t_{4}, \tilde{x}_{2}\right)} \int_{-\infty}^{\infty} d \tilde{x}_{4} e^{i \bar{k} \tilde{x}_{4}} d\left(\tilde{x}_{4}\right) e^{s_{X ; l}^{*}\left(t_{4}, t_{2}, \tilde{x}_{4}\right)} \\
& \times \int_{-\infty}^{\infty} d y\left\{e^{i\left(t_{2}-t_{3}\right) 2 \varepsilon(y)} e^{S_{X X ; r l}\left(t_{1}, t_{2}, t_{3}, t_{4} ; y ; \tilde{x}_{1}, \tilde{x}_{2}, y\right)} e^{S_{X X ; l r}^{*}\left(t_{4}, t_{3}, t_{2}, t_{1} ; y ; \tilde{x}_{4}, \tilde{x}_{3},-y\right)}-1\right\}
\end{aligned}
$$

and

$$
\begin{aligned}
& \left\langle\left\langle\tilde{P}_{r}\left(t_{1}\right) \tilde{P}_{l}\left(t_{2}\right) \tilde{P}_{l}^{\dagger}\left(t_{3}\right) \tilde{P}_{r}^{\dagger}\left(t_{4}\right)\right\rangle\right\rangle_{c}^{I}=e^{-i\left(t_{1}+t_{2}-t_{3}-t_{4}\right)\left(E_{h}-\varepsilon(0)\right)} \\
& \times \int_{0}^{\infty} \frac{d k}{2 \pi} e^{i k v_{f}\left(t_{4}-t_{1}\right)} \int_{-\infty}^{\infty} d \tilde{x}_{1} e^{i k \tilde{x}_{1}} d\left(\tilde{x}_{1}\right) e^{s_{X ; r}\left(t_{1}, t_{4}, \tilde{x}_{1}\right)} \int_{-\infty}^{\infty} d \tilde{x}_{4} e^{-i k \tilde{x}_{4}} d\left(\tilde{x}_{4}\right) e^{s_{X ; r}^{*}\left(t_{4}, t_{1}, \tilde{x}_{4}\right)} \\
& \times \int_{0}^{\infty} \frac{d \bar{k}}{2 \pi} e^{i \bar{k} v_{f}\left(t_{3}-t_{2}\right)} \int_{-\infty}^{\infty} d \tilde{x}_{2} e^{-i \bar{k} \tilde{x}_{2}} d\left(\tilde{x}_{2}\right) e^{s_{X ; l}\left(t_{2}, t_{3}, \tilde{x}_{2}\right)} \int_{-\infty}^{\infty} d \tilde{x}_{3} e^{i \bar{k} \tilde{x}_{3}} d\left(\tilde{x}_{3}\right) e^{s_{X ; l}^{*}\left(t_{3}, t_{2}, \tilde{x}_{3}\right)} \\
& \times \int_{-\infty}^{\infty} d y\left\{e^{i\left(t_{2}-t_{3}\right) 2 \varepsilon(y)} e^{S_{X X ; r l}\left(t_{1}, t_{2}, t_{4}, t_{3} ; y ; \tilde{x}_{1}, \tilde{x}_{2}, y\right)} e^{S_{X X ; r l}^{*}\left(t_{4}, t_{3}, t_{1}, t_{2} ; y ; \tilde{x}_{4}, \tilde{x}_{3}, y\right)}-1\right\},
\end{aligned}
$$

resemble the excitonic response function in Equation C.23 whereas the second contributions in Equations C.32 and C.33, which satisfy the equations

$$
\begin{aligned}
& \left\langle\left\langle\tilde{P}_{r}\left(t_{1}\right) \tilde{P}_{l}\left(t_{2}\right) \tilde{P}_{r}^{\dagger}\left(t_{3}\right) \tilde{P}_{l}^{\dagger}\left(t_{4}\right)\right\rangle\right\rangle_{c}^{I I}=-e^{-i\left(t_{1}+t_{2}-t_{3}-t_{4}\right)\left(E_{h}-\varepsilon(0)\right)} \\
& \times \int_{0}^{\infty} \frac{d k}{2 \pi} e^{i k v_{f}\left(t_{3}-t_{1}\right)} \int_{-\infty}^{\infty} d \tilde{x}_{1} e^{i k \tilde{x}_{1}} d\left(\tilde{x}_{1}\right) e^{s_{X ; r}\left(t_{1}, t_{4}, \tilde{x}_{1}\right)} \int_{-\infty}^{\infty} d \tilde{x}_{3} e^{-i k \tilde{x}_{3}} d\left(\tilde{x}_{3}\right) e^{s_{X ; r}^{*}\left(t_{3}, t_{2}, \tilde{x}_{3}\right)} \\
& \times \int_{0}^{\infty} \frac{d \bar{k}}{2 \pi} e^{i \bar{k} v_{f}\left(t_{4}-t_{2}\right)} \int_{-\infty}^{\infty} d \tilde{x}_{2} e^{-i \bar{k} \tilde{x}_{2}} d\left(\tilde{x}_{2}\right) e^{s_{X ; l}\left(t_{2}, t_{3}, \tilde{x}_{2}\right)} \int_{-\infty}^{\infty} d \tilde{x}_{4} e^{i \bar{k} \tilde{x}_{4}} d\left(\tilde{x}_{4}\right) e^{s_{X ; l}^{*}\left(t_{4}, t_{1}, \tilde{x}_{4}\right)} \\
& \times \int_{-\infty}^{\infty} d y e^{i\left(2 k_{f}+k+\bar{k}\right) y} e^{i\left(t_{2}-t_{3}\right) 2 \varepsilon(y)} e^{S_{X X ; r l}\left(t_{1}, t_{2}, t_{4}, t_{3} ; y ; \tilde{x}_{1}, \tilde{x}_{2}, y\right)} e^{S_{X X ; l r}^{*}\left(t_{4}, t_{3}, t_{1}, t_{2} ; y ; \tilde{x}_{4}, \tilde{x}_{3}, y\right)}
\end{aligned}
$$


and

$$
\begin{aligned}
& \left\langle\left\langle\tilde{P}_{r}\left(t_{1}\right) \tilde{P}_{l}\left(t_{2}\right) \tilde{P}_{l}^{\dagger}\left(t_{3}\right) \tilde{P}_{r}^{\dagger}\left(t_{4}\right)\right\rangle\right\rangle_{c}^{I I}=-e^{-i\left(t_{1}+t_{2}-t_{3}-t_{4}\right)\left(E_{h}-\varepsilon(0)\right)} \\
& \times \int_{0}^{\infty} \frac{d k}{2 \pi} e^{i k v_{f}\left(t_{4}-t_{1}\right)} \int_{-\infty}^{\infty} d \tilde{x}_{1} e^{i k \tilde{x}_{1}} d\left(\tilde{x}_{1}\right) e^{s_{X ; r}\left(t_{1}, t_{3}, \tilde{x}_{1}\right)} \int_{-\infty}^{\infty} d \tilde{x}_{4} e^{-i k \tilde{x}_{4}} d\left(\tilde{x}_{4}\right) e^{s_{X ; r}^{*}\left(t_{4}, t_{2}, \tilde{x}_{4}\right)} \\
& \times \int_{0}^{\infty} \frac{d \bar{k}}{2 \pi} e^{i \bar{k} v_{f}\left(t_{3}-t_{2}\right)} \int_{-\infty}^{\infty} d \tilde{x}_{2} e^{-i \bar{k} \tilde{x}_{2}} d\left(\tilde{x}_{2}\right) e^{s_{X ; l}\left(t_{2}, t_{4}, \tilde{x}_{2}\right)} \int_{-\infty}^{\infty} d \tilde{x}_{3} e^{i \bar{k} \tilde{x}_{3}} d\left(\tilde{x}_{3}\right) e^{s_{X ; l}^{*}\left(t_{3}, t_{1}, \tilde{x}_{3}\right)} \\
& \times \int_{-\infty}^{\infty} d y e^{i\left(2 k_{f}+k+\bar{k}\right) y} e^{i\left(t_{2}-t_{3}\right) 2 \varepsilon(y)} e^{S_{X X ; r l}\left(t_{1}, t_{2}, t_{3}, t_{4} ; y ; \tilde{x}_{1}, \tilde{x}_{2}, y\right)} e^{S_{X X ; r l}^{*}\left(t_{4}, t_{3}, t_{2}, t_{1} ; y ; \tilde{x}_{4}, \tilde{x}_{3},-y\right)},
\end{aligned}
$$

are similar to the excitonic response function in Equation C.24. If the particles do not interact with each other, all contributions vanish and the biexcitonic response functions in Equations C.32 and C.33 are equal to zero.

\section{C.2 Approximations}

The exact calculation of the four-point response functions turns out to be a difficult task since it requires the evaluation of seven-dimensional integrals. If, however, the auxiliary functions vary slowly on the length scale which is determined by the width of the Lorentzian curve $\Lambda$, the same approximation which has already been used to simplify the two-point response functions in Section 3.4 can be applied to eliminate the integrations with respect to the variables $\tilde{x}_{i}$.

In the case of the excitonic response function of Equation C.19 this approximation leads to the following expression

$$
\begin{aligned}
& \left\langle\left\langle\tilde{P}_{r}\left(t_{1}\right) \tilde{P}_{r}^{\dagger}\left(t_{2}\right) \tilde{P}_{r}\left(t_{3}\right) \tilde{P}_{r}^{\dagger}\left(t_{4}\right)\right\rangle\right\rangle_{c} \\
& \approx e^{-i\left(t_{1}-t_{2}+t_{3}-t_{4}\right)\left(E_{h}-\varepsilon(0)\right)} e^{s_{X ; r}\left(t_{1}, t_{2}, 0\right)} e^{s_{X ; r}^{*}\left(t_{2}, t_{1}, 0\right)} e^{s_{X ; r}\left(t_{3}, t_{4}, 0\right)} e^{s_{X ; r}^{*}\left(t_{4}, t_{3}, 0\right)} \\
& \quad \times(2 \pi i)^{-2}\left(v_{f}\left(t_{1}-t_{2}\right)-i 2 \Lambda\right)^{-1}\left(v_{f}\left(t_{3}-t_{4}\right)-i 2 \Lambda\right)^{-1} \\
& \quad \times \int_{-\infty}^{\infty} d y\left\{e^{S_{X ; r r}\left(t_{1}, t_{3}, t_{2}, t_{4} ; y ; 0,0, y\right)} e^{S_{X ; r r}^{*}\left(t_{4}, t_{2}, t_{3}, t_{1} ; y ; 0,0,-y\right)}-1\right\} \\
& +e^{-i\left(t_{1}-t_{2}+t_{3}-t_{4}\right)\left(E_{h}-\varepsilon(0)\right)} e^{s_{X ; r}\left(t_{1}, t_{2}, 0\right)} e^{s_{X ; r}^{*}\left(t_{2}, t_{1}, 0\right)} e^{s_{X ; r}\left(t_{3}, t_{4}, 0\right)} e^{s_{X ; r}^{*}\left(t_{4}, t_{3}, 0\right)} \\
& \quad \times \int_{-\infty}^{\infty} d y e^{S_{X ; r r}\left(t_{1}, t_{3}, t_{2}, t_{4} ; y ; 0,0, y\right)} e^{S_{X ; r r}^{*}\left(t_{4}, t_{2}, t_{3}, t_{1} ; y ; 0,0,-y\right)} \\
& \quad \times(2 \pi i)^{-2}\left(v_{f}\left(t_{1}-t_{4}\right)-y-i 2 \Lambda\right)^{-1}\left(v_{f}\left(t_{2}-t_{3}\right)-y-i 2 \Lambda\right)^{-1}
\end{aligned}
$$

with the number of integrations being reduced to one integration with respect to the relative hole distance $y$. Since the electron-hole interaction affects the form of the integrands the analysis of the result is more complicated than in the case of the twopoint response functions. If the same approximation is applied to the other excitonic 
four-point response functions from Section C.1, one obtains the following identities

$$
\begin{aligned}
& \left\langle\left\langle\tilde{P}_{r}\left(t_{1}\right) \tilde{P}_{r}^{\dagger}\left(t_{2}\right) \tilde{P}_{l}\left(t_{3}\right) \tilde{P}_{l}^{\dagger}\left(t_{4}\right)\right\rangle\right\rangle_{c} \\
& \approx e^{-i\left(t_{1}-t_{2}+t_{3}-t_{4}\right)\left(E_{h}-\varepsilon(0)\right)} e^{s_{X ; r}\left(t_{1}, t_{2}, 0\right)} e^{s_{X ; l}^{*}\left(t_{4}, t_{3}, 0\right)} e^{s_{X ; l}\left(t_{3}, t_{4}, 0\right)} e^{s_{X ; r}^{*}\left(t_{2}, t_{1}, 0\right)} \\
& \quad \times(2 \pi i)^{-2}\left(v_{f}\left(t_{1}-t_{2}\right)-i 2 \Lambda\right)^{-1}\left(v_{f}\left(t_{3}-t_{4}\right)-i 2 \Lambda\right)^{-1} \\
& \quad \times \int_{-\infty}^{\infty} d y\left\{e^{S_{X ; r l}\left(t_{1}, t_{3}, t_{2}, t_{4} ; y ; 0,0, y\right)} e^{S_{X ; l r}^{*}\left(t_{4}, t_{2}, t_{3}, t_{1} ; y ; 0,0,-y\right)}-1\right\}
\end{aligned}
$$

and

$$
\begin{aligned}
& \left\langle\left\langle\tilde{P}_{r}\left(t_{1}\right) \tilde{P}_{l}^{\dagger}\left(t_{2}\right) \tilde{P}_{l}\left(t_{3}\right) \tilde{P}_{r}^{\dagger}\left(t_{4}\right)\right\rangle\right\rangle_{c} \\
& \approx e^{-i\left(t_{1}-t_{2}+t_{3}-t_{4}\right)\left(E_{h}-\varepsilon(0)\right)} e^{s_{X ; r}\left(t_{1}, t_{2}, 0\right)} e^{s_{X ; r}^{*}\left(t_{4}, t_{3}, 0\right)} e^{s_{X ; l}\left(t_{3}, t_{4}, 0\right)} e^{s_{X ; l}^{*}\left(t_{2}, t_{1}, 0\right)} \\
& \quad \times \int_{-\infty}^{\infty} d y e^{i 2 k_{f} y} e^{S_{X ; r l}\left(t_{1}, t_{3}, t_{2}, t_{4} ; y ; 0,0, y\right)} e^{S_{X ; r l}^{*}\left(t_{4}, t_{2}, t_{3}, t_{1} ; y ; 0,0,-y\right)} \\
& \quad \times(2 \pi i)^{-2}\left(v_{f}\left(t_{1}-t_{4}\right)-y-i 2 \Lambda\right)^{-1}\left(v_{f}\left(t_{2}-t_{3}\right)+y-i 2 \Lambda\right)^{-1} .
\end{aligned}
$$

The approximated version of the biexcitonic four-point function in Equation C.27 satisfies the relation

$$
\begin{aligned}
&\left\langle\left\langle\tilde{P}_{r}\left(t_{1}\right) \tilde{P}_{r}\left(t_{2}\right) \tilde{P}_{r}^{\dagger}\left(t_{3}\right) \tilde{P}_{r}^{\dagger}\left(t_{4}\right)\right\rangle\right\rangle_{c} \\
& \approx e^{-i\left(t_{1}+t_{2}-t_{3}-t_{4}\right)\left(E_{h}-\varepsilon(0)\right)} e^{s_{X ; r}\left(t_{1}, t_{4}, 0\right)} e^{s_{X ; r}^{*}\left(t_{4}, t_{1}, 0\right)} e^{s_{X ; r}\left(t_{2}, t_{3}, 0\right)} e^{s_{X ; r}^{*}\left(t_{3}, t_{2}, 0\right)} \\
& \times(2 \pi i)^{-2}\left(v_{f}\left(t_{1}-t_{4}\right)-i 2 \Lambda\right)^{-1}\left(v_{f}\left(t_{2}-t_{3}\right)-i 2 \Lambda\right)^{-1} \\
& \times \int_{-\infty}^{\infty} d y\left\{e^{i\left(t_{2}-t_{3}\right) 2 \varepsilon(y)} e^{S_{X X ; r r}\left(t_{1}, t_{2}, t_{4}, t_{3} ; y ; 0,0, y\right)} e^{S_{X X ; r r}^{*}\left(t_{4}, t_{3}, t_{1}, t_{2} ; y ; 0,0, y\right)}-1\right\} \\
&+ e^{-i\left(t_{1}+t_{2}-t_{3}-t_{4}\right)\left(E_{h}-\varepsilon(0)\right)} e^{s_{X ; r}\left(t_{1}, t_{3}, 0\right)} e^{s_{X ; r}^{*}\left(t_{3}, t_{1}, 0\right)} e^{s_{X ; r}\left(t_{2}, t_{4}, 0\right)} e^{s_{X ; r}^{*}\left(t_{4}, t_{2}, 0\right)} \\
& \times(2 \pi i)^{-2}\left(v_{f}\left(t_{1}-t_{3}\right)-i 2 \Lambda\right)^{-1}\left(v_{f}\left(t_{2}-t_{4}\right)-i 2 \Lambda\right)^{-1} \\
& \times \int_{-\infty}^{\infty} d y\left\{e^{i\left(t_{2}-t_{3}\right) 2 \varepsilon(y)} e^{S_{X X ; r r}\left(t_{1}, t_{2}, t_{3}, t_{4} ; y ; 0,0, y\right)} e^{S_{X X ; r r}^{*}\left(t_{4}, t_{3}, t_{2}, t_{1} ; y ; 0,0,-y\right)}-1\right\} \\
&- e^{-i\left(t_{1}+t_{2}-t_{3}-t_{4}\right)\left(E_{h}-\varepsilon(0)\right)} e^{s_{X ; r}\left(t_{1}, t_{4}, 0\right)} e^{s_{X ; r}^{*}\left(t_{4}, t_{1}, 0\right)} e^{s_{X ; r}\left(t_{2}, t_{3}, 0\right)} e^{s_{X ; r}^{*}\left(t_{3}, t_{2}, 0\right)} \\
& \times \int_{-\infty}^{\infty} d y e^{i\left(t_{2}-t_{3}\right) 2 \varepsilon(y)} e^{S_{X X ; r r}\left(t_{1}, t_{2}, t_{4}, t_{3} ; y ; 0,0, y\right)} e^{S_{X X ; r r}^{*}\left(t_{4}, t_{3}, t_{1}, t_{2} ; y ; 0,0, y\right)} \\
& \times(2 \pi i)^{-2}\left(v_{f}\left(t_{1}-t_{3}\right)-y-i 2 \Lambda\right)^{-1}\left(v_{f}\left(t_{2}-t_{4}\right)+y-i 2 \Lambda\right)^{-1} \\
&- e^{-i\left(t_{1}+t_{2}-t_{3}-t_{4}\right)\left(E_{h}-\varepsilon(0)\right)} e^{s_{X ; r}\left(t_{1}, t_{3}, 0\right)} e^{s_{X ; r}^{*}\left(t_{3}, t_{1}, 0\right)} e^{s_{X ; r}\left(t_{2}, t_{4}, 0\right)} e^{s_{X ; r}^{*}\left(t_{4}, t_{2}, 0\right)} \\
& \times \int_{-\infty}^{\infty} d y e^{i\left(t_{2}-t_{3}\right) 2 \varepsilon(y)} e^{S_{X X ; r r}\left(t_{1}, t_{2}, t_{3}, t_{4} ; y ; 0,0, y\right)} e^{S_{X X ; r r}^{*}\left(t_{4}, t_{3}, t_{2}, t_{1} ; y ; 0,0,-y\right)} \\
& \times(2 \pi i)^{-2}\left(v_{f}\left(t_{1}-t_{4}\right)-y-i 2 \Lambda\right)^{-1}\left(v_{f}\left(t_{2}-t_{3}\right)+y-i 2 \Lambda\right)^{-1}
\end{aligned}
$$

whereas the biexcitonic four-point functions from Equations C.32 and C.33 are ap- 
proximated by the following expressions

$$
\begin{aligned}
& \left\langle\left\langle\tilde{P}_{r}\left(t_{1}\right) \tilde{P}_{l}\left(t_{2}\right) \tilde{P}_{r}^{\dagger}\left(t_{3}\right) \tilde{P}_{l}^{\dagger}\left(t_{4}\right)\right\rangle\right\rangle_{c} \\
& \approx e^{-i\left(t_{1}+t_{2}-t_{3}-t_{4}\right)\left(E_{h}-\varepsilon(0)\right)} e^{s_{X ; r}\left(t_{1}, t_{3}, 0\right)} e^{s_{X ; r}^{*}\left(t_{3}, t_{1}, 0\right)} e^{s_{X ; l}\left(t_{2}, t_{4}, 0\right)} e^{s_{X ; l}^{*}\left(t_{4}, t_{2}, 0\right)} \\
& \quad \times(2 \pi i)^{-2}\left(v_{f}\left(t_{1}-t_{3}\right)-i 2 \Lambda\right)^{-1}\left(v_{f}\left(t_{2}-t_{4}\right)-i 2 \Lambda\right)^{-1} \\
& \quad \times \int_{-\infty}^{\infty} d y\left\{e^{i\left(t_{2}-t_{3}\right) 2 \varepsilon(y)} e^{S_{X X ; r l}\left(t_{1}, t_{2}, t_{3}, t_{4} ; y ; 0,0, y\right)} e^{S_{X X ; l r}^{*}\left(t_{4}, t_{3}, t_{2}, t_{1} ; y ; 0,0,-y\right)}-1\right\} \\
& -e^{-i\left(t_{1}+t_{2}-t_{3}-t_{4}\right)\left(E_{h}-\varepsilon(0)\right)} e^{s_{X ; r}\left(t_{1}, t_{4}, 0\right)} e^{s_{X ; r}^{*}\left(t_{3}, t_{2}, 0\right)} e^{s_{X ; l}\left(t_{2}, t_{3}, 0\right)} e^{s_{X ; l}^{*}\left(t_{4}, t_{1}, 0\right)} \\
& \quad \times \int_{-\infty}^{\infty} d y e^{i 2 k_{f} y} e^{i\left(t_{2}-t_{3}\right) 2 \varepsilon(y)} e^{S_{X X ; r l}\left(t_{1}, t_{2}, t_{4}, t_{3} ; y ; 0,0, y\right)} e^{S_{X X ; l r}^{*}\left(t_{4}, t_{3}, t_{1}, t_{2} ; y ; 0,0, y\right)} \\
& \quad \times(2 \pi i)^{-2}\left(v_{f}\left(t_{1}-t_{3}\right)-y-i 2 \Lambda\right)^{-1}\left(v_{f}\left(t_{2}-t_{4}\right)-y-i 2 \Lambda\right)^{-1}
\end{aligned}
$$

and

$$
\begin{aligned}
& \left\langle\left\langle\tilde{P}_{r}\left(t_{1}\right) \tilde{P}_{l}\left(t_{2}\right) \tilde{P}_{l}^{\dagger}\left(t_{3}\right) \tilde{P}_{r}^{\dagger}\left(t_{4}\right)\right\rangle\right\rangle_{c} \\
& \approx e^{-i\left(t_{1}+t_{2}-t_{3}-t_{4}\right)\left(E_{h}-\varepsilon(0)\right)} e^{s_{X ; r}\left(t_{1}, t_{4}, 0\right)} e^{s_{X ; r}^{*}\left(t_{4}, t_{1}, 0\right)} e^{s_{X ; l}\left(t_{2}, t_{3}, 0\right)} e^{s_{X ; l}^{*}\left(t_{3}, t_{2}, 0\right)} \\
& \quad \times(2 \pi i)^{-2}\left(v_{f}\left(t_{1}-t_{4}\right)-i 2 \Lambda\right)^{-1}\left(v_{f}\left(t_{2}-t_{3}\right)-i 2 \Lambda\right)^{-1} \\
& \quad \times \int_{-\infty}^{\infty} d y\left\{e^{i\left(t_{2}-t_{3}\right) 2 \varepsilon(y)} e^{S_{X X ; r l}\left(t_{1}, t_{2}, t_{4}, t_{3} ; y ; 0,0, y\right)} e^{S_{X X ; r l}^{*}\left(t_{4}, t_{3}, t_{1}, t_{2} ; y ; 0,0, y\right)}-1\right\} \\
& -e^{-i\left(t_{1}+t_{2}-t_{3}-t_{4}\right)\left(E_{h}-\varepsilon(0)\right)} e^{s_{X ; r}\left(t_{1}, t_{3}, 0\right)} e^{s_{X ; r}^{*}\left(t_{4}, t_{2}, 0\right)} e^{s_{X ; l}\left(t_{2}, t_{4}, 0\right)} e^{s_{X ; l}^{*}\left(t_{3}, t_{1}, 0\right)} \\
& \quad \times \int_{-\infty}^{\infty} d y e^{i 2 k_{f} y} e^{i\left(t_{2}-t_{3}\right) 2 \varepsilon(y)} e^{S_{X X ; r l}\left(t_{1}, t_{2}, t_{3}, t_{4} ; y ; 0,0, y\right)} e^{S_{X X ; r l}^{*}\left(t_{4}, t_{3}, t_{2}, t_{1} ; y ; 0,0,-y\right)} \\
& \quad \times(2 \pi i)^{-2}\left(v_{f}\left(t_{1}-t_{4}\right)-y-i 2 \Lambda\right)^{-1}\left(v_{f}\left(t_{2}-t_{3}\right)-y-i 2 \Lambda\right)^{-1} .
\end{aligned}
$$

Some of these functions can be simplified further since the width of the function $d$ is assumed to be large in comparison with the inverse Fermi vector $\left(\Lambda \gg k_{f}^{-1}\right)$. It is then possible to neglect the response function in Equation C.40 as well as the second contributions in Equations C.42 and C.43 because of the fast oscillating factor $e^{i 2 k_{f} y}$ which appears in the respective integrals.

Unfortunately, the integrals in the approximated expressions for the four-point response functions are still too complicated to allow a simple description of their dynamics. Nevertheless, it is possible to estimate the leading time behavior for the four-wave mixing signals from Equations 3.70 and 3.71, if the external time $t$ is large compared with the delay time $T$. To this end, the parts of the integrands in Equations C.38 to C.43 which are affected by the presence of the interaction potential have to be subjected to a closer analysis.

The contributions of the excitonic response functions to the four-wave-mixing signal are obtained, if the time variables $t_{2}$ and $t_{4}$ in Equations C.38 and C.39 are set equal to zero while the values for the other two time variables are determined by the equations $t_{1}=-T$ and $t_{3}=t$. In the case of the excitonic response function of Equation 


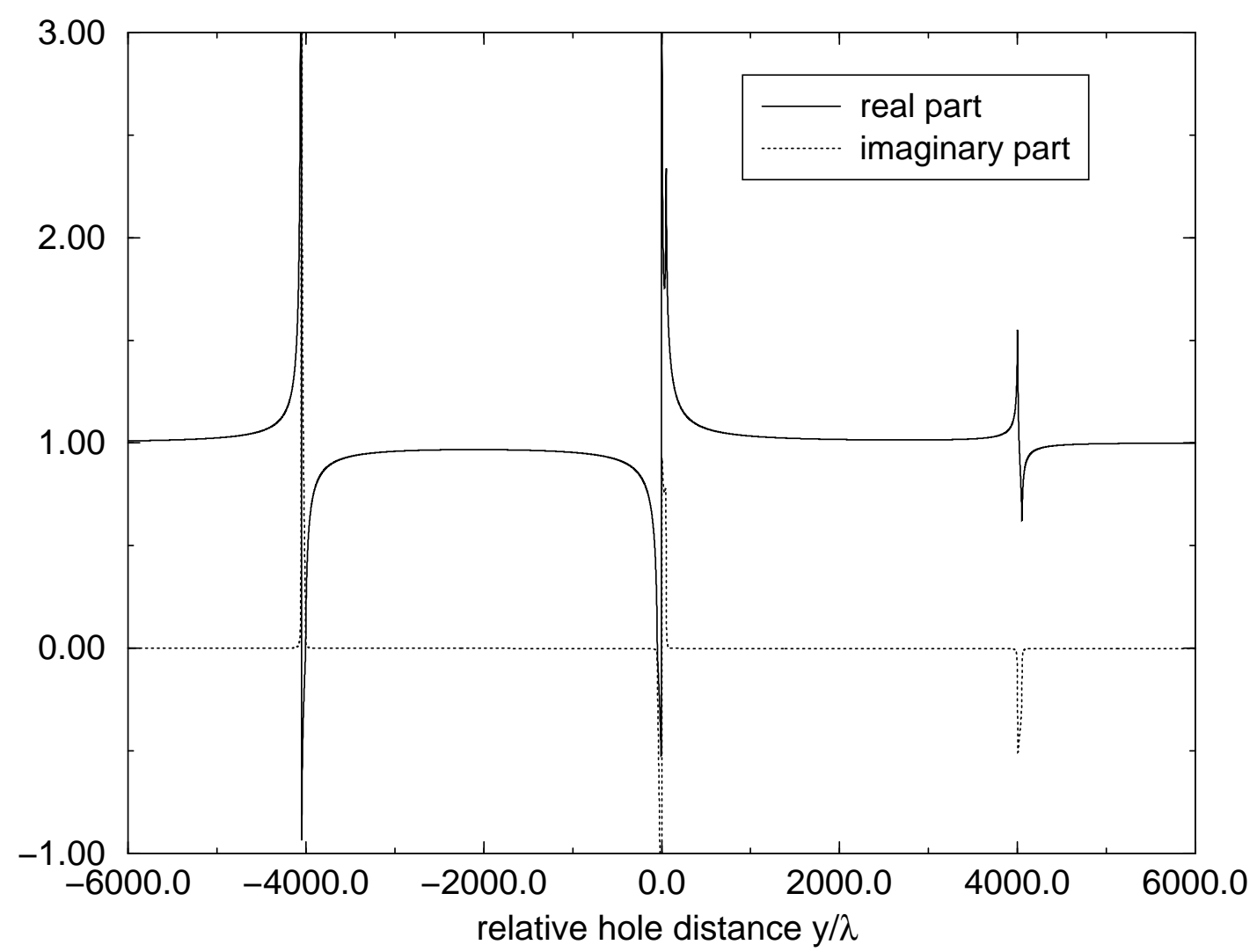

Figure C.1: The real and the imaginary part of the function from Equation C.44 for the parameters $v_{f} t=4000 \lambda, v_{f} T=50 \lambda$ and $\frac{\tilde{v}_{0}}{2 \pi v_{f}}=-0.375$

C.38 this procedure yields the following term

$$
\begin{aligned}
& e^{S_{X ; r r}(-T, t, 0,0 ; y ; 0,0, y)} e^{S_{X ; r r}^{*}(0,0, t,-T ; y ; 0,0,-y)} \\
& =e^{H^{*}\left(-v_{f} T+y, y\right)} e^{H^{*}\left(-v_{f} T-y,-y\right)} e^{H^{*}\left(-v_{f} t+y,-v_{f}(t+T)+y\right)} e^{H^{*}\left(-v_{f} t-y,-v_{f}(t+T)-y\right)} \\
& \quad \times e^{G^{*}\left(-v_{f} T-y,-y\right)} e^{G^{*}\left(-v_{f} T-y,-y\right)} e^{G^{*}\left(-v_{f} t-y,-v_{f}(t+T)-y\right)} e^{G^{*}\left(-v_{f} t-y,-v_{f}(t+T)-y\right)}
\end{aligned}
$$

in the two integrands. The corresponding expression for the response function in Equation C.39 is given by

$$
\begin{aligned}
e^{S_{X ; r l}(-T, t, 0,0 ; y ; 0,0, y)} e^{S_{X ; l r}^{*}(0,0, t,-T ; y ; 0,0,-y)} & e^{H^{*}\left(-v_{f} T+y, y\right)} e^{H^{*}\left(-v_{f} T-y,-y\right)} e^{H^{*}\left(-v_{f} t+y,-v_{f}(t+T)+y\right)} e^{H^{*}\left(-v_{f} t-y,-v_{f}(t+T)-y\right)} \\
& \times e^{G^{*}\left(-v_{f} T+y, y\right)} e^{G^{*}\left(-v_{f} T-y,-y\right)} e^{G^{*}\left(-v_{f} t+y,-v_{f}(t+T)+y\right)} e^{G^{*}\left(-v_{f} t-y,-v_{f}(t+T)-y\right)} .
\end{aligned}
$$

Both functions show a similar structure and are approximately described with the help of the formulae D.30 and D.31 in Appendix D. It turns out that the expressions only 
differ from unity in three regions near $y=0, y=-v_{f} t$ and $y=v_{f} t$ and that the width of these regions is determined by the delay time $T$. In order to illustrate this statement, the function on the right-hand side of Equation C.44 is depicted in Figure C.1 where it is assumed that the Fourier transform of the interaction potential is given by $\tilde{v}(q)=\tilde{v}_{0} e^{-\lambda|q|}$.

The biexcitonic contributions to the four-wave-mixing signal are calculated with the help of the response functions in Equations C. 41 to C. 43 where the time variables $t_{3}$ and $t_{4}$ are set equal to zero while the values $t$ and $-T$ are assigned to the first two time variables.

If $t_{1}=t$ and $t_{2}=-T$, the integrands which appear in Equation C.41 contain the functions

$$
\begin{aligned}
e^{S_{X X ; r r}(t,-T, 0,0 ; y ; 0,0, y)} e^{S_{X X ; r r}^{*}(0,0, t,-T ; y ; 0,0, y)} e^{-i T 2 \varepsilon(y)} \\
=e^{S_{X X ; r r}(t,-T, 0,0 ; y ; 0,0, y)} e_{X X ; r r}^{S_{X}^{*}(0,0,-T, t ; y ; 0,0,-y)} e^{-i T 2 \varepsilon(y)} \\
=e^{H^{*}\left(-v_{f} T+y, y\right)} e^{H^{*}\left(-v_{f} T-y,-y\right)} e^{H^{*}\left(v_{f} t+y, v_{f}(t+T)+y\right)} e^{H^{*}\left(v_{f} t-y, v_{f}(t+T)-y\right)} \\
\quad \times e^{G^{*}\left(-v_{f} T+y, y\right)} e^{G^{*}\left(-v_{f} T+y, y\right)} e^{G^{*}\left(v_{f} t-y, v_{f}(t+T)-y\right)} e^{G^{*}\left(v_{f} t-y, v_{f}(t+T)-y\right)} e^{-i T 2 \varepsilon(y)}
\end{aligned}
$$

whereas the corresponding terms in Equations C. 42 and C.43 are determined by the relations

$$
\begin{aligned}
e^{S_{X X} ; r l}(t,-T, 0,0 ; y ; 0,0, y) & e^{S_{X X}^{*} ; l_{r}(0,0,-T, t ; ; ; 0,0,-y)} e^{-i T 2 \varepsilon(y)} \\
= & e^{S_{X X ; r l}(t,-T, 0,0 ; y ; 0,0, y)} e^{S_{X X ; r l}^{*}(0,0, t,-T ; y ; 0,0, y)} e^{-i T 2 \varepsilon(y)} \\
= & e^{H^{*}\left(-v_{f} T+y, y\right)} e^{H^{*}\left(-v_{f} T-y,-y\right)} e^{H^{*}\left(v_{f} t+y, v_{f}(t+T)+y\right)} e^{H^{*}\left(v_{f} t-y, v_{f}(t+T)-y\right)} \\
& \times e^{G^{*}\left(-v_{f} T+y, y\right)} e^{G^{*}\left(-v_{f} T-y,-y\right)} e^{G^{*}\left(v_{f} t+y, v_{f}(t+T)+y\right)} e^{G^{*}\left(v_{f} t-y, v_{f}(t+T)-y\right)} e^{-i T 2 \varepsilon(y) .}
\end{aligned}
$$

A comparison shows that the expressions in Equations C.46 and C.47 are similar to those in Equations C.44 and C.45. The sole qualitative difference is the factor $e^{-i T 2 \varepsilon(y)}$ which only influences the form of the functions near $y=0$ since $\varepsilon(y)$ decays like $y^{-2}$ for large absolute values of $y$.

If $t_{1}=-T$ and $t_{2}=t$, the integrands of the biexcitonic response function in Equation C.41 contain the following terms

$$
\begin{aligned}
e^{S_{X X ; r r}(-T, t, 0,0 ; y ; 0,0, y)} e^{S_{X X ; r r}^{*}(0,0,-T, t ; y ; 0,0, y)} e^{i t 2 \varepsilon(y)} \\
=e^{S_{X X ; r r}(-T, t, 0,0 ; ; y ; 0,0, y)} e_{X X ; r r}^{S_{X}^{*}(0,0, t,-T ; y ; 0,0,-y)} e^{i t 2 \varepsilon(y)} \\
=e^{H^{*}\left(-v_{f} T+y, y\right)} e^{H^{*}\left(-v_{f} T-y,-y\right)} e^{H^{*}\left(v_{f} t+y,-v_{f}(t+T)-y\right)} e^{H^{*}\left(v_{f} t-y,-v_{f}(t+T)+y\right)} \\
\quad \times e^{G^{*}\left(-v_{f} T-y, y\right)} e^{G^{*}\left(-v_{f} T-y, y\right)} e^{G^{*}\left(v_{f} t+y,-v_{f}(t+T)-y\right)} e^{G^{*}\left(v_{f} t+y,-v_{f}(t+T)-y\right)} e^{i t 2 \varepsilon(y)}
\end{aligned}
$$

while the corresponding expressions for the biexcitonic functions in Equations C.42 
and C.43 are given by

$$
\begin{aligned}
& e^{S_{X X ; r l}(-T, t, 0,0 ; y ; 0,0, y)} e^{S_{X X ; l r}^{*}(0,0, t,-T ; y ; 0,0,-y)} e^{i t 2 \varepsilon(y)} \\
&=e^{S_{X X ; r l}(-T, t, 0,0 ; y ; 0,0, y)} e^{S_{X X ; r l}^{*}(0,0,-T, t ; y ; 0,0, y)} e^{i t 2 \varepsilon(y)} \\
&=e^{H^{*}\left(-v_{f} T+y, y\right)} e^{H^{*}\left(-v_{f} T-y,-y\right)} e^{H^{*}\left(v_{f} t+y,-v_{f}(t+T)-y\right)} e^{H^{*}\left(v_{f} t-y,-v_{f}(t+T)+y\right)} \\
& \times e^{G^{*}\left(-v_{f} T+y, y\right)} e^{G^{*}\left(-v_{f} T-y,-y\right)} e^{G^{*}\left(v_{f} t+y,-v_{f}(t+T)-y\right)} e^{G^{*}\left(v_{f} t-y,-v_{f}(t+T)+y\right)} e^{i t 2 \varepsilon(y)} .
\end{aligned}
$$

It turns out that the structure of these functions differs considerably from the structure of the functions in Equations C.44 to C.47. The absolute values of the expressions on the right-hand sides of Equations C.48 and C.49 are still approximately equal to unity unless $y$ is located in the vicinities of the points $y=0, y=v_{f} t$ and $y=-v_{f} t$. In the regions between these points, however, the phases of the functions approximately assume constant values which are not necessarily equal to zero. This can be put down to the fact that several of the exponents of the exponential functions on the right-hand sides of Equations C.48 and C.49 are similar to the functions in D.32 and D.33. The behavior of the total functions can be described by the two equations

$$
\begin{aligned}
& e^{S_{X X ; r r}(-T, t, 0,0 ; y ; 0,0, y)} e^{S_{X X ; r r}^{*}(0,0,-T, t ; y ; 0,0, y)} e^{i t 2 \varepsilon(y)} \\
& =e^{S_{X X ; r r}(-T, t, 0,0 ; y ; 0,0, y)} e^{S_{X X ; r r}^{*}(0,0, t,-T ; y ; 0,0,-y)} e^{i t 2 \varepsilon(y)} \\
& \approx\left\{\theta\left(y-v_{f} t\right)+\theta\left(-y-v_{f} t\right)\right\}+e^{-2 \pi i\left\{2 \frac{\tilde{v}(0)}{2 \pi v_{f}}+\frac{\tilde{v}^{2}(0)}{\left(2 \pi v_{f}\right)^{2}}\right\}}\left\{\theta\left(y+v_{f} t\right)-\theta(y)\right\} \\
& +e^{-2 \pi i \frac{\tilde{v}^{2}(0)}{\left(2 \pi v_{f}\right)^{2}}}\left\{\theta(y)-\theta\left(y-v_{f} t\right)\right\}
\end{aligned}
$$

and

$$
\begin{aligned}
& e^{S_{X X ; r l}(-T, t, 0,0 ; y ; 0,0, y)} e^{S_{X X ; l r}^{*}(0,0, t,-T ; y ; 0,0,-y)} e^{i t 2 \varepsilon(y)} \\
& =e^{S_{X X ; r l}(-T, t, 0,0 ; y ; 0,0, y)} e^{S_{X X ; r l}^{*}(0,0,-T, t ; y ; 0,0, y)} e^{i t 2 \varepsilon(y)} \\
& \approx\left\{\theta\left(y-v_{f} t\right)+\theta\left(-y-v_{f} t\right)\right\}+e^{-2 \pi i\left\{\frac{\tilde{v}(0)}{2 \pi v_{f}}+\frac{\tilde{v}^{2}(0)}{\left(2 \pi v_{f}\right)^{2}}\right\}}\left\{\theta\left(y+v_{f} t\right)-\theta\left(y-v_{f} t\right)\right\}
\end{aligned}
$$

outside of the vicinities of the three points mentioned above, if the time $t$ is considerably larger than the delay time $T$. It is justified to neglect the term $e^{i t 2 \varepsilon(y)}$ since it only affects a decreasing fraction of the interval $\left[-v_{f} t, v_{f} t\right]$, if $t$ increases. This is due to the quadratic decay of $\varepsilon(y)$ for large values of $|y|$.

In Figures C. 2 and C. 3 the real and imaginary part of the expression on the righthand side of Equation C. 48 have been plotted together with the corresponding approximated curves where the interaction potential and all other parameters are the same as in Figure C.1. A comparison shows that there exists a good agreement between the exact functions and the approximated ones apart from the three regions where a phase 


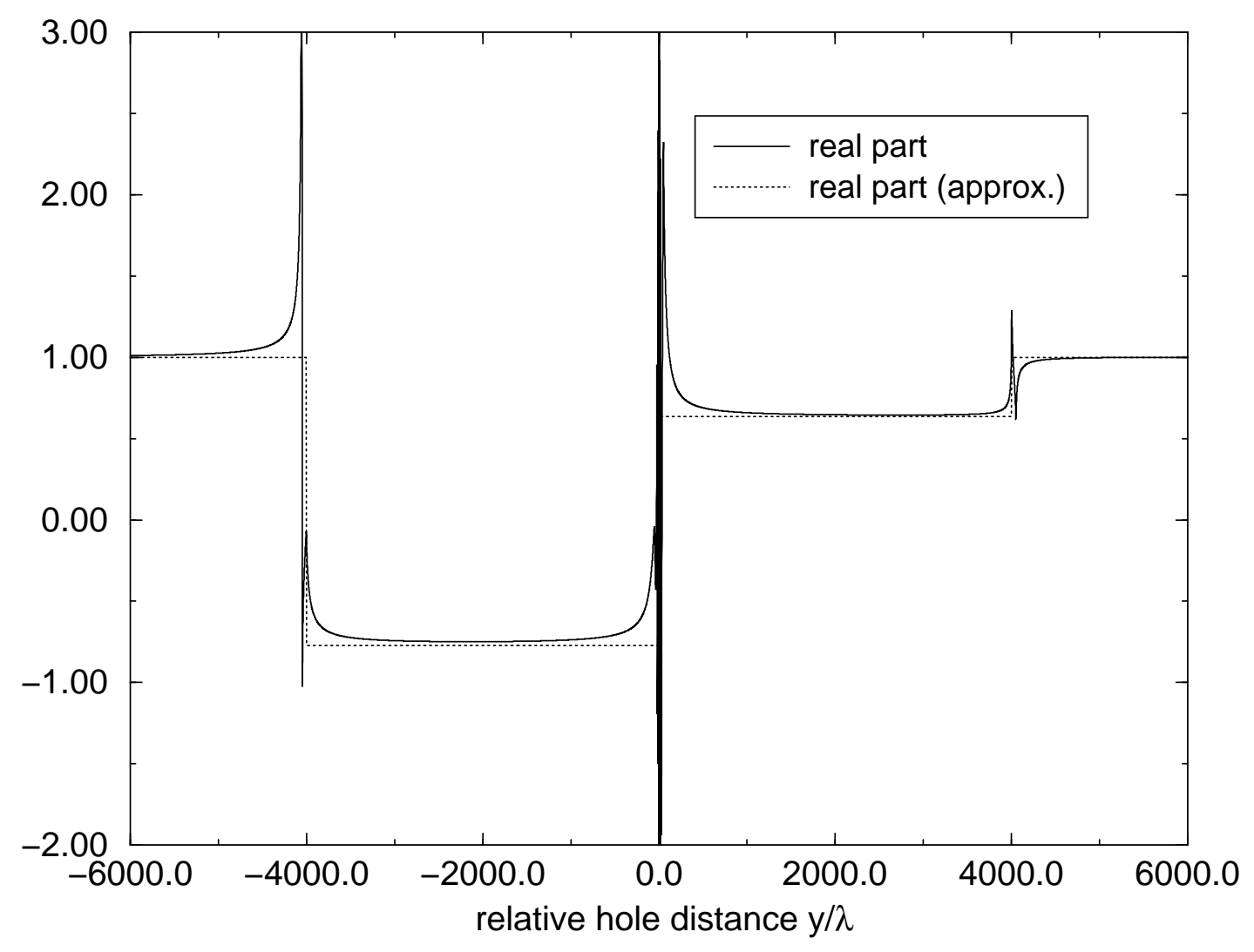

Figure C.2: The real part of the function from Equation C.48 and the corresponding approximated curve for the parameters $v_{f} t=4000 \lambda, v_{f} T=50 \lambda$ and $\frac{\tilde{v}_{0}}{2 \pi v_{f}}=-0.375$

shift occurs. Near the origin the exact functions exhibit fast oscillations which are caused by the factor $e^{i t 2 \varepsilon(y)}$.

If the four-wave mixing signal is calculated with the help of the approximated response functions, the functions from Equations C.44 to C.49 appear in the integrands of two different types of integrals.

Integrals of the first type can be found in the second contribution to the excitonic response function in Equation C.38 and in the third and the fourth contribution to the biexcitonic response function in Equation C.41. All these integrals contain two linear fractions as factors in their integrands which lead to two sharp peaks at $y=v_{f} t$ and $y=v_{f} T$ or $y=-v_{f} t$ and $y=-v_{f} T$. Because of these fractions the decay of the integrands is proportional to the inverse square of $y$ for large positive or negative values of $y$. If $t$ goes to infinity, the integrals converge towards zero like $t^{-1}$ while the complete contributions are proportional to $t^{-(1+\alpha)}$ because of the factors in front of the integrals.

Integrals of the second type appear in the first contribution to the excitonic response function in Equation C. 38 and in the first two contributions to the biexcitonic response 


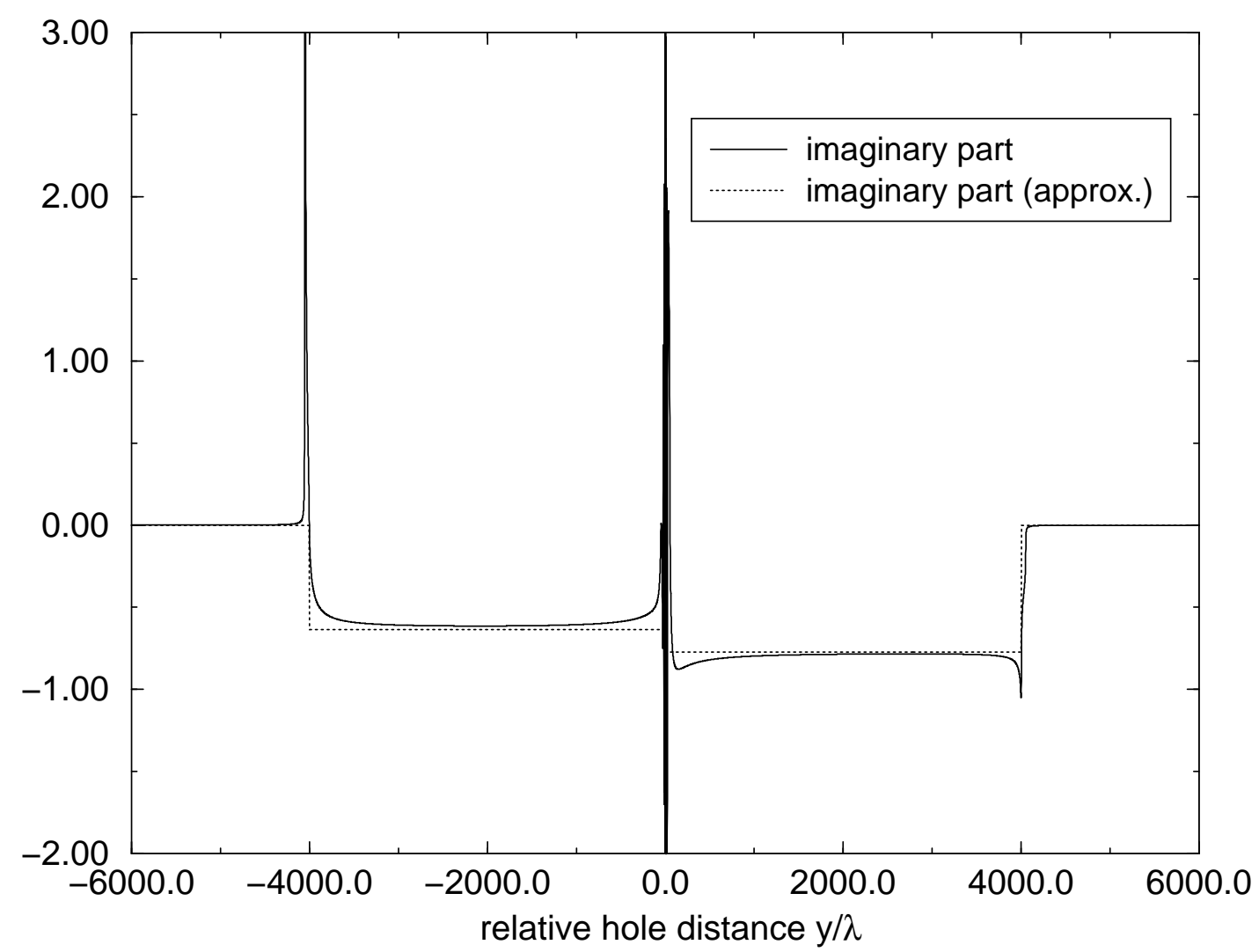

Figure C.3: The imaginary part of the function from Equation C.48 and the corresponding approximated curve for the parameters $v_{f} t=4000 \lambda, v_{f} T=50 \lambda$ and $\frac{\tilde{v}_{0}}{2 \pi v_{f}}=-0.375$

function in Equation C.41. In addition to that, they can be found in the approximated expressions for the four-point response functions in Equations C.39, C.42 and C.43. Their integrands are given by the difference between one of the ten functions from Equations C.44 to C. 49 and unity.

If the integrals of the different functions are compared, it emerges that there exists a significant difference between the dynamics of the integrals which contain one of the functions from Equations C.44 to C. 47 and those integrals which contain one of the functions from Equations C.48 or C.49. The integrands for the integrals of the first kind only differ from zero in the vicinities of the points $y=v_{f} t, y=0$ and $y=-v_{f} t$ and the absolute values of these integrals are bounded as functions of $t$. In contrast to that, the integrals which contain one of the functions from Equation C.48 and C.49 show a linear increase for large values of the variable $t$.

This behavior can be explained by the fact that the integrands can approximately be described with the help of the step functions from Equations C.50 and C.51. Consequently, it is justified to approximate the exact integrals by using the following for- 


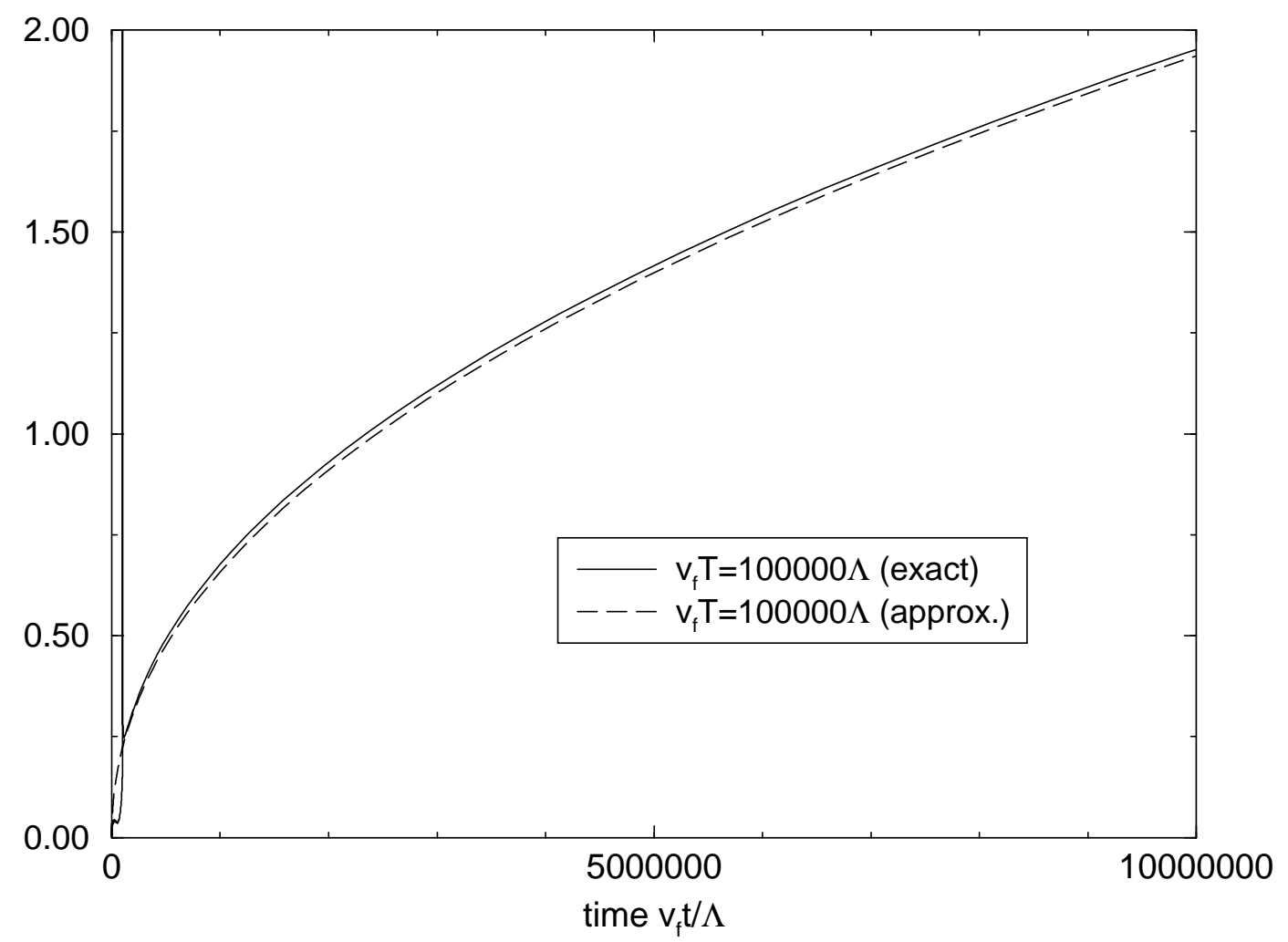

Figure C.4: The absolute value of the four-wave-mixing signal for the delay time $v_{f} T=100000 \Lambda$ and the corresponding approximated signal with the interaction parameters $\frac{\tilde{v}_{0}}{2 \pi v_{f}}=-0.375$ and $\lambda=1000 \Lambda$

mulae

$$
\begin{aligned}
& \int_{-\infty}^{\infty} d y\left\{e^{i t 2 \varepsilon(y)} e^{S_{X X ; r r}(-T, t, 0,0 ; y ; 0,0, y)} e^{S_{X X ; r r}^{*}(0,0,-T, t ; y ; 0,0, y)}-1\right\} \\
& =\int_{-\infty}^{\infty} d y\left\{e^{i t 2 \varepsilon(y)} e^{S_{X X ; r r}(-T, t, 0,0 ; y ; 0,0, y)} e^{S_{X X ; r r}^{*}(0,0, t,-T ; y ; 0,0,-y)}-1\right\} \\
& \approx\left|v_{f} t\right|\left\{e^{-2 \pi i\left\{2 \frac{\tilde{v}(0)}{2 \pi v_{f}}+\frac{\tilde{v}^{2}(0)}{\left(2 \pi v_{f}\right)^{2}}\right\}}+e^{-2 \pi i \frac{\tilde{v}^{2}(0)}{\left(2 \pi v_{f}\right)^{2}}}-2\right\}
\end{aligned}
$$

and

$$
\begin{aligned}
& \int_{-\infty}^{\infty} d y\left\{e^{i t 2 \varepsilon(y)} e^{S_{X X ; r l}(-T, t, 0,0 ; y ; 0,0, y)} e^{S_{X X ; l r}^{*}(0,0, t,-T ; y ; 0,0,-y)}-1\right\} \\
& =\int_{-\infty}^{\infty} d y\left\{e^{i t 2 \varepsilon(y)} e^{S_{X X ; r l}(-T, t, 0,0 ; y ; 0,0, y)} e^{S_{X X ; r l}^{*}(0,0,-T, t ; y ; 0,0, y)}-1\right\} \\
& \approx 2\left|v_{f} t\right|\left\{e^{-2 \pi i\left\{\frac{\tilde{v}(0)}{2 \pi v_{f}}+\frac{\tilde{v}^{2}(0)}{\left(2 \pi v_{f}\right)^{2}}\right\}}-1\right\}
\end{aligned}
$$




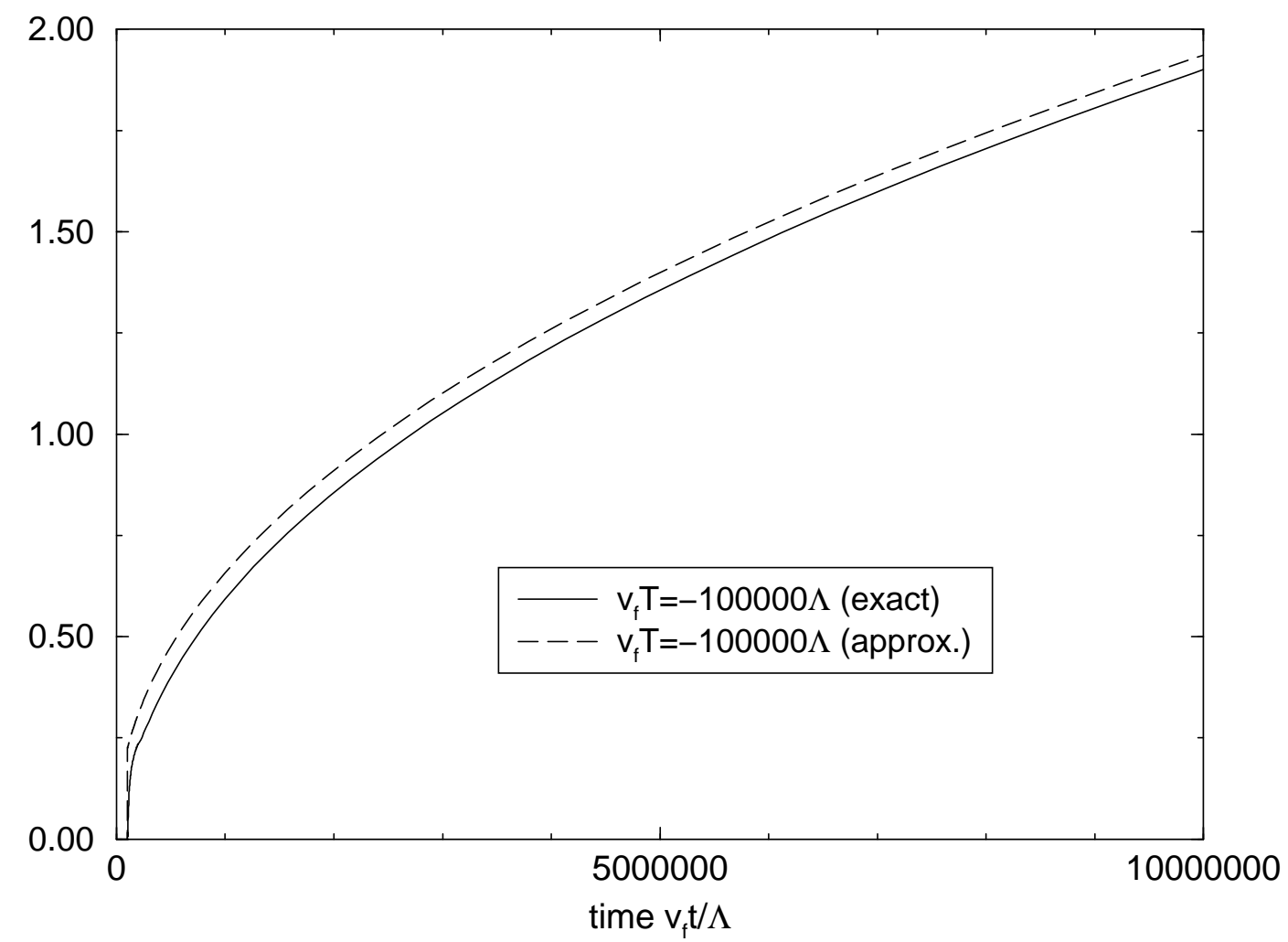

Figure C.5: The absolute value of the four-wave-mixing signal for the delay time $v_{f} T=-100000 \Lambda$ and the corresponding approximated signal with the interaction parameters $\frac{\tilde{v}_{0}}{2 \pi v_{f}}=-0.375$ and $\lambda=1000 \Lambda$

provided that the external time $t$ is considerably larger than the absolute value of the delay time $T$. All integrals of the second type have the same prefactor which is approximately given by

$$
\begin{aligned}
& e^{-i(t-T)\left(E_{h}-\varepsilon(0)\right)} e^{s_{X ; r}(t, 0,0)} e^{s_{X ; r}^{*}(0, t, 0)} e^{s_{X ; r}(-T, 0,0)} e^{s_{X ; r}^{*}(0,-T, 0)} \\
& =e^{-i(t-T)\left(E_{h}-\varepsilon(0)\right)} e^{s_{X ; l}(t, 0,0)} e^{s_{X ; l}^{*}(0, t, 0)} e^{s_{X ; r}(-T, 0,0)} e^{s_{X ; r}^{*}(0,-T, 0)} \\
& \approx e^{-i(t-T)\left(E_{h}-\varepsilon(0)\right)}\left|v_{f} T\right|^{-(1+\alpha)}\left|v_{f} t\right|^{-(1+\alpha)} \frac{\left\{\Theta(-T) z^{*} z^{*}-\Theta(T) z z^{*}\right\}}{(2 \pi i)^{2}},
\end{aligned}
$$

provided that $t \gg|T| \gg x_{\tilde{v}} v_{f}^{-1}$ (confer Appendix D).

If all results of the analysis are combined, it turns out that the dominant contribution to the four-wave-mixing signal for large times $t$ comes from the first two terms of the biexcitonic response function in Equation C.41 and from the first terms of the biexcitonic response functions in Equations C.42 and C.43, if $t_{1}$ is set equal to $-T$ and $t_{2}$ is set equal to $t$ in all functions. Therefore the four-wave mixing signals from 
Equations 3.70 and 3.71 are approximately described by the following relation

$$
\begin{aligned}
\lim _{L \rightarrow \infty} \frac{1}{L} P_{2 \vec{k}_{1}-\vec{k}_{2}}^{(3)}(t) & \approx e^{-i\left(\omega t-\left(2 \vec{k}_{1}-\vec{k}_{2}\right) \vec{R}\right)} e^{-i(t-T)\left(E_{h}-\varepsilon(0)-\omega\right)} e^{-\Gamma_{x}(t+T)} e^{-\Gamma_{x x} t} \\
& \times\left|v_{f} T\right|^{-(1+\alpha)}\left|v_{f} t\right|^{-\alpha} \frac{\left\{\Theta(T) z z^{*}-\Theta(-T) z^{*} z^{*}\right\}}{(2 \pi i)^{2}} \\
& \times \frac{(-i)^{3}}{4}\left\{\left\{e^{-2 \pi i \frac{\tilde{v}(0)}{2 \pi v_{f}}}+1\right\}^{2} e^{-2 \pi i \tilde{v}^{2}(0)}\left(\frac{\left.\tilde{v}_{f}\right)^{2}}{\left(2 \pi v^{2}\right.}-4\right\}\right.
\end{aligned}
$$

if $t \gg T$ and $T \gg x_{\tilde{v}} v_{f}^{-1}$ and $\Gamma_{x}=\Gamma_{x x}=\Gamma_{0}=0$. In Figures C.4 and C.5 the absolute values of the four-wave-mixing signals for a positive and a negative delay time, which are calculated with the help of Equations C.38 to C.43, are plotted together with the corresponding approximated curves where the interaction function and all system parameters are the same as those which have been used for the calculation of the other figures in this section. As expected, a comparison of the different functions shows that the expression on the right-hand side of Equation C.55 describes the dominant behavior of the signals well, if $t$ is considerably larger than the absolute value of the delay time. However, if $t$ and $T$ are of the same order of magnitude, the signals differ significantly from the approximated curves. One of the most important features of the four-wave-mixing signals which is not reproduced by the approximated functions is the sharp peak which appears at $t=T$, if the delay time is positive. This peak is due to the third contribution and the fourth contribution to the response function in Equation C.41 which have been neglected when deriving Equation C.55. 


\section{Appendix D}

\section{Operator Relations}

According to the results of Section 3.4 and Appendix C the time behavior of the response functions is determined by the free dynamics of the auxiliary operators which have been defined in Section 3.2 when introducing the bosonic representation. In the case of the bosonic and fermionic operators which are used for the representation of the field operators $\psi_{e r}(x)$ and $\psi_{e l}(x)$ in Equations 3.27 and 3.28 the free time behavior is described by the equations

$$
\begin{aligned}
& \varphi_{r ; 0}(x, t)=\varphi_{r}\left(x-v_{f} t\right)=\lim _{\alpha \searrow 0}-i \sum_{q>0} \sqrt{\frac{2 \pi}{L q}} e^{-\alpha q} e^{i q\left(x-v_{f} t\right)} b_{q r}, \\
& \varphi_{l ; 0}(x, t)=\varphi_{l}\left(x+v_{f} t\right)=\lim _{\alpha \searrow 0}-i \sum_{q>0} \sqrt{\frac{2 \pi}{L q}} e^{-\alpha q} e^{-i q\left(x+v_{f} t\right)} b_{q l}
\end{aligned}
$$

and

$$
\begin{aligned}
& U_{r ; 0}(t)=e^{-i v_{f} t \frac{\pi}{L}\left\{2 N_{r}+1\right\}} U_{r}=e^{-i v_{f} t \frac{\pi}{L} N_{r}} U_{r} e^{-i v_{f} t \frac{\pi}{L} N_{r}}, \\
& U_{l ; 0}(t)=e^{-i v_{f} t \frac{\pi}{L}\left\{2 N_{l}+1\right\}} U_{l}=e^{-i v_{f} t \frac{\pi}{L} N_{l}} U_{l} e^{-i v_{f} t \frac{\pi}{L} N_{l}} .
\end{aligned}
$$

The index 0 denotes that the operators are calculated in the interaction picture with respect to the free electron Hamiltonian $H_{0}^{e}$. The dynamics of the free electronic field operators is then determined by the relations

$$
\begin{aligned}
& \psi_{e r ; 0}(x, t)=\psi_{e r}\left(x-v_{f} t\right) e^{i v_{f} k_{f} t} \\
& \psi_{e l ; 0}(x, t)=\psi_{e l}\left(x+v_{f} t\right) e^{i v_{f} k_{f} t} .
\end{aligned}
$$

These results can also be obtained without applying the bosonization technique. The free time behavior of the generators for the unitary transformation $\mathcal{S}$ is described by the equations

$$
\chi_{r ; 0}(y, t)=\chi_{r}\left(y-v_{f} t\right)=-i \sum_{q>0} \sqrt{\frac{2 \pi}{L q}} \frac{\tilde{v}(q)}{2 \pi v_{f}} e^{i q\left(y-v_{f} t\right)} b_{q r}
$$


and

$$
\chi_{l ; 0}(y, t)=\chi_{l}\left(y+v_{f} t\right)=-i \sum_{q>0} \sqrt{\frac{2 \pi}{L q}} \frac{\tilde{v}(q)}{2 \pi v_{f}} e^{-i q\left(y+v_{f} t\right)} b_{q l}
$$

which show a similar structure as the corresponding equations fore $\varphi_{r ; 0}$ and $\varphi_{l ; 0}$.

In order to calculate the response functions, it is necessary to evaluate the different kinds of commutator for the bosonic field operators. If the particles do not interact with each other, only the commutators

$$
\begin{aligned}
{\left[\varphi_{r ; 0}(x, t), \varphi_{r ; 0}^{\dagger}\left(x^{\prime}, t^{\prime}\right)\right] } & =\lim _{\alpha \searrow 0} \sum_{q>0} \frac{2 \pi}{L q} e^{-2 \alpha q} e^{i q\left\{\left(x-x^{\prime}\right)-v_{f}\left(t-t^{\prime}\right)\right\}} \\
& =-\lim _{\alpha \searrow 0} \ln \left(1-e^{-\left\{2 \alpha-i\left(x-x^{\prime}\right)+i v_{f}\left(t-t^{\prime}\right)\right\} \frac{2 \pi}{L}}\right)
\end{aligned}
$$

and

$$
\begin{aligned}
{\left[\varphi_{l ; 0}(x, t), \varphi_{l ; 0}^{\dagger}\left(x^{\prime}, t^{\prime}\right)\right] } & =\lim _{\alpha \searrow 0} \sum_{q>0} \frac{2 \pi}{L q} e^{-2 \alpha q} e^{-i q\left\{\left(x-x^{\prime}\right)+v_{f}\left(t-t^{\prime}\right)\right\}} \\
& =-\lim _{\alpha \searrow 0} \ln \left(1-e^{-\left\{2 \alpha+i\left(x-x^{\prime}\right)+i v_{f}\left(t-t^{\prime}\right)\right\} \frac{2 \pi}{L}}\right)
\end{aligned}
$$

give a non-vanishing contribution. In the presence of the electron-hole interaction, however, the following two additional types of commutators

$$
\begin{aligned}
& {\left[\chi_{r ; 0}(y, t), \chi_{r ; 0}^{\dagger}\left(y_{1}, t_{1}\right)-\chi_{r ; 0}^{\dagger}\left(y_{2}, t_{2}\right)\right]=H\left(y-y_{1}-v_{f}\left(t-t_{1}\right), y-y_{2}-v_{f}\left(t-t_{2}\right)\right),} \\
& {\left[\varphi_{r ; 0}(x, t), \chi_{r ; 0}^{\dagger}\left(y_{1}, t_{1}\right)-\chi_{r ; 0}^{\dagger}\left(y_{2}, t_{2}\right)\right]=G\left(x-y_{1}-v_{f}\left(t-t_{1}\right), x-y_{2}-v_{f}\left(t-t_{2}\right)\right)}
\end{aligned}
$$

and

$$
\begin{aligned}
& {\left[\chi_{l ; 0}(y, t), \chi_{l ; 0}^{\dagger}\left(y_{1}, t_{1}\right)-\chi_{l ; 0}^{\dagger}\left(y_{2}, t_{2}\right)\right]=H^{*}\left(y-y_{1}+v_{f}\left(t-t_{1}\right), y-y_{2}+v_{f}\left(t-t_{2}\right)\right),} \\
& {\left[\varphi_{l ; 0}(x, t), \chi_{l ; 0}^{\dagger}\left(y_{1}, t_{1}\right)-\chi_{l ; 0}^{\dagger}\left(y_{2}, t_{2}\right)\right]=G^{*}\left(x-y_{1}+v_{f}\left(t-t_{1}\right), x-y_{2}+v_{f}\left(t-t_{2}\right)\right)}
\end{aligned}
$$

have to be taken into account. The auxiliary functions $G$ and $H$ are defined by the following equations

$$
G\left(x, x^{\prime}\right)=\sum_{q>0} \frac{2 \pi}{L q} \frac{\tilde{v}(q)}{2 \pi v_{f}}\left\{e^{i q x}-e^{i q x^{\prime}}\right\} \underset{L \rightarrow \infty}{=} \int_{0}^{\infty} \frac{d q}{q} \frac{\tilde{v}(q)}{2 \pi v_{f}}\left\{e^{i q x}-e^{i q x^{\prime}}\right\}
$$


and

$$
H\left(x, x^{\prime}\right)=\sum_{q>0} \frac{2 \pi}{L q} \frac{\tilde{v}^{2}(q)}{\left(2 \pi v_{f}\right)^{2}}\left\{e^{i q x}-e^{i q x^{\prime}}\right\} \underset{L \rightarrow \infty}{=} \int_{0}^{\infty} \frac{d q}{q} \frac{\tilde{v}^{2}(q)}{\left(2 \pi v_{f}\right)^{2}}\left\{e^{i q x}-e^{i q x^{\prime}}\right\} .
$$

Unfortunately, explicit solutions for the two integrals in Equations D.15 and D.16 exist only for a limited number of interaction functions $\tilde{v}(q)$. Nevertheless, it is possible to determine the leading behavior of these functions for large values of $x$ and $x^{\prime}$. This is due to the existence of the two general relations

$$
\int_{0}^{\infty} d q f(q) \frac{\sin (q x)}{q}=\frac{\pi}{2} f(0)+\frac{1}{x} f^{\prime}(0)-\frac{1}{x^{2}} R_{f}^{s}(x)
$$

with

$$
R_{f}^{s}(x)=\int_{0}^{\infty} d q f^{\prime \prime \prime}(q)\left\{\frac{(q x)^{2}}{2} \operatorname{si}(q x)+\frac{q x}{2} \cos (q x)+\frac{1}{2} \sin (q x)\right\}
$$

and

$$
\int_{0}^{\infty} d q f(q) \frac{\cos (q x)-1}{q}=-f(0) \ln (x)+\int_{0}^{\infty} d q f^{\prime}(q)\left\{\ln (q)+C_{e}\right\}-\frac{1}{x^{2}} R_{f}^{c}(x)
$$

with

$$
R_{f}^{c}(x)=\int_{0}^{\infty} d q f^{\prime \prime \prime}(q)\left\{\frac{(q x)^{2}}{2} \operatorname{ci}(q x)-\frac{q x}{2} \sin (q x)+\frac{1}{2} \cos (q x)-\frac{1}{2}\right\}
$$

which are valid for positive $x . C_{e}=0.5772 \ldots$ is the Euler constant and the functions si and ci represent the sine and the cosine integral respectively [7]. The function $f$ is integrable and can at least be differentiated three times where the derivatives up to the third order are also integrable. These requirements are necessary since the integrals on the left-hand sides of Equations D.17 and D.19 have to be integrated partially three times in order to obtain the expressions on the right-hand sides. The absolute values of the expressions in the curved brackets which appear in Equations D.18 and D.20 can be estimated by means of a common boundary constant $K_{R}>0$ which is independent of $x$. Consequently, the integrals in Equation D.17 and Equation D.19 are approximately described by the relations

$$
\int_{0}^{\infty} d q f(q) \frac{\sin (q x)}{q} \approx \frac{\pi}{2} f(0)
$$

and

$$
\int_{0}^{\infty} d q f(q) \frac{\cos (q x)-1}{q} \approx-f(0) \ln (x)+\int_{0}^{\infty} d q f^{\prime}(q)\left\{\ln (q)+C_{e}\right\}
$$


provided that

$$
x \gg x_{f}=\max \left(\sqrt{K_{R}} \sqrt{\int_{0}^{\infty} d q\left|f^{\prime \prime \prime}(q)\right|},\left|f^{\prime}(0)\right|\right) .
$$

The application of the above formulae to the auxiliary functions in Equations D.15 and D.16 yields the following approximations

$$
G(x, 0) \approx-\frac{\tilde{v}(0)}{2 \pi v_{f}} \ln (x)+c_{G}+i \frac{\tilde{v}(0)}{2 \pi v_{f}} \frac{\pi}{2}
$$

and

$$
H(x, 0) \approx-\frac{\tilde{v}^{2}(0)}{\left(2 \pi v_{f}\right)^{2}} \ln (x)+c_{H}+i \frac{\tilde{v}^{2}(0)}{\left(2 \pi v_{f}\right)^{2}} \frac{\pi}{2}
$$

for $x \gg x_{\tilde{v}}=\max \left(x_{G}, x_{H}\right)$ where the constants $c_{G}$ and $c_{H}$ are defined by the equations

$$
c_{G}=\int_{0}^{\infty} d q\left\{\ln (q)+C_{e}\right\} \frac{d}{d q} \frac{\tilde{v}(q)}{2 \pi v_{f}}
$$

and

$$
c_{H}=\int_{0}^{\infty} d q\left\{\ln (q)+C_{e}\right\} \frac{d}{d q} \frac{\tilde{v}^{2}(q)}{\left(2 \pi v_{f}\right)^{2}}
$$

while the constants $x_{G}$ and $x_{H}$ satisfy the relations

$$
x_{G}=\max \left(\sqrt{K_{R}} \sqrt{\int_{0}^{\infty} d q\left|\frac{d^{3}}{d q^{3}} \frac{\tilde{v}(q)}{2 \pi v_{f}}\right|},\left|\frac{d}{d q} \frac{\tilde{v}(q)}{2 \pi v_{f}}\right|_{q=0}\right)
$$

and

$$
x_{H}=\max \left(\sqrt{K_{R}} \sqrt{\int_{0}^{\infty} d q\left|\frac{d^{3}}{d q^{3}} \frac{\tilde{v}^{2}(q)}{\left(2 \pi v_{f}\right)^{2}}\right|},\left|\frac{d}{d q} \frac{\tilde{v}^{2}(q)}{\left(2 \pi v_{f}\right)^{2}}\right|_{q=0}\right) .
$$

The Equations D.17 and D.19 can also be employed to derive the two general relations

$$
H\left(y-y_{1}, y-y_{2}\right) \approx-\frac{\tilde{v}^{2}(0)}{\left(2 \pi v_{f}\right)^{2}} \ln \left(\frac{\left|y-y_{1}\right|}{\left|y-y_{2}\right|}\right)+i \frac{\tilde{v}^{2}(0)}{\left(2 \pi v_{f}\right)^{2}} \pi\left\{\theta\left(y-y_{1}\right)-\theta\left(y-y_{2}\right)\right\}
$$

and

$$
G\left(x-x_{1}, x-x_{2}\right) \approx-\frac{\tilde{v}(0)}{2 \pi v_{f}} \ln \left(\frac{\left|x-x_{1}\right|}{\left|x-x_{2}\right|}\right)+i \frac{\tilde{v}(0)}{2 \pi v_{f}} \pi\left\{\theta\left(x-x_{1}\right)-\theta\left(x-x_{2}\right)\right\}
$$




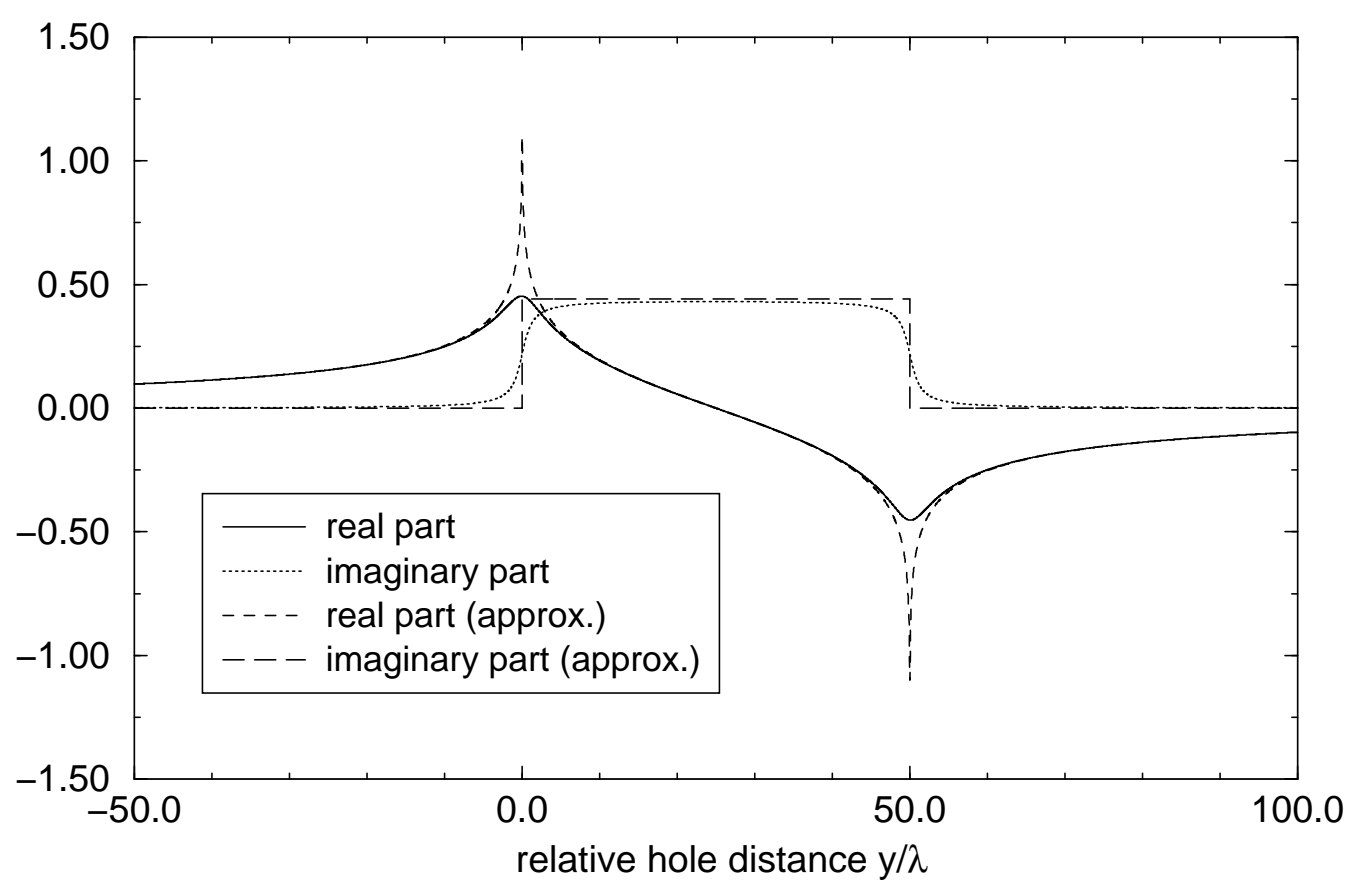

Figure D.1: The function $H\left(y-y_{1}, y-y_{2}\right)$ with $\frac{\tilde{v}_{0}}{2 \pi v_{f}}=-0.375, y_{1}=0.0$ and $y_{2}=50 \lambda$

which are valid, if $\left|y-y_{1}\right|,\left|y-y_{2}\right| \gg x_{\tilde{v}}$ and $\left|x-x_{1}\right|,\left|x-x_{2}\right| \gg x_{\tilde{v}}$. It turns out that these approximations are very useful for the calculation of the four-point response functions in Appendix C. The same is true for the relations

$$
\begin{aligned}
H\left(y-y_{1},-y+y_{2}\right) & \approx-\frac{\tilde{v}^{2}(0)}{\left(2 \pi v_{f}\right)^{2}} \ln \left(\frac{\left|y-y_{1}\right|}{\left|y-y_{2}\right|}\right) \\
& +i \frac{\tilde{v}^{2}(0)}{\left(2 \pi v_{f}\right)^{2}} \pi\left\{\theta\left(y-y_{1}\right)+\theta\left(y-y_{2}\right)-1\right\}
\end{aligned}
$$

and

$$
\begin{aligned}
G\left(x-x_{1},-x+x_{2}\right) & \approx-\frac{\tilde{v}(0)}{2 \pi v_{f}} \ln \left(\frac{\left|x-x_{1}\right|}{\left|x-x_{2}\right|}\right) \\
& +i \frac{\tilde{v}(0)}{2 \pi v_{f}} \pi\left\{\theta\left(x-x_{1}\right)+\theta\left(x-x_{2}\right)-1\right\}
\end{aligned}
$$

which can also be used, if $\left|y-y_{1}\right|,\left|y-y_{2}\right| \gg x_{\tilde{v}}$ and $\left|x-x_{1}\right|,\left|x-x_{2}\right| \gg x_{\tilde{v}}$.

If the Fourier transform of the interaction potential is given by $\tilde{v}(q)=\tilde{v}_{0} e^{-\lambda|q|}$ with $\tilde{v}_{0} \in \mathbb{R}$ and $\lambda \in \mathbb{R}^{+}$, the integrals can be performed exactly [7] and the following equations

$$
G\left(x, x^{\prime}\right)=-\frac{\tilde{v}_{0}}{2 \pi v_{f}} \ln \sqrt{\frac{x^{2}+\lambda^{2}}{x^{\prime 2}+\lambda^{2}}}+i \frac{\tilde{v}_{0}}{2 \pi v_{f}}\left\{\arctan \left(\frac{x}{\lambda}\right)-\arctan \left(\frac{x^{\prime}}{\lambda}\right)\right\}
$$




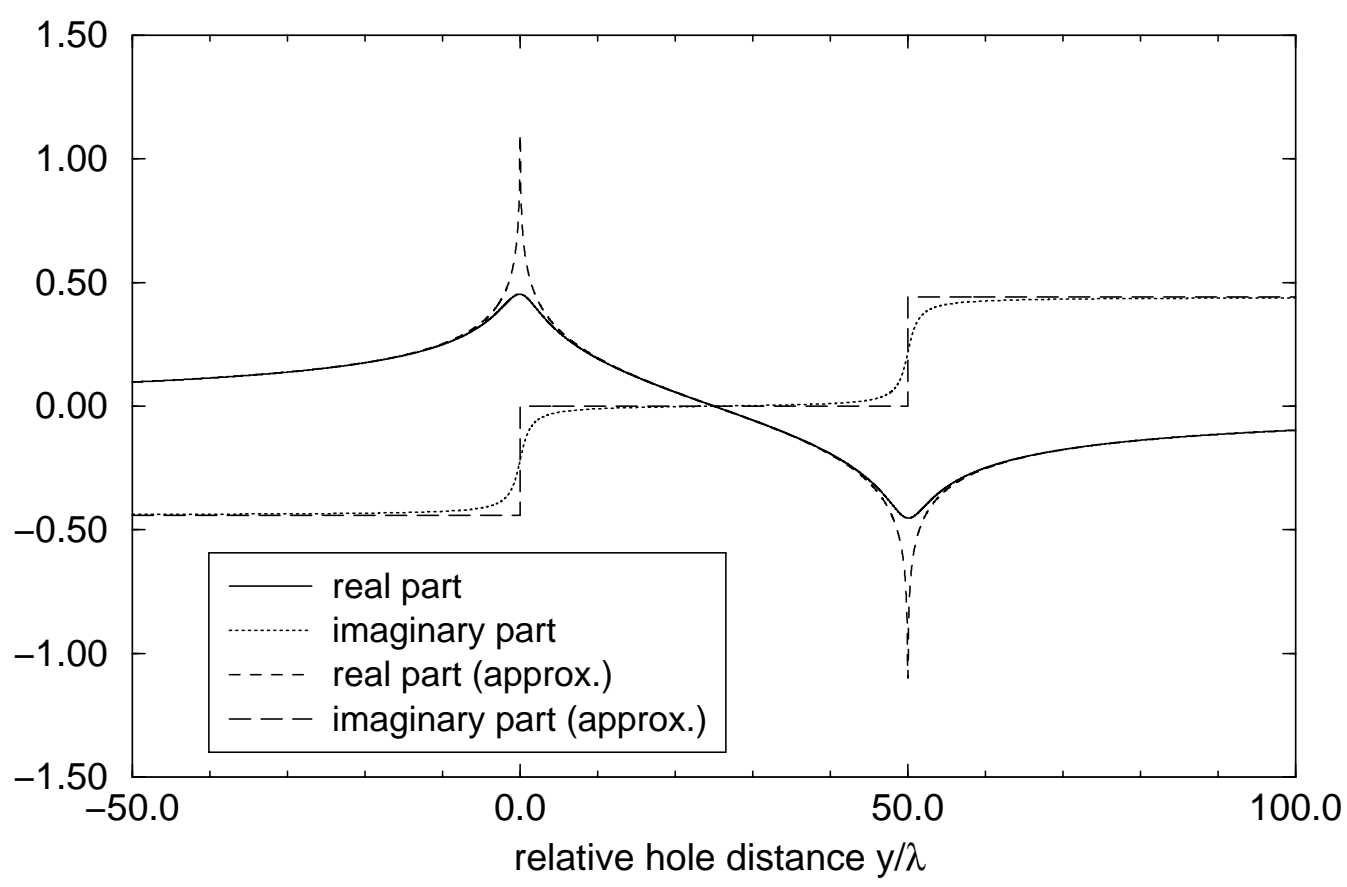

Figure D.2: The function $H\left(y-y_{1},-y+y_{2}\right)$ with $\frac{\tilde{v}_{0}}{2 \pi v_{f}}=-0.375, y_{1}=0.0$ and $y_{2}=50 \lambda$

and

$$
H\left(x, x^{\prime}\right)=-\frac{\tilde{v}_{0}^{2}}{\left(2 \pi v_{f}\right)^{2}} \ln \sqrt{\frac{x^{2}+4 \lambda^{2}}{x^{\prime 2}+4 \lambda^{2}}}+i \frac{\tilde{v}_{0}^{2}}{\left(2 \pi v_{f}\right)^{2}}\left\{\arctan \left(\frac{x}{2 \lambda}\right)-\arctan \left(\frac{x^{\prime}}{2 \lambda}\right)\right\}
$$

hold. With the help of the exact expressions for $G$ and $H$, it is now possible to estimate the quality of the approximated expressions on the right-hand sides of Equations D.30 to D.33. In order to give an example, the real and the imaginary parts of the functions $H\left(y-y_{1}, y-y_{2}\right)$ and $H\left(y-y_{1},-y+y_{2}\right)$ have been plotted together with the corresponding approximated curves for a specific choice of $\lambda$ and $\tilde{v}_{0}$ in Figures D. 1 and D.2. As expected, the approximated curves differ from the exact ones only in the vicinities of the points $y=y_{1}$ and $y=y_{2}$. The extension of the regions where the differences between the exact and the approximated expressions for $G$ and $H$ cannot be neglected is estimated with the help of the constant

$$
x_{\tilde{v}}=\max \left(x_{G}, x_{H}\right)=\lambda \max \left(\sqrt{K_{R}} \sqrt{\left|\frac{\tilde{v}_{0}}{2 \pi v_{f}}\right|},\left|\frac{\tilde{v}_{0}}{2 \pi v_{f}}\right|, \sqrt{K_{R}}\left|\frac{\tilde{v}_{0}}{2 \pi v_{f}}\right|,\left|\frac{\tilde{v}_{0}}{2 \pi v_{f}}\right|^{2}\right) .
$$




\section{Appendix E}

\section{Important Functions}

The following list shows where the definitions for important auxiliary functions can be found:

$$
\begin{array}{lc}
G\left(x, x^{\prime}\right) & \text { in Equation D.15, } \\
H\left(x, x^{\prime}\right) & \text { in Equation D.16, } \\
s_{X ; r}\left(t_{1}, t_{2}, x\right) & \text { in Equation 3.53, } \\
s_{X ; l}\left(t_{1}, t_{2}, x\right) & \text { in Equation 3.54, } \\
S_{X ; r r}\left(t_{1}, t_{2}, t_{3}, t_{4} ; y^{\prime} ; x_{1}, x_{2}, y\right) & \text { in Equation C.8, } \\
S_{X ; r l}\left(t_{1}, t_{2}, t_{3}, t_{4} ; y^{\prime} ; x_{1}, x_{2}, y\right) & \text { in Equation C.11, } \\
S_{X ; l r}\left(t_{1}, t_{2}, t_{3}, t_{4} ; y^{\prime} ; x_{1}, x_{2}, y\right) & \text { in Equation C.12, } \\
S_{X X ; r r}\left(t_{1}, t_{2}, t_{3}, t_{4} ; y^{\prime} ; x_{1}, x_{2}, y\right) & \text { in Equation C.14, } \\
S_{X X ; r l}\left(t_{1}, t_{2}, t_{3}, t_{4} ; y^{\prime} ; x_{1}, x_{2}, y\right) & \text { in Equation C.17, } \\
S_{X X ; l r}\left(t_{1}, t_{2}, t_{3}, t_{4} ; y^{\prime} ; x_{1}, x_{2}, y\right) & \text { in Equation C.18. }
\end{array}
$$

The following list shows where the definitions for important response functions and the corresponding approximated expressions can be found:

$$
\begin{array}{ll}
\left\langle\left\langle\tilde{P}_{r}\left(t_{1}\right) \tilde{P}_{r}^{\dagger}\left(t_{2}\right)\right\rangle\right\rangle & \text { in Equations 3.55 and 3.56, } \\
\left\langle\left\langle\tilde{P}_{r}\left(t_{1}\right) \tilde{P}_{r}^{\dagger}\left(t_{2}\right) \tilde{P}_{r}\left(t_{3}\right) \tilde{P}_{r}^{\dagger}\left(t_{4}\right)\right\rangle\right\rangle_{c} & \text { in Equations C.19 and C.38, } \\
\left\langle\left\langle\tilde{P}_{r}\left(t_{1}\right) \tilde{P}_{r}^{\dagger}\left(t_{2}\right) \tilde{P}_{l}\left(t_{3}\right) \tilde{P}_{l}^{\dagger}\left(t_{4}\right)\right\rangle\right\rangle_{c} & \text { in Equations C.23 and C.39, } \\
\left\langle\left\langle\tilde{P}_{r}\left(t_{1}\right) \tilde{P}_{l}^{\dagger}\left(t_{2}\right) \tilde{P}_{l}\left(t_{3}\right) \tilde{P}_{r}^{\dagger}\left(t_{4}\right)\right\rangle\right\rangle_{c} & \text { in Equations C.24 and C.40, } \\
\left\langle\left\langle\tilde{P}_{r}\left(t_{1}\right) \tilde{P}_{r}\left(t_{2}\right) \tilde{P}_{r}^{\dagger}\left(t_{3}\right) \tilde{P}_{r}^{\dagger}\left(t_{4}\right)\right\rangle\right\rangle_{c} & \text { in Equations C.27 and C.41, } \\
\left\langle\left\langle\tilde{P}_{r}\left(t_{1}\right) \tilde{P}_{l}\left(t_{2}\right) \tilde{P}_{r}^{\dagger}\left(t_{3}\right) \tilde{P}_{l}^{\dagger}\left(t_{4}\right)\right\rangle\right\rangle_{c} & \text { in Equations C.32 and C.42, } \\
\left\langle\left\langle\tilde{P}_{r}\left(t_{1}\right) \tilde{P}_{l}\left(t_{2}\right) \tilde{P}_{l}^{\dagger}\left(t_{3}\right) \tilde{P}_{r}^{\dagger}\left(t_{4}\right)\right\rangle\right\rangle_{c} & \text { in Equations C.33 and C.43. }
\end{array}
$$




\section{Bibliography}

[1] S. Tomonaga, Prog. Theo. Phys. 5, 544 (1950)

[2] T. Holstein, Ann. Phys. (New York) 8, 325 (1959)

[3] T. Holstein, Ann. Phys. (New York) 8, 343 (1959)

[4] P. C. Martin and J. S. Schwinger, Phys. Rev. 115, 1342 (1959)

[5] L. P. Kadanoff and G. Baym, Quantum Statistical Mechanics (Benjamin, 1962)

[6] J. M. Luttinger, J. Math. Phys. 4, 1154 (1963)

[7] I. S. Gradshteyn and I. M. Ryzhik, Table of Integrals, Series and Products (Academic Press, 1965)

[8] L. V. Keldysh, Sov. Phys. JETP 20, 1018 (1965)

[9] P. W. Anderson, Phys. Rev. Lett. 18, 1049 (1967)

[10] G. D. Mahan, Phys. Rev. 153, 882 (1967)

[11] J. Gavoret, P. Nozières, B. Roulet and M. Combescot, J. Phys. (Paris) 30, 987 (1969)

[12] B. Roulet, J. Gavoret and P. Nozières, Phys. Rev. 178, 1072 (1969)

[13] P. Nozières, J. Gavoret and B. Roulet, Phys. Rev. 178, 1084 (1969)

[14] P. Nozières and C. T. De Dominicis, Phys. Rev. 178, 1097 (1969)

[15] K. D. Schotte and U. Schotte, Phys. Rev. 182, 479 (1969)

[16] M. Combescot and P. Nozières, J. Phys. (Paris) 32, 913 (1971)

[17] F. D. M. Haldane, J. Phys. C 14, 2585 (1981)

[18] P. Lipavský, V. Špička and B. Velický, Phys. Rev. B 34, 6933 (1986)

[19] G. D. Mahan, Many-Particle Physics (Plenum Press, 1990) 
[20] T. Uenoyama and L. J. Sham, Phys. Rev. Lett. 65, 1048 (1990)

[21] R. Zimmermann, Phys. Status Solidi B 159, 317 (1990)

[22] J. M. Calleja, A. R. Goñi, B. S. Dennis, J. S. Weiner, A. Pinczuk, S. SchmittRink, L. N. Pfeiffer, K. W. West, J. F. Müller and A. E. Ruckenstein, Solid State Commun. 79, 911 (1991)

[23] I. E. Perakis and Y.-C. Chang, Phys. Rev. B 43, 12556 (1991)

[24] I. E. Perakis and Y.-C. Chang, Phys. Rev. B 44, 5877 (1991)

[25] L. Bányai, D. B. Tran Thoai, C. Remling and H. Haug, Phys. Status Solidi B 173, 149 (1992)

[26] H. Haug, Phys. Status Solidi B 173, 139 (1992)

[27] D. K. K. Lee and Y. Chen, Phys. Rev. Lett. 69, 1399 (1992)

[28] J. F. Mueller, A. E. Ruckenstein and S. Schmitt-Rink, Phys. Rev. B 45, 8902 (1992)

[29] T. Ogawa, A. Furusaki and N. Nagaosa, Phys. Rev. Lett. 68, 3638 (1992)

[30] R. Zimmermann, J. Lumin. 53, 187 (1992)

[31] H. Haug and S. W. Koch, Quantum Theory of the Optical and Electronic Properties of Semiconductors (World Scientific, 1993)

[32] Th. Östreich, doctoral thesis, Göttingen (1993)

[33] I. E. Perakis and Y.-C. Chang, Phys. Rev. B 47, 6573 (1993)

[34] D. B. Tran Thoai and H. Haug, Phys. Rev. B 47, 3574 (1993)

[35] I. E. Perakis and D. S. Chemla, Phys. Rev. Lett. 72, 3202 (1994)

[36] J. Schilp, T. Kuhn and G. Mahler, Phys. Rev. B 50, 5435 (1994)

[37] H. Schoeller, Ann. Phys. (New York) 229, 273 (1994)

[38] R. Zimmermann and J. Wauer, J. Lumin. 58, 271 (1994)

[39] L. Bányai, D. B. Tran Thoai, E. Reitsamer, H. Haug, D. Steinbach, M. U. Wehner, M. Wegener, T. Marschner and W. Stolz, Phys. Rev. Lett. 75, 2188 (1995)

[40] V. Meden, C. Wöhler, J. Fricke and K. Schönhammer, Phys. Rev. B 52, 5624 (1995)

[41] V. M. Axt, K. Victor and A. Stahl, Phys. Rev. B 53, 7244 (1996) 
[42] J. Fricke, Ann. Phys. (New York) 252, 479 (1996)

[43] H. Haug and A.-P. Jauho, Quantum Kinetics in Transport and Optics of Semiconductors (Springer, 1996)

[44] I. E. Perakis, I. Brener, W. H. Knox and D. S. Chemla, J. Opt. Soc. Am. B 13, 1313 (1996)

[45] Y. Oreg and A. M. Finkel'stein, Phys. Rev. B 53, 10928 (1996)

[46] H. Otani and T. Ogawa, Phys. Rev. B 54, 4540 (1996)

[47] C. Fürst, A. Leitenstorfer, A. Laubereau and R. Zimmermann, Phys. Rev. Lett. 78, 3733 (1997)

[48] K. Schönhammer and C. Wöhler, Phys. Rev. B 55, 13564 (1997)

[49] Th. Östreich, Phys. Status Solidi A 164, 313 (1997)

[50] V. Meden, P. Schmitteckert and N. Shannon, Phys. Rev. B 57, 8878 (1998)

[51] Th. Östreich, K. Schönhammer and L. J. Sham, Phys. Rev. B 58, 12920 (1998)

[52] Th. Östreich, N. Donlagic, C. Wöhler and K. Schönhammer, Phys. Status Solidi B 206, 205 (1998)

[53] M. U. Wehner, M. H. Ulm, D. S. Chemla and M. Wegener, Phys. Rev. Lett. 80, $1992(1998)$

[54] C. Wöhler, doctoral thesis, Göttingen (1998)

[55] R. Zimmermann, J. Wauer, A. Leitenstorfer and C. Fürst, J. Lumin. 76-77, 34 (1998)

[56] N. Donlagic and Th. Östreich, Phys. Rev. B 59, 7493 (1999)

[57] J. Shah, Ultrafast Spectroscopy of Semiconductors and Semiconductor Nanostructures (Springer, 1999)

[58] K. Hannewald, S. Glutsch and F. Bechstedt, Phys. Rev. B 61, 10792 (2000)

[59] T. V. Shahbazyan, N. Primozich, I. E. Perakis and D. S. Chemla, Phys. Rev. Lett. 84, 2006 (2000)

[60] D. Porras, J. Fernández-Rossier and C. Tejedor, cond-mat/0009156 (2000) 



\section{Acknowledgements}

In the first place I would like to thank Prof. Dr. Kurt Schönhammer for giving me the opportunity to write this thesis and for his readiness to discuss the results with me. After having introduced me to the field of theoretical physics as a teacher he supported my scientific work with useful suggestions and comments.

I am greatly indebted to Dr. Thomas Östreich for his moral encouragement and his helpfulness during the years of our collaboration which I enjoyed very much. Furthermore, I thank him for critically reading the manuscript of my thesis.

In addition, I would especially like to thank Prof. Lu Sham who drew my attention to the interesting features of condensed matter systems with a Fermi-edge singularity initiating the studies whose results are summarized in Chapter 3 of my doctoral thesis.

During my stay at the Lawrence Berkeley National Laboratory I had the pleasure to enjoy the kind hospitality of Prof. Daniel Chemla and his group. I would like to thank all of them for giving me an insight into the world of experimental physicists.

I also want to thank Dr. Jürgen Holm for the patience he showed when talking with me about my programming problems. His advice in all questions concerning computers has always been very helpful for me.

While writing my doctoral thesis, I learned to appreciate the friendly and encouraging atmosphere in our group for which I would like to thank all present and former group members. I am particularly indebted to Dr. Lorenz Bartosch who provided essential assistance in the last stage of my work.

My special thanks go to my former roommate Dr. Thorsten Köhler who shared the office with me for four years. He has always been open for physical and nonphysical discussions from which I benefitted a lot.

Finally, I would like to express my deepest thanks to my family. Their grateful support and their confidence accompanied me during my entire studies. 



\title{
Lebenslauf
}

\author{
Nachname: $\quad$ Đonlagić \\ Vornamen: Nias Sven \\ Geburtstag: 19. Juli 1971 \\ Geburtsort: Lübeck \\ Familienstand: ledig, keine Kinder \\ Staatsangehörigkeit: deutsch \\ $1978-1991$ \\ 8. Juni 1991 \\ Grundschule und Gymnasium in Lübeck \\ Abitur
}

Oktober 1991

Immatrikulation für den Diplomstudiengang Physik

an der Georg-August-Universität in Göttingen

15. Oktober 1993

Vordiplom (Physik)

März 1995 - Okt. 1996

Anfertigung einer Diplomarbeit im Institut für Theoretische Physik

der Georg-August-Universität zu Göttingen

Titel der Diplomarbeit:

Relaxation in niedrigdimensionalen korrelierten

Fermisystemen

Betreuer:

Prof. Dr. Kurt Schönhammer

6. Februar 1997

Diplom (Physik)

Seit März 1997

Wissenschaftlicher Mitarbeiter und Doktorand

im Institut für Theoretische Physik

der Georg-August-Universität zu Göttingen

Titel der Doktorarbeit:

Nonlinear Optical Effects in Pure and N-Doped

Semiconductors

Betreuer:

Prof. Dr. Kurt Schönhammer 